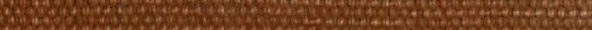




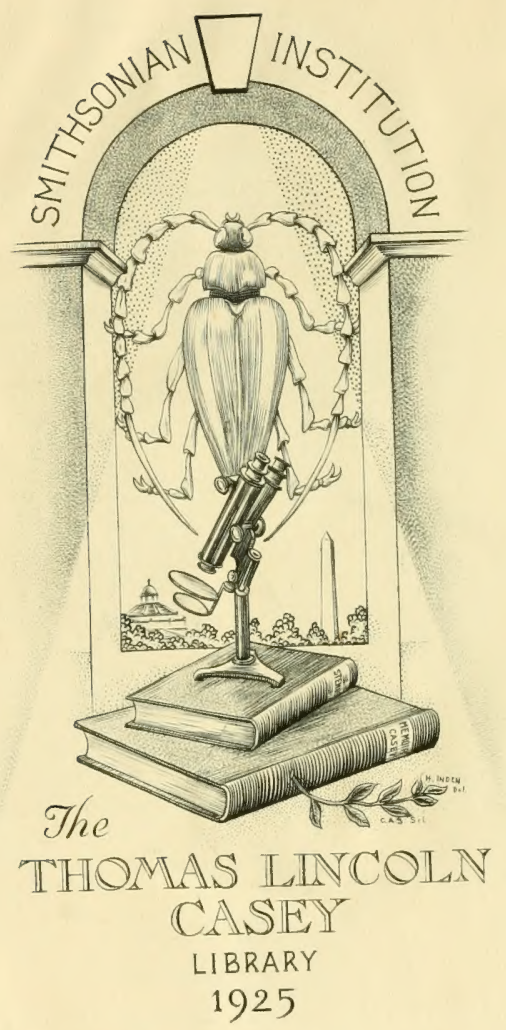



Mannorherm, Cav1 Gurtav, count, 1949.18 Kaefer -7 an nh. 


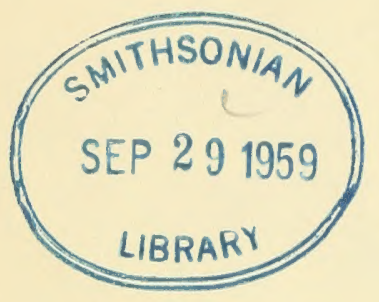




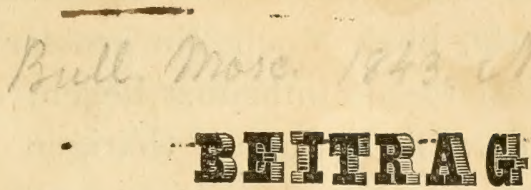

ŹUR

\section{RAEFET-FAUNA}

DER ALEUTISCHEN INSELN, DER INSEL SITKHA

UN D

\section{NEU-GALIFORNIENS}

\author{
von Graf C. G. Mannerheim.
}

Obgleich der Herr Professor Doctor Johann Friedrich Eschscholtz erst in seinen Entomographien $\left({ }^{*}\right)$ und hernach in dem von ihm herausgegebenen zoologischem Atlas zur zweiten Reise des Herrn Flott-Capitains Otto von Kotzebue ( $\left.{ }^{* *}\right)$ eine Menge, während der beiden mit genanntem Herrn Capitain zurückgelegten Reisen um die

$\left({ }^{*}\right)$ Entomographien von J. Friedrich Eschscholtz. Erste Lieferung. Berlin, 1842, in $8^{\circ}$.

$\left({ }^{* *}\right)$ Zoologischer Atlas, enthaltend Abbildangen und Beschreibungen neuer Thiørarten, während der Flott-Capitains von Kotzebue zweiter Reise- um diēWelt, beobachtet von $D^{\text {r. }}$ Friedrich Eschscoltz_Fün. Hefte in Folio Drlin, 1829-1833. No I. 1843. 
Welt, gemachten entomologischen Entdeckungen veröffentlicht hatte, blieb doch ein grosser Theil des gesammelten Materials unbenuzt liegen, da am $7^{\text {ten }}$ Mai 1831 der Tod diesen thätiggen Mann dahinraffte und so der Naturgeschichte einen der gründlichsten und ausgezeichnetesten Entomologen unseres Zeitalters entriss. Die persönliche Bekanntschaft, die ich mit dem Herrn Doctor Eschscholtz, schon vor seiner zweiten Reise um die Welt, anknüpfte und die seine freundschaftlichen Gesinnungen gegen mich mit jedem Tage vermehrte, von denen ich die angenehmsten Eriunerungen durch das ganze Leben behalten werde, gab die Veranlassung zu einem fleissigen Briefwechsel zwischen uns. Auch während der Weltumschiffung von 1823 bis 1826 theilte er mir in Briefen aus Sitkha und Californien die von ihm auf der Reise gemachten überaus interessanten entomologischen Beobachtungen mit, und heimgekehrt bereicherte er vorzugsweise meine Sammlung mit seinen vielen neuen Entdeckungen, so dass mein entomologisches $\mathrm{K}$ abinet eines der vollständigsten in Hinsicht derselben werden musste. Seitdem unterhielt er mit mir über diese Gegenstände eine äusserst belehrende Correspondenz und, oftmals war ich gesonnen die daraus gezogenen Aufklärungen über die entomologischen Schätze dieser entlegenen Gegenden im Druck bekannt zu machen. Allein es kamen mir manche Hindernisse in den Weg, die diese Absicht vereitelien. Lange waren unsere 
Kenntnisse der Insecten dieser von dem stillen Oceane bewässerten, in der nördlichen Hemisphäre liegenden Küsten- und Insel-Länder nur auf die von Eschscholtz daselbst eingesammelten Arten beschränkt, bis im Herbste 1841 der aus Sithka heimgekehrte Doctor Blaschke eine Sammlung von genannter Insel und von Californien mitbrachte, wovon eine schöne Reihenfolgge der interessantesten Käfer durch die, bei mir stets in der dankbarsten Erinnerung bewahrte, besondere Güte und Freundschaft $\mathrm{S}^{\mathrm{r}}$ Excellenz des Herrn wirklichen Staats-Raths Doctor Lange mir zu 'Theil wurde.-Fast zu gleicher Zeit hatte der gewesene Gouverneur der Besitzungen der russisch-amerikanischen Compagnie, Herrn Flott-Capitain ersten Ranges Kuprianoff, die ausgezeichnete Gefälligkeit mir eine Menge der von ihm aus Sitkha mitgebrachten Insecten zu verehren und es gereicht mir zur angenehmsten Pflicht, diesem für die Kenntniss der Naturerzeugnisse der seiner Verwaltung anvertrauten Länder so thätigen Manne meine ganz besondere Erkenntlichkeit für diese mir erzeigte Güte hiemit öffentlich abzustatten. Bevor noch diese jezt erwähnten Sendungen bei mir eingingen, erwies der Herr FlottCapitain-Lieutenant Behrens mir die Freundschaft einige von seinem Reisegefährțen nach Sitkha und Californien, dem Herrn Doctor Fischer, in der vormaligen russischen Colonie Ross eingesammelten und in Weingeist aufbewahrten Käfer zu 
schicken, von welchen ich zwei ausgezeichnete Arten in der Revue zoologique par la Société Cuvierienne, année 1840. S. 137. schon beschrieben habe.

Mit diesen zusammengebrachten Materialien ausgestattet beabsichtigte ich eine Käfer-Fauna der Aleutischen Inseln, der Insel Sitkha und der mit der russisch-amerikanischen Compagnie in Beziehung gestandenen 'Theile Neu-Californiens auszuarbeiten und zugleich in dieses Werk alles zusammenzubringen, was in verschiedenen entomologischen Schriften über die Käfer dieser Gegenden bisher geliefert worden ist. Mit diesem Entschlusse wandte ich mich an Seine Excellenz, den Herrn wirklichen Staats-Rath Fischer von Waldheim, Vice-President der Kayserl. naturforschenden Gesellschaft zu Moskau und an Herrn Ménétriés, Conservator des entomologischen Museums der Kayserl. Akademie der Wissenschaften zu St. Petersburg, um die in den unter ihrer Aufsicht stehenden Sammlungen etwa vorhandenen Käfer der gedachten Länder in die erwähnte Fauna aufnehmen zu können, was mir um so mehr zum Bedürfnisse gereichte, da das gewesene entomologische Kabinet des verstorbenen Eschscholtz durch Kauf ein Eigenthum der Moskauer Universität geworden war. Die freundschaftliche Gefälligkeit, womit diese Herren meine Wünsche hierin befriedigten, ist über alles Lob erhaben und die Bciträge, die ich dadurch erhielt, gehören ge- 
wiss zu den interessantesten bei der Vervollständigung meines beabsichtigten Werkes; insbesondere waren einige von Herrn Ménétriés mitgetheilte Käfer ausgezeichnet, die der vormalige Gouverneur der russich-amerikanischen Colonien, $\mathrm{S}^{\mathrm{r}}$ Excellenz Herr Contre-Admiral $\boldsymbol{F}$. W $\boldsymbol{V}_{\text {rangel, }}$ dieser jedem Verehrer der Wissenschaften wohlbekannte Mann, aus Californien mitgebracht und dem Museum der Akademie überlassen hatte. Schliesslich muss ich noch der Güte des Herrn Johannes Oberts, eines jungen hoffnungsvollen Entomologen, erwähnen, mir einige rom Dr. Blaschke gesammelten Käfer aus Sitkha und Californien verabfolgt zu haben.

Da aber Her. Ferdinand Sallberg, Doctor der Medicin und ein würdiger Sohn des berühmten Entomologen, Herrn Professor Sahlbergs, im Jahre 1839 den gegenwärtigen Gouverneur der Besitzungen der russisch-amerikanischen Compagnie, Flott-Capitain ersten Ranges Etholén, dem ich schon längst eine in der Umgegend vou Jakutsk von ihm selbst gemachte Sammlung Käfer verdanke, auf seiner Reise nach Brasilien, Chili und Sitliha begleitete und im Verlaufe dieses Winters nach der Heimath zurückzuerwarten war, entschloss ich mich, meiner vor etwa einem Jahre schon begomnenen Arbeit Einhalt zu thun, und dieses um so lieber, weil Doctor Sahlberg sich ein ganzes Jahr auf Sitkha aufgehalten hatte und deshalb von einem so thätigen und wissenschaft- 
lich gebildeten Sammler recht vieles zu erwarten war. Allein kürzlich wurde von dem Reisegefährten des Herrn Sahlbergs, dem Herrn Wosnesensky, an die Akademie der Wissenschaften eine beträchtliche Sendung von dem letzgenannten auf Sitkha und in Californien gesammelten Insecten heimgeschickt, und die Akademie ertheilte Herrn Ménétriés den Auftrag darüber einen Catalog mit Diagnosen der neuen Arten auszufertigen.-Durch die nehmliche Gelegenheit bekam $\mathrm{S}^{\mathbf{e}}$ Excellenz Herr Fischer von Waldheim eine in denselben Gegenden von einem Zöglinge des Ackerbau-Institutes zu Moskwa, Tschernikh, gemachte KäferSammlung, die mir zur Benutzung für mein Werk gefalligst mitgetheilt worden ist. Um nun nicht meine auf die viele schon fertig liegende vollständige Art-Beschreibungen und die Auseinandersetzung der neuen Gattungen angewandte grosse Mühe, durch den Jald erscheinenden Catalog des Herru Ménétriés zum grossen Theile vereitelt zu sehen, finde ich mich jetzt genöthigt einen Prodromus der von mir begonnenen Käfer-Fauna dem entomologischen Publicum zu überliefern, hauptsächlich in der Absicht um die Priorität der Benennungen der neuen Arten für mich gesichert zu haben.

Durch diese Umstände gezwungen den Druck gegenwärtiger Abhandlung zu beschleunigen , erachte ich es am besten, die im Käfer-Cataloge des 
Herrn Grafen Dejean $\left({ }^{*}\right)$ beobachtete systematische Anordnung zu befolgen, obgleich ich das natürliche System für die Aufstellung in der eigentlichen Fauna zu benutzen gesonnen war, wobei ich auch die Absicht hatte, zugleich eine Darstellung der geognostischen und klimatischen Verhältnisse der gedachten Länder, nebst einigen Bemerkungen über die geographische Verbreitung der daselbst vorkommenden Insecten-Gattungen, als Einleitung zu den Werke, vorangehen zu lassen. Ich beschränke mich also nur auf die blossen Diagnosen der mir bisher bekannten Species und sollte es mir auch nicht später gelingen, meinen aufgefasten Plan durclizusetzen, so wird es mir doch immer zur Genugthuung gereichen, würdigeren Naturforschern den Weg zu eineın vollständigeren Werke über die entomologischen Produkte der mehr erwähnten Länder gebahnt zu haben.

Es enthält also dieser Prodromus Diagnosen von 300 Arten, wovon 148 bisher entweder ganz unbekannt oder in keinem entomologischen Werke beschrieben gewesen sind. Die Species, die ich nicht zur Untersuchung habe bekommeri können, und deren Diagnosen aus verschiedenen Schriften und Abhandlungen abgeschrieben oder auch von mir, ohne Ansicht der

(*) Cataloguc des Coléoptères de la collection de M. le Comte Dejean; troisième édition. Paris, 1837. in- $8^{\circ}$. 
Käfer, ergänzt worden sind, habe ich durch ein * ausgezeichnet. Von denen im Dejean'schen Cataloge angeführten Arten blieben mir doch, ungeachtet alles Strebens sie zur Beschreibung zu exhalten, folgende vierzehn ganz unbekannt, nehmlich aus Californien: Dromius Californicus Dej., Mrcrosaurus confusus $D e j$., Notoxus angustatus Eschsch., Necrophorus lateralis und auripilosus Eschsch., Scotera gibbosa Eschssch., Lixus CaliFornicus Eschsch., Aupuicyrta dentipes Eschsch., Hippodamia 7-lunata Eschesch., Hyperaspis euadrioculata Eschsch., und Rhanis angusticollis Eschsch,, aus Sitkha: Aecialites Debinis Eschsch., Agyrtes latus Eschsch., und Plectrura spinicauda Eschsch., alle diese Arten von Eschscholtz dem Grafen Dejean mitgetheilt, daher es zu vermuthen ist, dass sie in seiner gewesenen eigenen Sammlung sich befinden müssten.

Wiburg den $28^{\text {sten }}$ Februar 1843.

\section{PENTAMERA.}

\section{CARABICI.}

*1. Omus Cabtronnicus Eschscholtz: Niger, nitidus, thorace subcylindrico ruguloso, basi apiceque truncato, elytris connatis ovatis, supra sparsim punctato-rugosis, margine inflexo lavi, linea longitudinali rufo brumnea notato. Longit. $7 \frac{1}{2} \mathrm{lin}$. 


\section{3}

Eschscholtz, Zool. Atlas. I. p. 4. Tab. IV. fig. 1.- Laporte, Annal. Soc. entom. de France I. p. 386. 1., Reiche, ibid. VII. p. 301. 3. pl. 10, fig. 3.-Lacodairc, Révision de la famille des Cicindélides, p. 16.

Habitat in California; ad Cabo de los Reyes mense Novembris sub lapide specimen unicum a D. Eschscholtz captum. Sar thaneive San.

*2. Amblycuema Prcolominir Dupont: Atra, nitida, capite levigato, thorace subquadrato, levigato subcanaliculato, elytris obsolete punctulatis, lineis tribus elevatis, interstitiis punctis profunde impressis

Longit. $13 \frac{1}{2}$ lin. Latit. 4 lin.

Reiche, Annal. Soc. entom. de France. VIIl. p. 560. pl. 19. fig. $1-6$.

Ilabitat in California ad Sinum $\mathrm{S}^{\mathrm{t}}$. Francisco. D. Picolomini.

3. Galerita Caltrornica: Nigro-fusca, testaceo pubescens, capitis puncto, antemnis, thorace angustiore oblongo-cordato pedibusque rufoferrugineis, elytris oblongo-quadratis obscure cyanescentibus, leviter striatis.

Longit. $6 \frac{1}{2}$ lin. Latit. $2 \frac{1}{3}$ lin.

Habitat in California D. Tschernikh.

Gal. Jano Fabr. (cyanipennis Dej. Sp.) minor et in thorace et elytris multo angustior.

4. Cymindrs viridis Eschscholtz: Glabra, viridicyanea, thorace cordato, elytris tenue striato- 
punctatis, punctisque duobus impressis, tibiis tarsisque nigris, antennis basi rufis.

Dejean, Species général des coléoptères V. p. 325. 40. Catalogue des coléoptères $3^{\text {me }}$ édit. p. 9 .

IIabitat in California D. Eschscholtz.

5. Dromius nigrinus Eschscholtz; Obscure nigroæneus, thorace postice angustato, subcordato, elytris obsoletissime striatis, disco impunctatis, antennis pedibusque piceis.

Longit. $1 \frac{1}{2}$ lin. Latit. $2 / 3$ lin.

Dejean Cat. $3^{\text {me }}$ édit. p. 11.

Habitat in California D. Eschscholtz. Mus. Univ. Imp. Mosqu.

Colore D. truncatello Auct. similis, sed thorace elytrisque multo longior.

*6. Lebia cyanipennis Eschscholtz: Nigra, elytris cyaneis.

Longit. 21/2 lin. Latit. 11/4 lin.

Dejean, Spec. gén. V. p. 385, 54. Cat. $3^{\text {mt }}$ édit. p. 12.

Habitat in California D. Eschscholtz.

7. Brachinus Tschernikhit : Ferrugineus, thorace breviore subcordato, angulis posticis rectis, elytris oblongo-quadratis, subcostatis cyaneis, pectoris lateribus abdomineque obscuris.

Longit. 4 lin. Latit. 15/6 lin.

Habitat in California D. Tschernikh.

B. conformi Dej. proximus, sed thorace breviore, angulis posticis rectis et elytris longioribus diversus. 
8. Cychrus axgusticollis Eschschollz: Elongatus, niger, thorace angusto, cordato, elytris obscure violaceo-brumeis, utrinque carinatis, dorso obsolete trilineatis, margine obscure viridi-æneo.

Longit. \&, $91 / 2$ lin. Latit. $3^{1 / 4}, 3^{3 / 4}$ lin.

Fischer, Entomogr. de la Russie II. p. 46. 4. 'Tab. 46. fig.

2. 1II. p. 142. 6. - Dejean, Spec. gén. V. p. 526. 10.

Cat. $3^{\text {me }}$ édit. p. 20.

Habitat in insula Unalaschka D. Langsdorff; in insula Sitkha sat frequens $D D$. Eschscholtz, Kuprianoff et Blaschke; in truncis putridis et sub muscis victitat.

9. Cychrus marcinatus Eschscholtz: Niger, thorace cordato, elytris utrinque carinatis, cupreo-æneis, granulato-striatis, margine viridiaureo.

Longit. 6, 6 $6^{1 / 2}$ lin. Latit. $2^{1 / 2}, 2^{3} / 4$ lin.

Fischer, Entomogr. I. p. 79. Tab. 7. fig. 1. III. p. 142. 5.-Eschscholtz Mém. de la Soc. Imp. des natur. de Moscou VI. p. 98. 2.-Germar Coleopt. spec. nov. p. 3. 5.-Dejean, Spec. gén. II. p. 12. 8. Cat. $3^{\text {me }}$ édit. p. 20.

Habitat in insula Unalaschka, vere sub lapidibus et in rupium fissuris, inter muscos singulatim, D. Eschscholtz; in insula Sitkha frequentius, DD. Eschscholtz, Kuprianoff et Blaschke, in truncis putridis et sub muscis degens.

10. Cichrus vextricosus Eschscholtz: Niger, thorace cordato, postice coarctato, elytris ovatis valde convexis, utrinque carinatis, dense striatis, striis profunde punctatis. 
Longit. 8. lin. Latit. $31 / 2$ lin.

Dejean, Spec. gén. V. p. 527. 11. Cat. $3^{\text {me }}$ édit. p. 20.Eschscholtz Zool. Atlas. V. p. 21. 1. Tab. XXV. fig. 1. IIabitat in California ad St. Franzisco. DD. Eschscholtz et Blaschke.

11. Carabus Chamissonis Eschscholtz: Ovatus, niger, thorace quadrato, postice truncato, elytris subreticulatis, elevato-striatis, striis interruptis, punctisque obsoletis oblongis elevatis triplici serie.

Longit. $6^{3} / 4,7^{1 / 4}$ lin. Latit. $2^{3 / 4}, 3$ lin.

Fischer, Entomogr. I. p. 88. 12. Tab. 7 fig. 12. III. p. 220. 87. - Eschscholtz, Mém. de la Soc. Imp. des natur. de Moscon VI. p. 100. 4.-Dejean, Spec. gén. II. p. 161. 102. Cat. $3^{\text {me }}$ édit. p. 24 .

C. Bracuyderus Wiedemann ín Germar Mag. IV. p. 110, 5 . IIabitat in insula Unalaschka frequens vere sub lapidibus ad littora lacuum. D. Eschscholtz.

12. Carabus baccivonus Eschscholtz: Oblongoovatus, niger, thorace angustato, quadrato, subrugoso, elytris crenato-striatis, interstitiis sæpe interruptis, punctisque impressis triplici serie.

$V a r . b$. elytris brumneo-testaceis. Ledalüs Var. c. punctis excavatis in elytrorum basi deficientibus et seriebus versus apicem sæpe punctis vagis intermixtis.

Longit. $8^{1 / 2}, 9^{1 / 2}$ lin. Latit. $3^{1 / 2}, 3^{3} / 4$ lin. 
Fischer, Entomogr. I. p. 8\%. 11. Tab. 7. fig. 11. III. p. 221. 89.-Eschscholtz, Mém. de la Soc. Imp. des natur. de Moscou VI. p. 99. 3.-Dejean, Spec. gén. II. p. $16 \%$ 10\%. Cat. $3^{\text {me }}$ édit. p. 24.

C. Senratus Wiedemann in Germar, Mag. IV. p. 109. 4.

Habitat in insula Unalaschka frequens per totam æstatem; rapina deficiente flores Prnule cunetfolie Ledeb. depascens, et autumno baccis Emperr nigri vitam sustentans. D. Eschscholtz.

*13. Calosona cancellatum Eschscholtz: thorace brevissimo, scabro, æneo, angulis posticis productis, elytris viridi-aneis, obsolete striatis, rugis Jrevissimis transversis irregularibus , punctisque oblongis elevatis triplici serie, tibiis intermediis rectis.

Longit. 9. lin.

Eschscholtz, Zool. Atl. V.p. 20̄. 4.

Inabitat in California ad $\mathrm{S}^{\mathrm{t}}$. Franzisco. D. Eschscholtz.

14. Leistus ferruginosus $\left(^{*}\right)$ : Rufo ferrugineus, thorace cordato, angulis anticis prominulis, elytris dorso profunde striatis, striis tenui-

(*) Nachdem Germar in seiner vortreflichen Zeitschrift für die Entomologie $2^{\text {tr }}$ B. P. 442. erwiesen hat, dass der Name ferrugineus dem Lcistus spinilabris Fabr. zukommen müsste, habe ich es als unumgänglich erachtet die Benennung der hier fraglichen Art zu ändern.-Was Germar zugleich in Betreff des jezigen Harpalus ferrugineus sagt, muss ich bestreiten, denn ich habe selbst einige Stücke dieses ächten Harpalus im südichen Schweden gefangen. 
ter punctatis, tertia foveolis quatuor impressis.

Longit. $3^{1 / 2}, 3^{3 / 4}$ lin. Latit. $1^{1 / 2}, 1^{2 / 3}$ lin.

Leistus ferrugineus Eschscholt, Dejean. Spec.' gén. V. p.

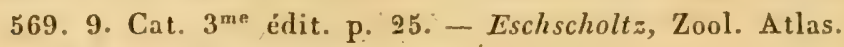
V. p. 24. 9. Tab. XXV. fig. 4.

Ifabitat in insula Sitkha in truncis putridis DD. Eschscholtz, Kuprianoff et Blaschke.

15. Nebria metallica Eschscholtz: Nigra, elytris cupreo-violaceis, striato-punctatis, interstitiis alternatim interruptis.

Var. b. elytris aureo-micantibus.

Longit. $5 \frac{1}{2}, 6$ lin. Latit. $2 \frac{1}{4}, 21 / 2$ lin.

Fischer, Entomogr. I. p. 71. 1. Tab. 6. fig. 1. III. p. 245. 3.-Eschscholtz, Mém. de la Soc. Imp. des natur. de Moscou VI. p. 100. 5.-Dejean, Spec. gén. II. p. 229. 7. Cat. $3^{\text {mic }}$ édit. p. 25.

Ilabitant in insula Unalaschka, vere frequentissima, turbatim sub lapidibus in rupium vicinitate. D. Eschscholtz; in insula Sitkha ad rivulos e montibus defluentes. $D D$. Eschscholtz, Kuprianoff et Blaschke.

*16. Nebria Gebleri Eschscholtz: Nigra, elytris cupreo-violaceis, striatis, striis obsolete punchatis, interstitiis tertio et septimo sub-interruptis.

Longit. $4^{3} / x, 6$. lin. Latit. $13 / 4,2$. lin.

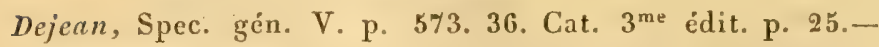
Eschscholtz, Zool. Atlas. V. p. 23. 5. T'ab. XXV. fig. 3. IIabitat in insula Sitkha $x$ state sub lapidibus ad rivulas nivosas in montium vicinịtate rarius $D$. Eschscholtz. 
17. Nebrta cregarta Eschscholtz: Elongata, nigra, elytris nigro-aneis, striatis, striis simplicibus, tertia quadripunctata, antennis pedi'susque piceis.

Var. b. antennis pedibusque totis cum elytrorum margine inflexo rufo-piceis.

Longit. 5 lin. Latit. 2 lin.

Fischer, Entomogr. I. p. 72. 2. Tab. 6. fig. 2 III. p. 250. 10.-Eschscholtz, Mém. de la Soc. des natur. de Moscou VI. p. 101. 6.-Dejean, Spec. gén. II. p. 232. 10. Cat.

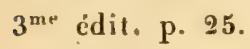

Ilabitat in insula Unalaschka, vere sub lapidibus ad lit tora maris frequentissima. D. Eschscholtz.

18. Nebria Mannerhemin Eschscholtz: Nigra, elytris oblongo-ovatis, striatis, striis obsolete punctatis, punctisque quinque vel sex impressis, antennis pedibusque piceis.

Longit. $4 \frac{1}{2}, 5$ lin. Latit. $1 \frac{3}{4}, 2$ lin.

Fischer, Eintomogr. III, p. 253. 14. Tal. 14. fig. 5.-Dejean, Spec. gén. V. p. 575. 38. Cat. $3^{\text {me }}$ édit. p. 25.Eschscholtz, Zool. Atlas V. p. 23.6.

Habitat in insula Sithka ad rivulas D. Eschscholtz.

19. Nebria Sanlbergil Eschschollz: Nigra, elytris oblongis, striato punctatis, stria tertia punctis quinque impressis, antennis tarsisque rufopiceis.

Var. b. Supra violaceo-micans.

Longit. $3^{5} / 4,4^{1 / 2}$ lin. Latit. $1^{1 / 2}, 1^{3 / 4}$ lin. $N^{\circ}$ II. 1843. 
Fischer, Entomogr. III. p. 254. 16. Tab. 14. fig. 2. - Dejean, Spec. gén. V. p. 576, 39. Cat. $3^{\text {me }}$ édit. p. 25. Eschscholtz, Zool. Altas; V. p. 23. \%.

IIabitat in insula Sitkha cum præcedente D. Eschscholtz; a DD. Kuprianoff et Blaschke, etiam missa, quamvis nullum precedentis speciei individuum communicaverunt.

20. Pelophila Eschscholtzi Sturm: Nigra-ænea, thorace antice elytrorum basi latitudine $x-$ quali, elytris evidenter punctato-striatis, limbo viridi-æneo.

Longit. 5 lin. Latit. 2 lin.

Mannerheim in Hummel, Essais entom, III. p. 40. 4. Fischer, Entomogr. III. p. 260.

Nerria Eschischoltzi Sturm Catalog. p. 173.

Habitat in insula Unalaschka sub lapidibus. D. Eschscholtz.

21. Elaphrus californicus: Supra obscure æeneus, punctatissimus, thorace capitis fere latitudine, sub foveolato, elytris costis subelevatis interruptis, maculisque obscuris ocellatis impressis quadruplici serie; subtus viridi-æneus, femorum basi tibiisque ferrugineis.

Longit. $31 / 4$ lin. Latit. $11 / 2$ lin.

llabitat in California D. Tschernikh

E. Americano Dej. paullo major, capite thoraceque multo latioribus, hoc obsolete tantum foveolato diversus.

*22. Notiophilus syluaticus Eschscholt: Elongatus, supra cupreo-æneus, fronte quinque sulcata, elytris vitta dorsali flava, striisque sex 
dorsalibus remotis, prima dorsali apice fere recta, foveolaque antica impressa.

Longit. $23 / 4$ lin.

Eschscholtz, Zool. Atlas. V. p. 24. 10. Tab. XXV. fig. 5.

N. Bigutratus Var. Dejean, Cat. $3^{\text {me }}$ édit. p. $2 \%$.

Uabitat in insula Sitkha sylvis D. Eschscholtz.

*23. Notiophilus semiopacus Eschischoltz: Supra æneus, fronte multistriata, thorace subquadrato, elytris, sutura, vittaque dorsali tenuiter striata, bifoveolata et apice flavescenti-opacis.

Longit. $2^{5} / 4$ lin.

Eschscholtz, Zool. Atlas. V. p. 25. Tab. XXV. fig. 6.

Habitat in California ad S'. Franzisco. D. Eschscholtz.

24. Metrius contractus Eschscholtz: Niger, thorace quadrato, lateribus late reflexo-marginatis, elytris comnatis, ovatis, convexis, obsolete striato-punctalis, pedibus nigro-piceis.

Longit. $5 \frac{1}{2}$ lin. Latit. $2^{1 / 2} \mathrm{lin}$.

Eschscholtz, Zool. Atlas. I. p. 8. Tab. 1. fig. 4. - Dejean,

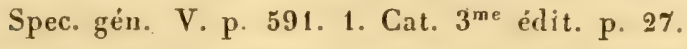

Ilabitat in California, sub lapidibus et truncis putridis arborum D. Eschscholtz.

*25. Loricera semipunctata Eschschollz: Nigropicea, thorace basi parum angustato; angulis basalibus productis, elytrorum striis tenuiter punctulatis, disco tri-foveolato.

Longit. $3^{3} / 4$ lin. Latit. $71 / 2$ lin. 
Esclescholtz, Zool. Atlas. V. p. 25. 12.

Habitat in California ad $\mathrm{S}^{\mathrm{t}}$. Franzisco. D. Eschscholtz.

26. Loricera decempunctata Eschscholtz: Nigroienea, thorace basi subito angustato, angulis rectis productis, elytris punctato-striatis, foveolis quinque, serie interna tribus, externa duabus.

Longit. $31 / 5$ lin. Latit. $11 / 3$ lin.

Eschscholtz, Zool. Atlas. V. p. 25. 13. Tab. XXV. fig. 7 .

Habitat in insula Sitkha DD. Eschscholtz, Kuprianoff es Blaschle.

*27. Chlenius virimifrons Eschscholtz: Capite violaceo, fronte viridi, occipite profunde pun ctato, thorace violaceo, basi coarctato, dense profunde punctato, elytris nigris, obsolete striatis, antennis pedibusque flavo-ferrugineis.

Longit. 7 lin.

Eschscholtz, Zool. Atlas. V. p. 27. 16.

Habitat in California ad St. Franzisco D. Eschscholtz.

"28. Chlennus variabilipes Eschscholtz: Niger, pubescens, capite lævi, thorace subviolaceo, punctatissimo, basi coarctato, angulis productis, elytris obsolete punctulatis, striis profunde punctatis, antennarum basi pedibusque aut rufo-testaceis, aut nigris.

Longit. $5 \frac{1}{2}$ lin.

Eschscholtz, Zool. Altas. V. p. 2\% 1\%.

Habitat in California ad S'. Franzisco D. Eschscholt: 
-29. Culanies uarpalinus Eschescholtz: Capite thoraceque nigro-æneis, nitidis, subviridibus, thorace brevi antice rotundato, medio punctis singulis majoribus impressis, basi in fossis et angulis rudepunctato, elytris nigro-subviridibus opacis, subtilissime striatis.

Longit. $5^{1 / 2}$ lin. Eschscholtz, Zool. Altas. V: p. 2\% 18.

Habitat in California ad S'. Franzisco. D. Eschscholtzo

30. Chlenrus vicinus Dejean: Nigro-piceus, supra pubescens, capite sublævi thoraceque quadrato punctatissimo, viridi-æneis, elytris obscurioribus, striato-punctatis, interstitiis subtilissime granulatis, antennarum basi pedibusque testaceis.

Longit. $4^{3} / 4$ lin. Latit. 2 lin.

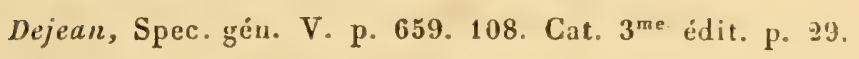

C. purescens Harris.

Habitat in Califormia D. Tschernikl.

$\mathrm{Ab}$ individuis Americanis differt elytrorum interstitiis multo subtilius granulatis; ceterum in omni puncto illis simillimus, quare ut species diversa ægre dijudicandus.

31. Badister ferrugineus Eschscholtz: Capite scu-. telloque nigris, thorace capite latiore rufopiceo, ferrugineo-marginato, elytris fuscis cyaneo-micantibus, margine omni tenue, ano, antennarum basi pedibusque ferrugineis.

Longit. $2^{2 / 3}, 3$ lin. Latit. $1,11 / 4$ lin. 


\section{4}

Dejean, Spec. gén. V. p. 690. 6. Cat. $3^{\text {me }}$ édit, p. 31. Eschscholtz, Zool. Atlas. V. p. 28. 20.

Habitat in California ad St. Franzisco. D. Eschscholtz.

32. Patrobus foveocollis Eschischoltz: Apterus, nigro-piceus, thorace subcordato, postice utrinque foveolato, elytris oblongo-ovatis, subconvexis, punctato-striatis , punctisque quatuor impressis, pedibus rufo-piceis.

Longit. $4^{1 / 1}, 4^{3} / 4$ lin. Latit. $1 \frac{1}{2}, 13 / 4$ lin.

Dejean, Spec, gén. III, p. 30. 4. Cat. $3^{\text {me }}$ édit. p. 32.

Peatysara foveocolus, Eschscholtz. Mém. de la Soc. Imp.

des Natur. de Moscou VI. p. 105, 19,-Fischer, Entomogr, II. p. 129. 2. Tab, 19, fig. 5.

Habitat in insula Unalaschka, sub lapidibus frequens D. Eschscholtz.

33. Patrobus fossifrons Éschscholtz: Apterus, nigro-piceus, thorace subtransverso, subcordato, postice utrinque foveolato, elytris oblongo-ovatis, punctato-striatis, punctisque tribus impressis, pedibus piceis.

Longit, 5 lin. Latit, 2 lin.

Dejean, Spec. gén. III. p. 31. 5. Cat. $3^{\text {me }}$ édit. p. 32.

Platysua fossifrons, Eschscholtz Mém. de la Soc. Imp. des

Natur. de Moscou VI. p. 104. 9.-Fischer, Entomogr.

il. p. 128. I. Tab. 19. fig, 4 ,

Ilabitat in insula Unalaschka sub lapidibus et in Kamschatka etiam occurrit, D. Eschscholtz.

31. Patrobus aterrimus Eschschollz: Alatus niger, depressus, thorace subcordato, postice 
transversim impresso, elytris elongatis, subparallelis, striatis, punctisque tribus impressis.

Longit. $4 \frac{1}{2}$ lin. Latit. $12 / 3$ lin.

Dejean, Spec. gén. III. p. 32. 6. Cat. $3^{\text {me }}$ édit; p. 32.

Ilabitat in insula Sitkha DD. Eschscholtz, Kuprianoff et Blaschke.

35. Calathus ingratus Eschscholtz: Apterus nigropiceus, thorace brevi, subquadrato, leviter convexo, margine rufescente, angulis posticis subrotundatis, elytris oblongo-ovatis, parum convexis striatis, punctisque tribus impressis, antennis pedibusque pallide testaceis.

Longit. $31 / 2,4$ lin. Latit. $11 / 2,1 \frac{5}{4}$ lin.

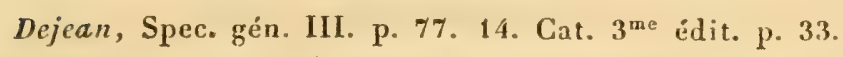

Habitat in insula Unalaschka D. Eschscholt

36. Calathus ruficollis Eschscholtz: Apterus, fusco-piceus, thorace obscure rufo, quadrato depresso, antice subangustato, angulis posticis subrotundatis, elytris ovatis subtiliter striatis, punctisque tribus impressis, antennis pedibusque pallide testaceis.

Longit. $3^{3 / 4}, 4^{1 / 4}$ lin. Latit. $12 / 5,2$ lin.

Dejean, Spec. gén. III. p. 78. 15. Cat. $3^{\mathrm{me}}$ édit. p. 33.

Inabitat in California DD. Eschscholtz, Blasclke et Tschernikh.

37. Calathus Behrensir: Aplerus, fusco-piceus, capite thoraceque brumeo-piceis, thorace quadrato subdepresso, antice vix angustato, 
angulis posticis subrotundatis, elytris ovatis opacis, subtiliter striatis, punctisque tribus impressis, antennis pedibusque ferrugineis.

Longit. 4 lin. Latit. 21/4 lin.

Habitat in California ad Ross D. Dr. Fischer.

Procedenti valde similis et affinis, sed brevior, multo, presertim in thorace, latior et colore capitis thoracisque obscurior.

38. Anchomenus ovipennis Eschscholtz: Apterus niger, subdepressus, thorace cordato, marginato, angulis posticis obtusis, elytris ovalibus apice sinuatis, profunde striatis, punctis tribus minutis impressis.

Longit. 6 lin. Latit. $2^{1 / 2}$ lin.

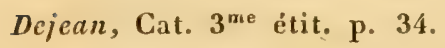

Habitat in California DD. Eschscholtz, Blaschke et Tschernikh; a DD. Fischer de Waldhein et Obert ad describendum benevole communicatus.

39. Anchomenus nugiceps: Apterus, niger subdepressus, capite inæquali rugoso, thorace brevi marginato, posterius angustato, angulis posticis rectis, elytris oblongo-ovalibus, apice sinuatis, leviter striatis, punctis tribus minutis impressis, antennis pedibusque piceis.

Longit. $4 \frac{1}{2}$ lin. Latit: $1 \frac{3}{4}$ lin.

Habitat in California D. Tschernikh.

40. Anchomenus brunneomarginatus: Apterus, niger, depressus, capite lævissimo inter anten- 
nas impresso, thorace longiore, subcordato, lateribus reflexo-narginatis, angulis posticis acutiusculis, elytris oblongo-ovalibus, apice sinuatis, leviter striatis, punctis tribus minutis impressis, antennis, pedibus, thoracis elytrorumque margine rufo-brunneis.

Longit. $4 \frac{1}{2}$ lin. Latit. $13 / 4$ lin.

Habitat in California. D. Tschernikh.

41. Anchomenus californicus Eschscholtz: Alatus, capite, thoraceque obscure viridi-æneis, thorace subquadrato, ely tris nigro-æneis, oblongoovatis, subtilissime striato-punctatis, punctisque quinque impressis, antennis pedibusque piceis.

Longit. $31 / 4,3^{3 / 4}$ lin. Latit. $11 / 4,1 \frac{1}{2}$ lin.

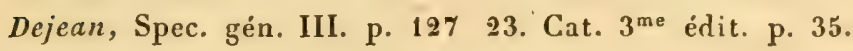
Agonum садifornicum Eschscholtz in litteris. Agonum lepidum Eschscholtz in litteris. Habitat in California DD. Eschscholtz et Tschernikh.

42. Anchomenus ferruginosus Eschscholtz: Alatus, piceus, æneo-micans, thorace oblongo-ovato, elytris, oblongo-ovatis, obsolete striato-punctatis, punctisque quatuor impressis pedibus rufo-pallidis.

Longit. 3 lin. Latit. $1 \frac{1}{4}$ lin. Dejean, Spec. gén. III. p. 128, 24. Cat. $3^{\text {me }}$ édit. p. 35. Agonem ferrugnosum Eschscholtz in litteris. 


\section{8}

43. Anchomenus mollis Eschscholtz: Alatus, ovatus, nigro-piceus, thorace ovato, subquadrato, angulis posticis subrotundatis, ely tris oblongoovatis, subtiliter striatis punctisque duobus impressis, antennis pedibusque rufo-piceis.

$$
\text { Longit. } 4 \frac{1}{2}, 5 \text { lin. Latit. } 1 \frac{1}{4}, 2 \text { lin. }
$$

Dejean, Spec. gén. III. p. 129. 25. Cat. $3^{\text {me }}$ édit. p. 35.

Agonum solle Eschscholtz, Mém. de la Soc. Imp. des Natur. de Moscou VI. p. 102. 7.-Fischer Entomogr. Il.

p. 125. 1. Tab. 19. fig. 2.

Habitat in insula Unalaschka sat frequens sub lapidibus

D. Eschscholtz.

44. Anchomenus sulcatus Eschscholtz: Apterus, ovatus, nigro-piceus, thorace subquadrato, postice utrinque impresso, margine subreflexo, angulis posticis subrotundatis, elytris ovatis, striatis, punctisque quatuor impressis, antennis pedibusque rufo-piceis.

Longit. 4, 5 lin. Latit. $12 / 3,21 / 4$ lin.

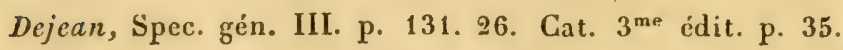

Habitat in California D. Eschscholtz.

45. Anchomenus striatus Eschscholtz: Apterus, ovatus, nigro-piceus, thorace quadrato, angulis posticis subrotundatis, elytris ovatis subtiliter striatis, punctisque quinque impressis, antennis pedibusque rufo-piceis.

Longit. $3^{3} / 4$ lin. Latit. $11 / 2$ lin.

Dejean, Spec. gén. III p. 139. 2\%. Cat. $3^{\text {me }}$ édit. p. 35.

ILabitat in California D. Eschscholts. 
46. Anchomenus maculicorlis Eschscholtz: Apterus, nigro-piceus, ovatus, thorace elytrisque margine late ferrugineo, elytris ovatis, subtiliter striatis, interstitiis obsolete punctulatis, punctisque distinctis sex impressis, antennis pedibusque pallide testaceis.

Longit. $4^{3} / 4$ lin. Latit. 2 lin.

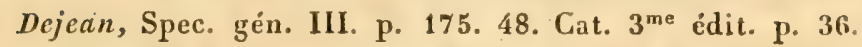

Culenius macubicolus Eschscholtz in litteris.

Habitat in California D. Eschscholtz.

47. Anchomenus brevrcollis Eschscholtz: Apterus, ovalis, niger, thorace breviore, subquadrato, subtransverso, elytris subparallelis, brevioribus, subtiliter striatis, striis obsolete punctatis, punctisque quatuor impressis.

Longit. 4 lin. Latit. 12/3 lin.

Dejean, Spec. gén. III. p. 159. 30. Cat. $3^{\text {me }}$ édit. p. 36 .

Habitat in California D. Eschscholtz.

*48. Anchomenus fossiger Eschscholtz: Niger, thorace oblongo-ovato, elytris elongatis subparallelis, subtiliter striatis, striis obsolete punctatis, punctisque quinque impressis.

Longit. $41 / 4$ lin. Latit. $12 / 3$ lin.

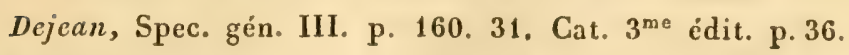

Habitat in California D. Eschscholtz.

49. Pterostichus californicus Eschscholtz: Apterus, niger, thorace subcordato, postice utrin- 
que striato, elytris oblongó-ovatis, subparallelis striatis.

Longit. $5 \frac{1}{2}$ lin. Latit. 2, 21/4 lin.

Feronia (Poecilus) californica Dejean, Spec. gén. III. p. 222. 14. Cat. $3^{\text {me }}$ édit. p. 38 .

Poecilus calfornicus Eschscholtz in litteris.

Bracuystylus californicus Chaudoir, Bullet. de la Soc. Imp. des Nat. de Moscou 1838. p. 17.

Hahitat in California DD. Eschscholtz et Blaschke.

50. Pterostichus vicinus: Apterus, niger, thorace cordato, postice utrinque striato, elytris oblongo-ovatis parallelis, striatis, antennis palpisque brunneo-rufis.

Longit. 5 lin. Latit. $1^{3} / 4$ lin.

Habitat in California DD. Blaschke et Tschernikh.

Præcedenti affinis, sed minor, angustior, thorace antice, latiore, magis cordato, elytrisque thorace angustioribus lateribus omnino parallelis, subtilius striatis diversus.

*51. Pterostichus occidentalis Dejean: Alatus, depressus, supra nigro-cyaneus, thorace lævi, cordato, postice utrinque striato, elytris subparallelis, striatis, punctisque duobus postice impressis, antennarum articulis tribus primis rufis.

Longit. $4 \frac{1}{2}$ lin. Latit. $12 / 3$ lin.

Feronia (Poeclios) occidentalis, Dejean, Spec. gén. III. p. 231. 23. Cat. $3^{\text {me }}$ édit. p. 38.

Poecilus depressus Eschscholtz in litteris.

Habitat in California D. Eschscholtz. 
52. Pterostichus harculaneus: A pterus, niger, thorace anterius parum latiore, depresso, postice utrinque profunde bistriato, elytris ovatis, subparallelis, profunde striatis, humeris denticulatis, apice obtuse rotundatis.

Longit. 8 lin. Latit. 3 lin.

Habitat in insula Sithka; a D. Obert ad describendum benevole communicatus.

Sequenti affinis, sed multo major, magis depressus, thorace longiore utrinque profunde bistriato et aliis notis distinctus.

53. Рterostichus validus Eschscholtz. Apterus, niger, thorace subcordato, postice utrinque striato, elytris oblongo-ovatis, subparallelis , profunde striatis.

Longit. $.5 \frac{2}{3}, 6$ lin. Latit. $2,2 \%$ lin.

Feronia (Platysma) valida, Dejean, Spec. gén. III. p. 325. 116. Cat. $3^{\mathrm{me}}$ édit. p. 40 .

Poecilus validus, Eschscholtz in litteris.

Braciystylus validus, Chaudoir, Bullet. de la Soc. Imp. des nat. de Moscou, 1838. p. 17.

Habitat in insula Sitkha sat frequens; DD. Eschscholtz, Kuprianoff, Blaschke et Tschernikh.

54. Pтenostichus amethystinus Eschscholtz: Apterus, nigro-piceus, nitidus . subdepressus, thorace subquadrato, postice utrinque striato, elytris oblongo-ovatis, subparallelis, profunde striatis, violaceis, antennis pedibusque piceoferrugineis. 
Longit. 5, 61/2 lin. Latit. 12/3, 21/3 lin.

Feronia (Platysma) amethystina, Dejean, Cat. $3^{\text {me }}$ édit.p. 40. Hypierpes ametnystinus Eschschollz, Chaudoir, Bullet. de la Soc. Imp. des nat. de Moscou 1838. p. 13.

Habitat in insula. Sitkha DD. Eschscholtz et Blaschke.

Præcedenti nonnihil affinis, sed magis, præsertim in elytris, depressus, thorace longiore magis cordato et colore diversus.

55. Pterostichus castaneus Eschscholtz: Apterus, nigro-piceus, thorace subquadrato, postice subangustato, utrinque striato, elytris oblongo-ovatis, striatis, antennis, tibiis tarsisque rufo-piceis.

Longit. $4 \frac{1}{2}, 5$ lin. Latit. $1 \frac{3}{4}, 2$ lin.

Feronia (Platysma) castanea Dejean, Spec. gén. III. p. 326.

117. Cat. $3^{\text {me }}$ édit. p. 40 .

IIypheres castaneus Eschscholtz, Chaudoir, Bullet. de la Soc. Imp. des nat. de Moscou, 1838, p. 13.

Habitat in insula Sitkha DD. Eschscholtz et Blaschke.

56. Pterostichus brunneus Eschscholtz: Apterus, , nigro-piceus, thorace longiore, subquadrato , postice subangustato, utrinque striato, elytris oblongo-ovatis, striatis, antennis pedibusque rufo-piceis.

Longit. 41/2 lin. Latit. $12 / 3$ lin.

Fenonia (Platysma) brunnea Dejean, Spec. gén. III. p. 327. 118. Cat. $3^{\mathrm{me}}$ édit. $\mathrm{p} 40$.

Hypherpes brunneus Eschscholtz, Chaudoir, Bullet. de la Soc. Imp. des nat. de Moscou, 1832. p..13. 
Habitat in insula Sitkha DD. Eschscholtz, Kuprianoff et Blaschke, in California haud occurrit, ut allegavit illustris. Comes Dejean.

57. Ptrerosticuus angustus Eschscholtz: Apterus, nigro-piceus thorace elongato, subquadrato, postice sub-angustato, utrinque striato, elytris elongatis, parallelis, striatis, antennis pedibusque rufo-piceis.

Longit. $3^{2 / 3}$ lin. Latit. $11 / 3$ lin.

Feronia (Platysma) angusta Dejean, Spee. gén. III. p. 328. 119. Cat. $3^{\text {me }}$ édit. p. 40.

Anguton angustus Eschscholtz in litteris.

IIabitat in California D. Eschscholtz.

*58. Pterostichus fusco-eneus Eschischoltz: Totus fusco-æneus, capite obscuriore, thorace subtransverso, posterius subito angustato, angulis acutis, utrinque profunde bifoveolato, elytris profunde striatis, interstitio tertio posterius punctis tribus impressis.

Langit, $4^{1 / 2}$ lin. Latit. $1 \frac{1}{2}$ lin.

Omaseus fusco-zeneus Chaudoir, Annales de la Soc. entom. de France, IV. p. 448. 20.

Habitat in insula Sitkha D. Eschscholt .

59. Pterostichus adstrictus Eschscholta: Alatus, niger, thorace subquadrato, postice sub-angustato, utrinque striato, elytris brevioribus, oblongo-ovatis, striatis, striis obsolete punctatis, foveolisque quinque impressis.

Longit. $4^{5} / 4,51 / 4$ lin. Latit. $15 / 4,2$ lin. 
Eschscholtz, Mém. de la Soc. Imp. des nat. de Moscou, VI. p. 103. 8. - Fischer, Entomogr. II. p. 120. 1. Tab. 19. fig. 1 .

Poecilus adstrictus Germar, Col. sp. nov. p. 47. 27.

Feronia (Platysma) adstricta Dejean, Spec. gén. III. p. 319. 110. Gat. $3^{\text {me }}$ édit. p. 40.

Bothropterus adstrictus Chaudoir, Bullet. de la Soc. Imp. des nat. de Moscou, 1838. p. 14.

Habitat in insula Unalaschka sub lapidibus passim D. Eschscholtz; etiam in insula Sitkha DD. Eschscholtz, Kuprianoff et Blaschke.

60. Pterostichus seriepunctatus: Alatus niger, nitidus, thorace subquadrato, postice parum angustato, angulis obtusis, utrinque profunde striato, elytris oblongo-ovatis, subtiliter punctato-striatis, foveolis quinque, omnibus in stria tertia impressis.

Longit. 5 lin. Latit. 2 lin.

Habitat in insula Sitkha D. Blaschke.

Magnitudo et statura præcedentis, sed thorace angustiore, lateribus magis rotundato et fovcolis elytrorum omnibus in stria tertia impressis distinctus.

61. Pterostichus ventricosus Eschscholtz: Apterus, supra æneus, thorace breviore, subquadrato, postice angustato, utrinque striato, elytris ovatis, convexis, striatis, striis subtiliter punctatis, punctisque quatuor impressis, antennis niger, pedibus piceis.

Longit. 41/2, 51/4 lin. Latit. 12/3, 2 lin. 


\section{5}

Poecilus ventricosus Eschscholtz, Mlẻm. de la Soc. Imp. des natur. de Hoscou, VI. p. 106. 11 - Fischer Entomogr. II. p. 132. 1. Tab. 19. fig. 6 .

Feronia (Pratrsma) ventricosa Dejean, Spec. gén. III. p. 329. 120. Cat. $3^{\text {me }}$ édit. p. 40.

Cryobius vevtricosus Chaudoir, Bullet. de la Soc. Imp. des nat. de Moscou, 1838 p. 18.

IIabitat in insula Unalaschka sub lapidibus frequens D. Eschscholtz.

62. Prerostichus pixcuedineus Eschscholtz: Apterus, nigro-aneus, thorace, subcordato, postice utrinque striato, elytris oblongo-ovatis, subconvexis, subtiliter striato-punctatis, punctisque tribus impressis, pedibus rufis.

Longit. 4, 44/2 lin. Latit. 11/2, 12/3 lin.

Poecilus pinguedineus, Eschscholta, Mém. de la Soc. Imp. des nat. de Moscou, VI. 106. 12. - Fischer, Entomogr. II. p. 133. Tab. 19, fig. \%

Feronia (Platrama) pinguedinea Dejean, Spec, gén. 1II. p. 330. 121 . Cat. $3^{\text {mee }}$ édit. p. 40.

Criporus pinguedreus Chaudoir, Bullet. de la Soc. Imp. des nat. de Moscou, 1838, p. 18.

Habitat in insula Unalaschka sub lapidibus passim D. Eschscholt:

13. Pterostichus empretricola Eschscholtz: Apterus, nigro-reneus, thorace cordato, postice utrinque striato, elytris oblongo-ovatis, subtiliter striato punctatis, punctisque quatuor inpressis, antennarum basi pedibusque rufis. Lungit. 31/4, 31/2 lin. Latil. 11/4, 11/3 lin. N. II. 1843 


\section{6}

Feronia (Platysma) empetricola Dejean, Spec. gén. III. p.

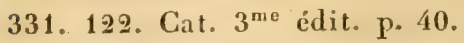

Anchomenus raticollis Sturm, Cat. p. 91.

Gryobrus empetricola Chaudoir, Bullet. de la Soc. Imp. des nat. de Moscou, 1838. p. 18.

Habitat in insula Unalaschka D. Eschscholtz.

64. Pterosticiús riparius Eschscholtz: Apterus, nigro-æneus, thorace breviore cordato, postice utrinque bistriato, elytris oblongo-ovatis subtiliter striato-punctatis, punctisque duobus vel tribus impressis, antennarum basi pedibusque rufis.

Longit. 31/2 lin. Latit. I" lin.

Feronia (Platysma) riparia Dejean, Spec. gén. III. p. 332. 123. Cat. $3^{\text {me }}$ édit. p. 40.

Molops riparius Eschscholtz in littcris.

Habitat in insula Sitkha D. Eschschols.

65. Ptenostichus ater Dejean: Apterus, niger, thorace cordato, postice utrinque bistriato, elytris subparallelis, subtiliter striatis, striis, obsolete punctatis.

Longit. 91/2 lin. Latit. $31 / 2$ lin.

Fero:ia (Pterosticuus) atra Dejean, Spec. gén. III. p. 339. 129. Cat. $3^{\text {me }}$ édit. p. 41 .

Peatysma aterrinum Eschscholtz in litteris.

Habitat in California DD. Eschschollz et IVrangel, specimen. e Museo Acad. Imp. Scient. Petrop. ad examinandum benevole communicavit D. Mínétriés. 


\section{7}

66. Amara litworaiss Eschscholtz: Oblongo-ovata, obscure anea, subliliter punctulata, thorace antice angustato, postice utrinque obsolete biforcolato, foreis punctulatis, elytris striatis, in striis vix punctulatis, antennarum articulis tribus baseos ferrugineis, tibiis tarsisque piceis.

Longit. 3, $3^{1 / 3}$ lin. Latit. $1 \frac{1 / 2}{2} 1^{2 / 3}$ lin.

Zimmermann, in Gistl Faunus I. 1. p. 35. in Silbermann Rexue entom. II. p. 227.

A. plebeja Var. Dejean, Spec. gén. III. p. $46 \%$ \%. Cat. $3^{\text {me }}$ édit. P. 44

A. ronctulata Dejean, Spec. gén. III. p. 472. 14. Cai. $3^{\text {me }}$ édit. p. $44\left({ }^{*}\right)$.

Habitat in insula Sitkha DD. Eschscholtz, Kuprianoff, Blaschlie et Tschernikh.

67. Amara scitcla Zimmermann: Oblongo-ovata, subtiliter punctulata, supra obscure anea, subtus nigro-virescens, thorace anterus angustato, apice emarginato, lateribus non de-

$\left(^{*}\right)$ Dejean hat die A. Littorals Eschsch. mit seinem A. PLEBEsa und punctulata vereinigt, allein nach Zimmermann (Gistl Faunus I. 1. p. 24.) ist diese zulezt genannte eine Varietät der von mitroralis sehr verschiedenen Cela erratica Duftschmid. Die Amaroiden meiner Sammlung wurden alle vor cinigen Jahren von Zimmermann untersucht und benannt, und es befanden sich darunter sowohl die puxctolata aus Kamschatka als dic in Sitkha vorkommende utTorals. 
planatis, hasi utrinclue bifoveolato, foveis punctatis, externa obsoleta, elytris leviter striatis, striis anterius punctulatis, pectore confertim punctato, antennarım articulis tribus baseos ferrugineis, tibiis tarsisque rufo-piceis.

Longit. 31/2 lin. Latit. 11/2 lin.

Zimmermann in Gisll Faunus I. 1. p. 32., in Silbermann Revuc entom. II. p. 223.

Habitat in California D. Eschscholts.

68. Amara insignis Eschscholtz: Ovata, convexa, supra obscure renea, thorace subquadrato, antice subangustato, dorso dievi nitido, postice utrinque bifoveolato, foveis punctatis, elytris, striatis, striis impunctatis, interstitiis subtilissime punctulatis, antennis tibiis tarsisque rufis.

Var. b. supra obscure cyanca.

Longit. $41 / 4,4^{3} / 4$ lin. Latit. 2, 21/3 lin.

Dejean, Spec. gén. V. p. 796. 71. Cat. $3^{\text {me }}$ idit. p. 45. -

Zimmermann in Gistl Faunus I. 1. p. 37. in Silbermann Revue entom, 11. p. 228.

Habitat in California DD. Eschscholtz et Elaschke.

69. Amara remotv stritata Eschscholtz: Subovala, supra obscure anea, thorace brevi, antice subangustato, basi utrinque biforeolato, foveis punctatis, elytris levissime striatis, striis remotis, obsolete punctatis, interstitiis subtilissime punctulatis, pectore distincte punctato, antennis, tibiis tarsisque rufo-piceis. 
Var. b. elytrorum margine inflexo, thorace subtus, pectore abdominis apice, antemnis pedibusque pallide ferrugineis.

Longit. $3^{1 / 3}, 3^{5} / 4$ lin. Latit. 11/2, $1^{3 / 4}$ lin.

Dejean, Spec. gén. III. p. 473. 15. Cat. $3^{\text {me }}$ édit. p. 44.

A. вемота Eschscholtz, Sturm, Cat. p. 91.

Celas remota Zimmermann, in Gistl. Faunus 1.. t. p. 27. in Sillermann Revue entom. II. p. 216.

Habitat in insula Unalaschka D. Eschscholtz; Var. b. c Kamschatka a.D. Dr. Kyber allata.

70. Amara calfonvica Dejean: Oblongo-ovata, supra obscure anca, subtus nigro-virescens, thorace antice subangustato, postice utrinque obsolete hiforcolato, foveola externa oblique evidentiore, elytris leviter striatis, striis impunctatis, sterno pectorali maris foveola oblonga impresso, antennis, tibiis tarsisque rufoferrugineis.

Longit. 4, 41/3 lin. Latit. 13/4, 15/6 lin.

Dejean, Spec. gén. III. p. 474. 16. Cat. $3^{\text {me }}$ édit. p. 44.

A. mapunctata Eschscholtz in litteris.

Cemia catifornica Zimmermann in Gistl Faunus I. 1. p. 25. in Sillermann Revue entom. II. p. 215.

Habitat in Galifornia DD. Eschscholtz et Blaschke.

71. Amara aurata Eschscholtz: Oblongo-ovata, supra anea nitida, subtus nigro-virescens, thorace antice subangustato, postice utrinque bifoveolato, foveis profunde punctulatis, ely- 
tris leviter striatis, striis impunctatis, sterno pectorali integro, antennis ano, pedibustue rufo-ferrugineis.

Longit. 21/2, 3 lin. Latit. 11/4, 11/3 lin.

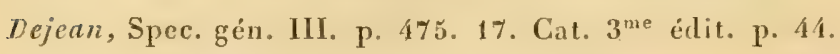

Cema aurata Zimmermann in Gistl Faunus I. 1. p. 28. in Silbermann, Revue entom. P. 218.

Habitat in California DD. Eschscholts, Blaschke et T'scher$n i h h$.

72. Amara meranogastrica Eschscholtz: Oblonga, nigro-picea, supra subnitida, subtus opaca, thorace lateribus rotundatis, postice angustato, utrinque Jistriato, antice posticeque punclato, elytris oblongis profunde punctato-striatis, antennis pedibusque rufis.

$$
\text { Longit. } 4 i_{2}, 5 \mathrm{lin} \text {. Latit. } 13 / 4,2 \mathrm{lin} \text {. }
$$

Dejean, פpec. gén. III. p. 519. 59. Cat. $3^{\text {mo }}$ édit. p. 45.

Leirus mezanogastricus Zimmermann in Gistl Faunus I. 1. p. 33. in Silbermann Revue entom. 11. p. 229.

Habitat in insula Unalaschka D. Eschscholtz.

73. Eripus levissmus Eschscholtz: Niger, nitidus, capite profinde bicanaliculato, mandibulis elongritis porrectis, thorace elongato, posterius angustato, latcribus et postice marginato, clytris ovalibus, obsolelissime striatis, antennis, palpis pedibusque piceis.

$V a r$. b. rufo-picea, pedibus dilutioribus.

Iongit. $4 \frac{1}{3}, 41 / 2$ lin. Latit. $1 \frac{1}{3}, 1 \frac{1}{2}$ lin. 


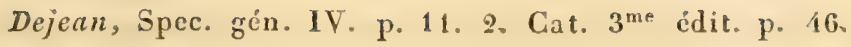
Stomis Levissimus Eschscholtz in litteris.

Habitat in "California rarius D. Eschscholtz.

*74. Anisodactruus californices Eschscholtz. Niger, thorace subquadrato, punctulato, postice angustato, utrinque olosolete foveolato, elytris striatis, interstitio tertio puncto impresso, antennarum articulo primo subtus testaceo.

Longit. 5 lin. Latit. 2 lin.

Dejean, Spec. gén. IV. p. 148. 12. Cat. $3^{\text {me }}$ édit. p. 49.

Harpalus CÁIIFOrnicus Eschscholtz in litteris.

Habitat in California D. Eschscholtz.

\section{DICHEIRUS Eschscholtz.}

Mentum dente medio brevi obtuso.

Palpi articulo ultimo subcylindrico.

Femora antica incrassata; tibire anticæ spina ipicali duplici, interiore lanceolata incurva, intus excavata, interiore minuta.

Tarsi anteriores maris dilatali, subtus setosi, articulo primo triangulari, $2-4$ transversis apice emarginatis.

Caput et thorax profunde punctata, interstitiis elytrorum biseriatim punctatis.

75. Dicheinus Dilatatés Eschscholtz: Oblongo-ovatus, subparallelus, subpubescens nigro-piceus, thorace subquadrato, parcius punctato, postice 
angustato, angulis basalibus obtusis subroinndatis, elytris suiis profunde exaratis impunctatis, antennis pedibusque rufo-piceis.

V́ar. b. capite thoraceque etiam rufescentipiceis.

Longit: $41 / 3,4^{2} / 3$ lin. Latit. $12 / 3,15 / 6$ lin.

Harpalus (Ophonus) dilatatus Dejean, Spec. gén. IV. p 241. 42. Cat. $3^{\text {me }}$ édit. p. 51.

IIabitat in California DD. Eschscholtz, Blaschke et T'schernikh.

76. Dicheirus brunneus Eschscholtz: Oblongus, subparallelus, sulpubescens, nigro-piceus, thorace subquadrato, basi parum angustato, angulis posticis obtusis, dorso crebre punctato, elytris siriis sat profunde exaratis impunctatis, antennis pedibusque rufis.

Longit. 3, 4 lin. Latit. 1, 11/2 lin.

Harpalus (Ophonus) prunneus Dejeans Spec. gén. IV p. 239

41. Cat. $3^{\text {me }}$ édit. p. 51.

Habitat in California DD. Eschscholtz et Tschernik/h.

Cum descriptione ill. Dejean exacte convenit, tantum paullo major et thoracis angulis posticis hand rectis, sed postius obtusis dicandis; an igitur species eadem?

77. Harpalus somunuentus Eschscholtz: Oblongus, niger, thorace quadrato, postice utrinque foveolato, foveis punctatis, angulis posticis obtusis, elytris striatis, postice subsinuatis, interstitio tertio puncto impresso, antemis pedibusque rufis, geniculis nigricantibus. 


\section{3}

Longit. $3^{1 / 2}, 4$ lin. Latit. $1 \frac{1 / 3}{12 / 3}$ lin.

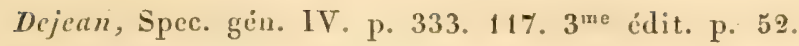

Ilabitat in insula Sitkha DD. Eschscholtz et Blaschke.

78. Harpalus cautus Eschschollz: Oblongo-ovatus, niger, thorace (quadrato, postice utrinque foveolato, angulis posticis subrotundatis, elytris striatis, apice subsinnatis, interstitio terio puncto impresso, antemnarum basi tarsisque rufis.

Longit. $4^{1 / 2}$ lin. Latit. $1{ }^{2 / 3}$ lin.

Dejcan, Spec. gén. IV. 36\%, 143. Cat. $3^{\text {me }}$ édit. p. 53.

Ilabitat in Californi: DD. Eschscholtz et Tschernikh.

79. Marpalus Albionicus: Oblongo-ovatus, nigropicens, thorace transverse-cuadrato, basi punctulato, vix foreolato, angulis posticis rotundatis, elytris tenue striatis, apice subsinualis, interstitio tertio puncto impresso, corpore subtus, antemnis pedibusque ferrugineis.

Longit. 4 lin. Latit. $12 / 3$ lin.

Habitat in Californin ad Ross D. Dr. Fischer.

Statura et magnitudo fore precendentis, sed thorax brevior, basi vix fovcolatus, elytra subtilius striata e? color alius.

80. Harpalus Nigrives Eschscholtz: Oblongus, niger, thorace quatrato, postice utrincure foveolato, angulis posticis rectis, elytris striatis, apice oblique subsinuatis, interstitio tertio 
puncto impresso, antennarum arliculo primo subtus rufo-testaceo.

Longit. $2^{1 / 3}$ lin Latit. ${ }^{3 / 4}$ lin.

Dejean, Spec. gén. IV. p. 399. 16\%, Cat. $3^{\text {me }}$ édit. p. 54.

Trecuus nigrinus Eschscholtz in litteris.

Habitat in insula Sitkha D. Eschscholtz.

*81. Stenolophus unicolor Eschscholtz: Oblongus, supra obscure rufus, capile, pectore, abdomineciue nigro-piceis, thorace subovato, posterius subangustato, utrinque subfoveolato, angulis posticis rotundatis, elytris striatis, interstitio tertio puncto impresso, antennarum bari pedibusque pallide testaceis.

Longit. $21 / 2$ lin. Latit. 1 lin.

Dejcan, șpec. gẻn. IV. p. 411, 4. Cat. $3^{\text {me }}$ édit. p 54.

Habitat in California D. Eschscholtz.

82. Bradycelles Nitidus Eschscholtz: Oblongoovatus, rufo-testaceus, thorace subquadrato, postice utringue subfoveolato, foveis obsolete punctatis, angulis basalibus rectis, elytris obsoletissime striato punctatis, striis suturali marginalique lavigatis profunde impressis, thoracis maculis duabus, elytrorumque macula magna oblonga obsoletis nigro-subcyaneis, antennarum basi pedibuscue pallide testaceis.

Longit. $2^{1 / 2}, 3$ lin. Latit. 1, 11/3 lin. 
Acuparpus nitidus Dejean, Spec. gén. IV. p. 47. 30. Cat. $3^{\text {me }}$ élit. P. 55.

Stenolorius nitidus Eschscholtz in litteris.

IJabitat in California DD Eschscholtz et Tschernikh.

*83. Treches chalybers Sturm: Apterus, nigropiceus, subcyano-micans, thorace subquadrato, postice utrinque foreolato, angulis baseos subrectis, elytris ovatis, striis obsoletissime externis obsoletis, punctisque tribus impressis, antennarum basi pedibusque rufis.

Longit. 2 lin. Latit. 1 lin.

Dejean, Spec. gén. V. p. 17. 11. Cat. $3^{\text {me }}$ édit. p. 56.Sturm, Cat. p. 203.

IJabitat in insula Unalaschka frequens.

84. Inamophonus elfegantulus: Obscure virescenti-rneus, griseo-pilosus, thorace angusto, cordato, angulis baseos obtusis, elytris albidis, depressis ovatis, striis antice frofunde punctatis, postice levigatis, hasi et fascia media transversa undulata, brunneo-testaceis, tibis ferrugineis.

Longit. 2 lin. Latit. ${ }^{3 / 4}$ lin.

Habitat in California D. Tschernıkh.

Palporum articulo tertio apicem versus incrassato ab hoc genere nonnihil recedit, sed ceteris notis ab illo vix separandus; etiam geueri Ciarye Laporte quodammodo adsimilis.

*85. Benibidum indistinctua Eschscholtz: Suprat viridi-aneum, thorace subquadrato, postice 


\section{6}

utrinque bistriato, angulis basalibus rectis, elytris oblongis, tenue punclato-striatis, fasciis undatis macularibus tribus apiceque pallide testaceis, obsoletis, punctisque duobus impressis, antennis basi testaceis, pedibus testaceis æneo-micantibus.

Longit. 2 lin. Latit. ${ }^{3 / 4}$ lin.

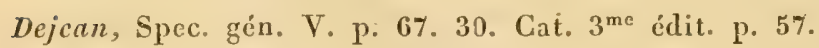

Notapilus indistinctus Eschscholtz in litteris.

Habitat in Calıfornia D. Eschscholtz.

86. Bembidum transversale $=\left({ }^{*}\right)$ Dejean: Capite, thoraceque virili-æneis, thorace subquadrato, postice subangustato, utringue foveolato, angulis baseos rectis, elytris oblongo-ovatis, testaceis, punctato-striatis, fascia lata sinuata viridi-ænea, punctisque duobus impressis, antennarum basi pedibusque testaceis.

Longit. 3 lin. Latit. $11 / 4$ lin.

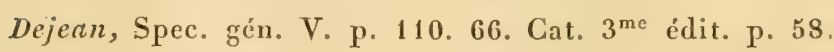

Ilabitat in California D. Tschernikh; specimen ex America boreali occidentali ad ill. Com. Dejean misit D. Leconte.

87. Benbinfum planiusculuat: Nigro-æneum, supra depressum, thorace subquadrato, postice angustato, utringue foveolato, bistriato, margine

(*) Dicse und die folgende vier Arten gehören zu der Abtheilung perypuus. 
viridi-reneo, elytris oblongris deplanalis, profunde striatis, interstitio tertio punctis duobus profunde impressis, antennis pedibusque nigris.

Longit. 2 lin. Latit. $3 / 4$ lin.

IIabitat in insula Sitkha DD. Eschscholts, Kuprianoff et Blaschke. Statura depressa et magnitndo B. Pfeifru Salulberg, sed thorax nonnihil longior, postice magr is angustatus et elytra profundius striata.

85. Bembiniula Kuprianovil . Nigro-aneum, subdepressum, thorace quadrato, utrincue foveolato, bistriato elytris ovatis, subtiliter striatis, interstitio tertio punctis duobus impressis, antennarum basi, tibiis tarsisque rufo-piceis.

Longit. 2\%/ lin. Latit. 1 lin.

Habitat in insula Sitkha D. Kuprianoff.

Procedente majus, latius, thorace breviore, postice vix angustato, elytris brevioribus, subtilius striatis, foveolis in interstitio tertio magis approximatis et colore antennarum pedumque distinctum.

89. Bembidun bimpressum : Nigro-anenm, leviles convexum, thorace subquadrato, postice angustato, utringue foveolato, bistriato, maryine virescente, clytris ovatis brunneo-piceis, leviter striatis, interstitio tertio punctis duobus profunde impresiss, antemis pedibustue piceis.

Longit. 2, 21/2 lin. Latit. 1, 11/3 lin. 
Iabitat in insula Sithka D. Eschscholtz, e museo Univ, Imp. Mosqu. ad describendum benevole communicavit D. Fischer de Waldheim. Proxime pracedenti nonnihil simile, sed paullo majus, convexius, thorace versus basin magis angustato, elytris latioribus magis rotundatis, levius striatis et colore diversum.

90. Bembidum Quadrifoveolatuil : Nigro-reneum, breviusculum, subdepressum, thorace quadrato, utrinque foveolato, bistriato, elytris oblongo-ovatis, subtiliter striatis, interstitio tertio punctis duobus profunde impressis, antennis pedibusque nigris.

\section{Longit. $12 / 3$ lin. Latit. $2 / 3$ lin.}

Habitat in insula Sitkha D. Kuprianoff.

Pracedenti iterum affine, sed longe minus, thorace angustiore, elytrorum foveolis majoribus et colore antennarum pedumque facile distinguendum.

\section{HYDROCANTHARI.}

91. Drtiscus anxius: Oblongo-ovalis, in elytris vix dilatatus, supra nigro-piceus, thoracis limbo omni, elytrorum laterali corporeque pallide flavis, coxarum posticarum laciniis acuminatis aculis.

Longit. 13, 141/2 lin. Latit. $6^{2 / 3} .7$ lin.

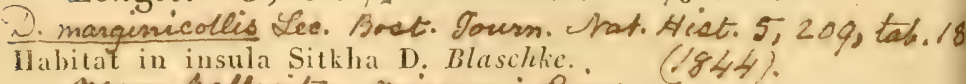

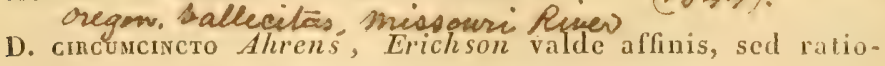
ne longitudinis nomihil angustior, elytris apice pro- 
fundius punctatis et coxarum laciniis minus acutis diversus mihi videtur.

Femina differt a mare tarsis simplicibus, colore minus nitido, thorace magis plano et elytrorum apice fortius punctato

92. Acilius abbraviatus Eschscholl: : Oblongoellipticus, depressus, supra fusco-cinereus, subtus nigro-piceus, undicue dense punctulatus, thoracis limbo vittacue transversa, fronte maculis verticalibus duobus, antenpis, pedibus elytrisque ferrugineis, his creberrime et confertissime nigro-irroratis, fascia transversa postica pallescente ornatis, femoribus posticis ad basin paullulum infuscatis.

Mas: elytris lavibus; Femina: quadrisulcatis, sulcis glabris, prima tantum antice abbreviata.

Longit. 7 lin. Latit. $3^{3} / \mathrm{H}, 4 \mathrm{lin}$.

A. semisulcatus Var. Dejean, Cat. $3^{\text {me }}$ édit. p. 61.-Aubé, Spec. gén. VI. p. 132. 4.

Habitat in insula Sitkha D. Eschscholtz.

A. semsulgato Dej. major, longior et sulcis tribus exterioribus fere ad basin productis species distincta milh videtur, eo magis quod A. Semisulcatus genuinus, quantum mihi innotuit, in insula Sitkha haud fuerit observatus.

93. Colymetes Drvisus Eschscholta: Elongalo ovatus, supra flavicans, subtus niger, thorace in medio maculis duobus rotundatis nigris, elytris crebre nigro-irroratis, prosterno nigro. 
Iar. b. thorace maculis duobus transversis oblongo-quadratis, albdomine toto nigro-piceo, segmentis summo margine vix rufescentibus, pedibus intermediis piceo-maculatis, posticis fere totis nigro-piceis.

Longit. $5^{1 / 3}$ lin. Latit. $2^{2 / 3}$ lin.

Aubé, Spec. gén. VI. p. 248.29.

Rantus Divisus Eschscholtz, Dejean, Cat. $3^{\text {me }}$ édit. p. 62.

Iabitat in insula Sitkha D. Eschscholtz. Var. H. e Museo Univ. Imp̈. Mosqv, ad describendum benevole communicavit D. Fischer de Waldheim.

94. Ilybius euadrinaculatus Aubé: Oblongo-ovatus, minus convexus, posterius rotundatim attenuatus, niger, elytris lineola maculaque minima rotundata rufo-ferrugineis fenestratis.

Var. b. Supra niggro-æeneus.

Longit. 5, $5^{2} / 3$ lin. Latit. $2^{1 / 2}, 2^{3} / 4$ lin.

Aubé, Spec. gén. VI. p. 274. 3.

Colymeetes quadrinotatus Eschscholez, Dejean, Cat. $3^{\text {me }}$ édit. p. 62.

Ulabitat in insula Sitkha D. Eschscholta.

9)5. Agabus tristis Aubé: Elongato-ovalis, sublepressus, vix nitidus, striis anastomozantibus irregulariter strigosus, niger, antennis pedibus marginibusque thoracis et elytrorum rufescentibus.

Longit. $4^{2} / 3$ lin. Latit. 21/4 lin. Aulé, Spec. gén. VI. p. 356. 54. 
Colymbetes picipes Dejean, Cat. $3^{\mathrm{me}}$ édit, p. 62.

Colymbetes angularis Eschschioltz in lilteris.

Ilabitat in insula Unalaschka D. Eschscholtz.

96. Acabus dubius: Oblongo-ovalis, subdepressus, submitidus, striis anastomozantibus irregulariter reticulato-strigosus, brumneo-piceus, antemnis, pedibus, thoracis margine antico et lateribus elytrorumque margine antico et laterali late ferrugineis.

Longit. 41/4 lin. Latil. 21/3 lin.

Habitat in insula Sitkha D. D. ${ }^{\mathrm{r}}$ Siegwald.

Præcedenti proximus, sed multo brevior, posterius magis angustatus, reticulatione minus densa, magis conspicua ut et thoracis elytrorumque margine antico late ferru. gineis precipue diversus.

97. Agabus hypomelas: Ovalis, subconvexus, nitidus, striis anastomozantibus subtilissime reticulato-strigulosus, nigro-aneus, subtus niger, antennis, pedibus, thoracis elytrorumque margine laterali ferrugineis.

Longit. $3^{3} / 4$ lin. Latit. 2 lin.

Habitat in insula Sitkha D. Eschscholtz. Mus. Univ. Imp. Mosqu.

Proxime præcedente multo brevior, posterius haud angustatus, scd elytris versus apicem rotundatis, superficie multo subtilius strigulosa et aliis notis diversus.

98. Hydroponus griseo-striatus De Geer: Elongatoovalis, depressiusculus, vix pubescens, supra testaceo-ferrugincus, subtus niger, ano ferru$N^{\circ}$ II. 1843. 
gineo, capite in vertice el inter oculos nigro, thorace ad latera vix rotundato, angulis basalibus, antice et postice nigro, maculis rotundatis duobus ante basin nigro-notato, elytris lineis septem plus minusve confluentibus, præter suturam angustam, utrinque nigroornatis, lineis sexta et septina abbreviatis et in maculas confluentes interruptis, apice rotundato.

\section{Longit. 2 lin. Latit. 1 lin.}

Aubé, Spec. gén. VI. p. 541. 49.-Dejean, Gat. $3^{\text {me }}$ édit, p. 64.-Stcphens, Illustr. of British Entom. V. p. 391 Manucl of British Colcopt. d. 66. 505.

Hypunnus quadristriatus Eschscholtz, Mém. de la Soc. Imp des natur. de Moscou VI. p. 10\% 13.

Irpirydrus griseostriatús Gyllenhal, Insecta suecica. I. p. 523.7 .

Habitat in aquis insulæe Unalaschkx frequens D. Eschscholtz.

*99. Hydroporus oblongus Dejean: Ovalis, valde elongatus, depressiusculus, punctulatus, subnitidulus, nigro-piceus, capite antice et in vertice vix ferrugineo, thorace ad latera oblique parum rotundato, elytris apice rotundatis, brumneis, vitta transversa ad basin maculaque ad latera ferrugineis, obsolet: ornatis.

Longit. $12 / 3$ lin. Latit. ${ }^{3} / 4$ lin. 
Dejean, Cat. $3^{\text {me }}$ édit. p. 65.-Aubé, Spec. gén. VI. p.. 605. 93.

Hahitat in insula Unalaschka D. Eschscholtz.

100. IIrdroports humeralis Eschscholtz: Oblongo. ovalis, depressus, subtiliter punctulatus, pubescens, supra nigro-brumneis, subtus niger, capite antice et in vertice, thoraceque ad latera fere oblique rotundata obsolete ferrugineis, elytris apice rotundatis, vilta transversa ad basin maculisque tribus irregularibus externis confusis rufo-ferrugineis.

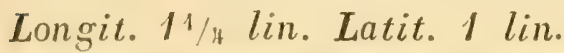

Dejean, Cat. $3^{\text {me }}$ édit. p. 65.-Aubé, Spec. gén. VI. p. 578. 74 .

Habitat in insula Sitkha D. Eschscholtz.

101. Gyrinus picipes Eschscholtz: Ovalis, convexus, coerulescenti-niger, nitidissinus, æueolimbatus, elytris striato-punctatis, striis internis subtilissimis, interstitiis planis lævibus, subtus nigro-æeneus, thoracis et elytrorum margine inflexo nigro-aneo, pedibus ferrugineis, elytris apice truncatis, angulo exteriore rotundato.

Longit. 21/2, 22/3 lin. Latit. 11/4, 11/2 lin.

Dejean, Cat. $3^{\text {me }}$ ćdit. p. 66,-Aubé, Spec. gén. VI. p. 694. $2 \%$.

Habitat in insula Sitkha DD. Eschscholtz, Kuprianoff et Blaschke. 


\section{BR ACHEL Y'TRA.}

102. Iomalota maritima Eschscholtz: Linearis, depressa, nigra, subtiliter griseo-pubescens, antennis brevibus, thorace subquadrato, coleopteris vix angustiore, longitudinaliter impresso, abdomine supra parcius subtiliterque punctato, pedibus fusco-testaceis.

Longit. $1 \frac{1}{3}$ lin. Latit. $1 / 3$ lin.

Habitat in insula Sitkha ad littora maris D. Eschescholtz.

Statura fere Tacuruse, sed tarsi hujus generis.

103. Homalota picipennis: Nigra, tenuiter pubescens, elytris piceo-castaneis, pedibus ferrugineis, thorace subquadrato, vix depresso, basi obsoletius foveolato, abdomine supra anterius parce subtiliterque punctato.

Longit. $11 / 3$ lin. Latit. $1 / 2$ lin.

Hahitat in insula Sitkha DD. Kuprianoff et Blaschke.

H. socranl valde affinis et forsan ejus varietas, sed thorace ànterius minus angustato, basi obsoletius foveolato et antennis brevioribus diversa videtur.

104. Aleochara castaxeipennis Eschscholtz: Nigra, subnitida, parce griseo-pubescens, elytris pedibusque piceo-castaneis, illis thorace longioribus, creberrime punctatis, thorace subrotundato, abdomine sublineari, supra parcius profunde punctato.

Longit $12 / 3$ Iin. Eatit. $2 / 3$ iin. 
IIabitat in insula Sitkha D. Eschschollz.

A. Fomate proxima, thorace anterius haud angustato, elytris longioribus et pubescentia densiore imprimis distincta.

105. Alfochara sulcicollis: Linearis, nigra, subnitida, pilis rigidis parce pubescens, thorace longitudinaliter profunde bisulcato, lateribus sparsim grosse punctato, elytris thoracis longitudine profunde rugoso-punctatis, abdomine parallelo parce punctulato, antemnarum basi pedibusque rufo-piceis.

Longit. $1 \frac{1}{2}$ lin. Latit. $1 / 2$ lin.

Habitat in insula Sitkha D. Eschscholtz; e Mus. Univ. Imp. Mosqu. ad describendum benevole communicatus.

A. mimaculata Gravenhorst minor, angustior, magis linearis, sulcis thoracis profundis et variis notis ab illa separanda.

106. Tachinus Nigricornis: Niger, nitidus, segmentorum abdominis marginibus pedibusque obscure rufo-piceis, thorace lateribus vix rotundato, elytris thorace fere duplo longioribus.

Longit. $2^{1 / 2}$ lin. Latit. 1 lin.

Habitat in insula Sitkha D. Eschscholtz; Mus. Univ. Imp. Mosqu.

T. FLAVIPED Fabr. Erichson nonnihil affinis, sed thoracis latcribus vix rotundatis, elytris multo longioribus, an. tennis brevioribus crassioribus et colore distinctus.

Femina; abdominis segmento supcriorc sexto bifido, la- 
cinia intermedia longiore, latiore, apice acutc acuminata. Mas latet.

*107 Tachinus Frigidus Erichson: Niger, nilidus, antennarum basi, palpis, pedibus thoracisque limbo laterali rufo-ferrugineis, elytris thorace sescui longioribus, piceis, puncto humerali margineque apicali ferrugineis.

Longit. $2 ! / 2$ lin.

Erichson, Gencra et species Staphylinorum p. 256. 21.

IIabitat in insula Unalaschka D. v. Chamisso.

Affinis T, PALLIPEd, RUFIPEDI et FLAVIPEDI operis citati, ab hoc antennis basi rufis, ab illis statura minus convexa et capite thoraceque crebre evidentius punctatis distinguendus.

Mis: abdominis segmento superiore sexto quadridentato, dentibus acutiusculis, intermediis fortiter prominentibus; segmentis inferioribus $\mathbf{3 - 5}$ subimpressis, quinto apice leviter emarginato, margine intra sinum spongioso, sexto bifido, laciniis minus elongatis, compressis modice cur vatis, acuminatis. Femina latet.

108. Tachinus propinques: Nigro-piceus, nitidus, antennarum basi, pedibus, thoracis latioris limbo, elytrisque castaneo-testaceis, his postice infuscatis.

Longit. 2\% lin. Latit. 1 lin.

Ilabital in insula Sitkha DD. Kuprianoff et Blaschke.

T. marginelio Fabr, et Fimetario Grav. affinis, sed utroque major, multo latior et distinctius punctulatus.

Mas: abdominis segmento superiore sexto quadridentato, dentrbus longitudine subrqualibus obtusis, segmento 
inferiore quinto apice medio profunde sinuato, sinu utrinque carinula terminato, sexto profunde inciso, laciniis triaugularibus modice acuminatis.

Femina; abdomine segmento superiore sexto apice quadrifido, laciniis triangularibus, acuminatis.

VAR. b. nigrior, thorace margine laterali et elytris nigris, margine tantum apicali rufescentibus.

109. 'Tacuines elongatus Gyllenhal: Elongatus, nigro-piceus, nilidus, antennis pedibusque rufis, elytris subtiliter substriato-punctatis , margine apicali rufiscente.

Longit. 31/2, 4 lin. Latit. $3 / 1,1$ lin.

Gyllenhal, Insecta suecica II. 251. 1.-Zetterstcdt, Fauna lapponica I. 61. I. Insecta lapponica 56. 1.-Erichson, Genera et Spec. Staphylin p. 265.34.

VAR. b. thorace elytrisque rufo-piceis.

T. FerRugineus Eschscholtz in litteris.

IIabitat in insula Unalaschka DD. Eschscholtz et v. Chamisso; ubi tantum Var. b. lecta.

Mas: abdominis segmento superiore sexto quadridentato, dentibus minutis, intermediis paulo magis prominentibus; segmento inferiore quinto apice late profundeque emargiuato, sexto bifido, laciniis validis, elongatis, compressis, fortius deorsum curvatis, apice acuminatis.

Femina: abdominis segmento superiore sexto apice quadrifido, laciniis subxqualibus, acuminatis.

110. Othies macrocephanus Eschscholtz: Piccus, nitidus, yalpis, antennis, capite thoraceque postice abdominisque segmentorum marginibus rufescentilous, elytrorum dimidia parte 
anteriore cum sutura pedibusque rufo-testaceis, thorace utrinque tripunctato, elytris subtiliter punctulatis, stria suturali impressa.

Longit. 21/2, 31/4 lin. Latit. 1/3, 1/2 lin.

Erichson, Gen. et Spec. Staphylin. p. 279. 5.

Groouypnus macrocepinalus Nordman, Symbolæ ad Monographiam Staphylinorum. p. 124. 26.

Hlahitat in insula Sitkha ad Jittora maris DD. Eschscholtz, Kuprianoff et Blascleke.

Mas: capite thorace fere duplo latiore, quadrato, abdominis ségmento sexto subtus apice emarginato.

Femina : capite thorace vix sesqui latiore, quadrato, abdominis segmento sexto subtus apice rotundato.

\section{Othrus californicus Eschscholtz: Piceus,} nitidus, ore, antemnis thorace basi et apice pedibusque rufescentibus, thoracis seriebus dorsalibus 5-punctatis, elytris subtiliter punctulatis, stria suturali impressa.

Longit. $21 / 3$ lin. Latit. $1 / 2$ lin.

Sauriodes callfornicus? Dejean, Cat. $3^{\text {me }}$ édit p. 72 .

Habitat in California D. Eschscholtz.

O. pilicorsi Payhull, Erichs. similis, sed capite angustiore, thorace elytrisque longioribus ut et punctura thoracis. distinctus.

112. Staphylunus villosus Gravenhorst. Niger, nitidus, thorace antice utrinque cinereo-villoso, angulis posterioribus obtusis, elytris fascia cinerea, subtus pectore abdominisque segmentis quatuor primis cinereo-tomentosis. 
Longit. 7, 9 lin. Latit. 2, 21/2 lin.

Gravenhorst Microphra p. 160. 2. Monog. Micropt. p. 126, 149.-Erichson, Gen. et Spec. Staphylin. p. 319.4.

Creorillus viluosus Nordman Symb. ad Monogr. Staph. p. 21.2.

Eves villosus Dejean, Cat. $3^{\text {me }}$ edit. p. $6 \%$

Habitat in California D, Tschernikh; in America septentrionali, Mexico et Cuba indigenus.

113. Staphylinus eicrictus Eschscholtz: Niger, nitidus, thorace antice vix latiore, ibique utrinque dense cinereo-villoso, angulis posterioribus subrotundatis, elytris fascia angusta cinerea, abdomine subtus segmento secundo tertioque cinereo-tomentosis, pectore nigropubescente.

VAr. b. thorace omnino glabro denudato.

Longit. 7 lin. Latit. 21/3 lin.

Emos viluosus Var. mandibularis Eschsch. Dejean. Cat. $3^{\text {me }}$ édit. p. $6 \%$.

Creopinlus fasciatos? Laporte Etud. entom. p. 111.

Habitat in insula Sitkha DD. Bluschke et Tschernik; Var. b. mihi dedit D. Eschscholtz.

ST. arctico Erichson affinis, sed paullo angustior, thorace antice multo angustiore et antennis crassioribus diversus.

114. Staphylinus tarsalis: Nigro-fuscus, pubescens, capite thoraceque creberrime rugulosopunctatis, carinula longitudinali lævissima notatis, elytris atro-holosericeo-variegatis, ab- 
domine bifariam atro-maculato, tarsis obscure ferrugineis.

Longit. 6 lin. Latit. $1 \frac{1 / 2}{2}$ lin.

Habitat in insula Sitkha D. Tschernikh.

Ad Fam. VIII. Gen. et Spec. Staphyl. cel. Erichsonii adnumerandus.

115. Philonthus californicus Eschscholtz: Niger, nilidus, elytris nigro-æneis, capite subquadrato, thorace lateribus sub-sinuato, utrinque haud impresso, angulis anticis vix deflexis, seriebus dorsalibus quadripunctatis, abdomine subtilius punctulato, cinereo-pubescente.

Longit. 4 lin. Latit. $11 / 2$ lin.

IIabitat in California D. Eschscholtz.

PIr. čNEO, Fabr. magnitudine et statura valde affinis, sed colore, thoracis angulis. anticis vix deflexis, lateribus haud impressís distinctus mihi videtur.

116. Philonthus Siegwaldir. Niger, nitidus, femoribus coxisque anterioribus piceis, thorace seriebus dorsalibus punclis sex profunde impressis approximatis, elytris nigro-æneis, dense et profunde punctatis, capite suborbiculato.

Longit. $21 / 3,3$ lin. Latit. ${ }^{2 / 3}, 3 / 4$ lin.

IIabitat in insula Sitkha DD. Siegwald, Kunprianoff et Blaschke.

Tarsi antice in utrinque sexu simplices. Maris abdomine segmento quinto ventrali apice profunde emarginato, feminæ integro. 
117. Philonthus Albionicus Eschischoltz: Nigger, subnitidus, pedibus cum coxis rufo-piceis, capite ovato, thorace seriebus dorsalibus punctis septem impressis, lateribus parce punctato.

Longit. $31 / 4$ lin. Latit. $3 / 4$ lin.

IIabitat in California D. Eschscholts.

Habitu fere PII, micantis Grav. sed major et variis notis al, illo distinguendus.

118. Quedius plagiatus: Niger, nitidus, thoracis margine antico, segmentorum abdominis marginibus, pedibus elytriscue rulis, his glabris sublevibus, in utroque plaga magna nigrofusca, thoracis seriebus dorsalibus tripunctatis.

Longit. $3^{5} / 4$ lin. Latit. ${ }^{3} /{ }^{4}$ lin.

Habitat in insula Sitkha D. Eschscholtz; Mus. Univ. Imp. Mosqu.

Qv. Levigato Gyllenhal valde affinis, sed thorax angustior, lateribus multo minus rotundato-dilatatis et color alius.

119. Quedius pediculus Eschscholtz: Niger nitidus, thoracis seriebus dorsalibus tripunctatis, elytris biseriatim punctatis, piceo-marginatis, fronte inter oculos quadripunctata.

Longit. 3, $3^{3} / 4$ lin. Latit. $3 / 4,1$ lin.

Erich on, Gen. et Spec. Staphyl, p. 531. 9.

Prilontius pedicuius Nordmun, Symb. ad Mouogr. Staphyl. p. 79.24. 
IIahitat in insula Unalaschka DD. Eschscholtz et v. Chamisso; fieruentissimus in capillis Aleutorum adipe piscario vel phocæo immunde imbutis, unde denominatio.

120 . ()ededus brunntpennis: Nigro-æeneus, subnitidus, elytris parce profunde punctatis, marginibusque segmentorum abdominalium rufobrunneis, thoracis seriebus dorsalibus tripunctatis, fronte inter oculos bipunctata.

Longit. $2^{3 / 4}$ lin. Latit. $2 / 3$ lin.

Ilabitat in insula Sitkha D. Eschscholtz, Mas. Univ. Imp. Mosqu.

1.21. Quedius nolocmus Gravenhorst: Niger, nitidus, antennis pedibusque rufo-piceis, elytris thorace brevioribus, castaneis, scutello punctulato, thoracis seriebus dorsalibus tripunctatis.

Longit. 4, 5 lin. Latit. 1, 1/1/4 lin.

Erichson, Gen. et Spec. Staphyl. p. 535. 18. Käfer der Mark Brand. I. p. 489.8.

Stapirunus mozocinus Gravenhorst, Monogr. Micropt. P. 46. 6:-Gyllenhal Insecta suecica II. p. 302. 20.Sallberg, Insecta fennica I. p. 314. 1\%.-Mannerheim, Précis des Brachelytr. p. 25. 28.-Runde, Brachel. spec, p. 5. 21.

Emus molochnus Boisduval et Lacordaire, faune entom.des envir. de Paris I. p. 37\%. 28.

Panlontules molocunus Nordman, Symb. ad Monogr. Staphyl. p. 76. 6. 


\section{3}

Stapuylues laticolus Gravenhorst Micropt. Brunsv. p. 173. 28. Mon. Micr. p. 47. 8.

Stapir. picipennis Paykull, Fauna suecica III. p. 373. 2.

Stapi. uappunicus Zetterstedt, Insceta lapponica p. 61. 12.

Stapir. Lievcoltis Runde Brachel. spec. P. 6. 21.

Microsaurus molocmunes Dejean, Cat. $3^{\text {me }}$ p. 69.

Habitat in insula Sitkha D. Eschscholtz, Mus. Univ. Inp. Mosqu., in omni Europa et America scptentrionali obvius.

*122. Quedius hyperboreus Erichson: Niger, pedibus anterioribus cum coxis antennisque piceo-testaceis, thoracis sericbus dorsalibus tripunctatis, elytris thorace brevioribus, nigrosubæneis, subtiliter punctulatis.

Longit. 3 lin.

Erichson, Gen, et Spec. Staphyl. p. 547. 35.

Habitat in insula Unalaschka D. Chamisso.

123. Oxytelus fuscipenis : Niger, nitidus, antennarum basi pedibusque testaceis, elytris brunneo-piceis, thorace trisulcato, fronte punctulata, antice impressa lævique.

Longit. $12 / 5$ lin. Latit. $2 / 3$ lin.

Habitat in insula Sitkha DD. Kuprianoff et Blaschke.

O. Luteipensi Erichson (pice Gyllenhal, Mannerh.) proximus, sed minor, fortius punctatus, frontis sulcis deficientibus et colore elytrorum diversus.

Abdominis segmentum inferius sextum maris integram, septimum leviter bisinuatum, lobo intermedio in mucronem parvum producto. Femina latet. 
124. Anthophagus laticollis: Niger, pubescens, crebre subtiliterque punctulatus, fronte lineis duabus obliquis profunde impressis in vertice commexis, thorace brevi lato subquadrato, subdepresso, ad angulos utrinque impresso, medio tenue canaliculato.

Longit. $12 / 3$ lin. Latit. ${ }^{3 / 4}$ lin.

Habitat in insula Sitklia D. Kuprianoff.

A. plagrato Paykull colore subsimilis, sed nigrior, obscurior et thoracis structura ab omnibus congeneribus distinguendus.

125. Arpedium testaceun Eschscholtz: Pallide testaceum, parum nitidum, capite pectoreque nigris, oculis magnis prominulis, capite thoraceque subquadrato crebre subtilissime punctulatis, hoc medio canaliculato, lateribus late explanato, elytris fortius profundiusque punctatis.

VAR. b. abdomine etiam nigro.

Longit. $1 \frac{1}{2}, 2$ lin. Latit. $2 / 3,1$ lin.

Heterops testaceus Eschscholtz in litteris.

IIabitat in insula Sitkha DD. Eschscholtz, Kuprianoff et Blaschke.

126. Arpedium inacultcolde: Nigrum, parum nitidum, antennarum basi, thorace elytris pedibusque pallide flavo-testaceis, thorace subquadrato, lateribus late explanato, dorso medio nigro, elytris fortiter transversim rugosis. 
Longit. $13 / 4$ lin. Latit. $3 / 4$ lin

Ilabitat in insula Sitkha D Tschernikh.

127. Omadem pragratem: Nigrum, nitidulum, antennis pedibusque testaceis, elytris antrorsum? plaga utrinque magna oblicfua rufo-ferruginea, capite thoraceque creberime subtilissime, elytris paulo fortins punctatis.

Longit. $11 / 2$ lin. Latit. $2 / 3$ lin.

Habitat in insula Sitkha D. Eschscholtz. Mus. Univ. Imp. Mosqu.

128. Anthobium fimfarang Eschscholtz: Depressum, rufo-piceum, subnitidum, crebre punctatum, antennarum basi, thoracis elytrorumque limbo pedibusque pallidioribus, capitc nigro, thorace transverso canaliculato, elytris substriatis, ahdomen fere totum obtegentibus, maris truncatis, femine apice interiore sinuato-acuminatis.

Longit. $1 \frac{1}{2}, 2$ lin. Latit. $3 / 4,1 \frac{1}{3}$ lin.

Erichson, Genera et Spec. Staphylin. p. 899; 19

Omaliem rimetarium Munnerh. Précis des Brachel. p. 59.10 Habitat in insula Sitkha D. Eschscholtz.

129. Anthoвiun Pothos Eschscholtz: Rufo-testaceum, capite thoraceque transverso canaliculato subtiliter punctulatis, elytris abdomine parum brevioribus profunde punctatis, apice in utroque sexu truncatis, abdomine maris nigro. 
Longit. 1, 11/3 lin. Latit. $2 / 3,3 / 4$ lin.

Ilabitat in insula Sitkha DD. Eschscholtz, Kupriunoff et Blaschke.

A. ophtialmco Paykull majus, convexius et abillo in multis diversum.

\section{STERNOXI}

*130. Apatura Drummondr Kirby: Supra nigroænea, subtus ænea, nitida; prothorace varie acuducto disco fere concentrice; elytris punctulatis integerrimis, guttis tribus luteis triangulo ordinatis.

VAR. B. Elytris maculis quatuor luteis : 2, 1,1 .

Longit. 5 lin. Latit. $1^{3 / 4}$ lin.

Laporte et Gory Monographic des Buprestides, $\Lambda$ patura p. 3. Tal. 1. fig.3.

Buprestis (Trachypteris) Druamondr Kirby in Richardson Fauna boreali-americana IV. p. 159. 11. Tab. 1I. fig. 2. Var. b.

Buprestis discopunctata? Faldermann, Bullet. de la Soc. Imp. des natur. de Moscou VI. p. 4\%. Tab. 2. fig. 5.

Apatura discopenctata? Laporte et Gory 1. c. p. 3. Tab. 1. fig. 2.

Buprestis guttulata? Gebler, Mém. de la Soc. Imp. des natur. de Moscou VIII. p. 41. 5.

Puenops Gebueri ? Dejean, Cat. $3^{\text {me }}$ édit. p. 89.

Habitat in California et in Rocky Mountains Amer, bor. Kirby; etiam in Mongolia et Sibiria, si Bupr. discopurctata cadem sit species. 
131. Buprestis nusticonul Kirby: Nigro-ænea, nitida, fronte, genis rentrisque lateribus fulvomaculatis, elytris granulatis profunde striatis, apice rotundatis.

Longit. 9 lin. Latit. $31 / 4$ lin.

Laporte et Gory, Monographie des Buprest. Suppl. p. 117. Tab. 20. fig. 115 .

Buprestis (Avoris) rusticorum firby in Richardson Fauna boreali-americana IV. p. 151.1.

VAR. b. segmento ultmo ventrali basi transversim sanguineo, reliquis immaculatis, cxtera ut in a.

IIabitat in Cialifornia. Specimen a D. Amirali trangel, allatum in Mus. Imp. Acad. Scient. Pctropolit. asservatur.

132. Buprestis Langh: Oblonga, depressa, viridiaurea, capite thoracisque disco cocrulescentibus, capite rugoso, thorace profunde punctato, elytris dense costatis, interstitiis rugulosis, apice truncalis, muticis.

Longit. $81 / 2$ lin. Latit. 3 lin.

Habitat in insula Sitkha D. Blaschke.

*133. Anthaxia eneogaster heiche: Supra nigropurpurea, thorace lateribus rotundato, elytris punctis rotundatis impressis, corpore subtus pedibusque nigris, nitidis.

Longit. $21 / 2$ lin. Latit. $11 / 3$ lin.

Laporte et Gory, Monogr. des Buprest. Anthaxia p. 32. Tab. 7. fig. 44.

Habitat in California.

$N^{\circ}$ II. 1843. 


\section{8}

*134. Epiphanis connutus Eschscholtz: Rufo-ferrugineus, capite nigro, antennis articulis quatuor ultimis elongatis $\left({ }^{*}\right)$, fronte cornu compresso, thorace transverso nigro, angulis posticis ferrugineis.

Longit. $21 / 4$ lin.

Eschscholtz, Zoolog. Atlas. I. p. 10. Tab. IV. fig. 6. - In Thon Entomologisches Archiv. II. 1. p. 35.-Laporte in Silbermann, Revue cutom. III. p. $17 \%$.

IIabitat in insula Sitkha rarissime, specimen unicum prope truncum vetustum Pini volitans cepit D. Eschscholtz.

*135. Cardiophonus uatiusculus Eschscholtz: Niger, frontis margine reflexo, thorace lato canaliculato, fusco-tomentoso, pedum geniculis ferrugineis.

Longit. $3^{3} / 4$ lin.

Eschscholtz in Thon, Entomol Archiv, II. 1. p. 34.

Habitat in California D. Eschscholt

136. Cardiophorus califonnicus: Elongatus niger, punctatissimus, tenue pubescens, thorace convexo, subquadrato, elytris dorso depressis, leviter punctato-striatis, sterno profunde punctato, convexo, tarsis articulis omnibus et unguiculis simplicibus.

$\left(^{*}\right)$ Die Beschreibung der Fühlhörner, als zur. Characteristik der Gattung gehörend, wird in der vollständigen Fauna, wo diese Charakteristik besonders auseinander gesclzt wird, aus der Art Diagnose weggelassen werden. 
Longit. $5^{1 / 3}, 4^{3} / 4$ lin. Latit. $12 / 3,13 / 4$ lin.

Habitat in California DD. Blaschke et Tschernikh.

137. Cardiophorus tantillus: Oblongus, niger, fusco-pubescens, profunde punctatus, thorace co.-vexc, lateribus haud dilatato, elytris profunde punctato-striatis, tarsorum articulo quario appendiculato, unguiculi simplicibus

Longit. 2 lin. Lalit. ${ }^{3 / 4}$ lin.

Habitat in California D. Eschscholtz.

138. Cryptohypates nocterivus Eschscholtz: Supra nigro-aneus pilosus, thorace planiusculo, medio dilatato punctulato, elytris simpliciter striatis, in interstitiis punctulatis, antennis nigris, pedibus fuscis, tarsis articulis simplicibus.

Longit. 3, 31/2 lin. Latit. 11/4, 11/2 lin.

Dejean, Cat. $3^{\text {me }}$ édit. p. 105.

Hypontums nocturnus Eschscholta in Thon Entom. Archiv. II. 1. p. 33 .

Habitat in insula Sitkha DD. Eschscholls, Fuprianoff et Blaschlie.

*139. Сияртонypnus musculus Eschscholtz: Brevis, reruginosus, pubescens, thorace lato rotundato, convexo, elytris striatis, interstitiis rugulosis, pedibus flavis, geniculis fuscis, tarsis articulis simplicibus,

Longit. $11 / \mathrm{s}$ lin. 
Hrontrinos mosculus Eschscholtz in Thon Entom. Archiv. II. 1. $\mathrm{p}, 33$.

Erater musculus Eschscholtz, Entomograplien p. 70. 42. Habitat in insula Unalaschka D. Eschscholtz.

140. Cryptonypnes cinereipennis Eschscholtz: Nigro-fuscus, thorace brevi, medio dilatato, convexo, parcius punctulato, sæpe angulis omnibus ferrugineis, elytris striatis brunneo-piceis, tarsis articulis quarto appendiculato.

Longit. $2 \frac{1}{4}, 4 \frac{1}{2}$ lin. Latit. 1, 2 lin.

Monochepidies cinereipennis Eschscholtz in Thon Entom. Archiv. II. 1. p. 32.-Dejean, Cat. $3^{\text {me }}$ édit. p. 98.

Ilabitat in Califarnia DD. Eschscholtz et Sieguvald.

141. Cryptohypeus peberulus: Nigro-fuscens, griseo-pubescens, thorace punctatissimo, lateribus vix dilatato, angulis posticis rufescentibus, elytris punctato-striatis, basi rufescentibus, tarsis articulo quarto appendiculato.

Longit. $1^{3 / 4}$ lin. Latit $3 / 4$ lin.

Habitat in California D. Tschernikh.

Pracedente panllo minor, pubescentia et punctura multo densiore, thoraceque angustiore diversus.

142. Elater carbonicolor Eschscholtz: Ater, angustus, nigro-pubescens, thorace longiore subtiliter punctato, elytris profiunde punctatostriatis, antemnis nigris, pedibus fuscis.

Longit. $3 \frac{1}{2}$ lin. Latit. 1 lin.

Eschscholtz, in Entomol. Archiv. II. 1. p. 33. 
Ampeus carbonicolor Dejean, Gat. $3^{\mathrm{mm}}$ édit. p. 105.

Habitat in insula Sitkha DD, Eschscholtz, Kuprianolf et Blaschke.

*143. Diacantuus resplendens Eschschollz: Cupreo-æneus, nitidissimus, glaber, elytris viridibus, sparsim et subtilissime punctulatis, thorace subquadrato punctatissimo.

Longit. 6 lin.

Ludius resplendens Eschscholt in Thon Entom. Archiv. IJ.

1. p. 34.-Dejean, Cat. $3^{\text {me }}$ édit. p. 107 .

Habitat in insula Sitkha D. Eschscholtz.

144. Diacanthus angusticolirs: Elongatus, nigroaneus, griseo-pilosus, thorace elongato profunde punctato, angulis posticis productis, divaricatis, apice truncatis, elytris punctatostrialis, margine pedumque geniculis rufoferrugineis.

Longit. 6 lin. Latit. $13 / 4$ lin.

Habitat in insula Sitkha D. Blaschke.

145. Diacanthus serricorxis: Elongatus, fusco-testaceus, punctalissimus, griseo-pubescens, thoracis angulis posticis valde productis divaricatis, elytris striatis, antennis serratis, corpore subtus pedibusque piceo-testaceis.

Longit. 4 lin. Lalit. $11 / 2$ lin.

Habitat in California D. Tschernikh.

*146. Diacantuus volitans Eschscholta: Niger, tenue tomentosus, thoracis lateribus, elyiris 
pedibusque rufo-ferrugineis, thorace elongato, densissime punctulato, lateribus explanato.

VAr. b. elytris brunneis.

Longit. $7,8^{1 / 2}$ lin.

Ludies volitans Eschscholtz in Thon Entom. Archiv. II. I p. 34.-Dejean, Cat. $3^{\text {me }}$ édit. p. $10 \%$

Habitat in insula Sitkha D. Eschschalt

*147. Diacantules sagitticollis Eschscholtz: Niger, flavo-pilosus, elyiris pedumque geniculis rufoferrugincis, thorace ciytris angusiore, punctatissimo, angrilis posticis spinis angustis divaricatis.

\section{Longit. 6 lin.}

Ledus EsGitricontis Eschscholt in Thon Entom. Archiv. II. 1. p. 34.-Dejean, Cat. $3^{\text {me }}$ édit. p. $10 \%$

Hábitat iả Sitkha D. Eschsclooltz.

148. Dezcative umbricola Eschscholtz: Elongatus, niger, cinerec-pilosus, thorace elongato, lateribes densichme punctato, in medio sparsim puncinlato, basi angustato, angulis latioribus diraricatis rufis, antennarum articulo tertio elongato.

VAr. b. angulis posticis thoracis fuscis. Longit. 5, 61/2 lin. Latit. 11/2, 12/3 lin.

Ludius umprcola Eschscholtz in Thon Entom. Archiv. II. 1. p. 34.-Dejean, Cat. $3^{\mathrm{m} \theta}$ édit: p. $10 \%$.

Ilabitat in insula Sitkha DD. Eschscholz, Kuprianoff et Blaschie. 
149. Diacanthus lobatus Eschscholtz: Oblongus, niger, subæneus, einereo-pubescens, thorace elongato sublineari, angulis posticis productis divaricatis, apice ipso truncatis, lobo medio marginis postici emarginato, antennarum articulo tertio elongato.

Longit. $4 \frac{1}{2}$ lin. Latit. $1 \frac{1 / 2}{2} 12 / 3$ lin.

Elater lopatus Eschscholls Entomographien p. 69. 41. (ㅇ).

Ludius robatus Eschscholta in Thon Entom. Archiv. II. 1. p. 34 .

Ludius canicinus Eschscholtz, Dejean, Cat. $3^{\text {me }}$ édit: p. 107. ( $\delta)$.

IIabitat in insulis Unalaschka et Sitkha DD. Eschscholtz et Kuprianoff.

*150. Dincantuus diversicolor Eschscholla: Ater, glaber, nitidus, thorace rufo, convexo, vage punctulato, tenue canaliculaio, basi coarctato, angulis posticis acutis nigris, elytris nigroæneis.

Longit. $5 \mathrm{lin}$.

Ludus divensicolor Eschscholtz in Thon Entom. Archiv. II. 1. P. 34 .

IIabitat in California D. Eschscholtz.

151. Dolopius Camifornices Dejean: Elongatus, fuscus, pubescens thoracis marginibus omnibus, elytrorum sutura, antemnis pedibusque fusco-ferrugincis, thorace punctatissimo, elytris striatis, interstituis subtiliter transversim rugulosis. 


\section{4}

Longit. $31 / 2$ lin. Latit. 1 lin.

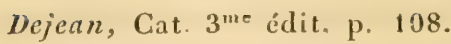

Dolopius hatenalis Eschschaltz in Thon, Entom. Archiv.

II. 1. $\mathrm{p} 34$

Ilabitat in California DD. Eschscholtz et Tschernikh.

152. Athous ferruginosus Eschscholtz: Elongatus, fuscus, elytris, abdomine, antennis pedibusque rufo-ferrugineis, thorace elongato punclatissimo, angulis posticis ferrugineis, maris antrorsum angustato, angulis posticis nonnihil productis, femina lateribus parallelis, an$\mathrm{gulis}$ posticis obtusis magis divaricatis; elytris punctatis leviter punctato-striatis.

Var. b. rufo-ferrugineus, capite nigro-fusco.

Var.c. fusco-piceus, antennis, elytrorum margine, geniculis, tibiis tarsisque ferrugineis.

Longit. 4, 6 lin. Latit. 1, 11/2, lin.

Eschscholtz in Thon Entom. Archiv. II. 1. p. 33.-Dejean, Cat. $3^{\text {nie }}$ édit. p. 102.

Ilabitat in insula Sitklsa DD. Eschscholtz, Kuprianoff et Blaschke.

153. Athous paliddipennis: Elongatus, niger, griseo-pubescens, elytris ferrugineo-testaceis punclatis, obsolete punctato-striatis, thorace oblongo, antrorsum angustato, punctato, obsalete canaliculato.

Longit. 4 lin. Latit. $11 / 3$ lin.

Habitat in insula Sitkha D. Blaschke. 


\section{5}

A. Scrutatom Herlst colore similis sed minor et multo angustior.

*154. Athous nufiventris Eschscholtz: Ater, fionte excavata, thorace punctatissimo, angulis basi planis, elytris castaneis, abdomine pedibusque rufo-ferrugineis.

Longit. $51 / 2$ lin.

Eschscholtz in T'/2on, Entom. Archiv. II. 1. p 33.

Euater rufiventris Eschscholtz, Entomographien p. 71.43. IIabitat in insula Unalaschka D. Eschscholtz.

\section{MALACODERMI.}

155. Dictyopterus inatatus Eschscholtz. Valde elongatus, sanguineo-coccineus, subtiliter pubescens, thorace brevi, obsoletius reticulatocostato, elytris costatis, interstitiis latioribus bifarian reticulatis, antenuis elongatis subserratis abdomineque nigro, coxis posterioribus productis acutis.

Longit. 5, 7 lin. Latit. 12/5, 21/3 lin.

Dejean, Cat. $3^{\text {me }}$ édit. p. 112.

Ilabitat in insula Sitkha DD. Eschscholtz et Blaschke.

156. Dictyopterus simplicipes Eschscholta: Elongatus, sanguineus, thorace longiore, elevatius reticulato-costato, medio infuscato, elytris costatis, interstitiis angustioibus, bifariam reticulatis, antennis brevioribus crassis sub- 
setaceis, corpore subtus coxis hatd productis tarsisque nigris.

Longit. 5 lin. Latit. 2 lin.

Dejean, Cat. $3^{\text {me }}$ édit. p. 112.

Ilabitat in insula Sitkha D. Eschscholtz.

Pracedente multo minor, brevior, thoracis longioris structura et coxis inermibus mox distinguendus.

15\%. Rinagonycita piniphima Eschscholtz: Lineariclongata, nigra, pubescens, mandibulis, palpis, antennarum articulis tribus baseos subtus obscure testaceis, thorace longiore subquadrate, dorso canaliculato, utrinque longitudinaliter subcarinato.

Longit. $21 / x, 31 / 2$ lin. Latit. $2 / 3,1$ lin.

Eschscholtz, Bulletin de la Soc. Imp. de Moscou II. p. 65.

Podatrus pinipmitus Dejean, Cat. $3^{\text {me }}$ édit. p. 118.

IIabitat in insula Sitkha DD. Eschscholtz, Kuprianoff et Blasclke.

158. Cantharis rotata: Nigro-fusca, pubescens, fronte, ore, antcmis, thorace oblongo-quadrato, pedibus abdominisque margine rufoferrugineis, thoracis disco macula parva undulata nigra, elytris rugoso-punctulatis, elevato-lineato.

Longit. 4 lin. Latit. $11 / 1$ lin.

Habitat in California D. Tschernikh.

159. Silis Pallida Eschscholtz: Elongata, nigra, mandibulis geniculisque pallidis, thorace ely- 
triscpue luteo-testaceis, nigro-limbatis, thorace transverso inæcuali postice utrinqu? profunde exciso, angulo basali hamato.

Longit. 2, $2^{3} / 4$ lin. Latit. ${ }^{3} / \mathrm{H}, 11 / 4$ lin.

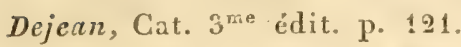

II abitat in insula Sitkha DD. Eschschollz, Kuprianoff et Blasclike.

160. Collops mistrio Eschscholtz: Niger, capite antice antennarumque basi testaceis, thorace rufo, elytris testaceis maculis duabus nigro-virescentibus.

Mas: antennarum articulo primo incrassato, tertio maximo iregulari intus concavo, tarsis anticis 4 -articulatis.

Femina: antennarum articulis 1 et 3 reliquis crassiopibus, simplicibus tarsis omnibus 5 -articulatis.

Longit. 21/4 lin. Latit. 11/3 lin.

Erichson, Entomographien p. 59. 9.

Mlataciuus mistrio Dejean, Cat. $3^{\text {me }}$ édit. p. 122.

IIabitat in California DD. Eschschollz et Tschernikh.

161. Dasytes haticollis: Oblongis, subcylindricus, supra nigro-reneus, remote punctulatus, parce griseo-pubescens, subtus niger, "capite lato clypeo triangulari, thorace transverso, elytrorum latitudine, elytris dorso subdepressis.

Longit. $11 / 2$ lin. Latit. $2 / 3$ lin.

Habitat in California D. Tschernikh. 
162. Dasytes parvicollis : Elongatus, subcylindricus, supra nigro-æneus, remote subtilius punctulatus, dense griseo-pubescens, subtus niger, capite minore rotundato, thorace elytris angustiore subrotundato, elytris dorso subconvexis.

Longit. 11/4 lin. Latit. $1 / 2$ lin.

Inabitat in California D. Tschernikh.

163. Dasites canescens Eschscholtz: Oblongus, subcylindricus, nigro-æeneus, griseo-pubescens, thoracis medio et lateribus elytrorumque macula utrinque baseos humerum ambiente et fasciis duabus postcrioribus transversis denudatis, antennis basi rufo-testaceis, thorace elytrorum fere latitudine.

Longit. $11 / 2$ lin. Latit. $1 / 3$ lin.

Dejean, Cat. $3^{\text {me }}$ édit. p. 125

IIabitat in California D. Eschscholtz; ad describendum benevole communicavit Excell. D. Fischer de Waldheim.

\section{TEREDILES.}

161. Cuerus exminus: Niger, hirtus, capite thorace, corpore subtus pedibusque dense canopilosis, thorace maculis duabus nigris, elytris nigyro-cyaneis, macula triangulari baseos, fascia media anterius acuminata, margine cum macula basali connexa suturaque posterius coc- 
cmeis, elytrorum apice cano-piloso, medio nigro-maculato.

Longit. 4 lin. Latit. $1 \frac{1}{2} 2$ lin.

Lectus a D. Blaschke in nave c California ad Americam meridionalem iter faciente, ideoque verosimile Californix incola.

165. Corynetes rufipes Fabricius: Viridi-cocrulescens, supra nigro-pilosus, subtus grisen-pubescens, thorace punctulato, elytris striatopunctatis, antennarum basi pedibusque rufis.

Longit. $2 \frac{1}{5}, 2 \frac{1}{2}$, lin. Latit. 1, 11/4, lin.

Fubricius, Systema Eleuther. I. p. 286.2.-Herbst, Natur. syst. aller Ins. IV. p. 151, 2.

Schönher, Synonym. Insect. II. p. 51. 2.-Boisduval, Faune entom. de l'Ocćanie p. 143.-Dejean, Cat. $3^{\text {mo }}$ edit. p. $12 \%$.

Necrobia nufipes Olivier Entomologie IV. 76 bis. p. 5. 2. Tab. 1. fig. 2. a. b.-Latreille IIist. nat. des Crust. et des Ins. IX. p. 156. 2.-Stephens Illustr. of British Entomology. III. p. 327. Manual of British. Coleopt. p. 178.1570 .

VAn. b. Cœrulco-violaceus, elytris subtiliter rugulosis, cactera ut in a.

C. Retrculatus Eschscholtz in litteris.

Habitat in California; VAR. a. a D. Blaschle, VAR. b. a DD. Eschscholtz et Blaschke allata. Occurrit in Europa meridionali, Asia, Africa, America et Nova Hollandia.

166. Anobium paniceun Linné. Ovatum, breve, subcylindricum, ferrugineum, sericeo-pubescens, thorace convexo æquo, elytris tenue 
strictis, punctis minutissimis in striis impressis, inceretitiis subtilissime rugulosis.

\section{Longit. 11/2, 2 lin. Latit. $2 / 5,1$ lin.}

Fabr. Syst. El. I. p. 323. 9.-Oliv. Entom. II. 16. p. 10. 8. T. 2. fig. 9. a, b.-Herbst. Natursyst. $\mathrm{V}$ p. 59. 5. Tab. 47. fig. 6.-Gyllenhal, Ins. suec. 1. P. 293. 5.Schönherr, Syn. Ins, II. p. 105. 15.- Lutreille Hist. natur. des Crust. et des Ins. IX. p. 183. 5.-Sturm, Deutschl. Fauna. XI. p. 135. 18.-Dejean, Cat. $3^{\mathrm{mo}}$ édit. p. 130.-Stephens, Illustr. of British Entom. III. 340. Manual of British Colcopt. p. 201. 1596.

Dermestes paniceus Linné, Fauna suecica p. 145. 431. Systema Naturæ I. II. p. 564. 19.

Habitat in California D. Blaschke; insectum per totam orbem obvium.

16\%. Ptines Fur Linné: Fusco ferrugineus pubescens, thorace quadri-fasciculato, ely tris crenatostriatis interrupte albo-bifascialis, femoribus elongatis, clavatis.

Mas: elytris oblongis subcylindricis.

Femina: elytris gibbosis ovatis.

Longit. $11 / 2$ 2 lin. Latit. $\%, 1$ lin.

Linné Systema Naturx II. p. 560. 5.-Fabr. Syst. El. I. p. 325. 6.-Oliv. Entom. IJ. 17. p. 6. 3. Tab. fig. 1. Latreille. Ilist. nat. des Crust. el des Ins. IX. p. 173. ¿.-Gyllenhal, Ins. succ. 1. p. 307. 5.-Schönherr, Syn. Ins. Ii. p. 107. 5.-Stephens Illustr. of British Entom. 1Ii. 3ง4. intanual of British Colcopt. p. 200. 1581.Eejzar, Ciat. $3^{\text {me }}$ édit. p. 130.

CFmaibix fur Linné, Fauna succ. p. 190. 657. 
VAR. b. testaceus immaculatus.

Ptinus latro Fabr. Syst. El. I. p. 326. 9. (ठ゚)--Oliv. Ent. 11. 17. p. 7. 4. Tab. 1. fig. 3. (오).

Ptinus testaceus Oliv. Ent. II. 17. p. 9. 8. Tab. 2. fig. 9. (रे).

Habitat in insula Sitkha et in California D. Blaschke. Species valde noxia in omnibus terro regionibus vulgata.

\section{CLAVICORNIA.}

168. Necrophorus nigrita: Niger, thorace glabro, basi punctato, elytris supra acqualibus subtiliter punctulatis, seriebus tribus e punctis minutis impressis, antemnarum clava aurantiaca, pectore nigro-piloso, abdomine glabro, tibiis posticis rectis.

Longit. 9 lin. Latit. 4 lin.

Ilabitat in California D. Tschernikh.

N. Hưmaror Gaze similis, elytris subtiliter punctulatis, absque ullis lineis elevatis pectoreque nigro-piloso ab illo vero omnino diversus. N. Lateralis et auropilosus Eschsch.; e California in Catalogo ill. Com. Dejean inscripti, mihi ignoti, sed vix ad speciem jam descriptam referendi.

169. Necrophorus maritimes Eschscholtz: Niger, thorace glabro, antennarum clava elytrorumque fasciis duabus aurantiacis, pectore dense griseo-pubescente, abdomine glabro, tibiis posticis reclis. 
$V_{\text {AR. b. feminæ; fascia elytrorum anteriore }}$ evanescente, loco ejus punctis tantum duobus in utroque elytro, et margine inflexo angustius aurantiaco.

Var. c. utriusque sexus, ut Var. b., sed fascia elytrorum anteriore omnino deficiente et posteriore angustiore.

Longit. 9 lin. Latit. $3^{3} / 4$ lin.

Dejean, Cat. $3^{\text {me }}$ édit. p. 131.

Habitat in insula Sitkha DD. Eschscholtz et Blaschke; Variet. c. Mus. Univ. Imp. Mosqu. specimina tria examinavi.

N. ruspatoni Erichson (vestigatori Gyllenh) similis, sed differt imprimis clytris longioribus, postice latioribus, dorso magis depressis et fasciis angustioribus, abdomine toto glabro.

170. Sirpha cervaria Eschscholtz: Ovata, leviter convexa, nigra, opaca, thorace antice parum emarginato, supra coriaceo, elytris lineis tribus elevatis undulatis, interstitiis lineis confuse ramosis.

VAR, b. elytris obscure virescente-æneis, evidentius granulato-punctatis.

Longit. 6 lin. Latit. $3 \frac{1}{2}$ lin.

Dejean, Cat. $3^{\text {me }}$ édit. p. 132.

llabitat in California DD. Eschscholtz et brlaschle; specimen VAr. a benevolentia D. Obert debeo; VAr. b. in I.us. Univ. Imp. Mosqu. asservatam ad describendum communicavit D. Rowillier. 
Statura et magnitudo S. Reticulate Fabr. thorace convexo coriaceo ei similis, sed thorax in hac specie anterius angustatus apice nonnihil cmarginatus et elytrorum sculptura valde diversa.

171. Silpha califonnica Eschscholtz: Ovata, brevis, depressa, nigra, capite thoraceque griseotomentosis, elytris trilineatis, interstitiis tuberculis globosis, subseriatis.

Femina: elytris apice sinuatis, juxta suturam valde candato-productis.

Mas latet.

Var. b. Luberculis in interstitiis elytrorum longe ante apicem evanidis.

Longit. $4^{3} / 4,5^{1 / 2}$ lin. Latit. 3, 31/2 lin.

Dejean, Cat. $3^{\text {me }}$ édit. p. 132.

Habitat in California DD. Eschscholts et Tschernikh. VAR. b. a D. Dr. Fischer ad Ross capta.

S. caudate Say (turbencolate Germar) affinis, sed brevior, latior, thoracis basi magis sinuato et elytrorum tuberculis rarioribus distincta.

172. Necrophulus Hydropuituoides Eschscholtz: Ovatus, convexus, Jrunneo-piceus, thoracis elytrorumque marginibus dilutioribus, thorace transverso, apice angustato, elytris profunde crenato-striatis.

Longit. 5 lin. Latit. 3 lin.

Dejean, Cat. $3^{\text {me }}$ édit. p. 132.

Ptomophaga HydrornLoddes Eschscholtz in litteris. $N^{\circ}$ II. 1843. 
Perates Escirscuoltzn Fischer de Waldheim in litteris.:

Habitat in insula Sitkha D. Eschscholtz.

N. subterraneo Illiger fere quadruplo major, longior, thorace breviore et variis notis distinctus.

173. Catops Cadaverinus Eschscholtz: Oblongoovatus, fusco-piceus, tenue pubescens, antennis mediocribus, clavatis, basi ferrugineis, thorace brevi transverso, basi parum latiore, angulis posticis obtusis, elytris rufescentibus punctatis, stria suturali impressa, pelibus ferrugineo-piceis, femoribus infuscatis.

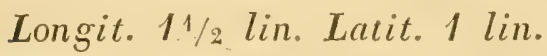

Cators fuscus Hoffmansegg Var. Dejean, Cat. $3^{\text {me édit. }}$ p. 133.

Habitat in insula Sitkha DD. Eschscholtz et Kuprianoff.

C. Morionı Fabr. Erichs. (fusco Gyllenh.) nonnihil affinis, thorace minore, angustiore et elytris longioribus ab illo distinguendus.

174. Carpopillus hemipterus Linné: Oblongo-ovatus, supra nigro-fuscus, pubescens, elytris dimidiatis, macula humerali apiceque late flavis, pedibus ferrugineis.

Longit. $1^{1 / 3}$ lin. Latit. ${ }^{2 / 3}$ lin.

Stephens, Illustr. of British Entom. III. 50. Manual of British Colcopt. p. 122. 974.

Nitidula bimacurata Oliv. Entom. II. 12. p. 6. 5. Tab. 2. fig. II. a. b.-Gyllenhal, Ins. suec. 1. p. 244. 34.-Schönherr, Syn. Ins. II. p, 14\%, 58. 
Nitidula flexuosa paykull, Fauna succ. I. p. 354. 9.IIerbst, Natur-Syst. V. p. 246. 21. Tal. 54. fig. 5.

Dermestes nempterus Linné, Syst. Nat. I. II. p. 565.50.

Irs emacurata Dejean, Cat. $3^{\text {me }}$ édit. p. 134.

Habitat in California D. Plaschke; ex Europa cum rebus mercatoriis verosimile allatus.

175. Strongruus? trictu; : Vigro-fuscus, opacus, creberime punctulatus, griseo-pubescens, thorace convexo, elytris abbreviatis, macula discoidali obsoleta ferrnginea, antemnis pedibusque rufis. $\left(^{*}\right)$

Longit. $21 / 3$ lin. Latit. $11 / 4$ lin.

Habitat in California D. Tschernikh.

176. Nitidula convexiuscula: Ovata, convexa, diluta testacea, punctatissima, thorace antice emarginato, elytris apice singulatim rotundatis.

Longit. $11 / 3$ lin. Latit. $2 / 3$ lin.

Habitat in insula Sitkha DD. Kuprianoff et Blaschke.

N. Depnesse Illig. Gyllenhal minoribus individuis valde affinis, thorace autem paulo angustiore, antice profun-

(*) Der abgekürzten Flügeldecken wegen wäre diese Art wohl eigentlich zu Stroxgrues nicht zu rechnen, allein da ich die Gattungen Brachypepus und Catopterus Erichson eben so wenig als Curzeus Laporte kenne, wage ich nicht darüber zu entscheiden, sondern lasse lieber diese Art mit den ihr verwandten, nach Dejean, unter Strongrues bleiben. 
dius emarginato et elytris apice rotundatis imprimis diversa.

177. Nitidula ambigua: Oblonga, subdepressa, rufo-ferruginea, punctatissima, tenue fulvopubescens, marginibus dilutioribus, thorace antice leviter emarginato, elytris apice singulatim rotundatis.

Longit. $11 / 3$ lin. Latit. $1 / 2$ lin.

Habitat in insula Sitkha D. Eschscholtz. Mus. Univ. Imp. Mosqu.

Media inter N. pygman Gyllenhal et oblongam Herbst, Gyllenhal, illa longior, in elytris magis parallela, hac paulo convesior, utrisque fortius punctulata.

178. Cryptophagus californicus: Oblongo-ovatus, convexus, pallide testaceus, punctatus, pilis flavis pubescens, thorace anterius angustiore, medio transversim impresso, margine laterali acuto serrato.

Longit. 1 lin. Latit. $1 / 2$ lin.

Habitat in California D. Blaschke.

179. Cryptophagus Quadridentatus: Oblongo-ovatus, fusco-ferrugineus, pilis flavis pubescens, capite thoraceque profunde punctatis, thoracis lateribus utrinque acute bidentatis, postice crenulatis, elytris subtilius punctulatis.

Longit. 1, 11/3 lin. Latit. 1/3, 1/2 lin.

Habitat in insula Sitkha D. Blaschke.

C. Lycoperdi Fabr. affinis, sed angustior, antennis crassio- 
ribus, thorace fortius punctato, lateribus acutius dentato, posterius crenulato distinctus.

Variat colore totius corporis pallidius ferrugineo.

180. Dermestes Vulpixus Fabricius: Oblongus, niger, cincreo-subpubescens, subtus niveus, maculis marginalibus denudatis atris, thoracis lateribus densius cinereo-villosis.

Longit. 3, 4 lin. Latit. 1/1/, $1 \overline{3} / 4$ lin.

Fabr. Syst. EI. I. p. 314. 12.-Oliv. Entom. II. 9. p. 8. 4. Tab. I. fig. 6.-Herbst Natur-Syst. IV. p. 12 1. 5. Tab. 40. fig. 5. c.-Schünherr Syn. Ins. Il. p. 89. 21.Gyllenhal, Ins. suec. I. p. 147. 2.-Stephens Illustr. of British Entom. I1I. 123. Manual of British Colcopt. p. 143. 1153.-Dejean, Cat. $3^{\text {me }}$ édit. p. 139.

Habitat in insula Sitkha D. Blaschke; per totum orbem terrarum obvius.

181. Dermestes Lupryes Eschscholtz: Oblongus, piceus, dense fusco-cincreo-tomentosus, subtus niveus, nigro-maculatus, thoracis lateribus densius cinereo-villosis.

Longit. $3^{2 / 3}$ lin. Latit. 12/3 lin.

Dejean, Cat. $3^{\text {me }}$ p. édit. 139.

Habitat in California D. Eschscholtz.

Statura et magnitudine procedentis, sed subtilius punctulatus et pubcscentia multo densiore fusco-cinerea imprimis diversus.

182. Dermestes Talpivus Eschscholtz: Oblongus, niger, supra cincreo-fulvoque marmoratus, scutello dense fulvo-pubescente, subtus ni- 
veus, abdominis margine nigro-punctato, antennis nigris, articulo ultimo rufo-piceo.

Longit. 3 lin. Latit. $1 \frac{1 / 2}{2}$ lin.

D. Catta Panz. Var. Dejean, Cat. $3^{\text {me }}$ édit. p. 139.

IIabitat in California D. Eschscholtz; e Mus. Univ. Imp. Mosqu. a D. Rouillier ad describendum benevole communicatus.

D. MUnı⿰ Linné minor, maculis cincreis elytrorum vix vel perparum undulatis et maculis fulvis immixtis sparsis diversus mihi videtur, cætcrum forma eadem, unde ut varietas ejus facile putandas.

183. Dermestes marmoratus Say: Oblongo-ovatus, niger, thorace pilis fulvis longius pubescente, maculis tribus minutis e pilis cinereis transversim positis, elytris maculis sparsis marmoratis et plaga magna humerali interius angulata e pilis albido-cinereis, subtus niveus, abdominis margine nigro-punctato, antennis rufopiceis.

Longit. $31 / 2$ lin. Latit, $11 / 2$ lin.

Say, Ocurres entom. trad, par Gory p. 159, 1.-Dejean,

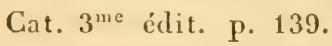

IIabitat in California D. Blaschke; in Missuri et Arkansas Amer, septentr. frequens. D. Say.

184. Antunenus apicalis: Niger, creberrime punctulatus, parce griseo-pubescens, elytrorum apice pedibusque rufescentibus, thorace lato, basi sinuato, medio valde producto.

Longit, 1 lin. Latit. $2 / 3$ lin. 
Habitat in California D. Blaschlie.

185. Saprinus calrfornicus Eschscholtz. Nigroæneus, ruguloso-punctatus, thorace antice foveolato, disco elytrorumque area antica communi lævibus, nitidis, striis quinque obliquis dimidiatis, suturali antice abbreviata, tibiis anticis sex dentatis.

Longit. 21/2, 3 lin. Latit. 2, 21/ lin.

Hister califonnicus Dejean, Cat. $3^{\text {me }}$ édit. p. 142.

Habitat in California D. Eschscholtz; c Museo Univ. Imp. Mosqu. ad describendum bonevole misit D. Rouillier.

186. Saprinus sulcifroxs: Suloquadratus, convexus, nigro-æneus, fronte lineis duabus, posteriore adscendente acuminata, impressis, thorace basi punctulato, elytris creberime rugulosopunctatis, reliclis areolis scutellari, humerali et apicali externa levibus, striis quatuor obliquis inxuralibus, tibiis anticis tridentatis.

Longit. 21/* lin. Latit. $12 / 3$ lin.

Habitat in California D. Eschscholts. Mus. Univ. Imp. Mosqu.

187. Ceutocerus Advexa Schäppel: Subquadratus, convexus, testaccus, nitidus, oculis nigris, thorace emarginato, basi bisinuato, lateribus deplanato, elytris subquadratis abdomen obtegentibus, subtiliter striato-punctatis.

Longit. $1 / 2$ lin. Latit. $1 / 3$ lin.

Germar, Coleopt. Spec. nov. p. 85. 146.-Dejean, Cat. $3^{\text {no }}$ édit. p. 144. 
Habitat in insula Sitkha D. Blaschke; cum rebus mercatoriis certe allatus.

\section{PALPICORNIA.}

*188. Elophorus arricollis Eschscholtz: Capite thoraceque viridi-aureis, punctato-rugosis, thorace angulis acutis, quinque sulcato, sulcis lavibus, elytris griseis, interstitiis striarum alternis carinatis.

Longit. 11/2 lin.

Eschscholtz, Entonographien p. 43. 20.

Habitat in insula Unalaschka D. Eschscholtz.

189. Cercyon lmibum: Ovatum, convexum, nigrum, elytris tenue punctato-striatis, fuscopiceis, thoracis margine anguste, elytrorum late apiceque rufis.

VAr. b. elytris rufo-picens dorso infuscatis.

Var. c. elytris rufo-ferrugineis.

Longit. $1 \frac{1 / 3}{3}$ lin. Latit. ${ }^{5} / 6$ lin.

Habitat in insula Sitkha DD. Kuprianoff et Blaschke.

C. flavipens Fabr. Erichs, simile, sed brevius, fortius punctatum, elytrorum striis profundioribus et colore diversum.

190. Crercyon adumbratuy: Oblongo ovale, convexum, ferrugincum, elytris profunde punctatostriatis, dilutioribus, disco fusco-adumbratis.

Var. b. obscurius, fusco-piceum, capite 
thoraceque nigro-piceis, thoracis margine laterali testaceo, elytris disco latius infuscato.

Longit. 1 lin. Latit. ${ }^{2 / 3} \cdot$ lin.

Habitat in insula Sithka DD. Kuprianoff et Blaschke.

Magnitudine C. atomari Fabro sed longius, minus globosum, elytra minus profunde punctato-striata, et interstitia plana.

\section{LAMELLICORNIA.}

*191. Aphodius aleutus Eschscholtz: Capité trituberculato, ater, thoracis dense punctati angulis, elytris pedibusque rufo-piceis, elytrorum striis punctatis, interstitiis seriebus punctorum subtilissimis.

Longit. $2^{2 / 3}$ lin.

Eschscholtz, Entomographien p. 2\% 11.-Sturm, Cat. p. 95. Habitat in insula Unalaschka D. Eschscholtz.

*192. Aphodius gutratis Eschscholtz: ater, capite quadrituberculato, elytris striis punctatis, maculis quadratis ferrugineis sparsis.

Longit. $2 \frac{1}{2}$ lin.

Eschscholtz, Mém. de la Soc. Imp. des natur. de Moscou VI. p. 9\% 1.-Sturm, Cat. p. 95.

Habitat in fimo bovino insulæ Unalaschkx frequens $D$. Eschscholtz.

193. Oxyouus Cadaverixus Eschscholtz: Oblongus, supra nigro-subtus rufo-piceus, clypeo pro- 
funde emarginato, thorace anterius dilatato varioloso, elytris punctato-striatis.

Longit. 3 lin. Latit. $12 / 3$ lin.

Aphodius Cadaverinus Dejean, Cat. $3^{\text {me }}$ édit. p. 162.

Habitat in California, Trogium instar in cadaveribus exsiccatis D. Eschscholtz.

194. Oxronus crumdricus Eschscholtz: Oblongus, nigro-piccus, clypeo subintegro, transverso, rugoso, thorace crebre punctato, lateribus crenulato, basi sulcato, elytris punctato-striatis, interstitiis planis, punctis minutis subseriatis.

Longit. $2^{1 / 3}$ lin. Latit. 11/ lin.

Dejean, Cat. $3^{\text {me }}$ édit. p. 163.

Psamodies cylindricus Eschscholtz, Entomogr. p. II. 3.

Ilabitat in insula Unalaschka locis elevatis sub lapidibus et in insula Sitkha D. Eschscholtz.

195. Sinodendron rugosua: Nigrum, thorace rugoso-varioloso antice truncato, unidentato, capitis cornu recurvo, parce piloso, elytris valde ruguso-intricatis.

Longit. 5 lin. Latit. 2 lin.

IIabitat in California D. Tschernikh; ab Excell. D. Fischer de Waldheime amicissime communicatum

Statura S. crindoricr, sed undique valde rugosum et thorace unidentato omnino diversum. 


\section{HETEROMERA.}

\section{MELASOMATA}

*196. Nyctoporis cnistata Eschschollz: Nigra, capite cristato, thorace breviore varioloso, medio foveis duabus profundis, elytris cristis granulatis elevatis, humeris spinosis, basi abrupte declivibus.

Longit. 6 $\frac{1}{2}, 7$ lin.

Eschscholtz, Zool. Atlas IV. p. 11. Tab, 18. fig. 4.Dejean, Cat. $3^{\text {me }}$ édit. p. 203.

Habitat in California, in littore meridionale Sinus St. Franzisco. D, Eschescholtz.

197. Nyctoponis xeuicollis Eschscholtz: Nigropicea, capite thoraceque quadrato varioiopunctatis; inæqualibus, elytris cristis granulatis subtilibus, humeris obtusis.

Longit. $6 \frac{1}{2}, 7$ lin.

Eschscholtz, Zool. Atias IV. p. 12.-Dejean, Cat, $3^{\mathrm{ma}}$ édit. p. 203.

Ifabitat in California, in littore septentrionali Sinus St. Franzisco D. Eschscholtz.

198. Eulabis bicaruxata Eschschollz: Oblonga, picea, thorace ruguloso-punctato, medio bicarinato, lateribus reflexis, subangulatis, clytris planatis costatis, interstitiis profunde striatopunctatis, pedibus rufis.

Longit. $32 / 3,4$ lin Latit. $1 \frac{1 / 2}{2} 12 / 5 \mathrm{lin}$. 
Eschscholtz, Zool. Atlas. III. p. 15. 1. Tab. 14. fig. 8.Dejean, Cat. $3^{\text {me }}$ édit. p 203.

IIabitat in California ad St. Franzisco locis e littore maris distantibus. DD. Eschscholtz ct Tschernikh.

199. Eulabis Rufipes Eschscholtz: Oblonga, picea, clypeo, antennis, thoracis lateribus pedibusque rufis, thorace profunde aciculato, xquali, lateribus rotundato, tenue marginato, elytris convexiusculis costatis, interstitis profunde striato-punclatis.

Longit. 2, 4 lin. Latit. 1, 11/2 lin.

Eschscholtz, Zool. Atlas. III. p. 15. 2.-Dejean, Cat. $3^{\text {me }}$ édit. p. 203.

Habitat in California ad St. Franzisco in vicinate Missionis, D. Eschscholtz.

*200. Eurymetopon rufipes Eschscholtz: Semicylindricum, atrum, antennis ferrugineis, pedibus rufo-piceis, thorace basi apiceque truncato, lateribus rotundato, elytris posterius declivibus, striato-punctulatis, striis remotis basi extrorsum curvatis, interstitiis internis irregulariter, externis suberiatim punctulatis.

Longit. $3^{1 / 2}$ lin.

Eschscholtz, Zool. Atlas. IV. p. 8. Tab. 18. fig. 1.

IIabitat in California ad St. Franzisco sub lapidibus D. Eschscholtz.

*201. Euryetopon ochraceun Eschscholtz: Oblongum, pallide ochraceum, capite paulo obscuriore, thorace subcylindrico, elytris medio 
gibbosis posterius declivibus, subtiliter striatopunctatis, punctis basi et apice evanescentibus, interstitiis remote punctulatis, punctis subseriatis.

Longit. $1 \frac{1 / 4}{\text { lin. }}$

Eschscholtz, Zool. Atlas. IV. p. 8. Tab. 18. fig. 2.

Habitat in California ad St. Franzisco sub lapidibus D. Eschscholtz.

202. Apocrypha Antulcordes Eschscholtz: Oblonga, convexa, rufo-ferruginea, elytrorum dorso nigro, undique subtiliter punctulata, capite elytrisque pilis rarioribus, thorace densioribus, griseo-pubescentibus, thorace suborbiculato, antice truncato, elytris ovalibus.

Var. b. Fusca, antennis pedibusque rufopiceis, elytris macula utrinque humerali rufoferruginea.

Longit. $1 \frac{1}{2}$ lin. Latit. $1 / 2$ lin.

Eschscholtz, Zool. Atlas. IV. p. 13. T'ab. 18. fig. \%

Habitat in California ad ripas fluviorum D. Eschscholtz.

VAR. b. a D. Blaschke captam amicissime dedit Excell.

D. Lange.

203. Amphidora littoralis Eschscholtz: Oblonga, nigro-fusca, pilis longis raris fuscis villosa, thorace transverso, convexo, latitudine elytrorum, lateribus rotundato, antice posticeque truncato, crebre punctato, elytris dense 


\section{6}

striato-punctatis, inierstitiis angustis, seriatim subtilissime punctulatis.

Longit. $3 \frac{1}{2}, 4$ lin. Latit. $1 \frac{1}{2}, 12 / 3$ lin. Eschscholtz, Zool. Atlas. IV. p. 13. Tab. 18. fig. 6.-Dejean, Cat. $3^{\text {ne }}$ édit. p. 231.

Habitat in California locis planis ad littora maris, inter herbas et sub lapidibus D. Eschscholtz.

204. Eleodes sulcipennis: Elongata, nigra, opaca, capite profunde punctato, thorace subcordato, lateribus tenue reflexo-marginato, subtiliter punctulato, elytris oblongis, profunde sulcatis, postice perparum dilatatis, apice attenuatis, valde declivibus, femoribus anticis dentatis.

Longit. $142 / 5$ lin. Latit. 5 lin.

IIabitat in California Excell. D. Wrangel. Mus. Imp. Acad. Scient. Petrop.

205. Eleodes grandicollis: Elongata, nigra, supra convexa nitida, capite profunde punctato, thorace lateribus valde rotundato-ampliato, reflexo-marginato, subtiliter punctulato, elytris oblongis, versus apicem modice angustatis, levissime rugulosis, obsolete striato-punctatis, femoribus anticis dentatis.

Longit. $14^{2} / 3$ lin. Latit. $41 / 2$ lin.

Habitat in California DD. Wrangel et Tschernikh; specimen e Mlus. Imp. Acad. Scient. Petrop. ad describendum amicissime communicavit D. Ménétriés, altcrum Excell. D. Fischer de Waldheim. 


\section{7}

206. Eleodes gigantea: Elongata, nigra, supra nitida, thorace rotundato, supra convexo, basi apiceque latitudine xquali, femoribus muticis, elytris subtiliter granulato-punctatis, maris versus apicem sensim attenuatis, feminæ sesqui latioribus, pone medium dilatatis apice subito angustatis.

Mas: Longit. 151/2 lin. Latit. $4^{1 / 3}$ lin.

Femina: Longit. 16 lin. Latit. $5^{1 / 2}$ lin.

Habitat in California Excell. D. Wrangel. Mus. Imp. Acad. Scient. Petrop.

Gigas in hoc genere, statura sequentis nonnihil similis, multo major et forma magis elongata.

207. Eleodes dentipes Eschscholtz: Elongata, nigra, supra nitida, capite thoraceque subtiliter granulato-punctatis, thorace lateribus rotundato, basi coarctato, angulis anticis acuminatis, elytris subtiliter striato-punctatis, punctis granuliferis, femoribus anticis dentatis.

Mas: angustior, elytris pone medium sensim attenuatis.

Longit. 8, 10 lin. Latit. 3, 31/2 lin.

Femina: latior elytris medio paulo dilatatis, versus apicem declivibus, minus angustatis.

Longit. 10, 12 lin. Latit. $4 \quad 4^{1 / 2}$ lin.

Eschscholtz, Zool. Atlas. III. p. 10. 1. Tab. 14. fig. 4.- 
Guérin, Magas. de Zoologie. IV. p. 31. pl. 117. fig. 1.-Dejean, Cat. $3^{\text {me }}$ édit. p. 209.

Habitat in California ad St. Franzisco, locis elevatis DD. Eschscholta, Blaschke et Tschernikh.

208. Eleodes Quadricolits Eschscholtz: Oblonga, nigra, capite fortius, thorace subtilius punctulato, subquadrato, antice paulo latiore, modice rotundato, femoribus muticis.

Mas: subcylindricus, elytris thoracis fere latitudine pone medium angustatis declivibus, dorso dense striato-punctatis punctis granuliferis.

\section{Longit. 7 lin. Latit. $2 \frac{1}{2}$ lin.}

Femina: lata, obovata, elytris thorace fere duplo latioribus, mox pone humeros dilatatorotundatis, pone medium rotundato-angustatis, declivibus, dorso rugulosis, subtiliter granulatis, obsolete striato-punctatis.

Longit. $9^{1 / 2}$ lin. Latit. $4^{2} / 3$ lin.

Eschscholtz, Zool. Atlas. III. p. 12. 9. Tab. 14. fig. 5.Dejean, Cat. $3^{\mathrm{me}}$ édit. p. 209.

Habitat in California ad St. Franzisco, locis elevatis frequens DD. Eschscholtz.

209. Eleodes marginata Eschscholtz: Oblonga, nigra, thorace transverso, medio crebre punctato, lateribus rotundato, granulis nitidis scabro, marginibus reflexis, elytris ovatis pone nedium angustatis, granulis reclinatis scabris, 


\section{9}

femoribus anticis maris dentatis, feminx muticis.

Longit. 7, $7^{2} / 3$ lin. Latit. 31/2, $3^{2 / 3}$ lin.

Eschscholtz, Zool. Atlas. III. p. 10. 2.-Dejean, Cat. $3^{\mathrm{mm}}$ édit. p. 210.

Habitat in California ad St. Franzisco rarius DD. Esch. scholt $\mathrm{z}$ et Tschernikk.

210. Eleodes Fischeri. Oblonga, niggra, thorace transverso, lato, medio punctato, lateribus valde rotundatis, tenue marginatis, granulis nitidis scabriusculis, elytris ovalibus, apice attenuatis, tuberculis piliferis subseriatis reclinatis dense asperatis, femoribus anticis muticis.

\section{Longit. 9 lin. Latit. 4 lin.}

Mannerheim, Revue Zoolog. par la Société Curiéricune 1840. १. $13 \%$.

IIabitat in California ad Ross. D. D. ${ }^{\mathrm{r}}$ Fischer.

Prxcedenti affinis, thorace hreviore, latiore, minus crebre punctato elytrisque longioribus magis rugosis et scabris ut et femoribus anticis muticis diversa.

211. Eleodes claviconnis Eschscholtz: Ovata, nigra, thorace lateribus rotundato tenne. marginato, ibique et in medio punctato, area utrinque lavi, postice truncato angulis minutis rectis, elytris posterius sensim attenuatis, dorso nonnihil deplanatis; foveolis subquadratis seriatis impressis, lateribus granulatis et pilo$N^{\circ}$ II. 1843. 
sis, antennis articulis tribus ultimis precedentibus duplo latioribus.

Longit. $5 \frac{1}{2}$ lin. Latit. $2^{2 / 3}$ lin.

Eschscholtz, Zool. Atlas III. p. 11. 3.-Dejean, Cat. $3^{\text {me }}$ édit. p: 210.

Habitat in California, locis planis ad littora maris D. Eschscholtz.

Specimina e Mus. Univ. Imp. Mosqu. ad examinandum benevole misit D. Rouillier.

212. Fifedes reflexicollis: Ovata, nigra, thorace brevi, lato, subdepresso, Jasi subito-angustato, lateribus valde rolundatis, reflexo-marginatis, dorso crebre punctato, areis duabus laevigatis, elytris convexis, punctatis, punctis tuberculis minutis nilidis instructis, humeris obtusis, antemis extrorsum vix crasioribus.

Longit. 6 lin. Latit. 3 lin.

Ilabitat in California ad Ross. D. D. Fischer.

Sequenti proxima, ejusdem magnitudine et statura, scd thorax latior, profundius punctatus, lateribus magis rotundatus, margine altius reflexo basique magis ab. rupte angustatus et elytra crebrius punctato-tuberculata, minime at in illa costulis elevatis substriata.

2) 13. Fieodes planata Eschscholtz: Oblongo-ovata, nigra, thorace transverso, basi coarctato, lateribus valde rotundalis, tenue rellexo-marginatis, dorso dense punctato, areolis duabus lacvigatis vix distinctis, elytris dorso depressis, seriatim foveolato punctatis, obsolcte subco- 
statis, humeris obtusis, antennis "subfiliformibus.

Longit. 6, 74/2 lin. Latit. $2^{3 / 4}, 31 \frac{1}{4}$ lin.

Eschscholtz, Zool. Atlas III. p. 12. 6.-Dejean, Cat. $3^{\text {me }}$ édit. p. 210.

Habilat in California ad Koss frequens D. Eschschollz.

214. Eleodes parvicollis Eschscholtz: Ovata, nigra, thorace brevi transverso, lateribus rotundato, tenue reflexo-marginato, basi subito coarctato ibique latitudine apicis angustiore, dorso profunde punctato, lateribus dense granulifero, elytris dorso punctis majoribus subseriatis leviter impressis, lateribus granulatis, humeris subrectis margine nonnilil reflexo, antennis filiformibus.

Longit. $5 \frac{1}{2}$ lin. Latit. 3 lin.

Eschscholtz, Zool. $\Lambda$ tlas. III. p. 11. 4.

Habitat in California ad St. Franziseo rarius D. Eschscholtz et Bluschke.

215. Er.rodes producta: Oblonga, nigra, thorace lateribus rotundato, tenue marginato, basi angustato, ibique et apice latitudine æquali, dorso crebre punctato, lateribus dense granulifero, elytris oblongo-ovatis, pone medium attenuatis, dorso Jeviter punclatis, punctis granuliferis, versus latera asperatis, humeris rotundatis antrorsum nonnihil productis, antennis extrorsum paulo incrassatis.

Longit. 6 lin. Latit. $2 \frac{1}{2}$ lin. 
Habitat in California D. Blaschke.

Præcedenti affinis, sed multo longior et angustior, thorace longiore et latiore, elytrisque undique asperatis, subrugosis imprimis diversa.

216. Eleodes scabrosa Eschscholtz: Oblonggo-ovata, nigra, thorace transverso punctatissimo, basi apiceque latitudine equali, lateribus valde rotundato, granulis scabro, elytris ovatis dorso rude punctatis, subrugosis, lateribus granulatis asperis, humeris subrectis, antennis articulis tribus ultimis latioribus.

Mas: elytris latitudine thoracis.

Femina: elytris thorace fere sesqui latioribus.

Longit. 5, 51/2 lin. Latit. $2 \frac{1}{4}, 3$ lin.

Eschscholtz, Zool. Atlas. III. p. 11. 5.-Dejean, Cat. $3^{\text {me }}$ édit. p. 210.

Habitat in California ad St. Franzisco frequens DD. Eschscholtz et 'T'schernikh.

217. Eleodes cordata Eschscholtz: Ovata, nigra, thorace transverso, sub-convexo, lateribus valde ampliato-rotundatis, basi sinuatim coarctato, angulis subprominulis, undique tenue marginato, fortiter et crebre rugoso-punctato, elytris apice declivibus, dense punctato-rugosis et granulatis, lateribus tuberculis reclinatis valde exasperatis, humeris prominulis subrotundatis, antennis extrorsum leviter incrassatis. 


\section{3}

Mas: antennis crassioribus, pedibus fortioribus, longioribus.

Femina: antennis et pedibus brevioribus, tenuioribus.

Longit. 5, 7 lin. Latit. 21/2, 3 lin.

Eschscholtz, Zool. Atlas. III. p. 12. 7. Tab. 14. fig. 6.Dejcan, Cat. $3^{\text {me }}$ édit. p. 110.

Habitat in California ad St. Franzisco, locis elevatis sat lrequens DD. Eschscholtz et Tschernikh; etiam ad Ross a D. Fischer lecta.

218. Eleodes intricata: Nigra, thorace transverso, lateribus valde rotundato ibique tenue marginato, basi subito coarctato, dorso profunde punctato, elytris convexis apice declivibus, tuberculis lævigatis minutis densissimis rugoso-intricatis, humeris subrotundatis, antennis extrorsum parum crassioribus.

Mas: oblongo-ovata, elytris oblongis, thoracis fere latitudine antennis pedibusque longioribus et fortioribus.

Longit. 5 lin. Latit. 2 lin.

Femina: ovata, mare sesqui latior, elytris thorace latioribus, antennis pedibusque tenuioribus.

Longit. 5 lin. Latit. 3 lin.

IIabitat in California ad Ross. D. D. ${ }^{\mathrm{r}}$ Fischer.

Sequenti affinis, thorace latiore, basi subito angustato, punctato, haud rugoso, elytris punctis asperatis multa minoribus, densioribus omnino distincta. 
219. Eleodes ruberculata Eschscholtz: Oblongoovata, nigra, thorace subconvexo, corlato, lateribus rotundato, tenue reflexo-marginato, basi coarctato, angulis rectis, supra rude et profunde rugoso-punctato, elytris convexis, tuberculis levigatis reclinatis densis rugosoasperatis, humeris rotundatis, antennarum articulis $5-8$ suborbiculatis, tribus ultimis paullo crassioribus.

$$
\text { Longit. 5, 51/2 lin. Latit. 21/4, } 2^{2 / 3} \text { lin. }
$$

Eschscholtz, Zool. Atlas. III. p. 12. 8..-Dejean, Cat. $3^{\text {me }}$ édit. p. 210.

Habitat in California ad Cabo de los Reyes D. Eschscholtz; a DD. Blaschke et Tschernikh etiam allata.

220. Eleodes Pineliomes: Brevis, nigra, opaca, capite, thoraceque lateribus rotundato crebre rugoso-punctatis, elytris subquadratis, apice valde declivibus, dorso deplanatis, crebre granulatis, granulis reclinatis asperis piliferis.

Longit. 6 lin. Latit. 3 lin.

Habitat in California Excell. D. Wrangel. Nus. Imp. Acad. Scient. Petrop.

Magnitudo E. ranvicoliss, sed statura brevi lata, elytris subquadratis a ceteris congeneribus diversa.

221. Coniontis viatica Eschscholtz: Oblonga, semicylindrica, convexa, nigra, opaca, thorace apice emarginato, angulis deflexis, lateribus tenue marginato, basi subtruucato, angulis productis acutis, dor'so creberrime subtilissime 
punctulato, elytris thorace paulo angustioribus, tenuc marginatis, apice rotundatis, subtilissime parcius punctulatis.

Mas: differt antennis, ore et pedibus brunneo-piceis.

Longit. 6, 71/2 lin. Latit. 3, 31/2 lin.

Eschscholtz, Zool. Atlas. 11I. p. 7 1. Tab. 14. fig. 3.-Dejean, Cat. $3^{\text {me }}$ édit. p. 211.

Habitat in California ad Sinum St. Franzisco, in campis et viis argillaceis a D. Eschscholtz capti.

222. Conrontis Eschscholtzir: Oblonga, semicylindrica, convexa, nigra subnitida, thorace apice cmarginato, angulis deflexis, antice et lateribus tenue marginato, basi subtruncato, dorso subtilissime minus crebre punctulato, elytris thorace paulo angustioribus, tenue marginatis, dorso, prasertim posterius, valde punctato-rugosis, pilis albis brevissimis obsitis.

Nas: minor, brevior, minus convexus, thorace latiore angulis posticis subrectis, lateribus parum sinuato, tibiis tarsisque anticis nonnihil dilatatis.

Femina: major, valde convexa, thoracis lateribus posterius sinuatis, angulis posticis productis rotundatis, tibiis tarsisque anticis mediocribus.

Longit. 6, 7 lin. Latit. 3, 31/2 lin.

Mannerheim, Revue Zoolog. par la Soc. Cuvier. 1840. p. 138. 


\section{6}

IIabitat in California ad Ross. D. D. Fischer; specimen a D. Tschernikh missum etiam communicavit Excell. D. Fischer de Waldheim.

223. Coniontis nemoralis Eschscholtz: Oblonga, convexa, nigra, subnitida, thorace breviore transverso, apice emarginato angulis vix deflexis, lateribus tenue maryinato, basi subtruncato angulis productis acutis, dorso subtiliter crebre punctulato, elytris thoracis laticudine, tenue marginatis, versus apicem rotundato-angustatis, dorso profunde rugosopunctatis, pilis brevissimis albidis obsitis.

Mas: elytris mox pone humeros versus apicem sensim angustatis, tibiis tarsisque anticis nonnihil dilatatis.

Femina: elytris pone medium versus apicem angustatis magis declivibus, tibiis tarsisque anticis mediocribus.

Variat antennis, pedibus et elytrorum apice brumneo-piceis.

Longit. $3^{1 / 2}, 6$ lin. Latit. $13 / 4,3$ lin. Eschscholtz, Zoolog,. Atlas III. p. 8. 2.

C. campestris ? Dejean, Cat. p. 212.

IIabitat in California ad St. Franzisco locis arenosis inter arbusta D. Eschscholtz; etiam ad Ross a D. Fischer capta.

224. Blapstinus pulverulentus Eschscholtz: Oblongus, ater, antennis, pedibusque piceis, creberrime punctatus, thoracis angulis posticis 
subrectis, elytris punctato-striatis, striis versus suturam profundius impressis.

Longit. 2, 3 lin. Latit. $8 / 9,11 / 3$ lin.

Dejean, Cat. $3^{\text {me }}$ édit. p. 213.

Habitat in California DD. Eschscholtz, Blaschke et Tschernikh.

\section{TAXICORNES.}

225. Coelus cilratus Eschscholtz: Rotundatoovatus, convexus, brunneo-piceus, antennis brevissimis pedibusque rufo-piceis, thorace transverso, apice late emarginato, basi truncato, lateribus rotundato, ibique et apice tenue marginato, dorso subtiliter punctulato, elytris granulis scabris, corporis margine pedibusque pilis longis ferrugineis ciliatis.

Lungit. 3, 31/2 lin. Latit. $12 / 3,2$ lin.

Eschscholtz, Zool. Atlas. III. p. 5. Tab. 14. fig. 1.Dejean, Cat. $3^{\text {me }}$ édit. p. 215.

Habitat in California ad St. Franzisco, locis arenosis sub stercoratis exsiccatis D. Eschscholtz.

226. Phaleria picta Eschscholtz: Brevis, subhemisphærica, punctulata, pallide rufo-ferruginea, thoracis basi utrinque bistriato, elytris punctato-striatis, in utroque macula media dentata nigro-fusca.

Longit. $1 \frac{1}{2}$ lin. Latit. 1 lin.

Dejean, Cat. $3^{\text {me }}$ édiț. p. 216. 
Ilabitat in insula Sitkha D. Eschscholtz. Mus. Univ. Imp. Mosqu.

227. Stene ferrucinea Fabricius: Limeari-elongata, depressa, rufo-ferruginea, thorace transverse quadrato, tenue marginato, confertissime subtiliterque punctulato, elytris tenue punctato-striatis, interstitiis punctulatis, antennarum articulis tribus ultimis majoribus perfoliatis.

Longit. $11 / 3,2$ lin. Latit. $1 / 2,2 / 3$ lin.

Stephens, Illustr. of British Entom. V. 9. Manual of British Coleopt. p. 322. 2531.

Margus ferruglineus Dejean, Cat. $3^{\text {me }}$ édit. p. 222.

Trogosita ferruginea Fabr. Syst. El, 1. p. 155. 23. Eit. Syst. I. p. 116. 7.

Tenebrio ferrugineus Fabr. Mant. I 212. 1\% Spec. Ins. 1. 324. 12. - Oliv. Ent. III. 5\% p. 18. 25. Tab. 2. fig. 24. a. b.

Colydeum castaneum Herbst, Natur-Syst. ViI. p. 282. 3. Tab. 112. fig. 3.

Tenebrio testaceus Schönherr Syn. Ins. I. p. 153. 46.

Pinalenia castanea Gyllenhal, Ins. succ. II. p. 588, 2.

Habitat in insula Sitkha D. Blaschke; per totum orbem terrarum obvia, animalia in Musæis asservata, panem aliaque cibaria destrucns. 


\section{TENERRIONIDES.}

\section{GENTRIOPTERA.}

Antennæ moniliformes, articulis 1 et 2 brevissimis, 3 elongato, subcylindrico, $4-6$ hoc triplo brevioribus, sulyquadratis, $7-9$ lenticularibus rotundatis compressis, 10 brevi transversa subcyathiformi, ultimo parvo rotundato, apice acuminato.

Labrum exsertum, apice rotundatum, ciliatum.

Mandibulæ corneæ, validæ, intus unidentatæ, apice incurvo emarginato.

Maxillæe coriaceæ, extus membranacex, fasciculato-ciliatæ, muticæ.

Mentum corneum, tricarinatum, apice emarginatum.

Labium exsertum, profunde emarginatum, ciliatum.

Palpi omnes articulo ultimo præcedente parum longiore subobconico, apice truncato.

Corpus oblongum, apterum, statura fere Carabi.

Caput clypeo rotundato, jugulo crebre ruguloso, spatio medio triangulari rugis majoribus transversis instructo.

Pedes validi, femoribus versus apicem nonnihil incrassatis, interne profunde excavatis, 


\section{0}

tibiis pilis brevibus rigidis exasperatis, apice bispinis, quatuor anterioribus perparum incurvis, posticis retrorsum nonnihil curvatis, tarsis articulis compressis, convexis, 1-4 utrinque spinula armatis, unguiculis validis incurvis, muticis.

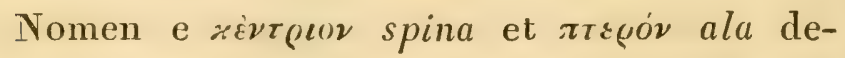
rivatum.

228. Centrioptera Carabordes: Nigra, nitida, capite profunde parce punctato, thorace elytris latiore, subcordato, anguste reflexo-marginato, subtilissime sparsim punctulato, elytris profunde striato-punctatis, lateribus posterius trifariamı spinulosis.

Longit. 13 lin. Latit. 4 lin.

IIabitat in California Excell. D. Wrangel. Mus. Inp. Acad. Scient. Petrop.

\section{COELOCNEMIS.}

Antennæe extrorsum leviter incrassata, articulis 1 et 2 breviusculis, 8 elongato subcylindrico, 4-7 hoc fere duplo brevioribus extrorsum paulo incrassatis, 8 et 9 lenticularibus compressis, latere interiore nomnihil productis, subemarginatis, denticulo minuto subarmatis, 10 et 11 lenticularibus compressis rotundatis, mulicis, ultimo perparum majore. 
Labrum coriaceum, exsertum, transversum, apice subemarginatum.

Mandibulæ validx, corneæ, apice emarginatæ.

Maxillæ coriaceæ, apice bifidæ.

Mentum corneum, leviter bicarinatum, apice rotundatum.

Labium subexsertum, integrum, margine ciliato.

Palpi maxillares, articulo ultimo, magno, brevi, lato, oblique truncato, labiales articulo ultimo majore obconico, apice truncato.

Corpus subelongatum, apterum. Caput supra planum, clypeo truncato, sub-emarginato, lateribus ante oculos ampliato, ibique nomnihil elevato, lobo utrinque oculis superposito, jugulo transversim crebre rugoso.

Pedes clongati, femoribus versus apicem nonnihil incrassatis, interne profunde excavatis, tibiis prasertim anterioribus incurvis, omnibus spinulis duabus brevissimis terminatis, latere interno extrorsum profunde canaliculatis, in quatuor posterioribus canalicula lateribus dense spongioso-setosa, tarsis articulis subcompressis, subtus dense spongiosis, articulo ultimo longiore, unguiculis incurvis muticis.

Nomen e roiru: carus et xrịu tibia compositum. 
229. Coelocnemis californica: Nigra, subnitida, crebre punctulata, thorace cordato, undique tenue marginato, elytris oblongis, tenue striato-punctatis, in singulo seriebus novem e punctis oblongis impressis.

$$
\text { Longit. } 7^{1 / 3} \text { lin. Latit. } 2 \frac{1}{2} \text { lin. }
$$

Habitat in California D. Blaschke; a D. Obert ad describendum amice communicata.

230. Coelocnemis dinaticollis: Convexa, nigra, subnitida, subtiliter punctulata thorace subcordato, lateribus valde rotundato-dilatato, tenue marginato, elytris oblongo-ovatis, pone medium dilatatis, apice declivibus angustatis, in singulo seriebus acto striato-punctatis.

Longit. $10^{1 / 2}$ lin. Latit. $4 \frac{1}{2} 2$ lin.

Ilabitat in California D. Eschscholtz; e Mus. Imp. Univ. Mosqu. ad describendum benevole misit Rouillier.

\section{V. CIBDELIS $\left({ }^{*}\right)$.}

Antennæ extrorsum leviter incrassátæ, articulis 1 et 2 breviusculis, 3 elongato, subcylindrico, 4-7 sensim brevioribus extororsum

${ }^{*}$ ) Diese drei neuen Gattungen machen, der verwachsenen Flügeldecken wegen, cinen Uebergang zu der Familic der Melasomen, allein die Form des Kopfes mit den Fresswerkzcugen und der Beine weisen ihmen wieder cincu Platz unter den T'enebrioniden in der Nähe von Upis an. 
nomnihil incrassatis, 8-10 lenticularibus compressis, apice leviter cmarginatis, ultimo precedente plus quam duplo longiore, oblique ovato, apice subacuminato.

Labrum subexsertum, transversum, apice subtruncatum.

Mandibulæ validx, cornex, apice emarginatx.

Mentum corneum, rugosum, medio subcarinatum, apice rotundatum, basi constrictum.

Maxillæ coriacex, apice obtusæ, longe ciliate.

Labium exsertum, apice integrum, subrotundatum, longe ciliatum paraglossis liciniatis.

Palpi omnes articulo ultimo magno brevi lato, subsecuriformi.

Corpus oblongum, apterum, elytris connatis. Caput supra planum, clypeo lato, apice leviter rotundato, lateribus ante oculos nonnihil elevato, jugulo leviter transversim striguloso.

Pedes mediocres, femoribus versus apicem haud crassioribus, tibiis anticis vix incurvis, omnibus spinulis duabus brevissimis terminatis, apice ipso dense spongioso-setosis, tarsis articulis subcompressis, subus dense spongiosis, articulo ultimo elongato, unguiculis incurvis muticis.

Nomen a $x_{i} \beta \delta \eta_{\eta} \lambda i ;$ spurius. 
231. Cibdelis Blaschin: Nigra, thorace convexo, subquadrato, punctato-ruguloso, lateribus integro, modice rotundato, undique tenuc marginato, elytris pone medium versus apicem angustato-rotundatis, humeris antrorsum productis, dorso subtiliter striato-punctatis, interstitiis tuberculis nitidis seriatim dispositis.

Longit. $6^{1 / 2}$ lin. Latit. 3 lin.

Ilabitat in California DD. Blilschke et Tschernikh.

232. Nyctobates serrata Eschscholtz: Elongata, nigra, opaca, crebre punctulata, thorace cordato, subdepresso, lateribus inæqualiter serrato, elytris posterius dilatatis, subtiliter seriatim punctatis, humeris rotundatis, tibiis omnibus rectis.

Longit. 10 lin. Latit. 4 lin.

Iputunus serratus Dejean, Cat. $3^{\text {me }}$ édit. p. 225.

Upis serrata Eschscholtz in litteris.

Habitat in California ad St Franzisco D. Eschscholtz.

233. Nyctobates inermis Eschscholtz: Elongata, nigra, subnitida, capite thoraceque profunde punctatis, hoc subquadrato, anterius vix angustato, undique tenue marginato, elytris subtiliter punctulatis, striato-punctatis, posterius nomihil dilatatis, humeris oblique subrotundatis, tibiis leviter incurvis.

$$
\text { Longit. } 10^{\frac{1}{2} 2} \text { lin. Latit. } 4 \text { lin. }
$$

Uprs iveras Eschscholtz in litteris. 
I'utunus Sipendoinfs Var. Dejean, Cat. $3^{\text {mo }}$ édit. p. 225

Habitat in California ad St. Franzisco. D. Eschscholts.

N. Sapendoun Busc vilde affinis, sed diversa mihi videtur, capite thoraceque multo fortius punctatis, thorace latio. re, anterius vix angustato, et elytris longioribus, parcins punctulatis.

2:34. Prtho? Sahlbergir: Elongatus, valde convexus, rufo-ferrgineus, capite thoraceque obscurioribus profunde punctatis, illo fronte transversim valde impressa, hoc brevi, subcordato, immarginato, basi apiceque truncato, lateribus anterius rotundato, elytris foveolis oblongis profundis crebre punctatis, apice lavigatis (*).

Femina major, in elytris multo latior et pedibus brevioribus tenuioribus diversa.

Mas: Longit. $3^{2} / 3,4^{1 / 3}$ lin. Latit. $11 / 4$, $2 \frac{1}{2} \operatorname{lin}$.

(*) Dieser Käfer ist wahrscheinlich der, als auf Sitkha vorkommend, von Eschscholtz (Rcise um die Welt in den Jahren 1823-26 von Kotzebue, $2^{\text {ter }}$ Th. Uebersicht der zoologischen Ausbeute von $F_{r}$. Eschscholtz, p. 26.) erwähnte Buros. Seinc stack gewölbte Gestalt macht ihn freilich einen Boros täuschend ähnlich; allein bei genaucrer Untersuchung findet man, dass die Fühlhörner. und Fusswerkzeuge mit denen von Рутно ganz übereinstimmen, nur ausgenommen dass das letzte Glied der Antennen nicht so scharf zuge. spitzt ist. Die convexe Form wärc wohl nicht eine wohlge. gründete Auleitung ihn von der letztgenannten Gattung zu tresnen. 
Femina: Longit. $1^{2 / 3}$ lin. Latit. $1^{2 / 3}$ lin. IIabitat in insula Sitkha DD. Eschscholtz, Kuprianoff et Tschernikh

\section{HELOPI.}

\section{EUCYPHUS.}

Antenne monitiformes, articulo primo longiore curvato, 2 et 3 paulo brevioribus xqualibus obconicis, 4-10 præcedentibus nomnihil brevioribus, inter se xqualibus subquadratis, compressis, ultimo paulo majore ovato apice rotundato.

Labrum exsertum, transversum, antice subemarginatum, ibique margine triangulariter incrassatum.

Palpi maxillares labialibus multo longiores, articulo ultimo mayno securiformi; eodem labialium etiam majoris magnitudinis, triangulari, apice truncato.

Mandibulæe trigonæ, depress

Mentum parvum, apice emarginatum.

Corpus globosum, forma fere Hroson vel Agialex, antice posticeque inflexum. Caput sub thorace retractum, illoque angustius, rotundatum, oculis magnis oblongis. Thorax transversus, anterius angustatus. Scutellum conspicuum. Elytra valde convexa, gibba. pedes breves, tibiis ommibus latiusculis, fulvo- 


\section{7}

hirsulis, anticis latere interiore excavatis, medio in angulum dilatatis, quatuor posterioribus extrorsum profunde excavatis, extus in medio rotundato-dilatatis, ibique crebre spinulosis, tarsis breviusculis, subtus hirsutis, articulo antepenultimo subtus membrana oblonga instructo, unguiculis incurvis integris.

Nomen ab $\varepsilon v$ valde et xuqris gibbus derivatur.

235. Eucyphus Hyosonoides: Supra piceo-æneus, glaber, nitidus, creberrime punctatus, subtus brunneo-piceus, antennis tarsisque rufo-ferrugineis.

Longit. $3 \frac{1}{3}$ lin. Latit. 2 lin.

Habitat in California D. Blaschke.

236. Helops californicus Eschscholtz: Oblongus, fusco-æneus, capite thoraceque subquadrato creberrime punctatis, elytris striatis, in striis et interstitiis subtiliter punctulatis, antemis pedibusque piceis.

Mas: antennis capite cum thorace longiores, extrorsum crassiores。

Femina: antennis capite cum thorace breviores, filiformes.

Longit. 4 lin. Latit. $11 / 2$ lin.

Dejean, Cat. $3^{\mathrm{me}}$ edit, P. $33 \mathrm{f}$.

IIahitat in California DD. Eschescholts et Tschernikh. 


\section{8}

\section{TRACHELIDES.}

23\%. Anaspis SERICEA. Elongata, brunneo-picea, supra holosericeo-pubescens, antennarum basi, ore elytris pedibusque ferrugineo-testaceis, fulvo-sericeis.

Longit. $12 / 3$ lin. Latit. $2 / 3$ lin.

$V_{A R}$. b. thoracis margine antico, angulisque posticis ferrugineis.

Ilabitat in insula Sitkha D. Blaschke. VAR. b. in Museo Acad. Imp. Petropol. asservatur.

238. Anaspis pallescens Eschscholtz: Elongata, fulvo-ferruginea, subtilissime griseo-pubescens, antennis extrorsum, oculis abdomineque nigro-fuscis.

Longit. $12 / 3$ lin. Latit. $3 / 4$ lin.

Dejean, Cat. $3^{\text {me }}$ édit. p. 242.

Hahitat in insula Sitkha D. Eschscholls. Mus. Univ. Imp. Mosqu.

Pracedente paullo latior, prasertim in thorace, colore, pubescentia multo minus condensata, antennisque extrorsum crassioribns distincta.

\section{VESICANTIA.}

239. Epicauta puncticollis: Atra, pubescens, capite thoraceque canaliculato, postice impresso, crebre et profunde punctatis, elytris creberrime rugoso-punctatis.

Longit. $4 \frac{1}{2}$ lin. Latit. $1 \frac{1}{2}$ lin. 
Ilabitat in California DD. Mlaschke et T'schernikh; specimina descripta benevole communicaverunt DD. Fischer de. Waldhcim et Obert. Е. Атпоте Fabr. nonnihil similis, sed multo profundius punctata.

\section{TETRAMERA.}

\section{CURCULIONIDES.}

240. Apion cuprescens: Oblongum, fusco-æneum, griseo-pubescens, rostro longitudine thoracis cum capite, arcuato, thorace anterius angustato, profunde punctato, postice canaliculato, elytris oblongo-ovatis, punctato sulcatis , punctis in sulcis satis approximatis.

Longit. cum rostro $1 \frac{1}{2}$ lin. Latit. $1 / 2$ lin.

Habitat in insula Sitkha D. Blaschke; specimen a D. Obert amicissime donatum.

241. Apion troglodytes Schönherr: Breve, nigrum, longius griseo-pubescens, rostro capite cum thorace breviore, parum arcuato, thorace subcylindrico, antrorsum vix angustato, profunde et concinne foveolato-punctato, elytris ovatis, gibbis, sulcatis, sulcis remote punctatis. Longit. cum rostro $3 / 4$ lin. Latit. $3 / 8$ lin. Habitat in California D. Blaschke.

Ad Stirpis $2^{\text {dae }}$, Manipuli $1^{\text {mi }}$ Centuriam $1^{\text {mam }}$ operis Cel Schönherr pertinent ambx.

*2) 12. Sitones californicus Eschscholtz: Oblongus, niger fuscocinereo-tomentosus et setulosus, 
fronte canaliculata; rostro subcostato; thorace evidenter punctato, griseo-trivittato, antemnis tibiisque anticis fusco-ferrugineis, oculis subdepressis.

Schönherr, Genera et Spec. Curcul. VI. p. 26\% 28.

Strona caujfornicus Dejean, Cat. $3^{\text {me }}$ édit. p. 278.

Habitat in California Do. Eschscholtz.

Magnitudo S. 8-punctatr (op. Schönhl.), paulo tamen latior; S. ordinario (ejus. op.) habitu coloreque valde propin. quus, dimidio autem minor; fronte haud retusa, to. mentoque setuloso precipue distinctus.

243. Sitones seniculus: Oblongus, subcylindricus, niger, cinereo-tomentosus et setosus, fronte canaliculata, rostro excavato, thorace profunde rugoso-punctato, lateribus vix rotundato, elytris profunde punctato-striatis, antennarum basi tibiisque ferrugineis.

Longit. $12 / 3$ lin, Latit. $1 / 2$ lin.

Ilabitat in California D. Tschernikh.

244. Alophus alternitus Say: Oblongo-ovatus, niger, fusco-cupreo-squamosus, thorace crebre ruguloso, dorso carinula parum elevata, abbreviata instructo, utrinque obsolete albidolineato, elytris subtiliter punctato-striatis, nigro et pallido tessellatis, macula postica subargentea ornatis, interstitiis confertim granulatis.

Schönherr, Gener. et Spec. Curcul. 11. p. 286. 2. 
Curculio almanatus $S a$ j. in litteris.

VAR. b. elytris etiam macula laterali ante medium obliqua niveo-squamosa.

Longit. cum rostro 6 lin. Latit. $2 \frac{1}{2} \mathrm{lin}$.

Habitat VAR, b. in insula Sitkha D. Blaschke. VAR. a. Amer. septentr. incola.

245. Lixus poricolus Eschscholtz: Oblongus, niger, pube grisea dense obtectus, rostro breviore recto, carinato, thorace antice parum angustiore, dorso punctis nigris remotis variolosis impresso, hasi foveolato, longitudinaliter anguste carinato, utrinque nigro-lineato, elytris subtiliter punctato-striatis, apice singulatim subacummatis, fusco-trilineatis, femoribus muticis.

Longit. cum rostro 4,5 lin. Latit. 11/4, $11 / 2$ lin.

Dejeūn, Cat. $3^{\text {me }}$ édit p. 295.

IIabitat in California. D. Eschscholtz; Mus. Univ. Imp. Mosqu.

Ad Stirpis $2^{\text {dae }}$ Hanipulam $1^{\text {mum }}$ Operis Cel. Schönherr adnumerandus.

246. Lixus moвestus Dejean: Elongatus, niger, griseo-pubescens, rostro crassiusculo, modice arcuato, thorace conico breviore, dorso Jongritudinaliter excavato, elytris striato-punctatis, dense cinereo-squamalosis, maculis minutis albescentibus remote adspersis, apice singulatim subacuminatis, femoribus muticis 
Longit. cum rostro 5 lin. Latit. $1 \frac{1 / 3}{\mathrm{l}}$ lin. Dejean, Cat. $3^{\text {me }}$ édit. p. 296.

Lixús adspersus Eschscholts in litteris.

Habitat in California D. Eschscholts. Mus. Imp. Univ. Mosqu. Stirpis $2^{\text {dao }}$ Manipulo $1^{\text {mo }}$ Operis Cel. Schinhlierr collocandus.

247. Heilipus scrobiculatus: Oblongus, ater, opacus, squalidus, undique inæqualis, scrobiculatus et verrucosus, antennis tarsisque piceis, fronte foveola impressa, rostro parum arcuato, thorace latitudine longiore, lateribus rotundatis, crenatis, dorso carinato, elytris granulis minutis adspersis, verrucis majoribus hine inde immixtis.

Longit. sine rostro 5 lin. Latit. 2 lin.

IIabitat in California D. Eschscholtz; e. Mus. Univ. Imp. Mosqu. ad describendum benevole communicavit D. Rouillier. In Stirpe $1^{\text {ma }}$ Operis Schönherrii inserendus.

218. Anthononus brunnipennis: Nigro-picens, tenuiter pubescens, thorace subconico, profunde rugoso-punctato, scutello cinereo-squamuloso, elytris punctato-striatis, brunneis, antennarum basi, tibiis tarsisqne ferrugineis, femoribus anticis obsolete dentatis.

Longit. sine rostro 2 lin. Latit. 1 lin. Habitat in California D. Tschernikh.

*249. 'Trachodes Ptinomes Eschscholla: Femoribus muticis, picco-niger, thorace oblongo, cari- 


\section{3}

nato, elytris punctato striatis, hispidis, griseoirroratis.

Germar, Col. Spec. nov. p. 32\%, 466.-Schünherr, Gen. et Spec. Curcul. III. p. 513.1.

Pissodes ptinicoluis Sturm. Cat. p. 184.

IIabitat in insula Unalaschka D. Eschscholts.

Magnitudo fere Ermanis ácriduli op. Schünherr.

250. Centrinus confusus Say: Oblongus, ater, sub-nitidus, rostro longitudine thoracis, tenui, valde arcuato, thorace antice angustato, confertim subtiliter punctato, dorso linea angusta, lævi, notato, elytris striatis, striis in fundo punctulatis, interstitiis angustis, crebre striato-punctulatis.

Mas: thorace subtus spinis duabus, parallelis, arcuatis armato.

Femina: thorace subtus mutico.

Schünherr, Gen. et Spec, Curcul. IiI. p. 740, 6.

Ruyncuenus confusus Say in litteris.

Baris sulcipennis Eschscholtz, Dejean, Cat. $3^{\text {me }}$ édit. p 312. Habitat in California D. Eschscholtz; in Florida a D. Say lectus.

Habitus fere Baridi et B. picrio op. Schönherr, nonnihil minor, presertim angustior

251. Sphenophorus discolor Eschscholtz: Oblongoovatus, niger, glaber, rostro crebre punctato, basi tumido, apice compresso, thorace oblongo disperse punctato, tenue canaliculato, elytris punctato-striatis, striis profunde exa- 


\section{4}

ratis, interstitiis remote punctulatis, thoracis lateribus, elytrorum apice, corpore subtus pedibusque extus albidis.

Longit. sine rostro $7^{1 / 2}$ lin. Latit. 3 lin.

Ciandara discolor, Dejean, Cat. $3^{\text {me }}$ édit. p. 329.

Habitat in California D. Eschscholtz; c Mus. Univ. Imp. Mosqu. communicavit D. Rouillier.

252. Sphexophorus subcarinatus: Elongatus, niger, glaber, rostro canaliculato, basi tumido, thorace varioloso-punctato, plagis tribus longitudinalibus elevatioribus, elytris opacis, anguste striatis et in striis punctis minutis remotis impressis, interstitiis impunctatis, suturali lævissimo nitido.

Longit. sine rostro 4 lin. Latit. $11 / 2$ lin.

Habitat in California D. Eschscholtz. Mus, Univ. Imp. Mosqu.

S. confoso Dejean, Schönh. affiuis, sed longior, angustior.

253. Sitophilus Oryze Linné: Oblongus, nigropiceus, opacus, rostro mediocri, striatim punctato, thorace depresso, elytris sub-breviore, confertissime profundius punctato ; elytris confertim creuato-striatis, maculis quatuor rufescentibus notatis.

Mas: rostro crassiore, evidentius carinato, profunde striatim punctato.

Femina: rostro tenuiore, basi tantum cari- 


\section{5}

nato, subtilissime 'striation punctato, extrorsum lovi nitido.

Longit. cum rostro $11 / 3,1 \frac{1}{2}$ lin. Lalit. $1 / 3,1 / 2$ lin.

Schönh. Gen. et Spec. Gurcul. IV. p. 981, 13.

Curculio. Oryzer Linné. Syts. nat. I. IV. p. 1755. 192.

Catanda Orfze Fabr. Syst. El. 1I. p. 438. 4 .

Oliv. Entom. V. 83. p. 97. 34. - Gurculio Tab. 7. fig. 81. a. b.

Slephens Illustr. of British Entom. IV. p. 9. 3.

Manual of British Coleopt. p. 216. 1671.

Habitat in insula Sitkha D. Blaschke; in omnibus terræ regionibus inter grana Oryza obvius.

254. Cossonus Piniphilus Eschscholtz: Nigro-piceus, glaber, supra parum convexus; rostro breviori, crassiori, apice modice dilatato, basi obsolete canaliculato, minus profunde punctato; thorace oblongo, remote sat profunde punctato, anterius angustato, basi leviter bisinuato et obsolete bis impresso, in impressione rude sat crebre punctato; elytris planiusculis profunde punctato-striatis.

Longit, cum rostro 3 lin. Latit. $3 / 4$ lin.

Schönherr, Gen. et Spec. Curcul. IV. p. 1002. 8.-Dejean, Cat. $3^{\text {me }}$ édit. p. 330.

IIabitat in California D. Eschscholtz.

255. Rhyncolus brunneus Eschscholtz. Subcylindricus, rufo-piceus, glaber, antennis mediocribus, rostro longitudine capitis, crebre pun- 


\section{6}

ctato, canaliculato, thorace oblongo, anterius paulo angustato, profunde punctato, elytris profunde punctato-striatis.

Longit. 2 lin. Latil. $1 / 2$ lin.

Dejean, Cat. $3^{\text {me }}$ édit. p. 330.

IIabitat in insula Sitkha DD. Eschscholtz, Kupriunooff et Tschernikh.

\section{XYLOPHAGI.}

256. Hylurgus sericeus Eschscholtz. Brevis, rufopiceus, griseo-sericeus, thorace carinato, elytris squamulis, brumneis cinereisque variegatis, antennis pedibusque rufis.

Longit. $11 / 3,1 \frac{1}{2}$ lin. Latit. $1 / 2,3 / 5$ lin.

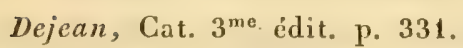

Habitat in insula Sitkha DD. Eschscholtz et Blaschlic.

257. Iylungus obesus Eschscholtz: Brevis, nigropiceus, cinereo-pubescens, antennis, palpis tarsisque rufis, elytris punctato-striatis, interstitiis tuberculato-rugulosis, thorace creberrime punctato, longitudinaliter carinato, ante apicem impresso-constricto.

$$
\text { Longit. } 2^{3 / 4} \text { lin. Latit. } 1 \frac{1 / 3}{3} \text { lin. }
$$

Dejean, Cat. $3^{\text {me }}$ édit. p. 331.

Habitat in insula Sitkha D. Eschscholts. Mus. Univ. Imp. Mosqu.

H. nigniperde Fabr: affinis, sed duplo minor, punctura 
crebriore et subtiliore et thorace breviore, anterius magis angustato, constricto diversus.

258. Hylurgus rugipennis . Oblongus, subcylindricus, supra rufo-piceus, subtus nigro-fuscus, subtiliter cinereo-pubescens, thorace creberrime punctulato, carinato, anterius angustato, elytris profunde punctato-striatis, interstitiis fortiter tuberculato-rugosis.

Longit. 2, $2^{1 / 3}$ lin. Latit. $2 / 3,3 / 4$ lin.

Habitat in insula Sitkha D. Eschscholtz. Mus. Univ. Imp. Mosqu.

H. pallato Gyllenhal similis, sed major, Iongior.

259. Hylurgus pumus: Elongatus, ferrugineotestaceus, capite thoracisque apice obscurioribus, thorace oblongo, crebre punctato, haud carinato, elytris profunde punctato-striatis.

Longit $3 / 4$ lin. Latit. $1 / 5$ lin.

IIabitat in insula Sitkha DD. Kuprianoff et Blaschke.

260. Bostrichus cavifroxs: Cylindricus, nigropiceus, antennis, pedibus thorace subgloboso et elytris apice integris ferrugineo-testaceis, thoracis lateribus antrorsum latius, elytrorumque subtilissime punctato-striatorum margine, sutura viltaque media nigro-fuscis, capite maris profunde excavato.

Longit. $12 / 3$ lin. Latit. ${ }^{3 / 4}$ lin.

Habitat in insula Sitkha D. Eschsicholts. Mus, Univ. Imp. Mosqu. 


\section{8}

B. Liveato Oliv. similis, sed angustior, capite profundius excavato elytrisque subtilius punctato-striatis diversus.

261. Bostriches septentrionis Leconte: Oblongus, brunneus, subnitidus, longius griseo-pilosus , thorace ovato, crebre punctato, obsolete carinato, elytris integris, profunde striato-punctatis, interstitiis seriato-punctatis.

Longit. $12 / 3,2$ lin. Latit. ${ }^{2 / 3}, 3 / 1$ lin.

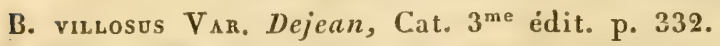

Habitat in insula Sitkha D. Kuprianoff.

B. viuLoso Gyllenhal certe valde affinis, sed longius griseopubescens, profundius punctatus et thorace medio evidenter carinato diversus mihi videtur.

262. Bostrichus terminalis: Oblongus, crebre et profunde punctatus, nigro-piceus, nilis parvis erectis cinereis hispidus, thoracis elytrorumque apice rufis, elytris integris, antennis pedibusque rufo-ferrugineis.

Longit. ${ }^{3 / 4}$ lin. Latit. $1 / 5$ lin.

Habitat in California D. Elaschke.

263. Bostrichus nitidulus: Elongatus, nigro-piceus, nitidus, antennis tarsisque testaceis, thorace profunde punctato, antice asperato, slytris profunde striato-punctatis, apice subretusis, profunde canaliculatis.

Longit. $3 / 4,1$ lin. Latit. $3 / 8,1 / 2$ lin.

Habitat in insula Sitkha D. Blaschke. 
264. Crs viturus: Oblongus, fusco-piceus, punctulatus, pube brevissima rigida adspersus, capite excavato, margine antico quadridentato, thorace apice cornubus duobus antrorsum porrectis armato, antennis pedibusque rufotestaceis.

Longit. $11 / 3$ lin. Latit. ${ }^{2 / 3}$ lin.

Halitat in . California D. Tschernikh.

265. Ladridius Quadricollis: Elongatus, depressus, glaber, ferrugineo-testaceus, oculis nigris , thorace oblongo-quadrato, obsolete canaliculato, medio utrinque foveolato, basi transversim impresso, angulis anticis lobato-productis, elytris oblongis, profunde striato-punctatis, apice obtusis

Longit. $3 / 4,11 / 4$ lin. Latit. $5 / 12,1 / 2$ lin.

Habitat in insula Sitkha D. Blasclrke.

L. axgusticontr Gyllenhal affinis, sed major, prosertim in clytris multo angustiar.

266. Latrimius protensicollis: Elongatus, depressus, glaber, nigro-piceus, antemnis, pedibus elytrisque ferrugineo-testaceis, his macula communi media nigrescente, thorace angustiore, oblongo-quadrato, canaliculato, medio utringue foveolato, basi transversim impresso, angulis anticis lobato-productis, elytris profunde striato-punctatis, apice obtusis.

Longit. $\overline{3} / 4$ lin. Latit. $1 / 2$ lin. 
IIabitat in insula Sitkha D. Blaschke.

Pracedenti aflinis, thorace angustiore, lateribus magis elevato-marginatis, elytris brevioribus et colore diversus.

267. Latridius cordicollis: Elongatus, depressus, glaber, ferrugineo-testaceus, capite subrotundato, thorace cordato, apice truncato, angulis rotundatis, basi transversim impresso, elytris oblongis, profunde striato-punctulatis, apice rotundatis.

Longit. $1 / 2$ lin. Latit. ${ }^{1 / 6}$ lin.

Habitat in insula Sitkha D. Blaschke.

268. Rhyzopingus dimidiatus Eschscholtz: Linearis, subdepressus, nitidus, thorace oblongoquadrato, subtiliter remote punctulato, elytris piceus, capite antice, elytrorum basi, antennis pedibus abdomineque rufo-ferrugineis

Var. b. Corpore toto ferrugineo-lestaceo.

Longit. $11 / 2$ lin. Latit. $1 / 2$ lin.

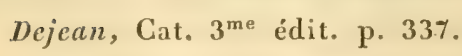

Ilabitat in insula Sitkha D. Eschscholts. Mus. Univ. Imp. Mosqu.

269. Ruagodera tuberculata Eschscholtz: Elongata, sordide nigro-fusca opaca, clypeo magno dilatato, explanato, thorace dorso bicostato, lateribus serrato, elytris conjunctim novemcostatis, interstitiis bifariam profunde striatopunctatis.

Longit. $3 \%$ lin. Latit. I lin. 


\section{1}

Dejean, Cat. $3^{\text {me }}$ édit. p. $33 \%$.

Ilabitat in California D. Eschscholtz; e Mus. Univ. Imp. Mosqu. ad describendum benevole misit D. Rouillier.

270. Silvanus sexdentatus Fabricius: Elongatus, fusco-testaccus pubescens, thorace sulcato, utrinque sexdentato, elytris punctato-striatis, interstitiis alternis elevatioribus

Longit. 1/4 lin. Latit. $1 / 3$ lin.

Gyllenhal, Ins. Suec. III. p. 406. 2.-Dejean; Cat. $3^{\text {me }}$ édit. p. 338.

Dermestes 6-dentatus Fabr. Syst. El. I. 317. 25.

Colydium 6-dentatum Paykull. Fauna Suec. Hil. p. 318. 2.

Colydum frumentarium Fabr. Syst. El. 11. p. 55\% 11.

Ips fruasentaria Oliv. Entom. II. 18. p. 10. 14. Tab. 2. fig. 13. a. b.

Silyanus Surinamensis Stephens Illustr. of British Entom. III. p. 104. Manual of British Coleopt. p. 12\% 1032.

Habitat in insula Sitkha D. Blaschke; in granis Oryza certe allatus.

271. Trocosita culorodia: Elongata, supra viridianea, fronte canaliculata corporeque subtus cyanescentibus, capite thoraceque punctatis, hoc basin versus sinuato-angustato, elytris striato-punctatis, interstitiis seriato-punctatis.

Longit. $7^{\frac{1}{2} / 2}$ lin. Latit. $2 \frac{1}{3}$ lin.

In nave e California ad Americam meridionalem iter faciente a D. Blaschlie capta; verosimile Californix incola.

T. virescresti valde affinis et forsan ejus varietas major.

$\Lambda^{\circ}$ II. 1843.

20 


\section{2}

272. 'Trogosita viridicyanea: Elongata, supracyanea, virili-micans, subtus virescens, fronte canaliculata, capite thoraceque crebrius punctatis, hoc versus basin rotundato-angustato, elytris subtiliter striato-punctatis, interstitiis seriatopunctatis.

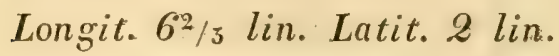

IIabitat in California D. Tschernikh.

A præcedente differt colore, eapite thoraceque crebrius punctatis, elytris paullo brevioribus, subtilius striatopunctatis et thorace versus basin haud sinuato-angustato.

273. 'Trogosita Mauritanica Linné: Elongata, depressa, supra nigra nitida, subtus rufo-picea, thorace cordato, elytris a thorace remotis, tenue punctato-striatis, interstitiis serie duplici punctatis.

Var. b. rufo-ferruginea.

Longit. 3, 4 lin. Latit. $1 \frac{1}{3}, 1 \frac{1}{2}$ lin.

Oliv. Entom. II. 19. p 6. 2. Tab. 1. fig. 2. a. b.-Gyllenhal, Ins. suec. I. p. 72. 1.-Stephens Illustr. of British Entom. IV. p. 224. Manual of British. Coleopt. p. 141. 1139.

Trogosita Caraboides Fabr. Syst. El. I. p. 151.6-Schünherr, Syn. Ins. p. I. 155. 5. -Dejean, Cat. $3^{\text {me }}$ édit. p. 339.

Tenebrio Mauritanicus Linné Syst. Nat. I. II. p. 674. 4.

Habitat in insula Sitkha in cibariis et pellibus D. Blasch$k e$; per totum orbem terrarum obvia. 
2\%. Trogosita pusillina: Elongata depressa, pallide rufo-testacea, thorace basi transversim impresso, lineolis tribus exarato, in medio foveolato, elytris subtiliter crebre punctulatis, stria suturali profunde impressa.

Longit. $1 / 2$ lin. Latit. $1 / 6$ lin.

Habitat in insula Sitkha D. Blaschke.

275. Cucusus puniceus Eschscholtz: Elongatus, depressus, læte sanguineus, antennis nigrofuscis, pectore abdomineque rufo-ferrugineis, thorace subrotundato, lateribus leviter denticulato, supra obsolete bisulcato.

Longit. $6^{1 / 2}$ lin. Latit. $1^{3 / 4}$ lin.

Dejean, Cat. $3^{\text {me }}$ édit. p. 340

Habitat in insula Sitkha D. Eschscholtz; e Mus. Univ. Imp. Mosqu, benevole communicavit Excell. D. Fischer de Waldheim.

C. Depresso Fabr. longior, angustior, colore et capitis thoracisque structura diversus.C. CLAvipede Oliv. adhuc multo longior et ab illo colore, thorace rotundato et capite postice minus producto distinctus.

276. Læmophloeus longicornis: Oblongus, planus, rufo-testaceus, subtiliter punctulatus et pubescens, thorace transverso mutico, apice tenue marginato, basi angustato, supra tenue bistriato, elytris ferrugineis utrinque tristriatis.

Mas: Antennæ corpore multo longiores, articulis $1-4$ brevibus subobconicis, $5-10$ 
antecedentibus fere duplo longioribus subcylindricis, ultimo albuc paulo longiore, apice rotundato.

Femina: Antennæ longitudine capitis cum thorace, moniliformes, articulis subrotundatis, duobus primis et tribus ultimis majoribus.

Longit. $2 / 3$ lin. Latit. ${ }^{1 / 3}$ lin.

Ilabitat in insula Sitkha D. Blaschke.

L. testage Fabr. minor, latior et antennis maris longioribus tenuioribus diversus.

\section{LONGICORNIA.}

277. Spondylis Uifformis Eschscholtz: Elongatus, niger, crebre rugoso-punctatus, thorace subcordato, elytris lineis tribus elevatis ad apicem fere extensis.

Longit. $6^{1 / 2}$ lin. Latit. $2^{1 / 1}$ lin.

Dejean, Cat. $3^{\text {me }}$ èdit. p. 341.

Habitat in insula Sitkha D. Eschscholta; e Mus. Univ. Imp. Mosqu. ad describendum bencvole misit D Rouillier.

Minoribus S. Buprestoidis individuis adhuc minor: angustior, thoracis forma et punctura omnium partium alia distinguendus.

*278. Asemum atrum Eschscholtz: Atrum, thorace punctulato, elytris lineis elevatis obsoletis.

Eschscholtz, Bullet. de la Soc. Imp. des natur. de Moscon II. p. 66 .

Ilahitat in California D. Eschischoltz. 
279. Opsimus quadrilineatus Eschschollz. Elongatus, brunneo-testaceus, subtilissime punctulatus, pube cinereo-flavescente obductus, capite et thorace linea media, elytrisque in utroque lineis binis longitudinalibus, elevatis glabris.

Mas: Antennis corpore interdum longioribus, paullo crassioribus, elytris thorace plus quam triplo longioribus.

Femina: Antennis corpore multo brevioribus, tenuioribus, elytris thorace quadruplo longioribus.

Longit. $4 \frac{1}{2}, 5$ lin. Latit. $1 \frac{1}{2}, 12 / 3$ lin.

Dejean, Cat. $3^{\text {me }}$ édit. p. 354 .

Ilabitat in insula Sitkha DD. Eschscholtz, Kupprianoff, Fischer, Blaschke et Tschernikh; individuum in California captum etiam misit D. Tschernikh, verosimile navibus istuc allatum.

280. Chytus nautscus: Nigro-picens, obscurus, supra parcius, subtus dense cinereo-pubescens, thorace rugoso-punctạto, lateribus et lineis duabus longitudinalibus, elytris macula fasciisque duabus flexuosis cinereo-pubescentibus, his basi, margine suturaque testaceis.

Longit. 7 lin Latit. $2^{1 / 3}$ lin.

Lectus a D. Blaschke in nave e California iter faciente ideoque ut illius regionis incola putandus. 


\section{6}

\section{CHR YSOMELIN Æ.}

281. Donacia Germari Eschscholtz: Viridi-cuprea, antennis nigris, articulis exterioribus basi pedibusque rufis, thorace oblongo-quadrato subtilissime punctulato, antice binodulo, elytris crenato-striatis, interstitiis transversim rugulosis, femoribus posticis unidentatis.

Var. b. fusco-ænea, elytris levius striatopunctatis, subtilius transversion rugulosis, segmentis ventralibus late ferrugineis.

Longit. 31/2 lin. Latit. 11/2 lin

Dejean, Cat. $3^{\text {me }}$ édit. p. 383.

Habitat in insula Sitkha D. Eschscholtz. VAr. b. in Musca Univ. Imp. Mosqu, asservata.

282. Donacia flavipennis Eschscholtz: Ferrugineotestacea, capite antennisque nigris, thorace oblongo-quadrato, posterius angustato, rufo, antice binodulo, elytris crenato-striatis, interstitiis transversim rugulosis, femoribus posticis unidentatis.

Longit. $3^{1 / 3}$ lin. Latit. $1 \frac{1 / 4}{\text { lin. }}$

Dejean, Cat. $3^{\text {me }}$ édit. p. 383 .

Habitat in insula Sitkla D. Eschscholtz; e Mus. Unir. Imp. Mosqu. ad describenđum benevole communicavit Excell. D. Fischer de Waldheim.

Statura ferc precedentis, sed multo angustior et aliter coloratus, elytris profundius striato-punctatis punctis inter se remotioribus etiam diversa. 


\section{7}

283. Syneta carinata Eschscholtz: Flongata, ferrugineo-testacea, ore thoracis elytrorumque marginibus pallidioribus, thorace lateribus unidentato, elytris costis utrinque binis argute elevatis.

Longit. $4^{1} / 4$ lin. Latit. $1 \frac{1}{2}$ lin.

Dejean, Cat. $3^{\text {me }}$ édit. p. 385 .

Ilabitat in insula Sitkha D. Eschscholtz; e Mus. Unir. Imp. Mosqu. ab Excell. D. Fischer de Waldheim missa.

284. Odontota rubrolineata: Oblonga, capite thoraceque profunde punctatis nigris, elytris profunde punctato-striatis atro-cyaneis, thorace macula utrinque laterali elytrisque linea longitudinali media rubris, corpore subtus coeruleo.

Longit. 21/4 lin. Latit, 1 lin.

Habitat in California D). Tschernikh.

2S5. Coptocycla aurisplendens Eschscholtz: Subhemisphærica, rufo-ferruginea, margine omni explanato, elytris leviter striato-punctatis, lateribus compressis, utrinque anterius in dorso foveola leviter impressa infuscata, corpore subtus antennisque extrorsum nigris.

Longit. 21/2, $2^{3 / 4}$ lin. Latit. 2, 21/4 lin.

C. Sexpunctata Var. Dejean, Cat. $3^{\text {ine }}$, édit. p. 397.

Hahitat in California D. Eschscholtz.

C. sexponctate Fabr. certe valde affinis, sed diversa. mihi videtur, magnitudine semper minore, margine thoracis 


\section{$30 \mathrm{~S}$}

elytrorumque minns explanato-dilatatis, elytris angulis humeralibns multo minus productis, striis crebre punctatis, foveola tantum unica dorsali levius impressa et corpore subtus fere toto nigro, ano tantum rufescente.

286. Cassida novemaculata: Subovata, rufo-testacea, margine. explanato ferrugineo, in elytris reflexo, elytris profunde striato-punctatis, lateribus vix compressis, thorace maculis tribus, elystrisque utrinque tribus, scutello antennarum apice corporeque subtus cum pedibus nigris:

Longit. $31 / 3$ lin. Latit. $2 \frac{1}{4}$ lin.

IIabitat in California DD. Blaschke et Tschernikh. Color in vivis læte sanguineus auro-splendens.

28\%. Galleruca flavolmbata: Viridi-æenea, cærulescenti-micans, capite, thorace, pedibus elytrorumque margine flavo-testaceis, capite plaga verticali thoraceque maculis tribus virescentibus, tibiis externe tarsisque infuscatis, femoribus interdum nigro-maculatis.

Longit. 31/4 lin. Latit. $1 \frac{1}{2}$ lin.

Habitat in California DD. Blaschke et Tschernikh; a DD. Fischer de Waldheim et Obert communicata. Magnitudo et fere statura G. Camariensis Paykull.

288. Galderuca punctipennis: Subdepressa, nigra, thorace flavo maculis tribus nigris, ore, antemnarum articuli primi apice, geniculis, tibiarumque basi flavis, elytris concinne punctulatis. 


\section{9}

Longit. $3^{1 / 4}$ lin. Latit. 11/2 lin.

II abitat in California D. Tschernikh.

Statura et magnitudo G. Nrmprex, elytris totis nigris, regulariter concinne punctulatis ab illa mox distin. guenda.

289. Diabrotica trivittata: Oblonga, nigra, thorace fulvo, biimpresso, femorum basi elytrisque pallide flavis, his costulatis, interstitiis bifariam striato-punctatis, vitta media lata et sutura nigris.

Longit. 22/3 lin. Latit. $1 \frac{1 / 2}{2}$ lin.

IIabitat in California D. Tschernikh.

D. vitтate Fabr. afinis, paullo major, nigredine suturali latiore, tibiis totis femorumque maxima parte nigris præcipue diversa.

290. Diabrotica duodecimpunctata Fabricizes: Oblonga, flava, capite, antenuis extrorsum, pectore, femoribus extus, tibiis, tarsis elytrorumque maculis in singulo sex per paria dispositis, nigris.

Dejean, Cat. $3^{\text {me }}$ édit. p. 405.

Crioceris 12-punctata Fabr. Syst. El. I. p. 547. 39.-Schünherr, Syn. Ins. II. p. 273. 40.

Galleruca 12 punctata Fabr. Eut. Syst, I. II. p. 15. 12.

Var. b. in omni puncto simillima, sed antennis nigris, articulis tribus primis hasi testaceis et corpore subtus cum pedibus nigro, subtiliter griseo-pubescente. 


\section{0}

Galleruca 11-punctata Eschscholtz in litteris.

Longit. $31 / 3$ lin. Latit. $11 / 2$ lin.

Habitat in California DD. Eschscholtz et Tschernikh;

$V_{A R}$. b. ibi tantum invenitur, anne re vera species distincta?

291. Graptodera plicipennis: Oblonga, convexa, supra coerulea, subtus cyanea, capite rugosopunctato, thoracis apice vix angustiore, thorace disperse, elytris creberrime punctulatis, his costa marginali elevata posterius ambiente ibique ad suturam connexa.

Longit. $2 \frac{1}{2}$ lin. Latit. $1 \frac{1}{14}$ lin.

Habitat in California D. Tschernikh.

Statura G. Janтunæ Illiger, sed in elytris longior et multo subtilius et crebrius punctulata, thorace levius transversim impresso et costa marginali elytrorum distincta. $\left({ }^{*}\right)$

282. Graptodera californica: Oblongo-ovata, convexa, coerulea, capite thoracis apice multo

(") In Dejean's Catalog. p. 412. findet sich als Varietät von Graptodera plicata Klug eine costata Mannerh, ron der Nordwest Küste Amerikas. Ich bekam freilich von dem verstorbenen Professor Stschegloff einige Stücke dieses Käfers, mit einer solchen Angabe des Vaterlandes; allein da der fragliche Käfer dort sehr haüfig vorkommen sollte und nicht von den auf der Insel Sitkha gewesenen Sammler cx professo gefunden worden ist, muss ich jezt dic Angabe des Herrn Stschegloff bezweifelu. 


\section{1}

angustiore, thorace obsoletissime punctulato, linea transversa postica levissime impressa, elytris subtiliter punctulatis.

Longit. 21/3 lin. Latit. 11/4 lin.

Habitat in California ad Ross D. Fischer.

Statura G. oleracee, sed major thorace convexiore, obsoletissime transversim impresso et elytris subtilius punctulatis distincta.

293. Disonycha maritina : Rotundato-ovata, crebre punctulata, nigra, fronte macula, thorace, elytrorum vittis duabus postice connexis anoque flavis.

VAr. b. capilis macula thoraceque fulvocroceis.

Longit. 2 lin. Latit. $1 \frac{1 / 2}{2}$ lin.

Dejean, Cat. $3^{\text {me }}$ édit. p. 414.

Habitat in California, in plantis ad littora maris DD. Eschscholtz et Blaschke.

294. Chlanys conspersa: Nigro-fusca, foveolatopunctata, thoracis medio pulvinato, elytris tuberculis elevatis scabris, sutura, crenata, fronte, thoracisque apice maculis majoribus notatis, thoracis disco, elytris et pedibus guttis minutis luteis conspersis.

Longit. 1 lin. Latit. ${ }^{2 / 3}$ lin.

Habitat in California D. Tschernikh.

295. Pachyorachys signatifrons: Niger, confertim punctatus, elytris extrorsum punctato-striatis, 


\section{2}

capitis macula magna furcata, thoracis margine laterali, elytrorum basali et subhumerali, femorum basi et macula antica flavoferrugineis.

\section{Longit. $11 / 4$ lin. Latit. ${ }^{3} / 4$ lin.}

\section{IIabitat in California D. Tschernikh.}

296. Cryptocephalus cinalconatus: Cupreo-æneus, fronte, ore, antennis, thorace subtus et lateribus pedibusque rufo-ferrugineis, thorace convexo, postice punctis utrinque quatuor impressis, elytris seriato-punctatis.

Longit. 1 lin. Latit. $1 / 2$ lin.

IIabitat in California D. Blaschke, a D. Obert communicatus.

\section{TRIMERA.}

29\%. Hippodanira vittigera: Oblongo-ovata, nigra, minus convexa, macula frontali, thoracis margine antico et laterali lineaque media, elytrisque margine et vitta longitudinali rufo-testaceis.

Longit. $21 / 3$ lin. Latit. $1 \frac{1}{2}$ lin.

IIabitat in California D. Tschernikh.

298. Coccinela californica Eschschollz: IIemispherica, nigra, capite maculis duabus, thorace utrinque macula apicis elytris fue juxta 


\section{3}

scutellum albidis, elytris rufis, macula scutellari suturaque nigris.

Var. b. elytrorum albecline juxta scutellum. deficiente.

Longit. $2^{2 / 3}$ lin. Latit. $2^{1 / 3}$ lin.

Dejean, Cat. $3^{\text {me }}$ édit. p. $45 \%$

Habitat in California DD. Eschscholtz et Blaschke. VAr.

b. a D. Tschernikh captam misit Exccll. D. Fischer de Waldheim.

299. Scymus marginicoldis : Subhemisphæricus, niger, pubescens, ore, antennis thoracisque margine antico et laterali rufo-ferrugineis.

Longit. 1 lin. Latit. ${ }^{3 / 4}$ lin.

Habitat in Californa.:D. Blaschke.

S. parver. Fabr. statura et magnitudinc similis, niger, undique sat dense griseo-pubescens, ore, antennis thoracisque margine antico et laterali tantum rufescentibus.

\section{DIMERA.}

*300. Batrisus albronicus Eschscholtz: Elongatosubcylindricus, convexus, niger, capite majusculo, in vertice tuberculo parum elevato, medio fossula vix perspicua impresso et sulco lavi circumducto, adjectis foveolis posticis duabus rotundatis, thorace trapezordati, in medio lineola brevissina cet utrinque singula, 


\section{4}

integra ante basin cum tribus foveolis conjunctis, his foveolis sulco transverso bisinuato connexis, elytris castaneo-testaceis.

Longit. $15 / 8$ lin.

Aubé in Guérin Magasin de Zoologie. III. p. 496. Dejean, Cat. $3^{\text {me }}$ édit. p. 466.

Habitat in California D. Eschscholtz. 
Stockholm "'e 26 avil 1854.

Monsieuri,

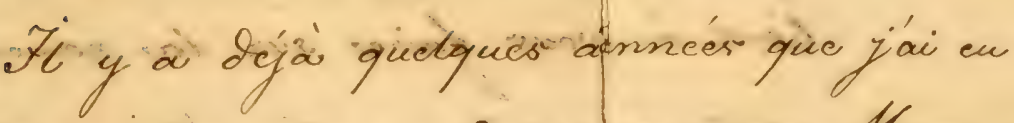
10 jolcisir de recervoir de vorks, ou de Monsicur votre pere un envoi dénsectess, contenant pour La plipart des Carabiques, fies Staphyliniens at des Hesteriens, qui mont beaucoupi interesfe' et dont jei vous remercie bien cordialement. L'envoi était accompagne' d'un catalogue du contenue et de quelques brachures entomologíques. sur les Coarabiques par the Leconte et sur les: Longicornes par Sh. Haideman ourza jes Dont yo suis J'autant plus reconnarifant, que ler production de la littératinge des Ctats. imu four trier-rares et par confequent treiri,recherch in Europe, mais il n'y avait point de lettr. Dans cet envoi. 
- If'aunt dans le temps beaucoup oce Des Ltapyliniens, y aurais en quelques obfer tions à rus faire sur la nomenclature - viotre enoi surmentionne, mais toutes mes. se trountit à Mibourg en finlander, licu ma réfetence ordinaire bien loin D'ici. Hatheurfisicment jes seici ici à ftockhou

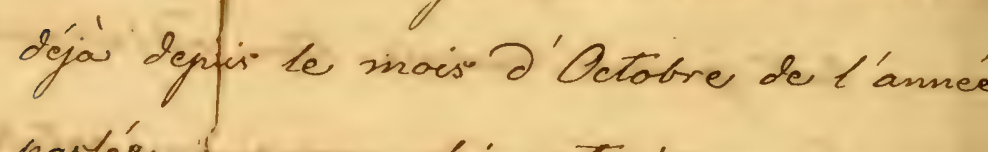
pasfé jur me faires tracter pour une ladies que de plus en plus enleve mes fore de maniejer que ce n'est qu'arec grande peiner quos jé puisfe tracer ces lignes. Q. jó voulu profiter dee depart de c.

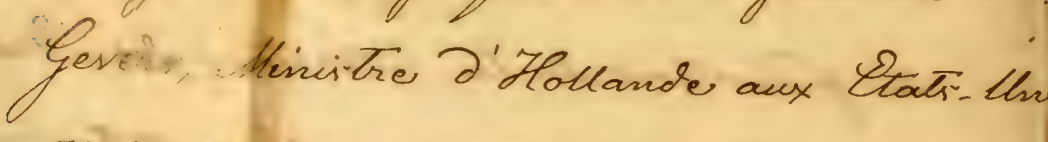
et de raveonzulairance de se charger d'en po quet de spoi, pous vous faire parsenis les fo cond et tipisiéme seyplemens à ma Faune 
coleópterologique des parferfipor Rusfes en A mériques. Le second de ces deux exemplain vour pourrer on le préfentior an Lycée de L'Histoire naturelle de New yorks ow le donn. à quelquím de vos amis entomologister.

Receser, yei vour prie, Alonrieur, les as. furancer de ma confidération la plus des tinguéés. 



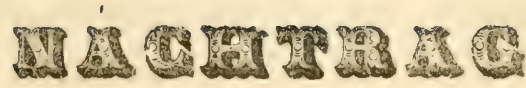

$$
\text { Z U R }
$$

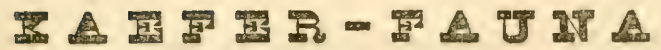

\section{DER ALEUTISCHEN INSELN UND DER INSEL SITKHA}

$$
\text { V } 0 \text { N }
$$

Graf $\mathfrak{C}$. G. Alammerbsiun. 
(Aus d. Bullet. der Naturforsch. Gesellsch. in Moscau, Band 13. 1846.) 


\section{W}

Z $\quad$ U R

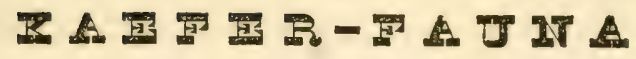

DER ALEUTISCIIEN INSELN UND DER INSEL SITKHA

V O N

Graf C. G. Mannerieim.

Nachdem ich vor drei Jahren eine systematische Aufzählung aller bis dahin auf den Aleutischen Inseln, der Insel Sitkha und in Neu-Californien entdeckten Käfer-Arten herausgab, sind theils von Herrn Ménétriés, Conservator des entomologischen Museums der Kaiserl. Alademie der Wissenschaften, im Bulletin de la Classe Physico-Mathématique gedachter Akademie, Tome II. $\mathrm{N}^{\circ} 4$, theils von Herrn von Motschulsky, Capitain im Kaiserl. Russischen General-Stabe, in diesem Bulletin, Tome XVIII. No I und IV, etliche bisher unbe_ kannt gebliebene Species der Insecten-Ordnung Coleoptera aus denselben Gegenden dem entomologischen Publicum veröfentlicht worden. Wie 
ich es in meiner oben erwithnten fï̈heren $\mathrm{Ab}$ handlung bercits angedeutet habe, beabsichtigte ich wohl eine vollständige Käfer-Fauna der genamnten vom stillen Oceane bewässerten Insel und Küsten-Lünder auszuarbeiten und hatte auch schon das dazu nöthige Material zum grössten Theile in Bereitschaft liegen, allein indem ich erfuhr, dass die Akademie der Wissenschaften Herrn Ménétriés den Auftrag ertheilt, einen Catalog mit Diagnosen der netien Arten, die IIerr Wosnesensty beonders aus Californien heimgeschickt hatte, anzufertigen, entschloss ich mich nur einen Prodromus meincr Käfer-Fanna oder eine diagnosti.iche Auseinandersetzung aller mir bekanuten Arten herauszugeben. Ich wollte dadurch llerrn Ménélriés das Monopol die neuen

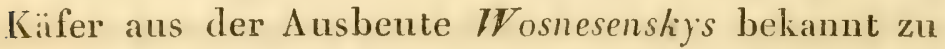
machen unangetastet erhalten und ihm keineswegs die Gelegenheit entnehmen, über diese Entdeckungen in der Käfervelı, ein dem Standpuncte einer Akademie der Wissenschatten entsprechendes vollständiges Werk zu schreiben. Indessen sind doch jezt fast drei ganze Jahre verstrichen, ohne dass wir von Herrn Ménétriés ètwas weiteres als die Bearbeitung der Carabicinen mic 16 von ihm angefertigten Diagnosen vorher unbekannter Species erhalten haben, und es ware also hohe Zeit eine l'ortsetzung dicses entomographischen Unternehmens zu erwarten. Der wohlgegründeten Bemerkung des Herrn Professor 
Erichson (') mich anschliessend, dass die Fanna der Aleuten und Sitkhas so wenig imern $Z_{n}$. sammenhang mit der von Californien hat, weswegen es zweckmässiger gewesen wäre, beide gesondert und in sich abgeschlossen zu behaudeln, überlasse ieh gern IIerrn Ménétriés, seine Absicht durchzuführen, die Ausbeute Wosnesensliys zu veröfentlichen, indem dieselbe fast ausschliesslich sich auf Californien beschränkt, und es dürfte Herrn Ménétriés nicht zuwider sein, wenn ich jezt aus den, auf den Inseln Sitkha und Unalaschka, von ihm ganz fremden, mit mir aber befreundeten Personen cingesammelten Käfern, einen Nachtrag zu meiner früheren Bearbeitung der coleopterologischen Fauna jener Striche des nordwestlichen Amerikas lieferte.

Der Magister der Philosophie Uno Cjoncens, der sich, als evangelischer Pastor, fünf Jahre auf Sitkha aufgehalten, ist neulich uach seinem Vaterlande Finuland heimgelsehrt und hat alle die vou ihm auf Sitkha und Unalaschka gesammelten Insecten, sowohl als anch die Käfer, die er aus Californien im Tausch erworben, und die er selbst \%wischen Ochotsk und Jakutsk und an den Ufern des Lena-Flusses in Sibirien zusammengebracht, mir mit der grössten Freigebigkeit über-

$\left({ }^{*}\right)$ Archiv fïr Naturgeschichte, gegründet von Wiegmann. 10 Jahrg. p. 256. 
lassen, wofür ich mir es zur angenehmsten Pflicht mache, Herrn Cygnrous meine ganz besondere Er"kemntichlieit hiemit öfentlich abzustatten. Er hat meine Sammlung nicht nur mit recht vielen, bis jezt darin vermissten und durch Eschscholtz schon bekannt gewordenen Arten von sitkha und Unalaschka vervollständigt, sondern auch mit mehreren auf diesen Inseln vorher gar nicht beobachteten Species bereichert, welche ich auf der nehmlichen Weise, wie ich bereits meine frühere Abhandlung gleichen Gegenstandes verabfolgte, vorläufig nur durch Diagnosen näher zu bestimmen mich bemühen werde, in der Hoffnung, dass ich sie dadurch gewissermassen meinen entomologischen Collegen erkenntlich nachen dürfte, so manche Schwierigkeiten sich auch, bei dem gegenwärtigen Stande der Entomologie, dieser Methode der descriptiven Bearbeitung entgegenstellen möchten.

Wir wissen schon aus den von Reisenden auf der Insel Sithha und den Aleutischen Inseln $\left({ }^{*}\right)$ früher gemachten entomologischen Ausbeuten,

(") In den Schriften des hochverdienten Professor Germar finden wir übcrall, wo er der Insel Sitkha erwähnt, unrichtigerweise: habitat in insula Alcutorum Sitcha. Die Aleutischen Inseln und Sitkha sind doch nie zu einer Inselgruppe gerechnet worden und können es auch füglich nicht werden, indem sie von einander weit entfernt und von verschiedenen Völkerstämmen bewôhnt sind, jene von den Aleuten, diese aber von 
dass diese Inseln einige nur wenige Käfer-Arten mit einander gemeinschaftlich beherbergen, und bis jezt waren nur zwei Species genannter Inseln anf dem Russisch-Asiatischen Continente gefunden worden, namentlich Amara remote-striata Eschsch.und Hydroporus griseo-striatus De Geer; von den durch den Handels-Verkehr über die ganze Erde verbreiteten Insecten kann hier natürlicher Weise keine Rede sein. Herr Cygnous ist jezt der erste, der den auf Kamschatka von Eschscholtz entdeckten Cryptohypnus littoralis in beträchtlicher Menge auch auf Unalaschka einfing, weshalb ich es für nöthig erachte, denselben in diesem Nachtrage einen Platz cinzuräunen. Durch die Ausbeute des Herrn Cygnceus auf Unalaschka lernte ich auch den wahren Ludius lobatus Eschsch. wäher kennen, dessen er dort in mehrereu Abänderungen habhaft wurde. Ich hatte nehmlich, von der Augabe in Dejoans Catalog irre geleitet, in meinem Beitrage zur Käfer-Fauna der fraglichen Gegenden, als Diacunthus lobatus Eschsch., den caricinus desselben Verfassers aus Sitha aufgenommen, welchen Herr Professor Germar nachher als Corymbites caricinus ganz treffend beschrieben hat. Bei genauerer Untersuchung ergab es sich aber, dass diese beiden Ela-

den Kaliuschen, zwei cinander ganz fremde Menschenracen, die nie verwechseti werden dürlen. 
teren zwei verschiedene ologleich sehr nahe verwandte Arten sind, und es erheischte dieser wichtige Umstand eine Umarbeitung der Diagnosen genannter beider Species, dic ich deshalb ebenfalls in diesem Nachtrage einschalten musste.

Als Berichtigungen zu meinem früher gelieferten Beitrage zur Käfer-Fauna der Aleutischen Inseln, der Insel Sitkha und Neu Californiens, muss ich schliesslich erwähnen, dass Seite $196(24)$ No 3 S Anchomenus ovipennis Eschsch. in A. rotundipennis Motschulsliy und No 39) Anchomenus rugiceps in A. ovipennis Eschsch., nach Herru von Motschulsly's wohlgegründelen Bemerkuug (Bullet. 184j p. 339.), zu verïndern wïren. Das von mir als $A$. rugiceps beschricbene Exemplar des $A$. oripennis hat wahrscheinlich zufilliger Weise einen gerumzelten Kopf gehabt, denn unter den von Merrn Cygnaus mir ertheilten Insecten aus Tord-Californien waren \%wei Stücke der nehmlichen Art mit ganz glatiem Kopfe, die mit der von Motschulsky angefurtigten Beschreibung des A. ovipennis vollkommen übereinstimmen, weswegen aus der von mir aufgestellen Art-Diagnose die Worte capite incequali rugoso gegen capile levigato vertauscht werden müssen. Dagegen hat Herr von Motschulsky völlig unrecht in seiner Angabe, dass mein Lachnophorus elegantulus kein Lachnophorus sei. Wenn man etwas mit so apodictischer Bestimmtheil, wie INer von Motschulsliy sich hierüber geëussert, herausstellt, müsste man 
doch dic Sache näher untersuchen, und da ich jezt diesen Kafer durch Herrn Cygnceus erhalten habe und ihn einer genauen Prüfung unterwerfen konnte, musste ich wohl glauben, dass Herr von Motschulsky vielleicht nie einen Lachnophorus gesehen hätte. Ich hatte wohl schon bei der Beschreibung dieses Lachnophorus, der mit Tachypus mediosignatus Ménétr. freilich identisch ist, angemerk, dass die Palpen etwas verschieden von den für diese Gattung angegebenen Charakteren wären. Es verhält sich auch so, dass das Endglied der Maxillen-Taster, das nach Dejean länger als das vorlezte sein sollte, in der That kürzer ist; allein Dejean hat wahrscheinlich die Charakteristik der Gatiung nach dem Lachnophorus bipunctatus Gory (sexpunctatus Dej.) errichtet, bei welchem dieses Endglied der Taster sich länger als das vorlezte erogibt. Der $\mathbb{L}$. pilosis Eschsch. wähert sich hierin schou mehr dem elengantulus, indem diese Tasterglieder bei jenem von gleicher Länge sind, und ich besitze noch einen Lachnophorus aus Columbien, der in Hinsicht der Maxillen-'Taster mit dem clegantulus vollkommen übereinstimmt. Die parallelen Flügeldecken, die über den ganzen Köper gleichmässig vertheilten Haare (wohcr auch der Galtungsname ganz zweckmässigg gegeben wurde) und die Flügeldecksstreifen, die nach den Seiten und hinten überall vollständig und zur vorderen Hälfte grob, dagegen zur hinteren fein punktirt sind, stehen im- 
mer als natürliche Charaktere da, welche der elegantulus mit den übrigen Lachnophorus-Arten gemeinschaftlich hat und die schwerlich erlauben würden, diese Species in die Bembidien-Untergattung Tachypus auf irgend eine Weise hineinzuschalten.

1. Iomalota granulata: Linearis, depressa, aterrima, nitida, parce pubescens, antennis longioribus, extrorsum incrassatis pilosis, thorace subquadrato, coleopteris parum angustiore, posterius longitudinaliter impresso, elytris grauulatis, abdomine levigato, tibiis tarsisque fusco-testaceis.

$$
\text { Longit. } 1 \%{ }_{5} \text { lin. Latit. } 1 \% \text { lin. }
$$

llabitat in insula Unalaschka.

Statura fere H. Maritimae Eschsch., sed major, nigrior, parcius et longius pubescens, antennis longioribus inulto validioribus elytrisque grauulatis diversa.

2. Boletobies biserintus: Piceo-testaceus, nitidus, antennarum basi, thorace, elytris, abdominis segmentorum marginibus pedibusque ferrugineo-testaceis, elytris disperse punctatis, vitta longitudinali media infuscata, seriebus dorsalibus duabsas, una suturali, ahtera media multipunctatis.

$$
\text { Longit. } 1 \% \text { lin. Latit. } \% \text { lin. }
$$

Habitat in insula Sitkha. 
Statura B. rygme Fabr. affinis, sed color alius et elytrorum panctura distinctus.

3. Staphylinus crassus: Linearis, apterus, niger, nitidus, capite thoraceque glabris Jevissimis, collari postice profunde punctato, thoracis angulo antico utrinque puncto profunde impresso, elytris thorace brevioribus, strigulosis, parce pilosis, apice obliquis, angulo externo rotundato, abdomine punctato-ruguloso, longius griseo-piloso, antennis, tibiis tarsisque fusco-piceis.

Lungit. 61/2 lin. Latit. $1 \frac{1}{2}$ lin.

Habitat in insula Unalaschka.

Statura crassa, elytris brevibus obliquis et defectu ala. rum a reliquis hujus generis speciebus alienus, ad Fam. VIII Gen. et Spec. Staphyl. cel. Erichsonii adnumerandus.

4. Quedrus longrpennis: Niger, nitidus, segmentorum abdominalium marginibus ano tarsisque rufo-ferrugineis, elytris thorace fere duplo longioribus glabris sublievibus, rufis, medio infuscatis, thoracis seriebus dorsalibus tripunctatis, punctis subtilibus.

\section{Longit. $2 \%$ lin. Latit. $\%$ lin.}

IIabitat in insula Unalaschka.

Q. plagiato colore subsimilis, magnitudine minore et elyo tris multo longioribus diversus. 
5. Cryptohypeus attromaisis Eschecholla: Oblongus, gibbosus, supra fusco-reneus, subtus fuscus, thoracis elytroruncue marginibus, antennis pedibusque pallide testaceis, thorace lateribus rotundatis antrorsum leviter sinuatis, sparsim punctato, elytris profunde striattis, interstitiis granulatis.

Longit. 4, 51; lin. Latit. $12 / 5,2$ lin.

Dejean, Cat. 3-me édit. P. 105. Gerimar, Zsitschrilt V. p. 137. 3.

IIrolithus hittoralis Eschscholtz in Thon Entom. Arihiv. II. I. p. 33.

In peninsula Kamschatka a D. Eschschollz detectus, sed occurrit etiam in insula Unalaschka non infrerquens.

6i. Dincantuus Ciricinus Eschescholtz: Elongalus, plumbeo-niger, dense griseo-pubescens : pedibus piceis, thorace elongato, sublineari plauiusculo, angulis posticis productis, modice divaricatis apice truncatis, elytris punctatostriatis, antemis serratis, articulis tertio ct quarto æqualibus.

Longit. $4 \frac{1}{2}$ lin. Latit. $1 \frac{1}{2}, 1 \frac{2}{5}$ lin.

D. Lodstus Mannerhcim Beiträge zur Käferfauna p. 71. 149. (Bullet. de la Soc. de Moscou 1843. p. 243. 149.). Luduos caricious Eschscholtz, Dejean, Cat. 3-me édit p. $10 \%$ Cormarites caricinus. Germar, Zcitschrift. IV. p. 63. 20. Habitat in insula Sitkha. D D. Eschschollz et Kuprianolf: 
7. Diacantuus robatus Eschischollz: Oblongus, niger, sub-xneus, griseo-pubescens, thorace pone medium lateribus nonnilil dilatato, dorso subconvexo, augulis posticis divaricatis planiusculis, minus productis, carinulatis, apice truncatis, lobo medio marginis postici emarginato, elytris punctato-striatis, antennis serratis, articulo tertio quarto longiore.

Longit. $3 \%, 5 \%$ lin. Latit. $1,1 \%$ lin.

Elater lobatus Eschscholt Entomographien p. 69. 41.

Lumus lobatus Eschscholtz in Thon Entom. Archiv. 1I. I p. 34

Var. b. ut a, sed humeris rufis.

VAr. c. elytris fusco-castaneis, basi ferrugineis, cetera ut in a.

$V_{A R}$. d. elytris tibiisque ferrugineis, cetera ut in a.

IIabitat in insula Unalaschka sat frequens.

$\Lambda$ precedente differt statura minus elongala, punctura minus crebra at profundiore, pilositate rariore, thorace breviore, convexiore, lateribus pone medium nomnihil dilatato, angulis posticis multo brevioribus, latioribus, lobatis, magis divaricatis et carinula elevata utrinque munitis, basi medio emarginato et antennarum articulo tertio quarto longiore.

8. Rinagonycina sericata: Lineari-clongala, nigra, dense grisen-puberula, palpis, antennarum ar- 
ticulis tribus baseos greniculisque obscure testaceis, thorace quadrato, dorso canaliculato, utrinque longitudinaliter elevato.

\section{Longit. 3 lin. Latit. $1 \frac{1}{4}$ lin.}

Habitat in insula Sitkha.

Ru. PINIPULE Eschsch aflinis, sed latior, thorace multo lireviore, latiore, quadrato et elytris obsoletius rugoso-punctatis, multo brevius et donsius griseo-sericatis diversa.

9. Rhagonycha binodula: Lineari-elongala, lola nigra, subtiliter griseo-puberula, thorace brevi, transverso, explanato-marginato, medio profunde canaliculato, disco postico utrinque elevato levissimo nitido, elytris subtiliter rugoso-punctatis, lineis binis elevalis.

Longit. $2 \frac{1}{2}$ lin Latit. 1 lin.

Mas: capite, oculisque majoribus, antemnis rassis, Jongioribus, thorace lateribus subrectis.

Femina: capite angustiore, antennis brevioribus, tenuioribus, thorace dateribus silurotundato.

IIabitat in insulis Sitkha et Unalaschka.

Statura fere Rur. atrae Linné et ejus magnitudine, sed latior, tuta nigerrima et thoracis structura distincta.

Corynetes ruficolels Fabricills.

Fabricius Systema Eleuther. 1. p. 286. 3.-Herbst, Natur. 
syst. aller Ins. IV, p. 152. 3, Tab. 41. fig. 9.- Schünherr. Syn. Ins. II. p. 51. 4.-Dejean, Cat. 3-me édit p. $12 \%$.

Necroria ruficolus Olivier Entomologie IV. 76. bis p. G. 3. Tab. 1. fig. 3. a. b.--Latreille Hist. nat. des Crust. et des Ins. IX. p. 156. Gen. Crust. et Ins, I. p. 274. 2.-Stephens Illustr. of British Entomology III. p. $32 \%$. Manual of British Coleopt.p. 198. 1569. Curtis British Entomology VIII. pl. 350.

VAr. elytris colore magis nigrescente, basi multo brevius rufa, striis subtiliter interstitiis vero profundius punctatis, pilis longioribus, in series condensatis, ita ut elytra griseo-lineata appareant. Vix vero species diversa?

Longit. $2 \frac{1}{2}$ lin. Latit. $1 \frac{1}{4}$ lin.

Habitat in insula Sitkha.

10. Negrophonus defodiens: Niger, supra glaberrimus, antennarum clava concolore, thorace subtiliter punctulato, lateribus sinuatis, elytris profundius remote punctulatis punctis aliquot majoribus sparsis, fascia media dentata maculaque postica antrorsum tridentata aurantiacis, pectore flavido-piloso, abdomine glaberrimo, tibiis posticis rectis.

Longit. $6,8^{2} / 5$ lin. Latit. $3,3^{5} / 4$ lin.

Mas: capite majore, pone oculos dilatato, fronte profunde tricanaliculata, thorace antrorsum nonnihil dilatato.

Femina: capite minore, pone oculos haud di- 
latato, profundius punctato, fronte canalicula media deficiente, thorace antrorsum vix lattiore.

VAr. b. elytris maculis sex parvis aurantiacis, fascia nempe antica in maculas duas irregulares interrupta et macula tertia ante apicem transversim oblonga.

Habitat in insula Sitkla.

N. sortuorus Fabr. similis, sed antennarum clava minore, elytris remotius punctatis et fasciis angustioribus magis dentatis distinctus.

11. Spherites politus: Ovatus, convexus, obscure æeneus, nitidissimus, elytris apice oblique truncatis, punctato-striatis, interstitiis lavissimis, corpore sublus pedibusque nigro-piceis.

Longit. $2 \frac{1}{2}$ lin. Latit. $1 \frac{1}{2}$ lin.

llabitat in insula Sitkha

Sphó glabratn Fabr. valde similis, sed paulo longior, angustior, minus rotundatus, elytris profunclins punctatostriatis interstitisque omnino lævibus diversus; color paginæ superioris, presertim thoracis, etiam magis cupreus.

12. Nitidula truncatella: Linearis, depressa, ferruginea, punctatissima, oculis, nigris, thorace elytrisque disco infuscatis, pectore abdomineque piceis, elytris apice truncatis.

Longit. 11/2 lin. Latit. 1/2 lin.

IIabitat in insula Sitkha. 
N. oblonga Iferbst proxima staturacue parallela ei affinis, sed longior et fortius punctata.

13. Atomaria fulvipennis: Ovata, globosa, nigra, glabra, thorace orbiculato marginato, crebrius punctato, elytris valle convexis, fulvo-testaceis, remote et profunde punctatis.

Longit. $\%$ lin. Latit. $1 / 3$ lin.

Habitat in insula Unalaschka.

14. Dendrophagus Cygnzr: Lineari-elongatus, depressus, niger, nitidus, thorace oblongo crebre punctato, lateribus integro, dorso bisulcato, elytris profunde crenato-striatis.

Longit. $3^{1 / 2}$ lin. Latit. ${ }^{3 /}$ lin.

Habitat in insula Sitkha.

D. crenato Schönh. augustior, colore nigro, antennis tenuioribus, thorace longiore, lateribus haud sinuato et elytris profundius crenato-striatis diversus.

Donacia Germari Eschescholtz.

Mannerheim Beitrag zur Kaferfauna p 134. 281. (Bullet. de la Soc. de Moscou 1843. p. 306. 281.).

Var. c. cupreo-renea, femoribus posticis cingulo fusco notatis, cetera ut in a.

15. Callidum dimiatum: Brumeo-piceum, subtiliter punctulatum, pilis nigris pubescens, antennis pedibusque obscure testaceis, thorace brevi subcylindrico, elytris basi thorace pau- 
to latioribus, dimidiatim antice fusco-testaceis, apice singulatim rotundatis, femoribus valde clavatis.

\section{Longit. 3\% lin. Latit. 1 lin.}

Habitat in insula Unalaschka.

Thorace subcylindrico ad Clytos appropinquat, sed ob antennarum et reliquarum partium structuram Callidiis associandium esse mihi videtur. 


\section{GORIG INDA.}

Pag. 12. Zeile 25 statt: Corynetes ruficorus lies:

9. b. Conynetes ruficollis.

Pag. 15. Zeile 18 stalt: Donacha Genmar lies:

14. J. Donacia Germarr. 



\title{
ZWEITER NACHTRAG
}

\author{
ZUR \\ K A E F E R - F A U N A
}

DER NORD-AMERIKANISCHEN LAENDER DES RUSSISCIIEN REICHES,

voN

Graf C. G. Mannerheim.

$\Rightarrow 0$

MOSCAU.

Ix der Buchdrucberei der haserlichen Universitä.

1852. 



\section{ZWEITER NACITTAG}

ZUR

\section{MiLWWR-FUUVI}

\section{DER NORI) - AIIERIK.INISCIEN LAENDER DES RUSSISCHEN REICHES,}

ron

Graf C. G. minnerheim.

Sechs Jahre sind bereits verflossen, seitdem ich meinen Nachtrag zur Käfer-Fauna der Aleutischen Inseln und der Insel Sitkha unserer hochverehrten Kaiserlichen naturforschenden Gesellschaft einsandte, die in ihrem Bulletin für das Jahr 18.46 diese Abhandlung eines Platzes würdigte. Seit dieser Zeit wurden noch beträchtlichere Entdeckungen zur Ergänzung genannter Fauna gemacht, die mich bewogen haben, einen zweiten Nachtrag zu derselben auszuarbeiten und dem entomologischen Publicum vorzulegen. Der gewesene Gouverneur der Besitzungen der Russisch - Amerikanischen Compagnie, Contre-Admiral 
A. Etholén, gegenwärtig einer der Directoren dieser Compagnie zu St. Petersburg, führt noch immer fort, seine rastlose Wirksamkeit nicht blos auf mercantile Geschäfte beschränkend, alles aufzubieten, um die Kiunde dieser entlegenen Gegenden des grossen russischen Reiches auch in naturwissenschaftlicher Hinsicht zu vervollständigen. Die Entomologie hat es besonders dem unermüdeten Eifer dieses ausgezeichneten Mannes zu verdanken, dass er allen im Dienste der Compagnie angestellten Aerzten zur Pflicht aufgelegt, dem Einsammeln der Insekten eine besondere Aufmerksamkeit zu widmen. Auf dem ersten in den letzten Jahren nach Sitkha abgesegelten fimnländischen Schiffe, befand sich in ärzllicher Bezichung der Magister der Chirurgie A. Pippingskäld, auf dem zweiten der Doctor der Medicin A. Frankenhaeuser, ron welchen der erstgenannte schon längst heimgekehrt ist, Doctor Frankenhaeuser aber in den am stillen Oceane gelegenen Ortschaften der russisch-amerikanischen Compagnie sich noch fortwährend aufhält. Herr Pippingsköld hatte die Gefälligkeit aus seiner auf der Insel Sitlha gemachten Käfer-Ausheute mir alles zu überlassen, was mir nur bemerkenswerth schien, mit der Bedingung, die als neu darin vorgefundenen Arten bekannt zu machen. Im Begriffe, diesem von ihm geäusserten Wunsche Folge zu leisten, wurde ich im Sommer des vorigen Jahres durch eine vom Iferrn Doctor Frankenhaeuser aus Sitkha glücklich eingetroffene Sendung Käfer auf das angenehmste überrascht, indem es mir nicht bekannt war, dass derselbe sich mit der Insektenkunde je beschäftigt hatte. Diese Sendung war an Reichthum der Arten und Individuen gewiss die vorzüglichste, die bis jetzt aus diesen Gegenden 
mir mitgetheilt wurde; die ganz besondere Sauberkeit in der Behandlung sogar der kleinsten Stïcke, und die bei jeder Art zugefïgten schriftlichen Bemerkungen über die Umstände, unter welchen sie gefunden war, zeigten in hervorragender Weise, wie sehr Doctor Franlienhacuser spinem Berufe als Käfer-Sammler entsprochen hatte. Ich verdanke obenerwähnter Freigebigkeit der Herrn Pippingskïld und Frankenhaeuser eine beträchtliche Reihenfolge bisher ganz unbekannter ausgezeichneter Arten, ja selbst einige neue Gattungen, für welche ich es mir zur angenehmsten Pflicht rechne, diesen Herren meine besondere Erkenntlichkeit hier öffentlich auszudrücken. - Kaum war ich aber mit der hritischen Durchmusterung der Frankenhaeuserschen Ausbeute fertig, als mein verehrter entomologischer Freund, $F$. W. Mäklin, Candidat der Philosophie, mir anzeigte, dass bei ihm eine ebenfalls auf der Insel Sitkha gemachte, beträchtliche Käfer-Sammlung von dem gewesenen Conducteur des finnländischen Bergwese'ns, J. Holmberg, eingegangen war. Iferr Mäklin schlug mir also vor, die vom IIerrn IIolmberg etwa gemachten neuen Entdeckungen auch in meine beabsichtigte Abhandlung einzuschalten und natürlicher Weise wurde dieses freundschaftliche Anerbieten von mir mit besonderer Freude angenommen. Herr IIolmberg hatte sich schon längst als ein tüchtiger Insekten-Sammler bewährt, was anch die von ihm auf Sitkha zusammengebrachte grossartige Ausbeute im entomologischen Fache und seine, Iferrn Mäklin mitgetheilte Notizen über die Lebensart der von ihm gefundenen Insekten jetzt vollkommen bestätigen.

Mit diesen von drei meiner verehrten Landsleute erhaltenen reichen Materialien ausgestattet, schritt ich zum Werke; 
allein eine seit drei Jahren mehr und mehr zunehmende Schwäche meiner Augen versagte mir den Gebrauch der nöthigen Apparate, um die kleinsten Gegenstände gehörig zu untersuchen. In dieser für einen Entomologen ganz besonders schlimmen Lage wandte ich mich an IJern Mäklin mit der Bitte, mir bei den Minutien, in deren Kemutniss und wissenschaftlichen Auseinandersetzung er bereits eine Fertigkeit erworben, die ihn sicherlich zu einem der ersten Entomologen unserer Zeit erheben wird, behülflich zu sein. Herr Mälilin hat in dieser Bezielıung fast alle Staphylininen und noch andere der kleinsten Käfer-Arten für liese $\mathrm{Ab}$ handlung charakterisirt und die dazu nöthigen Diagnosen angefertigt. Meinen ganz besonderen Dank diesem würdigen Mitarbeiter für den dadurch erhaltenen wichtigen Beitrag abstattend, habe ich auch alles, was ich ihm verdanke mit "Mäklin» im Texte bezeichnet.

Seitdem ich meine über die Käfer der Aleutischen Inseln, der Insel Sitkha und Neu-Californiens erschienene Fauna veröffentlichte, hatte Ierr Obrist Lieutenant von Motschulsky, im Bulletin unserer Moskauer naturforschenden Gesellschaft für 1845 und 1851 einige Käfer-Arten aus Sitkha und Unalaschka bekannt gemacht; auch sind desgleichen von ihm in seinem neulich gedruckten Kataloge der Käfer Russlands, bis jetzt nur die Carabicinen enthaltend, etliche neue Species aus den nordamerikanischen Ländern des russischen Reiches aufgenommen worden. Um meiner gegenwärtigen Abhandlung eine gehörige faunistische Vollständigkeit zu geben, war es mir also nöthig, diese Käfer in Augenschein zu nehmen, damit ich sie mit behuflichen Diagnosen unter die übrigen entomologischen Producte der fraglichen Gegenden 
einreihen konnte. Durch die freundschaftliche Bereitwilligkeit, wovon Herr v. Motschulskiy mir schon so manche Beweise gegeben hatte, belebt, theilte er mir auch fast alle Typen der von ihm beschriebenen Arten mit und setzte mich in den Stand, meinen $Z_{w e c k}$ womöglichst verfolgen zu können. Seine Myosodus femoratus, Emmenastus rugosus, Steropus rugulosus, Cybocephahus? unicolor und Plilium (nachher Acratrichis) Sitkaense blieben mir, unter den im Bulletin bereits erwähnten Species, demohngeachtet doch zur eigenen Ansicht unbekannt. Die beiden ersten von diesen konnte ich, bei fehlenden Diagnosen, in die Reihenfolge der charakterisirten Arten gar nicht aufnehmen; was die übrigen aber anbetrifft, so sind sie von mir mit Herrn $v$. Motschulskys eigenen Diagnosen in dieser Reihenfolge an ihren gehörigen Stellen eingeschaltet. Ebenso habe ich auch mit dem in seinem Kataloge eingetragenen Cychrus reticulatus verfahren; allein für die übrigen darin ohne Diagnosen aufgenommenen Arten komte dieses bis jetzt nicht geschehen. Zwar habe ich viele Ursache zu bezweifeln, dass der Myosodus femoratus, wegen seiner der Hochgebirgs-Fauna eigenthümlichen Form, aus Sitkha stammen könne; Herr v. Motschulsky hat nemlich selbst diesen Käfer mit Fragezeichen sowohl für Sitkha als für Irkutsk in seinen Katalog eingeführt, und ich bin daher fast überzeugt, dass das fragliche Insekt offenbarer zu den sibirischen Alpin-Formen gehört, weswegen ich die Art aus der Sitkhaer Fauna herauszustreichen mich bewogen gefunden habe.-Gleichfalls kann ich nicht zur Gewissheit gelangen, dass Sitkha den Agonoihorax splendidulus Kotsch. (die Käfer Russlandsp.68.) gemeinschaflich mit Kamtschatka beherbergen sollte, und 
dies um so weniger, indem unser verdienstvolle Entomolog als Synonym einen A. impressipennis? Mannerh. aus Sitkha beigefügt, den ich selbst nicht einmal kenne. Uebrigens wird der gedachte A. splendidulus vom Herrn Baron $v$. Chaudoir unter Anchomenus impressus Panz., der in ganz Sibirien vorkömmt, gebracht. (Bullet. de la Soc. de Moscou. 1850. III. p. 123. 39.) Fast ebenso schwer kann ich glauben, dass der Emmenastus rugosus wirklich von Sitkha gekommen sei, weil noch keine zur Sippe der Melasomen unter den Heteromeren gehörende Art dort von so vielen fleissigen Sammlern entdeckt worden ist. Wegen der ausgezeichnet schönen Rosalia funebris Motsch. wage ich dagegen keine so ganz bestimmte Meinung auszusprechen; denn ihre europäische Stammverwandte, die Rosalia alpina, lebt nach Mülsant als Larve nicht nur im Holze der Buchen, sondern auch der Fichten, weswegen die funebris ebenfalls im Innern der grossen Nadelholz - Waldungen von Sitkha sich finden möchte. - Die vom Herrn Baron $v$. Chaudoir im Bulletin unserer Gesellschaft (1850. III. p. 135. 6.) beschriebene Feronia commixta aus Sitkha ist zwar auch nicht meiner Aufmerksamkeit entgangen; da dieser Art aber keine lateinische Diagnose beigefügt wurde, musste ich sie aus dem Texte der Beschreibungen weglassen und ihrer bloss erwähwen. Schliesslich bleibt unter den vom seligen Eschscholtz auf Sitkha gefundenen Käfern nur noch der Aegialites debilis uns gänzlich unbekannt und ein wahres Räthsel, an dem wir uns die Köpfe zerbrechen. Dejean schaltete in seinem Kataloge diese Gattung zwischen Gibbium und Mastigus ein; es ist daher nicht auszumitteln, ob sie den Ptinioren oder den Scydmaenen zugesellt werden soll, weshalb ich auch in 
dieser Abhandlung derselben ferner keiner Erwähnung thun konnte.

Aus triftigen Rücksichten, die ich schon in der Vorrede meines ersten Nachtrages zur Käfer-Fauna der Aleutischen Inseln und der Insel Sitliha (Bullet. 18 16. 11. p. 503.) angegeben habe, beschränke ich mich fortwährend bei der faunistischen Bearbeitung der Käfer aus den vom stillen Ocean bewässerten Ländern Ford - Amerikas, nur auf das dem russischen Reiche gehörende Territorium dieses Welttheiles, in welchem nicht nur die Insel sondern auch ein beträchtliches Gebiet des Continents inbegriffen sind. Daher habe ich auch einige, am Meerbusen von Kenai (Cooks inlet) in der Nähe der grossen Halbinsel Alaschka, und in der Umgegend von Tschishlkhath (59" X. B.) gefundene KäferArten in die gegenwärtige Abhandlung aufgenommen. Nach den diagnostischen Bestimmungen, die vielleicht bisweilen etwas ungewöhnlich weillüuftig erscheinen dürften,allein, um Beschreibungen zu vertreten, diese Weilläuftigkeit in manchen Fällen erforderten, lasse ich als besonderen Anhang ein Verzeichniss aller bisher aus den fraglichen Ländereien Nord-Amerikas bis jetzt bekannten Käfer folgen, und habe sie dabei in das natürliche System geordnet; das künstliche Dejeansche Käfer-System ist aber wie vorher in der descriptiven Bearbeitung zum Grunde gelegt. In diesem Anhange sind auch die Beobachtungen der Herren Pippingsköld und Franlienharuser über die nähern Umstïnde des Fundes bei jeder Art angegelen worden, wo dieses nicht in der diagnostischen Bearbeitung schon erwähnt wurde. Ich denke auf diese Weise nachkommenden Entomologen, die diese Gegenden besuchen, aus meinen drei Abhandlungen 
ein Handbuch zu bereiten, um die vielen dort vorkommenden interessanten liäferformen leichter aufspüren und auf eigene Hand systematisch bestimmen zu können.

Die Hauptgegenden des Insekten - Forschens in obenerwähntem Landstriche sind bis jetzı die Aleutischen Inseln und die Insel Sitkha gewesen. Die Aleutischen Inseln, wie auch die IIalbinsel Alaschka, sind waldlos; ihre primitive Vegetation besteht hauptsächlich aus Kräutern und ganz niedrigen Gesträuchen, wie einige Salix-Arten, Rhododendron chrysanthum, efliche Ericacéen und Vaccinien, die nur einige Zoll hoch werden; der Boden ist vulcanisch und sandig; die Ufer bieten aus dem Meere aufgeworfene Algen in Menge dar, die viele Käfer unter sich beherbergen. Auf der Insel Sitkha sind die Coniferen vom höchsten Wuchs praevalirend und bilden dichte Urwälder, durch welche bis jetzt die Entomologen nicht gedrungen sind. Diese Coniferen sind Pinus inops Ait., Mertensiana Bong., Sitchensis Bong., Abies Canadensis, eine der picea nahestehende Art und Larix microcarpa; auch Thuja excelsa Bong. und Jumiperus nana finden sich dort. Aus den belaubten Bäumen gehören zur Vegetalion dieser Insel Pyrus diversifolia Bong., Pyrus sambucifolia Cham., Almus viridis und rubra Bong., Salix Sitchensis Bong. und Panax horridum Sm. Noch muss ich der Ilimbeeren auf Sitkha, Rubus spectabilis und Nutkensis, erwähnen, weil ihre Blüthezeit die Ankunft der schönen Colibris bedingt und das Erscheinen dieser Vögelchen auch mit der Entwicklung der Insekten, die denselben zur Nahrung dienen, in Verbindung stehen muss. Der Boden dieser Insel ist theilweise sandig, theilweise in den Urwäldern, wo die Bäume eine Grösse von 亿 bis 5 Fuss im Durchschnitt er- 
reichen, aus vermoderten Vegetabilien bestehend. Ihr Klima ist sehr feucht und es sind wenige Tage im Jahre, wo es nicht regnet. Cicindelen scheinen gar nicht auf Sithha vorzukommen, die Cychren ersetzen die grossen Caraben, keine Histeren, Anthicinen und Oedemeriden sind dort gefunden worden, nur eine einzige Art von der zahlreichen Familie der Scarabaeiden ist bis jetzt auf der Insel entdeckt, auch die Melasomen der Heteromeren, an welchen Californien so ausgezeichnet reich ist, scheinen hier ganz zu fehten, wenn der Emmenastus rugosus Motsch. abgehen würde; allein was am meisten auffällt, das ist der totale Mangel nicht nur aller eigentlichen Chrysomelinen, dazu natürlicher Weise die schon längst durch Eschscholt bekannt gemachten 2 Donacien-Arten und Symeta carinata nicht gerechnet, sondern auch der Coccinellinen, die noch lieine einzige Art haben zum Vorschein kommen lassen. Dieser Umstand ist um so seltsamer, weil im höchsten Norden noch die Insel Novaja Semlja an Käfern nur eine Art Chrysomela aufzuweisen hat und gewöhnlich die Scymmus wenigstens nie in unseren Nadelholz - Waldungen fehlen. Man möchte aus letzterwähntem Ergebnisse fast schliessen, dass die Insel Sithha in der ganz besonderen Lage sich befände, ihre Vegetation von den Aphiden ganz unangetastet zu behalten. Die Carabicinen, Elaterinen und die Borkenkäfer, besonders aber die Staphylininen sind dagegen auf der Insel Sitlha an Zahl sowohl der Arten als noch mehr der Individuen stark vertreten, und das Letzte gilt vorzüglich von allen Käfer-Sippen, die im Aase oder in faulenden animalischen und vegetabilischen Gegenständen leben, wie Silphalen und Nitidularien. Ueberhaupt hat die Käfer - Fauna von Sitkha und die der 
nächstliegenden Länder an ihren Insecten vieles mit unserem Norden Gemeinschafliche und es begegnen sich dort mit diesen einige rein Nord-Amerikanische Bildungen, _ ein Zusammentreffen, das diese Fauna dem europäischen Naturforscher, bei Beobachtung der geographischen Verbreitung der mannigfaltigen Käfer-Formen, gerade zu einer der interessantesten machen dürfte.

•1. Cycinus reticulatus Motschulsky: "niger , convexus; thorace cordato, dorso subgibboso, postice coarctato, transversim fortiter impresso, angulis posticis rectis; elytris subglobosis, reticulato-striatis, antennis validis. " (Motschulsky.)

Longit. 5 lin. Latit. $2 \frac{1}{2}$ lin.

Motschulsky, die Käfer Russlands. p. 90.

$\mathrm{Ct}$ in insula Cnalaschka vel in California habitantem signo interrogationis indicavit 1). Motschulskiy de specimine, quod a D. Eschscholtz obtinuit. Hoc potius in Unalaschka captum fuisse putaverim.

Sec. Cel. Motschulshiy differt a. C. Manginato Eschsch. statıra breviore, latiore, rotundiore et convexiore, antennis validioribus, pedibus longioribus thoraceque postice valde angustato, ante angulos posticos conspicue coarctato, basi supra fortiter transversim impresso. An revera species diversa vel tantum varietas $\mathrm{C}$ manginatr?

2. Carabus Vietiyguovil Adams: oblongo-ovatus, supra nigro - cyaneus, thoracis ely trorumque margine

Die Species, welche ich zur eigenen Intersuchurg nicht gehaht, sint flursh ein bezeichnot. 
laterali rubro-aureo splendido introrsum virescente; thorace subquadrato, remote rugoso-punctato, medio tenue canaliculato, lateribus praesertim posterius reflexo-marginato, angulis basalibus parum productis rotundatis; elytris lineis elevatis longitudinalibus interruptis punctisque interjectis confluentibus valde scabrosis.

Longit. $10 \frac{1}{2}$ lin. Latit. 4 lin.

Adams, Mém. de la Soc. de Moscou. III. p. 170. Tab. XII. fig. 3. - Fischer, Entomogr, de la Russie. 1. p. 98. 19. Tab. IX. fig. 19. III. p. 166. 20. - Dejean, Spec. gén. des Col. II. p. 61. 21. Iconogr. des Col. d'Europe. I. p. 316. 28. Tab. XLI. fig. 2. - Richardson, Faun. Bor. Amer. IV. p. 17. 13. Tab. I. fig. 3. - Motschulsliy, die Käfer liussl. p. 80.

Habitat in continenti Imerices borealis; specimina in littoribus ad Fretum Behringii capta mihi dederunt D. I). $\boldsymbol{K} u$ prianoff et Etholén.

In his individuis Boreali - Americanis thorax angustior, lateribus magis rectis, levius reflexo-marginatis angulisque posticis angustius rotundato-lobatis subdivergentibus et elytra angustiora oblongius elliptica, lineis elevatis minus regularibus, saepius interrupt is superficieque magis rugoso-intricata; vix vero notae sufficiuntur ut ex illis speciem diversam constituere andeamus.

3. Leistus perruginosus, Mammerheim: Bullet. de la Soc. de Moscou 1843. p. 187. 14.

Motschulsky, die Käfer Russl. p. 71.

$V a r$. b. obscure castaneo-piceus, ore, antennis, thoracis margine pedibusque dilutioribus.

lisdem locis ut Var. a, sed rarius occurrit; I). Holmbery.

1. Nebria carboraria Eschscholt: nigra, nitida, pe- 
dibus rufis, tibiis tarsisque infuscatis; thorace brevi transverso, cordato, posterius valde angustato, angulis rectis, margine late elevato-explanato vix rugoso; elytrorum striis profunde exaratis, punctatis, interstitio tertio foveolis quatuor, quinto tribus profunde impressis.

Variat interdum una alterave e foveolis interstitii quinti deficiente.

Longit. $4 \frac{1}{2}$ lin. Latit. 2 lin.

Eschscholtz, Zool. Mlas. V. p. 24. 8. - Motschulsky, die Käfer Russlands. p. 72.

Habitat in insula St. Pauli; D. Wosnesensky. Individua e liamtschatka etiam obtinui, quare ad N. Carbonarim Eschsch, pertinere crediderim.

5. Axchonenus moleis Eschscholt: Mannerh. Bullet. de la Soc. de Moscou. 1843. p. 198. 43.

Agovothonax molus Motschulsliy, die Käfer Russl. p. 67.

Var. b. rufo-brunnea, capite thoracisque disco obscurioribus.

Chaudoir, Bullet. de la Soc. de Moscou 1850. III. p. 103. 6. Archomexus brunxescens Sahlberg in litteris.

Far. c. minor, thorace posterius perparum magis angustato; cetera ut in Var. $b$.

Axchomexus brevuscolus Sahlberg in litteris.-Motschulsky, die Käfer Russl. p. 70.

Ilabitat in insula Atkha, ubi etiam occurrit A. molus Var. a; D. Ferd. Sahlberg.

6. Axcionenus strigicolus: oblongus, depressus, aterrimus, nitidus; thorace breviusculo, elytris duplo angustiore, antrorsum nonnihil rotundato-dilatato, undique subtiliter transversim striguloso, margine po- 


\section{5}

stice tantum reflexo, medio profunde canaliculato, lineaque obsolete impressa obliqua postice utrinque notato; elytris oblongo-quadratis, apice leviter sinuatotruncatis, obsoletissime striatis, interstitiis laevigatis, tertio punctis tribus parum conspicuis.

Longit. $3 \frac{1}{2}$ lin. Latit. $1 \frac{1}{2}$ lin.

IIabitat ad fontes Kaknu in continenti Americes borealis:

D. Frankenhacuser.

A. Bogemanxi Gyllenh. et uctuoso Dej. affinis, sed colore magis atro, thorace angustiore, evidentius striguloso, lateribus antrorsum valde deplanato elytrisque latioribus brevioribusque ab illis sat distinctus.

7. Pterosticius Orinouvu Leach: niger, nitidus; thorace longitudine latiore, postice parum angustato, tenuissime marginato, medio canaliculato, utrinque unistriato, baseos medio impunctato, angulis posticis obtusis; elytris oblongo-ovatis, striatis, striis obsoletissime punctulatis, foveolis quinque vel sex impressis.

Longit. $4 \frac{3}{4}-5$ lin. Latit. $1 \frac{3}{4}-2$ lin.

Omiseus onnomem Curtis, Brit. Ent. I. 15. Stephens Illustr. Mandib. I. p. 114. 3. Tab. VII. fig. 3. Man. of Brit. Col. p. 32. 220. Richardson Fauna Boreali-Americana IV. p. 32.40 .

Habitat in continenti Americes borealis ad fontes Kaknu:

D. Frankenhaeuser.

Pr. vitneo Eschsch. (вonealr Zetterst.' valde aflinis, thorace latiore, breviore, vix cordato, basi medio omnino laevi imprimis distinctus.

"8. Pterosticius Rugulosus Eschscholiz: "apterus, nigro-subaeneus, opacus; thorace subtransverso, lateribus arcuatis, marginatis, angulis posticis obtusis; 
elytris ovatis, subconvexis, profunde striatis, interstitiis inaequaliter foveolatis, subrugosis; antennis pedibusque rufo-ferrugineis." (Molschulsliy).

Longit. 4 lin. Latit. $1 \frac{2}{5}$ lin.

Steropus nugulosus Motschulsky, Bullet. de la Soc. de Moscou. 1845. IV. p. 342. 11.

Stenoderus nugulosus Motschulsky, die Kïfer Russl. p. 55. IIabitat in insula Unalaschka; D. Eschscholtz, sec. D. Motschulsky.

9. Pтевosticuus simil Ménétriés: oblongus, apterus, cupreo-aeneus, nitidus; thorace subquadrato, lateribus antrorsum nonnilil dilatato-rotundato, medio canaliculato, basi utrinque bistriato, angulis subrectis; elytris oblongo-ovatis, striatis, striis subtilissime remote punctatis, interstitio tertio utrinque punctis quatuor minutis impressis; antennarum basi pedibusque obscure rufis.

Longit. $2 \frac{2}{3}-3$ lin. Latit. $1-1 \frac{1}{6}$ lin.

Cryonus smmurs Motscluulsky, die Käfer Russl. p. $ّ 5$.

Habilat in insula St. Georgii; D. Wosnesensky. Specimina in IIuseo meo asservata amicitiae D. Ménétriés debeo, qui etiam locum habitationis hujus insecti mihi indicavit.

P't. empetricolae Eschsch. affinis, sed minor, ut el colore cupreo-metallico, thorace posterius multo minus angustato, elytris longioribus horumque punctis striarum magis remotis ab illo satis diversus.

10. Miscodera ins ignis: elongata, nigro-picea, nitida, antennis, pedibus, thoracis elytrorumque marginibus rufo-piceis; capite thorace vix angustiore, vertice profunde transversim impresso; thorace ovato, pulvinato, 
basi angustato-constricto ibique punctato, dorso canaliculato; elytris leviter punctato-striatis, striis extrorsum obsoletioribus.

Longit. $4 \frac{1}{2}$ lin. Latit. $1 \frac{2}{3}$ lin.

IIabitat in insula Sitliha sub lapidibus rarissime; specimen tantum unicum legit D. Pippingsköld.

Species praestantissima, Ni. secten Poryk. dimidio major, longior; colore, capitis thoracisque forma el elytris totis punctato-striatis diversa.

11. Amara interstitialis Eschscholt:: oblongo-ovata, supra obscure aenea; thorace antice angustato ibique versus latera utrinque linea currata impresso, medio canaliculato, postice utrinque bifoveolato, foreis obsoletissime punctulatis; elytris subtiliter punctatostriatis, interstitiis alternatim plus minusve subelevatis; antennis basi piceis, pedibus nigris.

Longit. $3 \frac{1}{2}-4$ lin. Latit. $1 \frac{2}{3}-2$ lin.

Dejean, Spec. gén. III. p. 472. 13. Cat. 3-ême édit. p. 44.Motschulsky, die Käfer Russl. p. 60.

Ceun intenstitunis Zimmermann in Gisll Faunus I. p. 24.

IIabitat in continenti Americes borealis ad Tschishlkhath; D. Holmberg.

12. Amara melanogastrica Eschscholtz: Mannerh. Bullet. de la Soc. de Moscou. 1843. p. 210.

Linus melaxogastricus Motschulsky, die Käfer Russl. p. 63.

$V a r, b$, elytris rufis; cetera ut in $a$.

In insula Unalaschka a D. Cygnaeo capta.

13. Amara Escuscuoltzi Chaudoir: oblonga, supra nigro-aenea, subnitida; thorace lateribus valde rotun- 
dato, postice subangustato, utrinque bifoveolato, foveis fere in unam conflatis, basi apice(que punctato; elytris oblongis, parallelis, punctato-striatis; antennarum basi pedibusque rufis.

$V a r . b$. elytris rufis, sutura margineque infuscatis.

Longit. $4 \frac{3}{3}-5$ lin. Latit. $1 \frac{1}{2}-1 \frac{2}{3}$ lin.

Leines Escuscholtzir, Chaudoir Bullet. de la Soc. de Moscon. 1837. VII. p. 36.

Linus Eschscholtzir Motschulsky, die Käfer Russl. p. 63.

Habitat in insula St. Pauli et in continenti Americes borealis; D. Wosnesensky: in Kamtschatka; D. Eschscholtz.

Praecedente minor, angustior, convexior et magis parallela; practerea thorace angustiore basin usque lateribus rotundato foveisque confluentibus, elytris longioribus et antennis basi tantum rufis distincta. - Quamvis hanc speciem ad Aм. hy penborenim Dejean (Spec. gén. V. p. 800.77.) retulerit D. Motschulsky, (Bullet. de la Soc. de Moscou 1851. IV. p. 609.) attamen nomen a L. B. Chaudoir datum potius sit conservandum; antennae nempe semper basi tantum rufae, quum in AM. Hypenbonea a Cel. Dejean ut totae rufae discribuntur.

14. II a Ratus nigrinus Eschscholtz: Mannerh. Bullet. de la Soc. de Moscou. 1843. p. 213.80.

Stexolophus nigrinus Motschulsky, die Käfer Russl. p. 22. Var. b. elytris fusco-castaneis; cetera ut in $a$.

Specimen unicum in insula Sitkha invenit D. Holmberg.

15. Trecius spectabilis: oblongo-ovatus, depressus nigro-piceus, ore, antennarum articulo primo, pedibus anoque rufis; capite ovato ore acuminato, fronte utrinque late excavata; thorace subcordato, lateribus reflexomarginato, medio profunde canaliculato, basi utrinque 
foveolato bistriato, angulis acutis prominulis; elytris oblongo-ovalibus, humeris apiceque rotundatis, lateribus marginatis, undique profunde striatis, interstitio tertio bifoveolato.

Longit. 3 lin. Latit. $1 \frac{1}{3}$ lin.

Iabitat in insula Sillha, in truncis putridis rarius; D. D. Pi Pim pingsköld et Frankenhaeuser.

16. Treciles onloxgeles: apterus, oblongrus, rufotestaceus, nitidus; capite oblongo-ovato, fronte levius bicanaliculata; thorace subcordato, lateribus rotundato. tenuissime marginato basi apice parum angustiore angulis rectis, medio profunde canaliculato, postice utrinque foveolato histriato; elytris oblongis subconvexis, sat profunde punctato-striatis, striis extrorsum tenuioribus, interstitio tertio bifoveolato.

Longit. 2 lin. Latit. $\frac{5}{6}$ lin.

Ilabitat in insula Sitkha sub lapidibus non infrequens;

D. D. Frankienhaeuser et Holmberg.

17. Trecius ovipexis Motschulsky: apterus, ovatus, subdepressus, nitidus, rufescens, thorace elytrisque obscurius piceis submetallicis; capite ovato, fronte tricarinata, oculis nigris; thorace transverso subcordato, reflexo-marginato, medio profunde canaliculato, angulis posticis rectis, basi utrinque oblique unistriato; elytris oblongo-rotundatis, sub-orbiculatis, singulo tenuissime quadristriatis; thorace subtus, antennis, pedibus elytrorumque margine pallide rufo-testaceis.

Longit. $1 \frac{2}{3}$ lin. Latit. $\frac{3}{4}$ lin.

Motschulsky, Bullet. de la Soc. de Moscon 18t5. IV. p. Mis. 20. Die Käfer Russl. p. 7 


\section{8}

Habilat in insula Sitkha sub lapidibus rarius; D. Pippingsküld. Specimen mihi datum ad speciem a se descriptam pertinere asseveravit D. Motschulsky.

18. Trecius Califoricus ( ${ }^{*}$ Motschulsliy: apterus, ovatus, depressus, nigro-piceus; fronte bicanaliculata; thorace transverse-quadrạto, lateribus dilatato-rotundato, marginibus subreflexis, angulis posticis rectis prominulis, medio canaliculato, basi utrinque profunde foveolato; elytris fere oblongo-quadratis, apice subIruncatis, singulo quadristriatis, interstitio tertio bifoveolato; ore, antennis, pedibus elytrormmque margine rufo-testaceis.

V ar. b. pallidior, thorace rufo-ferrugineo, elytrorum limbo pedibusque dilutioribus.

Longit. $1 \frac{3}{4}$ lin. Latit. $\frac{3}{4}$ lin.

Motschulskiy, Bullet. de la Soc. de Moscou. 1845. IV. p. 347.

18. - Die Käfer Russl. p. 7.

IIabitat in insula Sitliha sub cortice arborum emortuarum et sub lapidibus; D. D. Frankenhaeuser et Pippingsköld. A. D. Motschulskiy ut species ab illo descripta indicatus.

19. BEM В I IU M N GRIPES Kïby: supra obscure aeneum; thorace subquadrato, lateribus rotundato, basi utrinque bistriato; elytris oblongis, sat profunde punctatostriatis, basi, fasciis undatis macularibus duabus apiceque pallide testaceis, disco punctis duobus utrinque impressis; pedibus obscure piceo-testaceis.

Longit. $2-2 \frac{1}{3}$ lin. Latit. $\frac{5}{6}-1$ lin.

(") Warum Herr von Motschulsky bei der Beschreibung diese Art Californicus genannt, ist mir schwer zu ermitteln, da er nur Sitkha als ihr Vaterland angiebt. 
Notapues nigripes: Richardson Fauna Boreali-Americana IV. p. 57. 83.

Bembidium (Notapius) indistixctum? Eschschollz: Dejean Spec. gén. V. p. 67. 30.

Notaphus indistinctus? Motschulsky, die Käfer Russl. p. 15.

Notaphus Sitchaevsis Ferd. Sahlberg in litteris.

In insula Sitkha passim sub lapidibus a D. D. Ferd. Sahlberg, Pippingsköld et Frankenhaeuser captum.

Cum descriptione Notaph vigripedis in Richardson Fauna Bor. Amer. convenit, pedum colore excepto, sed hi plus minusve nigrescentes, ut congruitatem in dubium revocare non debui. Etiam ad descriptionem Cel. Dejean de Вемв. indistincto factam bene aptandus, nisi elytra distinctius quam in Bемв. ustulato punctato-striata fuerint. Attamen verisimile mihi videtur hanc speciem quoque huc esse referendam.

20. Bembidion ix Certum Motschulsliy: cupreo-aeneum, nitidissimum, breviusculum, subdepressum; thorace brevi transverso, basi vix angustato, angulis obtusis, utrinque postice profunde foveolato, foveola punctata bistriata, dorso canaliculato; elytris breviter ovatis, disco obsoletissime punctato-striatis, stria tertia foveolis duabus profunde impressis; antennis pedibusque nigris.

Longit. $1 \frac{3}{4}-2$ lin. Latit. $\frac{3}{4}-\frac{5}{6}$ lin.

Notapuus incertus, Motschulshy Bullet. de la Soc. de Mosrou. 1845. IV. p. 350. 23. - Die Käfer Russl. p. 15.

Habitat in insula Sitkha, sub cortice truncorum pini et sub lapidibus rarius; D. D. Cygnæus, Pippingstiöld et Frankenhaeuser. Typum descriptionis a se elaboratae etiam communicavit D. Motschulsky.

21. B EM B DIUM BREVE Molschulsky: nigro-aeneum, niti- 
dum, virescenti-micans, leviter convexum; thorace transverso, subcordato, utrinque intra angulos posticos rectos profunde foveolato, bistriato, dorso convexo canaliculato; elytris oblongis, disco sexstriatis, striis profunde punctatis, tertia foveolis duabus minutis impressis; antennarum basi pedibusque piceis.

Longit. 2 lin. Latit. $\frac{3}{4}$ lin.

Penypuus brevis, Motschulstiy Bullet. de la Soc. de Moscou 1845. I. p. 28. 67. - Die Käfer Russl. p. 12.

IIabitat in insula Sitkha, teste D. Motschulskiy, qui mihi ad describendum specimen in Kamtschatka repertum transmisit.

B. Sanberai Dej. proximum, sed latius, thorace praesertim latiore, posterius minus angustato, profundius foveolato ut et sulcis frontalibus magis exaratis distinguendum.

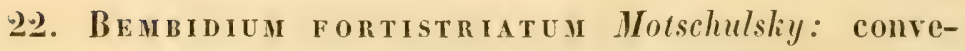
xum, nigrum nitidum; fronte sulcis lateralibus utrinque bistriatis, antice haud connexis; thorace brevi, subcordato, intra basin utrinque bifoveolato, dorso convexo canaliculato, angulis posticis rectis; elytris breviter ovatis, disco profunde punctato-striatis, macula utrinque postica intra marginem testaceo-pallida subrotundata notatis; antennarum articulo primo pedibusque rufo-piceis.

Longit. $1 \frac{1}{2}$ lin. Latit. $\frac{3}{4}$ lin.

Omala fontestriata, Motschulsky, Bullet. de la Soc. de Moscou 1845. IV. p. 352. 26.

Emphanes fortestrutus Motschulsky, dic Käfer Russl. p. 13. IIabitat in insula Sitkha; e Museo D. Motschulsky descriptum.

23. Haliples pantierinus Aube: oblongo-ovalis, fer- 


\section{1}

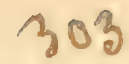

rugineo-testaceus; thorace profunde punctato, antice macula transversa margineque basali nigris; elytris profunde punctato-striatis, interstitiis seriato-punctatis, utrinque septem maculis cum sutura, basi et apice nigro-variegatis.

Longit. $2 \frac{1}{4}$ lin. Latit. $1 \frac{1}{4}$ lin.

Aubé, Spec. gén. des Hydrocanth. p. 29. 17.

Habitat in insula Sitkha; a D. Holmberg in lacu prope oppidum Nov. Archangelsk semel captus.

24. Dytiscus Ooligiuk I Kirby: oblongo-ovatus, subellipticus, olivace-niger nitidus, subtus flavo-testaceus, suturis et segmentorum marginibus nigris, thoracis limbo omni elytrorumque lateribus flavis; thorace lato, medio canaliculato; elytris versus apicem punctis et foveolis sat dense impressis; metasterni lobis acuminatis.

Longit. $14 \frac{1}{2}$ lin. Latit. $8 \frac{1}{4}$ lin.

Richardson, Fauna Boreal. Amer. IV p. 74. 110.

IIabitat in continenti Americes borealis ad Tschishlkhath;

D. Holmberg.

25. Agabus SCAPUlaris: ovalis, subconvexus, niger nitidus, striis anastomozantibus subtiliter reticulatostrigosus; ore antennis, vertice maculis duabus, thoracis margine laterali elytrorumque linea intrahumerali rufo-ferrugineis, tibiis piceo-testaceis, tarsis anterioribus rufo-piceis.

Longit. $3 \frac{1}{3}-3 \frac{\pi}{4}$ lin. Latit. $1 \frac{5}{6}-2$ lin.

Habitat in palude prope oppidum Nov. Archangelsk insulate Sitkhae rarius; D. Frankenhaeuser. 
26. A gabus anturacinus: ovalis, subconvexus, niger subnitidus, striis anastomozantibus subtiliter reticulatostrigosus; ore, antennis, thoracis margine laterali elytrorumque stria intramarginali a medio ad apicem producta ferrugineis, tibiis anterioribus piceo-testaceis, genubus tarsisque obscure rufis.

Var. b. elytris totis nigris.

Longit. $3 \frac{1}{4}$ lin. Latit. $1 \frac{3}{4}$ lin.

Cum praecedente rarissime lectus; D. Frankenhueuser.

Var. b. a D. Holmberg capta.

Proxime praecedenti aflinis, sed statura breviore, sculptura evidentiore ut et colore diversus.

27. HY DROPORUS GONTRA CTULUS: breviter ovatus, subdepressus, ferrugineo-testaceus, subnitidus; thorace dorso obscuriore infuscato, undique subtiliter punctulato, medio tenue canaliculato, serie punctorum majorum intra marginem apicalem notato; elytris crebre punctulatis.

Longit. $1 \frac{1}{2}$ lin. Latit. $\underset{6}{5}$ lin.

IIabitat in insulae Sitkhae aquis rarissime; D. Holmberg.

28. Hy D R POR U S R F I A P ILLUS: oblongiusculus, subdepressus, nigro-piceus subnitidus; thorace antice et postice elytrisque profunde punctatis; subtus niger, capite antennarum basi pedibusque anterioribus rufis, vertice interdum anguste infuscato, elytrorum humeris rufescentibus.

Var. b. praeterea elytris etiam totis rufescentibus, disco plus minusve infuscatis.

Longit. $1 \frac{1}{2}-1 \frac{2}{3}$ lin. Latit. $\frac{2}{3}-\frac{5}{4}$ lin. 
In palude prope Nov. Archangelsk frequens. I). Frankenhaeuser.

29. Hydroporus refinastis: oblongo-ovatus, subdepressus, nigro-piceus subnitidus; thorace antice et postice elytrisque profunde punctatis, subtus niger, fronte antrorsum triangulariter, antennarum basi pedibusque anterioribus rufis.

Longit. $1 \frac{1}{5}$ lin. Latit. $\frac{2}{3}$ lin.

Habitat in insulae Sitkhae aquis stagnantibus rarius; D. Holmberg.

Praecedenti valde affinis et thoracis elytrorumque punctura eique omnino similis, sed statura brevior, capite antice mimus rufo el elytris tolis nigro-piceis distinguendus.

30. Hydroporus eryturostomus: oblongo-ovatus, subdepressus, profunde punctatus, subtus niger, capite nigropiceo, antice rufo; thorace rufo-ferrugineo, disco et postice infuscato, ad basin striola utrinque in elytris continuata; elytris piceo-castaneis, plus minusve confuse, praesertim versus latera, ferrugineo-maculatis; antennarum basi pedibusque rufis.

Var. b. supra rufo-testacea, elytris plus minusve confuse fusco-maculatis, subtus niger, antennis pedibusque rufo-ferrugineis, illis apicem versus infuscatis.

Longit. 1 lin. Latit. $\frac{7}{2}$ lin.

In palude prope Nov. Archangelsk a D. Frankenhueuser sat copiose lectus.

31. Bolitochara nотата Mäklin: rufo-teslacea, subnitida, crebre punctata; antennis apice, capite, macula in medio thorace obsoletissima elytrisque apicem versus indeterminate dilute fuscis; abdomine parcius 
profunde punctato, segmentis tribus primis plaga nigra longitudinali in medio, quarto toto quintoque basi nigris.

Longit. $1_{\frac{1}{2}}^{\frac{1}{2}}$ lin. Latit. parum ultra $\frac{3}{3}$ lin.

IIabitat in insula Silkha rarissime; D. Holmberg. ("Mä̈lin")

32. Tacnyesa fecicola Mülin: atra, opaca, pube tenuissima cinereo-sericea dense vestita; thorace latitudine sua tertia parte breviore, longitudinaliter late impresso; elytris thorace dimidio longioribus et tertia parte latioribus; abdomine lineari, subnitido, crebre subtiliter punctulato; pedibus piceis, geniculis tarsisque dilutius piceo-testaceis.

Longit. $1 \frac{1}{4}-1 \frac{1}{3}$ lin. Latit. $\frac{1}{3}-\frac{1}{2}$ lin.

Sub fucis e mari rejectis in insula Edgecombe non procul ab insula Sitkha initio in. Septembris a D. Holmbery inventa. ("Mälilin")

33. Homalota latevicolis Mäklin: elongata, subdepressa, nigra, nitida, parcius pubescens, subtilissime obsoletissimeque punctulata; thorace omnino fere laevigato; antennis capite cum thorace paulo longioribus, articulis penultimis tribus parum transversis; antennarum basi, elytris, ano pedibusque rufo-testaceis; thorace subquadrato, deplanato, longitudine tertia parte latiore, ante scutellum obsolete foveolato et vix visibiliter longitudinaliter obsoletissime canaliculato; elytris thorace paulo longioribus, versus scutellum angulisque apicalibus nonnihil infuscatis; abdomine anterius parce obsoleteque punctulato, segmentis ultimis laevigatis.

Longit. $1 \frac{1}{3}-1 \frac{1}{2}$ lin. Latit. $\frac{1}{2}$ lin. 
Habitat in insula Sitkha; DD. Pippingsküld et Frankienhaeuser.

Variat antemnarum basi elytrisque totis piceis aut dilutius fusco-testaceis. ("Mäklin")

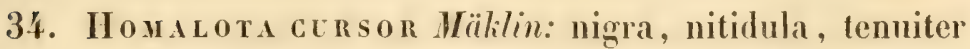
pubescens; palpis, antenuarum basi, elytris, summo apice ani pedibusque testaceis; antennis capite cum thorace longioribus, harum articulis penultimis transversis; thorace longitudine dimidio latiore, crebre subtilius punctulato, longitudinaliter obsoletius impresso; elytris thorace tertia parte longioribus, confertim punctulatis; abdomine basin versus parce punclulato, segmentis ultimis laevigatis.

Longit. $1 \frac{1}{3}$ lin. Latit. $\frac{1}{3}$ lin.

IIabitat in insula Sitkha; D. Holmberg. Varietates elytris nigro-piceis aut omnino nigris ibidem legerunt DD. Pippingsköld et Frankenhaeuser. ("Mäklin ")

35. II о м а ота N Iтекs Mäklin: nigra, nitida, parcius pubescens; antennarum basi anoque piceis, elytris pedibusque testaceis; antennis crassiusculis capite cum thorace longioribus, articulis earum penultimis transversis; fronte late impressa; thorace longitudine dimidio fere latiore, parce punctulato, in medio transversim late at obsoletius impresso (an semper?); elytris thorace paulo longioribus, hoc paulo densius punctulatis; abdomine toto fere omnino laevigato.

Longit. $1 \frac{1}{4}$ lin. Latit. $\frac{1}{3}$ lin.

In insula Sitkha a D. Holmberg sub arborum cortice inventa. ("Mälilin")

36. Homalota Moеsta Mällin: nigra, nilidula, confer- 
lissime punclata, pedibus testaceis; antennis crassiusculis, capite cum thorace longitudine aequalibus, articulis earum penultimis transversis; thorace leviter transverso, latius canaliculato; elytris fuscis, thorace tertia parte longioribus; abdomine basi densius, apicem versus parce punctato, fere laevigato.

Longit. $\frac{4}{5}$ lin. Latit. $\frac{1}{4}$ lin.

Ilabitat in insula Sitkha D. Holmberg. ("Mäklin")

37. Howa ota pratexs is Mäklin: nigra, nitida, densius subtiliusque punctulata, elytris picescentibus, pedibus testaceis; antennis capite cum thorace longitudine aequalibus, articulis penultimis fortius transversis; thorace longitudine plus quam dimidio latiore, obsolete canaliculato; elytris thorace plus quam dimidio longioribus; abdomine basi parce punctato, segmentis duobus penultimis laevigatis.

Longit. $\frac{3}{4}$ lin. Latit. $\frac{1}{5}$ lin.

IIabitat in insula Sitkha, in graminosis; D. Frankenhacuser.

Hom. moesta (juodammodo similis, sed magnitudine minore, antennarum articulis penultimis fortius transversis, thorace breviore, punctura etc. diversa. ("Mäklin")

38. Homalota gryculatälizlin: linearis, depressa, nigra, opaca, dense pubescens; antennarum basi pedibusque piceis, geniculis tarsisque testaceis; capite, thorace elytrisque subtilissime confertissimeque punctulatis; thorace longitudine dimidio fere latiore, canaliculato; elytris thorace plus quam tertia parte longioribus; abdomine sub-nitido, evidentius dense punctato.

Longit. ${ }_{4}^{3}$ lin. Latit. i, lin. 


\section{$27 \quad 309$}

In insula Edgecombe sub fucis e mari rejectis a D. Holmberg inventa. ("Mäklin")

39. Honslota Plaxinis Mällin: subdepressa, nigra, nitida, subtiliter punctulata, tenuiter pubescens; pedibus fuscis, geniculis tarsisque dilutioribus; antennis crassiusculis capite cum thorace longitudine aequalibus, articulis penultimis fortius transversis; fronte interdum excavata; thorace transverso, longitudine duplo fere latiore, longitudinaliter obsoletius impresso; elytris thorace dimidio fere lougioribus; abdomine fere omnino laevigato.

Longit. $\frac{4}{5}$ lin. Latit. $\frac{1}{4}$ lin.

In insula Silkha locis humidis a IDD. Pippingsküld, Franlienhaeuser et IIolmberg inventa. ("Mäklin ")

40. Hолацота в Rеvíscera Mälilin: nigra, nitida, dense punctata, tenuiter pubescens; pedibus antennisque testaceis, harm apice anoque fusco-testaceis; elytris thorace longitudine aequalibus, nonnihil picescentibus; abdomine basi dense, apicem versus parce subtiliusque punctato.

Longit. $\frac{4}{5}$ lin. Latit. parum ultra $\frac{1}{4}$ lin.

Ilabitat in insula Sitkha; DD. Pippingsköld, Frankenhacuser et Holmberg.

Ad divisionem 4. tam operis V. Cl. Erichson referenda; Hom. fungi auct. statura corporis subsimilis, sed multo minor. ("Mäklin")

41. Aleociara Castaneipennis Eschscholtz: Mamerh. Bullet. de la Soc. de Moscou 1843. p. 224. $10 \%$.

Var. $b$. antennarum basi, pedibus, elytris anoque testaceis. In insula Sitkha a D Holmber:g pluries capta. ("Mäklinn) 


\section{8}

42. Aleociara cograta Mälin: nigra, nitida, pube suberecta vestita; antennis pedibusque fuscis; elytris thoracis longitudine, creberrime punctatis; abdomine parallelo, minus dense punctato.

Longit. 2 lin. Latit. vix ultra $\frac{1}{2}$ lin.

In insula Sitkha a D. Holmberg inventa.

A. randginosıe Grav. Erichs. similis, sed pube minus erecta, antennis nomnihil crassioribus, elytris adhuc densius, sed subtilius punctatis et segmentis totis abdominis parcius punctatis, nec singulis basi densius ut in illa. Ab Al. cistaneipenyi Eschsch. Mannerh, in insula Sitkha multo frequentius obvenienti, colore et elytris multo brevioribus facillime dignoscenda. ("Mälilin")

43. Ticinces rigricornis Mannerheim: Bullet. de la Soc. de Moscou 1843. p. 225. 106.

Mas: structura abdominis omnino ut in T. Frigido Erichs. laciniis segmenti inferioris sexti fortasse paulo magis elongatis. ("Mälitin")

Habitat in insula Sitkha, in fungis putridis et in stercoratis minus frequens. D. Frankenhaeuser.

44. TAcuives Frigidus Erichson. Mannerh. Bullet. de la Soc. de Moscou 1843. p. 226. 107.

Femina: abdominis segmento superiore sexto quadridentato, dentibus acutiusculis; segmento inferiore quinto apice integro, non spongioso. ("Mäklin")

lisdem locis cum praecedenti a DD. Frankenhacuser et IIolmberg rarius lectus.

45. Tacinsus circumcinctus Mäklin: niger, nitidus; elytris thorace dimidio longioribus, parcius distinctiusque punctatis pedibusque anterioribus rufo-testaceis; 
elytrorum sutura, marginibus lateralibus et apicem versus latius, abdominis segmento penultimo apice pedibusque posticis piceis.

Nas: abdominis segmento superiore sexto quadridentato, dentibus breviusculis, latioribus; segmento inferiore quinto apice emarginato, margine infra sinum angustissime spongioso.

Femina: abdominis segmento superiore sexto trilaciniato, laciniis latiusculis, intermedia rufo-testacea, apice emarginata.

Longit. 2 lin. Latit. fere $\frac{3}{4}$ lin.

Habitat in insulae Sitkhae stercoratis et fungis haud infrequens; DD. Pippingsköld et Franlienhueuser.

Abdominis structura a congeneribus facillime dignoscitur. ("Mällinn)

46. Tacines maculacoles Mäklin: piceo-niger, nitidus; elytris thorace fere duplo longioribus, brunneoferrugineis; horum basi lateribusque, thoracis lateribus late, antennarum basi, segmentorum abdominalium marginibus anoque late ut et pedibus rufotestaceis.

Mas: abdominis segmento superiore sexto quadridentato, dentibus bevioribus minus acutis; segmento inferiore quarto apice subimpresso, quinto apice emarginato et fortiter impresso, impressione lateribus spongioso, sexto bifido, laciniis elongatis.

Femina: abdominis segmento superiore sexto bilaciniato, laciniis elongatis acutis; segmento inferiore quinto apice truncato integro, sexto tridentato, dente intermedio longiore apice emarginato. 
Longit. $2 \frac{1}{2}-3 \frac{1}{3}$ lin. Latit. $1 \frac{1}{6}-1 \frac{1}{3}$ lin.

In insula Sitkha, in fungis putrescentibus a DD. Frankenhaeuser et Holmberg sat copiose inventus.

Punctura thoracis elytrorumque subtilissima inter congeneres insignis. A T. propinguo (Mannerh. Bullet. de la Soc. de Moscou 1843. p. 226. 108.) magnitudine multo majore, thorace breviore, angustiore, latius ferrugineolimbato et elytris longioribus, omnium subtilissime punctulatis diversus. ("Mälilin")

47. BoLetorius poecilus: elongatus, antice posticeque valde attenuatus, piceus nitidissimus, capite nigro, ore, antennarum basi, thorace, abdominis segmentorum marginibus pedibusque rufo-ferrugineis; thorace elytris angustiore, antrorsum attenuato, basi minute bipunctato; elytris thorace sesqui longioribus laevigalis, nigro-piceis, macula magna subquadrata basali ad medium usque protensa margineque apicali flavotestaceis, stria suturali impressa et serie media e punctis distantibus interdum fere evanescentibus.

Var. b. abdomine rufo-testaceo, ano infuscato; cetera ut in $a$.

Longit. $1 \frac{1}{2}-2 \frac{1}{3}$ lin. Latit. $\frac{7}{12}-\frac{2}{3} \operatorname{lin}$.

IIabitat in insula Sitkha, in fungis putridis frequens;

D. Frankenhaeuser.

Thorace angustiore, elytris laevigatis eorumque serie discoidali punctis magis distantibus obsoletis ut et colore a B. miseriato (Mannerh. Bullet. de la Soc. de Moscou 1846. II. p. 58. 2.) omnino diversus.

48. Otнius Macrocepiatus Eschscholtz: Mannerh. Bullet. de la Soc. de Moscou 1843. p. 227. 110.

$V a r . b$. nigra nitida, antennis, pedibus elytrorumque humeris rufo-piceis. 


\section{1}

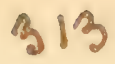

In insula Sitkha sub cortice truncorum pini invenit I). l’ippingsköld.

49. Stapiyzinus crassis: Mammerh. Bullet. de la Soc. de Moscou 1846. II. p. 59. 3.

Íar.b. minor, nigra, elytris abdomineque piceocastaneis, hoc lateribus segmentorumque marginibus, palpis, antennis pedibusque rufis, thoracis punctis antic is minutissimis, parum conspicuis.

Longit. $4 \frac{3}{4}-5 \frac{1}{2}$ lin. Latit. $1 \frac{1}{4}-1 \frac{1}{5} \operatorname{lin}$.

Sub fucis e mare rejectis in insula Sitkha legit D. Frankenhaeuser; raro obvius.

Genus novum nominis Ifaprotes, cui haec species referenda, proposuit D. Ménétriés; characteres rero tales non detegere mihi contigit, ut jure a genere Staphyuno illam separare potuerim.

50. Pinlontuus picipeñis Mälilin: elongatus, niger, nitidus; elytris thorace tertia parte longioribus, subtilius crebriusque punctatis segmentorumque abdominalium marginibus piceis; pedibus testaceis; capite ovato; thoracis seriebus dorsalibus sexpunctatis.

Longit. 2 lin. Latit. fere $\frac{1}{2}$ lin.

In insula Sitkha a DD. Pippingsköld, Frankenhaeuser et Holmberg in stercoratis et in volatu captus.

Phil. aterrimo anct. proximus, sed antennis totis nigris, elytris paulo longioribus, crebrius at minus profunde punctatis diversus. ("Mäklin")

51. Philontudes canescens Mälilin: niger, nitidus; elytris thorace dimidio fere longioribus, opacis, pilis brevibus, rigidis, canescentibus parce, abdomine vero iisden densius et quidem maculatim obtectis; capite 


\section{$66 \quad 32$}

subquadrato, in medio laevi, utrinque vage fortiter profundeque punctato; thoracis dorso bisulcato, sulcis seriebus inordinate sat confertim multipunctatis.

Longit. 4 lin. Latit. $\frac{3}{4}-1$ lin.

In insula Edgecombe sub fucis rejectis medio m. Septembris a DD. Holmberg et Frankenhaeuser pluries inventus. Species inter congeneres eximia, pilositate rigida insigni cum Aleochara sulcicolli Mannerh., iisdem locis eodem tempore inventa, magnam certe offerens similitudinem vitae rationis. ("Mälitin")

52. Quedues plagiatus Mannerheim: Bull. de la Soc. de Moscou 1843. p. 231. 118.

Var. b. thorace toto rufo.

Ex insula Sitkha a D. Holmberg transmissa. ("Mäklin")

53. Quedus erthrogaster: niger nitidus, ore, antennis, pedibus, elytris abdomineque rufis; elytris thorace vix latioribus et parum longioribus, apice extrorsum valde rotundatis, illis abdomineque subtilissime creberrime punctulatis; thoracis seriebus dorsalibus tripunctatis, punctis anticis oblique positis; frontis medio impunctato.

Var. b. ut $a$, sed segmentis abdominis prima et secunda basi infuscatis.

Longit. $3-4 \frac{1}{3}$ lin. Latit. $\frac{5}{6}-1 \frac{1}{4}$ lin.

Ilabitat in insula Sitkha, in rebus animalibus et vegetabilibus putrefactis minus frequens; DD. Frankenhaeuser, Pippingsköld et Holmberg.

Qu. micsimexni (Mannerh. Bullet. de la Soc, de Moscou 18:3. p. 232. 120.) plerumque major, elytris multo densius subtiliusque punctatis, frontis medio impunctato, ut et colore diversus. 
54. QUEDIUS MELAYOCEPHALUS: rufus nitidus, capite pectoreque nigris; thorace antrorsum transversim infuscato, elytris latiore, versus latera subexplanato, valde rotundato; elytris thoracis fere longitudine, apice rotundatis, subtiliter remote punctulatis; abdomine crebrius et subtilius punctulato; thoracis seriebus dorsalibus tripunctatis, punctis anticis oblique positis; frontis medio impunctato.

Longit. $3 \frac{1}{3}$ lin. Latit. 1 lin.

Habitat in continenti Americes borealis ad Tschishlathat D. Holmberg.

A praecedente differt colore, thorace latiore elytrisque remote punctulatis.

55. QUEDIUS AENESC N Malklin: nigro-aeneus, nitidus, antennis pedibusque piceis; elytris thorace paulo longioribus, crebrius subtiliusque punctatis, piceo-casianeis, nonnihil viridi aenescentibus, margine laterali et apicali rufo-testaceis; abdomine densius pubescenti, crebre subtiliter punctato, irideo-versicolore, segmentorum marginibus anoque rufo-brunneis; thoracis seriebus dorsalibus tripunctatis; fronte inter orulos quadripunctata.

Longit. $2 \frac{2}{3}$ lin. Latit. fere $\frac{2}{3}$ lin.

Var. b. elytris totis rufo-brunneis, marginibus dilutioribus.

In insula Sitkha a DD. Pippingstiöld et Holmberg sub lapidibus et in stercoratis inventus. ("Mäklin")

56. Quedus Margixals Mälin: niger, nitidus, antennis, thoracis lateribus, segmentorum abdominalium marginibus, pedibus elytrisque piceis; his thorace longioribus, parce profunle punctatis, sutura margini- 
busque rufo-testaceis; abdomine densius pubescente, minns deuse evidentius punctato, parum irideo-versi-

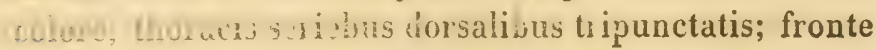
inter oculos quadripunctata.

Longit. $2 \frac{3}{4}$ lin. Latit. $\frac{2}{3}$ lin.

Uabitat in insula Silkiha; in corvo mortuo cepit D. Pippingsköld.

A Qced. amescritr, cui admodum similis est, magnitudine paulo majore, antennis elytrisque longioribus, horum abdominisçue punctura sat diversus. ("Mällin")

57. Stexus maritines Motschulsliy: niger, subnitidus, crebre fortitar punctatus, palpis testaceis, apice fuscis; fronte impressa, utrinque sulcata, interstitio leviter convexo, subeatinato; thorace latitudine paulo longiore, lat ribus in madio dilatato, supra dor so medio canaliculato; elyti is inaequalibus, sacundum suturam torulosis; abdomine parcius subtiliusque punctato; pedibus testaceis, geniculis tarsisque fuscis, horum articulo quarto simplici.

Longit. $2 \frac{1}{4}-2 \frac{1}{2}$ lin. Latit. $\frac{1}{2}$ lin.

Molschulsliy, Bull. de ha soe. de Moseou 1845. p. 356. 32.

IIabitat in insula sithha sub ligno et cortice nuper caesis non infreqquens; i) 1). Pippingsiöld, Frankenhaeuser et Holmberg.

In serie cum $\mathrm{S}_{\mathrm{t}}$. SPecchatore, provido etc. collocandus; a congeneribus punctura fortiore minus creberrima et thorace in medio magis dilatato diversus.

Mas: multo gracilior, abdominis segmento inferiore quinto late impresso sextoque emarginatis. ("Mälilinn)

58. Stexus a Dspector Mälilin: niger, creberrime punctatus, parce pubescens, palpis fuscis, basi testaceis: 


\section{$35 \quad 317$}

fronte leviter excavata, bisulcata, interstitio subcarinato; thorace latitudine vix longiore, obsoletissime canaliculato, lateribus modice rotundato; elytris thorace dimidio fere longioribus, inaequalibus, anterius prope suturam subtorulosis; abdomine nitido, subtilius punctato; femoribus ultra medium rufo-piceis.

Longit. 2 lin. Latit. parum ultra $\frac{1}{5}$ lin.

Ex insula Sitkha a 1). Frankenhaeuser transmissus; cum praecedente rarissime occurrit. Stexo scrutaton Erichson certe proximus, sed magnitudine paulo minore clytrisque adhuc longioribus diversum esse videtur. ("Mäklinn)

59. Stexus Paralegopipede Motschulsky: plumbeoniger, creberrime punctatus, palpis articulo primo testaceo, pedibus piceis, femoribus dilutioribus, piceorufis; fronte obsolete bisulcata, interstitio perparum convexo; thorace latitudine parum longiore, posterius angustato, sub-inaequali; elytris thorace longioribus, inter puncturam rugulosis.

Longit. $1 \frac{1}{5}$ lin. Latit. $\frac{1}{3}$ lin.

Habitat in insula Sitkha sub cortice arborum rarissime; D. Pippingsköld.

In vicinitate Steni husmis Erichs. ponendus. ("Mäklin ")

60. Stenus carriceps Mälilin: cylindricus, robustus, niger, nitidus, palpis fuscis, basi testaceis, parce fortiter punctatus; capite per totam longitudinem carinato, utrinque late sulcato; thorace latitudine tertia parte longiore, basin versus subtiliter canaliculato; elytris thorace sub-brevioribus, abdonine immarginato, apicem versus vix angustato, parcissime subtiliter punctato; tarsis articulo quarto simplici. 


\section{ric 36}

Longit. $1 \frac{2}{3}$ lin. Latit. fere $\frac{1}{2}$ lin.

Habitat in insula Sitkha rarissime; D. Frankenhacuser ("Mäklin")

61. Stexus brevipexnis Mäklin: niger, nitidus, fortius punctatus, antennis clava excepta, palpis pedibusque testaceis; capite elytris vix angustiore, fronte utrinque longitudinaliter sulcato-impressa, interstitio elevato, subcarinato; thorace latitudine paulo longiore, ante medium parum rotundato-dilatato, supra latius profundiusque canaliculato; elytris thorace brevioribus, sub-inaequalibus; abdomine subtilius parce punctato.

Longit. $1 \frac{1}{3}$ lin. Latit: fere $\frac{1}{3}$ lin.

In insula Sitkha a DD. Frankenhacuser et Holmberg locis humidis inventus.

Mas: abdominis segmento inferiore sexto apice emarginato, corpore toto magis angusto.

Femina: corpore robustiore, segmento laudato apice integro.

A Sten. flavifedi el pallipedi, quibus statura corporis pedumque structura proximus est, facillime dignoscitur elytrorum brevitate nee non magnitudine multo minore. ("Mäklinn)

62. Buenus longrexnes Mäklin: niger, densius fuscopubescens, tibiis posticis basi apiceque piceo-testaceis; thorace subtilissime coriaceo, subtiliter canaliculato, olsoletius parce punctato; elytris thorace plus quam dimidio longioribus, creberrime subtiliter punctatis.

Longit. $1 \frac{2}{5}$ lin. Latit. $\frac{1}{5}-\frac{1}{2}$ lin.

1). Pippingskïld unicum individuum in insula Sitkha cepit. ("Mäklin") 
63. PhLOE OrA EUS B I M P ES US Mälilin: niger, nitidus, antennis apicem versus, medio thorace, elytris pedibusque fuscis, antennarum basi, thoracis lateribus late, geniculis tarsisque rufo-testaceis; thorace minus dense subtiliusque punctato, ante basin foveis duabus transversis semicirculariter positis impresso, anterius obsolete canaliculato; elytris thorace duplo fere longioribus, creberrime punctatis; tibiis anticis integris, subtiliter ciliatis.

Longit. $1 \frac{1}{2}-1 \frac{2}{3}$ lin. Latit. $\frac{1}{2}$ lin.

Var. b: plus minusve rufo-picea, antennis, thorace, elytris pedibusque rufo-testaceis; elytris tamen interdum fuscis.

In insula Sitkha a DD. Frankienhacuser et IIolmberg locis humidis inventus; rarius occurrit. ("Mäklin")

64. Syrtomum ? coxfragosum Mäklin: subaeneonigrum, nitidum; antennis obcure rufo-piceis, articulo primo nigro; elytris aeneo-testaceis pedibus rufoferrugineis; thorace valde inaequali, confertissime sat fortiter at minus profunde punctato, postice carina longitudinali in medio evanescente tuberculisque duobus utrinque elongatis; elytris thorace dimidio fere longioribus, inaequalibus, confertim sat profunde et fortiter punctatis, interstitiis transversim oblique subrugulosis.

Longit. vix ultra 1 lin. Latit. $\frac{2}{3}$ lin.

In insula Sitkha a DD. Frankentaeuser et Holmberg excipulo e gramine rarissime inventum.

Fortasse proprii generis, Srnt. aeneo Müller vario modo dissimile. ("Mäklin")

65. Axтиорнaus aticolis Mannerheim. Bullet. de la Soc. de Moscou 1843. p. 234. 124. 
Var, b. macula elytrorum rufa.

In insula Sitkha a D. Frankenhaeuser semel inventa.

66. Arpedum ? в Revicolde Mäklin: elongatum, nigrum, nitidum, crebre et profunde punctatum, parcius pubescens; thorace longitudine duplo fere latiore, obsolete canaliculato, angulis posticis rotundatis, obtusis, margine basali sub-elevato; elytris thorace triplo fere longioribus, picescentilus; pedibus rufo-testaceis.

Longit. $1 \frac{1}{2}-1 \frac{3}{4}$ lin. Latit. $\frac{1}{2}$ lin.

In insula Sitkha excipulo e gramine a D. Frankenhaeuser rarissime inventum. ("Mäklin")

67. LatrRina i subcostatum Mälilin: rufo-testaceum, subnitidum, elytris dilutius testaceis, crebre profundeque punctatum, antennis apicem versus, capite, thoracis disco obsoletius, fasciisque duabus elytrorum obliquis, anteriore angusta saepe obsoleta, posteriore versus apicem lata fuscis, thorace inaequali canalicula profunda costis duabus postice abbreviatis inclusa impresso; elytris minus ordinate dense punctato-striatis, interstitiis quibusdam latioribus, subcostatis.

Longit. $1 \frac{3}{4}-2$ lin. Latit. $\frac{3}{4}-\frac{12}{5}$ lin.

In insula Sitkha a DD. Pippingsliöld, Frankenhaeuser et Ilolmberg locis humidis et sub ligno nuper caeso sat frequenter captum. ("Mäklin")

68. (DALIU STR I I PENe Mäklin: nigrum, nitidulum, antennarum basi pedibusque rufo-testaceis, thoracis lateribus elytrisque castaneis; illo sat crebre et profunde punctato, dorso foveolis duabus obsoletissimis notato: his thorace duplo fere longioribus, et crebrius 
et profundius punctatis, sub-rugulosis, longitudinaliter strigulosis.

Var.b. thorace obscure tesiaceo, limbo pallidiore, elytris pallidius testaceis.

Longit. $1 \frac{1}{2}-1 \frac{2}{3}$ lin. Latit. $\frac{2}{3}-\frac{3}{4}$ lin.

Habitat in insula silliha in graminosis frequens; 1)D. Frankenhaeuser et Holmberg. ("Maiklin")

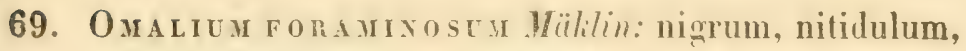
crebre punctatum, antenuarum basi anoque rufo-piceis, pedibus rufo-testaceis; vertice punctis duobus profundis impresso; thorace elytrorum basi parum angustiore, dorso foreolis duabus oblongis profunde impresso, lateribusque longitudinaliter subfoveolatis; elytris thorace parum plus quam duplo longioribus.

$\operatorname{Var}$ b. elytris piceis.

Longit. $1-1 \frac{1}{3}$ lin. Latit. $\frac{1}{3}-\frac{1}{2}$ lin.

Habitat in insula Sitkha in graminosis et sub cortice truncorum pini vulgaris: DD. Lippingsküld, Frankenhaeuser et Holmberg.

Magnitudine valde varians. ("Mäklin")

70. Omalie exscuptra Mälilin: breviusculum, nigrum, nitidulum; antennarum articulo secundo, thoracis lateribus elytrisque dilute piceis, pedibus testaceis; capite thoraceque parcius sed profunde punctatis, hoc foveis duabus latis inaequalibus, crebre punctatis exsculpto; elytris thorace dimidio latioribus et plus quam duplo longioribus profunde -, in medio subseriatim punctatis.

Longit. $1 \frac{1}{4}$ lin. Latit. fere $\frac{1}{2}$ lin. 
Habitat in insula Sitkha sub cortice pini rarius; DJ). Pippingsküld, Frankenhaeuser et Holmberg. ("Mäklin")

71. Onalium hasicole Mälin: depressum, nigrum, nitidulum, parcius obsoleteque punctulatum; antennarum basi, thoracis lateribus late, elytris, ano pedibusque dilute rufo-testaceis; thorace elytrorum basi parum angustiore, dorso foveolis duabus oblongis impresso; elytris thorace duplo longioribus, margine exteriore et angulo apicali nigris.

Longit. vix ultra 1 lin. Latit. $\frac{1}{4}-\frac{1}{3} \cdot \operatorname{lin}$.

Variat interdum thorace toto piceo, lateribus dilutius testaceis.

In insula Sitkha; sub cortice arborum sat copiose a DI). Pippingsköld, Frankienhacuser ef Holmberg inventum.

Inter individua, quae a I). Holmberg transmissa sunt, occurrunt quaedam magnitudine paulo minore, praeserlim angustiora, elytris tolis piceis et nonnihil brevioribus, verisimile masculini sexus. ("Mäklin")

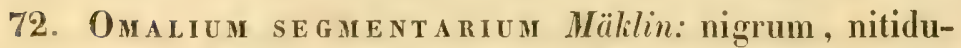
lam; antennis crassiusculis, apicem versus sensim adhuc magis incrassatis; thoracis lateribus, segmentorum abdominalium marginibus distincte anoque et pedibus rufo-testaceis; capite crebre punctato inter antennas bi - , in vertice unifoveolato; thorace subquadrato, aequaliter-punctato, ante basin canalicula brevi, longe ante medium abbreviata; elytris thorace dimidio longioribus, piceo-rufis, multo crebrius quam thorax punctatis, longitudinaliter substrigulosis.

Longit. $1 \frac{1}{2}$ lin. Latit. $\frac{1}{2}$ lin.

Individuum unicum in insula Sitkha a D. Holmberg inventum. 
Elytra ad angulum interiorem apice producta; verisimile: ad differentiam sexualem spectat. ("Mäklin")

73. Omalum roxguzum Mälin: lineare, elongatum, depressum, glabrum, nitidum, piceum; antennarum basi, thoracis lateribus, elytris, segmentorum abdominalium marginibus pedibusque rufis; antennis crassiusculis capite cum thorace multo brevioribus, apicem versus incrassatis; thorace subquadrato, basin versus leviter angustato, crebre punctato, postice vestigio canaliculae abbreviatae notato; elytris thorace duplo fere longioribus, crebre sat profunde punctatis, longitudinaliter sub-strigulosis.

Variat antennis totis thoraceque rufis.

Longit. $1 \frac{3}{4}$ lin. Latit. $\frac{1}{2}$ lin.

In insula Sitkha a DD. Frankenhaeuser et Holmberg excipulo locis graminosis rarius captum.

OM. Lineari Zett. Erichs. proximum. ("Mäklin")

74. Oмalium calcosum Mäklin: nigrum, nitidulum, confertim subtiliter punctatum, pube longiore densius vestitum; antennarum basi, thoracis lateribus anguste, pedibus elytrisque rufo-testaceis; his apicem versus infuscatis callo humerali sat evidenter elevato; thorace paulo ante scutellum tuberculo obsoleto laevigato notato.

Longit. $1 \frac{1}{4}$ lin. Latit. $\frac{1}{2}$ lin.

IIabitat in insula Sitkha rarissime; D. Frankenhaeuser. ("Mäklin")

75. Proteinus lim a tus Mällin: ovatus, niger, nitidulus; antennarum basi, thoracis limbo omni pedibusque 


\section{$8+i c$}

dilute piceo-testaceis; thorace elytrorum crebre punctulatorum basi parum angustiore.

$V a r$. b. thorace fere toto nigro, vix piceo marginato.

Var. c. thorace toto rufo-testaceo, elytris aut nigris aut rufo-testaceis plus minusve picescentibus.

Longit. $\frac{1}{2}-\frac{2}{3}$ lin. Latit. $\frac{1}{3}-\frac{2}{5}$ lin.

In insula Sitkha; a DD. Pippingskïld, Frankenhaeuser et Holmberg in fungis putrescentibus copiose lectus. ("Mäklin")

76. Protervis basalis Mäklin: ovatus, niger, nitidulus; antennarum et elytrorum basi late, apice vero anguste pedibusque testaceis; thorace elytrorum basi dimidio angustiore, lateribus plerumque testaceo marginato; elytris thorace plus duplo longioribus, crebre punctulatis.

Longit. $\frac{1}{2}-\frac{2}{3}$ lin. Latit. $\frac{1}{3}-\frac{2}{5}$ lin.

In insula Sitkha a DD. Frankienhacuser et Holmberg in fungis putridis rarius inventus.

Colore elytrorum et thorace angustiore a ceteris speciebus hujus generis facile dignoscendus. ("Mäklin")

77. Megartirus pictus Motschulsky: fusco-niger, opacus, crebre sat fortiter punctatus; antennarum articulis duobus primis, thoracis lateribus, elytrorum margine laterali et apicali anguste, abdomine pedibusque obscure-rufis; elytris basi late inaequaliter sordide testaceis maculam nigram suturam versus includentibus; thorace margine inaequali, subdentato, angulis posticis emarginatis. 
Longit. $1 \frac{1}{4}$ lin. Latit. $\frac{2}{3}-\frac{5}{14}$ lin.

Motschulsky, Bullet. de la Soc. de IIoscou 1845. p. 39. 101.

In insula Sitkha locis humidis sub lignis rejectis a DD.

Frankenhacuser et Ilolmberg inventus. ("Mälilin")

78. Megartir us atratus Mälilin: niger, sub-nitidus, creberrime punctatus; geniculis, tibiis tarsisque rufis; elytris prope suturam praesertim basin versus longitudinaliter impressis, apice anguste piceis; thorace lateribus rolundato, angulis posticis oblique truncatis.

Longit. $1 \frac{1}{3}$ lin. Latit. fere $\frac{2}{3}$ lin.

Habitat in insula Sithha; excipulo a D. Frankenhaeuser saepius captus.

Meg. dernesso Payk. Erichs. duplo fere major, angulis thoracis posticis vix emarginatis, sed latius oblique truncatis ut et colore elytrorum diversus. ("Mällin.)

79. Megartirus avgulicolis Mäklin: niger, subnitidus, creberrime punctatus; antennis piceis, pedibus brunneo-rufis; thorace profunde canaliculato, lateribus angulato.

Longit. 1 lin. Latit, fere $\frac{1}{2}$ lin.

In insula Sitkha locis humidis sub lignis rejectis a DD. Frankenhaeuser et Holmberg inventus.

Meg. sinuatocolli Dej. Erichs. perquam similis et affinis, sed minus opacus, thorace elytrisque adhuc fortius punctatis, abdomine e contrario nonnihil parcius punctato. ("Mäklin")

80. Micropeplus costatus Mäklin: niger, antennis rufo-brunneis; harum capitulo, thoracis lateribus pedibusque piceis; elytris convexioribus, apice de- 
pressis, dorso quadricostatis, costa tertia sive marginali proxima basi apiceque evanescenti; interstitiis elytrorum latioribus grosse punctatis.

Longit. vix ultra 1 lin. Latit. $\frac{1}{2}$ lin.

In insula Sitkha; in lignis rejectis a DD. Frankenhaeuser et Holmberg rarius inventus.

MItcr. poncato paulo major, nonnihil robustior, elytris convexioribus interstitiisque eorum latioribus, fortius sed minus dense punctatis, abdomineque minus profunde foveolato diversus.

Variat praeterea thoracis lateribus plus minusve rufopiceis. ("Mäklin")

81. Migropeples bruneus Mäklin: dilute rufo-brunneus, opacus, capite nigro; elytris thorace plus quam dimidio longioribus, sutura costisque tribus dorsalibus elevatis, interstitiis laevibus.

\section{Longit. $\frac{3}{4}$ lin. Latit. fere $\frac{1}{2}$ lin.}

Ilabitat in insula Sitkha in fungis arboreis rarissime; D Pippingsköld.

Interstitiis elytrorum laevibus cum Micr. tesserula Curtis, Erichs. convenit, sed nonnihil major elytrisque longioribus ut et colore diversus. ("Mäklin n)

82. Melanophila appendiculata Fabricius: subdepressa, atra, immaculata; thorace antrorsum subdilatato, basi apiceque bisinuato, medio distincte per totam longitudinem canaliculato, canalicula in foveola antescutellari incipiente, angulis posticis rectis nonnihil plicatis; elytris creberrime granulatis, supra subinaequalibus, apice acuminatis subtiliter serratis.

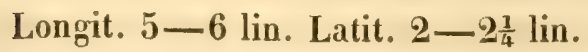


Mannerh. Bullet. de la Soc de Moscon 1837. VIII. p. 70. 5.

Apatura appendicheata Gory el Laporte, Monogr. des Bupr. (Apatura) p. S. Tab. II. fig. 14.

Bupnestis Oxyptenis) appendiclita, Kirby in Richardson Fauna Boreali-Americ. IV. p. 160. 214.

Punenops appendiculata, Dejean Cat. 3-ime édit. p. 89.

Bupnestis appendicclats, Schönherr Syn. Ins. III. p. 237. 126.

Buprestis morio, Payliull Faun. Svec. II. p. 230. 17.

Habitat in insula Sitkha; in domo a D. Pippingsküld, excipulo in herbis a D. Frankenhaeuser capta.

83. Epipinanis cornutus Eschscholtz.

Mas Mannerh. Bullet. de la Soc. de Moscou 1843. p. 238.134.

Longit. $2-2 \frac{1}{4}$ lin. Latit. $\frac{1}{2}-\frac{2}{3}$ lin.

Femina triplo major, posterius minus angustata, antennis ratione magnitudinis paullo brevioribus, praeterea vero ut in mare omnino constructis.

Longit. $3 \frac{1}{2}$. lin. Latit. $1 \frac{1}{6}$ lin.

Specimina jam quatuor hujus insecti pretiosissimi obtinui, quorum unum a D. Ferd. Sahllery, tria a D. Frantenhaeuser. Rarius occurrit in insula Sitkha secundum 1). Frankenhaeuser; interdum in domis, interdum in silvis excipulo captus. Omnia invidua, quae videre mihi contigit, colore nigro-piceo gaudent, antemis, pedibus elytrorumque basi plus minusve rufis.

84. Cryptonypus limbatus: niger, remote punctitus, longe et parce griseo-pubescens; thorace latitudine longiore, medio vix dilatato, obsoletissime canaliculato, angulis posticis extrorsum valde prominulis 
acutissimis; elytris striatis, horum limbo basali et marginali, antennarum articulo primo, thoracis angulis posticis pedibusque rufis.

Longit. $2 \frac{1}{2}$ lin. Latit. 1 lin. $=e$. Kactiol

Habitat in continenti Americes borealis ad fontes Kaknu;

D. Frankenhaeuser.

85. C O griseo-pubescens, punctulatus; thorace oblongo, lateribus inaequalibus tenue marginatis, angulis posticis nonnihil divaricatis, carinatis, apice incurvis truncatis; elytris striatis, eorum basi obsolete, margine vero inflexo pedibusque distincte rufo-castaneis.

Longit. 9 lin. Latit. $2 \frac{2}{3}$ lin.

Ilabitat in insula Sitkha sub cortice arborum emortuarum rarissime; D. Pippingsköld.

86. Dolopids selatus: elongatus, fusco-piceus, tenue pubescens, thoracis angulis posticis, antennis pedibusque ferrugineo-testaceis; thorace oblongo, crebre punctato; elytris punctato-striatis, pallide testaceis, macula longitudinali media communi alteraque utrinque laterali elongata nigro-fuscis.

Longit. $2 \frac{1}{2}$ lin. Latit. $\frac{5}{4}$ lin.

Habitat in insula Sitkha; in volatu a D. Holmberg semel captus.

87. Tinanasius a b ominalis Kirby: elongatus, niger, pubescens, abdomine testaceo, antennis pedibusque rufis; thorace subtiliter punctulato, apice angulatim impresso, medio canaliculato, basi marginato; elytris antice profunde punctato-striatis, fasciis duabus trans- 
versis, prima angustissima, valde flexuosa, extus dilatata, secunda lata extrorsum attenuata postice emarginata, cinereo-pilosis.

Longit. $3 \frac{1}{2}$ lin. Latit. $1 \frac{1}{4}$ lin.

Richardson, Faun. Bor. Amer. IV. p. 24\%. 333. Tab. II. fig. 5. - Klug, Berlin. Verhandl. 18'2. p. 308. - Spinola Monogr. Cler. Tab. XV. fig. 1. - Nomencl. of the Col. Ins. in the Coll. of Brit. Mus. IV. - Clerid. p. 15.

Thaxasumes pictes Spinola Monogr. Cler. I. p 19 '.

Habitat in insula Sitkha rarissime; D. Holmberg.

88. Scyduexus Califoricus Motschulsliy: niger, nitidus, parcius pilosus, ore, antennis, thoracis margine antico anguste, elytris pedibusque rulo-testaceis; vertice profunde et late excavato; thorace subquadrato, latitudine paulo longiore, basin versus nomnihil angustato lateribusque subsinuato, postice ante scutellum obsoletius impresso utrinque ad angulos foveolato; elytris parce obsoleteque punctulatis, apice subtruncatis.

Longit. $\frac{4}{5}$ lin. Latit. $\frac{7}{3}$ lin.

Motschulskin Bull. de la Soc. de Moscou 184\%. I. p. 48. 128.

In insula Sitkha a D. Frankenhaeuser semel excipulo inventus; D. Motschulsliy 1. c. variationem dilutiorem e California allatam descripsisse videtur.

Ad stirpem secundam operis "Nachträge zur Monographie der Gattung Scydmacmus von II. Schaum" in "Zeitschrift für die Entomologie, herausgegeben von E. F. Germar "Tom. V referendus. Scrdmaxo (Ermicro Lap.) tansato Müll. et Kunze, ut jam observavit V. Cl. Molschulsky, facie externa quodammodo similis, sed paulo minor, praesertim angustior. ("Mäklinw) 
89. SCY DMA N US B I F ORM M Mälilin: rufus, nitidus, parce griseo-pilosus, pedibus antennisque dilutius rufotestaceis; harum articulis quatuor ultimis evidenter crassioribus; capite angusto, collari valde elongato supra late impresso et dense piloso a thorace sejuncto; hoc elongato, latitudine dimidio fere longiore, apicem versus nomihil angustato, lateribus postice sinuato, ante basin transversim impresso, impressione carinula angustissima divisa et ad angulos basales utrinque foveolato; elytris laevibus, apice obtuse rotundatis.

Mas: antemnis apicem versus fortius incrassatis, articulo quinto latere externo producto, duobus insequentibus multo latiore.

Longit. vix ultra 1 lin. Latit. fere $\frac{1}{2}$ lin.

Marem in insula Sitkha D. Pippingsköld invenit sub lapide, feminas duo ibidem excipulo in gramine collegit D. Frankenhaeuser.

Ad stirpem quartam operis citati referenda est haec species. Scrdm. clavipedr $\boldsymbol{S} a y, S c h a u m$ certe affinis, sed thorace non griseo-hirto, colore etc. diversus. ("Mäklin")

90. Eutieia scitula Mäklin: nigra, nitida, antennis, pedibus elytrisque testaceis; thorace elytrisque obsoletius punctulatis, illo subquadrato, subtiliter canaliculato, postice depresso, utrinque bifoveolato, his truncatis.

Longit. $\frac{2}{3}$ lin. Latit. $\frac{7}{5}$ lin.

A D. Holmberg in insula Sitkha semel capta.

Euth. plicatae Gyll. (truncateldae Erichs.) similis et affinis, sed paulo minor, praesertim angustior. ("Mäklin") 
91. Necropiores grtevea Motschulsliy: niger, supra glaber; thorace undique crebre punctato, posterius angustato; elytris oblongo-quadratis, subtiliter punctatis, macula subhumerali andantiaca utrinque in margine inflexo; clava antemnarum dimidiatim testacea: pectore dense thavesenti-piloso; tibiis posticis recelis.

Longit. 8 lin. Latit. $3 \frac{1}{2}$ lin.

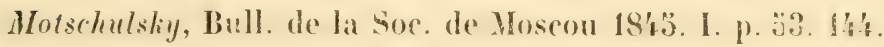

Necrophonts arribosts? Eschscholla, Dejean Cät. 3-ime édit. p. 131. sec. D. Motschulsivy, Bullet. de la Soc. de Moscou 1830. III. p. 3338 .

Ifabilat in insula sitkha, teste I). Molschulsky, qui specimen e Juseo suo ad describendum benevole transmisit.

92. Silpia Lippoxica herbst: subquadrata, depressa, nigra; capite, thorace scutelloque e pilis longis incumbentibus griseis sericeo-tomentosis, nigro-maculatis; elytris trilineatis, interstitiis tuberculis elevatis, magnitudine inaequalibus, seriatis, illis maris apice leviter sinuatis, feminae obtuse caudato-productis.

Longit. $5-5 \frac{1}{2}$ lin. Latit. $3-3 \frac{1}{3}$ lin.

Herbst, Col. V. p. 209. 33. Tab. 52. fig. 4. Fabr. Syst. EI. I. p. 338. 11. Gyllenh. Ins. Svec. I. p. 273. 12.

Orceptom Thustropules Lapposecen, hirby in Richardson Faun. Bor. Amer. IV. p. 100. 142.

Silpha caudati Say, Journ. Acad. Phil. III. I. p, 192. 1.

Habitat in continenti Imerices borealis, ad fontes Kakmu D. Frankenhacuser; ad sinum Kenai D. Holmberg.

93. Necropulos uates Eschscholla: suborliculatus, nigro-piceus, thoracis lateribus angulisque posticis 
late, elytrorum margine explanato tarsisque rufis; thorace transverso, antrorsum angustato-rotundato, basi et lateribus crebrius punctato; elytris profunde crenato-striatis.

Vur. b. tola plus minusve ferrugineo - vel rufotestacea.

Longit. $1 \frac{3}{4}-2$ lin. Latit. $1-1 \frac{1}{5}$ lin.

Agrrtes latrs Eschscholtz, Dej. Cat. 3-ème édit. p. 133.

Habitat in insula Sitkha, in ligno putrido sat frequens; DD. Frankenhaeuser et Pippingsköld.

Necnophlo glazro (Тнтома m. Payk. Gyll.) affinis, sed plerumque dimidio minor, praeterea brevior, antennis extrorsum multo tenuioribus, thorace punctato, elytris late explanato-marginatis, ut et colore bene distinetus.

94. Catops Frankexhauseri: elongatus, fusco-piceus, griseo-pubescens; antennis pectinatis, basi ferrugineis, articulis 4-10 globosis, spinam longam e singulo extus emittentibus, ultimo pyriformi apice acuminato; thorace quadrato, angulis rotundatis, obsolete canaliculato, postice in medio impresso; elytris oblongoellipticis, subtilissime punctulatis, tenue striatis, stria suturali profundiore, rufo-testaceis, cinereo-holosericeis, pilis longis fuscis praesertim in margine obsitis; pedibus ferrugineo-piceis.

Longit. $2 \frac{1}{2}-3$ lin. Latit. $1 \frac{1}{4}-1 \frac{1}{2}$ lin.

IIabitat in insula Sitkha; D. Frankenhaeuser. Specimina aliquot in cadavere humano in silva jacente et in fungis putridis capta.

Insectum imprimis antennarum forma insigne, quare proprium genus constituere liceat, si examini ulteriori subjiciatur. 
95. Catops Cryptopia gordes: oblongo-ovatus, convexus rufo-ferrugineus, nitidus, glaberrimus; antennis extrorsum valde incrassatis pilosis, articulo octavo praecedente multo minore; thorace laevi, antrorsum rotundato, angulis posticis supra elytra rotundatoproductis; elytris disperse punctatis, subrugulosis.

Longit. ${ }_{5}^{2}$ lin. Latit. $\frac{1}{3}$ lin.

In insula Sitkha sub lapide legit D. Pippingstöld.

Statura generi CoLovis magis vicinus, sed antennarum structura ad Catopes potius revocandus, quanvis glabritie. corporis ab ambobus alienus.

96. Colon inerus: oblongo-ovatus, convexus, fuscocastaneus, fulvo-pubescens, subtilissime crebre punctulatus; antennis brevibus, clava crassa arcuata; thorace antrorsum valde rotundato-angustato, basi truncato, angulis posticis subrectis; elytris stria suturali arcuatim valde impressa; pedilus muticis.

Longit. 1 lin. Latit. $\frac{7}{12}$ lin.

Habitat in insula Sitkha rarissime; D. Holmberg.

97. Peltis Pippingsköldir: oblongo-rotundata, subdepressa, rufo-picea, tenue griseo-pubescens; thorace brevissimo, crebre punctato, antrorsum valde angustato, pro capitis receptione profunde emarginato, basi truncato; elytris profunde striato-punctatis, interstitiis alternatim elevatis subcostatis, margine laterali late explanato, maculis undulato-bifasciatis lineisque tribus utrinque prope basin ferrugineis.

Longit. $4 \frac{1}{3}$ lin. Latit. $2 \frac{3}{4}$ lin.

Variat colore interdum dilutius ferrugineo.

Habitat in insulae Sitkhae fungis arboreis rarius; D. Pip pingsköld. 
Antennae clava triarticulata, articulo ultimo majore breviler ovato.

Oculi duo laterales globosi.

Frons apice truncata.

Palpi articulo ultimo subcylindrico, apice rotundato.

Tibiae anticae muticae.

Tarsi quinque-articulati.

Abdomen segmentis quatuor anterioribus liberis, haud connatis.

Corpus oblongum depressiusculum; thorace lateribus late explanatis subvelatis, margine serrato; elytris subconvexis, margine ante medium oblique explanato, serrato, humeris antrorsum nonnihil productis.

98. Peltastica tebercelata: rufo-picea; capite transrersin impresso; thorace brevi transverso, apice profunde emarginato, basi bisinuato, medio tertia parte elevato, crebre punclato, ante scutellum bituberculato, lateribus late explanatis, extrorsum et angulis posticis rotundatis, profunde cribellatis, flavescenti-pellucidis; elytris profunde striato-punctatis, sutura elevata, interstitiis quatuor subcostatis, in costis tuberculis nigris nitidis oblongis numerosis, interjectis hine inde lineolis albis concinne ornatis, undique marginatis, margine antrorsum oblique latius explanato, profunde cribellato, flavescenti-diaphano.

Var. b. elytris maculis lunatis communibus duabus albidis, una magna ante medium antrorsum versa alteraque minula pone medium retrorsum currata.

Longit. $1 \frac{1}{2}-2$ lin. Latit. $\frac{?}{5}-1$ lin. 
IIabitat in insula Sitkha, sub cortice truncorum pini rarius; DD. Frankenhaeuser et Holmberg.

99. Ins Dejearir Kirby: elongata, depressa, subparallela, nigro-picea nitida; thorace remotius, clytris autem creberrime punctulatis; his basi maculisque in utroque 2 el 2 inter se oblique positis, albido-testaceis; antennarum basi tarsisque rufo-piceis.

Longit. $2 \frac{1}{4}$ lin. Latit. $\frac{3}{4}$ lin.

Richardson, Faun. Bor. Amer. IV. p. 107. |133. 'Tab. II. fig. 4.

Specimen unicum in insula Silkha a D. Holmberg inventum.

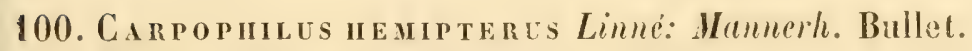
de la Soc. de Moscou 1843. p. 254. 174.

Erichson in Germar Zeitschrift. IV. p. 236. '. Naturg. Mer Ins. Deutschl. III. p. 133.2.

Var b. minor, elytris puncto humerali maculaque rotundata intra angulum interiorem apicis utrinque luteis.

Etiam in insula Sitkha inter allia allata individua viva Var. $a$ et $b$. invenit D. Holmberg.

101. Carpophizes dindatus Fabricills: oblongo-quadratus, nigro-piceus, antennis, pedibus elytrisque rufis; capite thoraceque profunde punctatis, hoc transversequadrato, angulis obtuse rotundatis; elytris minus profunde sed crebre punctatis, apice truncatis.

Longit. $1 \frac{1}{5}$ lin. Latit. $\frac{7}{12}$ lin.

Erichson in Germar Zeitschrift. IV. p. 239. 10.

Nitidula dimidita, Fabr. Ent Syst. I. 1. p. 261. 27. Syst. El. I. p. $33 \%$. 36. 
Ips pimbrata, Dejean, Cat. 3-ème édil. p 134.

Cum praecedente in insula Sitkha a D. Holmberg lectus.

"102. Cra ocepialus ? Unicolor Motschulsky: “ovatus, convexus, punctatissimus, fulvus, nitidus, palpis longissimis; thorace transverso, antice angustato, lateribus arcuatis; elytris crenulato-striatis; tibiis externe spinosis.") (Motschulsky.)

Longit. 1 lin. Latit. $\frac{2}{3}$ lin.

Motschulsky, Bullet. de la Soc. de Moscou 18/.5. IV p. 364.46 .

Habitat in insula Sitkha, sec. D. Motschulsky.

103. EPURAEA A U M R A T A: oblongo-ovata, subconvexa, punctatissima; thorace antice leviter emarginato, lateribus praesertim basi late reflexo-marginato, angulis posterioribus rotundatis; elytris apice subtruncatis, angulis ibique rotundatis; fusco-testacea, thorace elytrisque margine rufo-ferrugineo, pedibus interdum pallidioribus.

Longit. $1 \frac{1}{4}-1 \frac{2}{3}$ lin. Latit. $\frac{1}{2}-\frac{3}{4}$ lin.

Habitat in insula Sitkha, sub cortice truncorum pini non infrequens; DD. Frankenhaeuser et Holmberg.

Statura fere Nitidulae (Epuraeae) convexiusculae Mannerh. (Bull. de la Soc. de Moscou 1843. p. 255. 176.) sed paullo longior, minus convexa, crebrius punctata et aliter colorata.

104. Epuraea truncatelda Mannerheim.

Nitidula truncatela Mannerh. Bullet. de la Soc. de Moscou 1846. II. p. 514. 12. 
$V$ ar. b. obscure nigro-fusca, antennarum basi, tibiis thoracis limbo late elytrorumque margine angustius rufoferrugineis.

Epuraea atrata Motschulsky in litteris.

IIabitat in insula Sitkha, sub cortice truncorum pini frequens; DD. Frankenhaeuser, Pippingsköld et Holmbery.

105. Epuraea plandata Eschscholla: oblonga, depressa, ferruginea, opaca, tenuiter pubescens; antennarum articulo ultimo fusco; thorace apice leviter emarginato, crebre punctulato, lateribus late reflexomarginato, angulis posticis rectis; elytris dense punctatis, apice subtruncatis.

Var. b. luteo-castanea, oculis nigris.

Longit. $1-1 \frac{1}{2}$ lin. Latit. $\frac{1}{3}-\frac{1}{2}$ lin.

Erichson, in Germar Zeitschrift. IV. p. 271. 19.

Habitat in insula Sitkha, D. Eschscholtz; sub cortice truncorum pini a DD. Pippingsköld et Frankenhaeuser capta; minus frequenter occurrit.

Praecedenti affinis, sed magis depressa, thorace elytrisque brevioribus, thoracis margine laterali magis reflexo et elytris apice minus truncatis diversa.

106. Criptopiagus octodentatus Mälilin: oblongus, subdepressus, ferrugineus, dense punctatus, pube longiore dense vestitus, thoracis margine antico elytrisque testaceis; thorace bituberculato, lateribus utrinque quadridentatis.

Longit. $1 \frac{1}{3}$ lin. Latit. $\frac{1}{2}$ lin.

In insula Sitkha in fungis a DO. Pippingsköld et Holmberg inventus.

Vix vestigium tuberculorum thoracis basalium observandum. ("Mäklin") 
107. Cruptoriager tuberculoses Mäklin: oblongoovatus, testaceo-ferrugineus, subtilius crebre punctatus, pube subtili densius vestitus; thorace evidenter bituberculato, lateribus obtuse bidentatis, margine posterius obsolete crenulato.

Longit. $1 \frac{1}{5}$ lin. Latit. fere $\frac{1}{2}$ lin.

Ilabitat in insula Sitkha rarissime; I). Frentenhacuser.

Versus marginem basalem thoracis tubercula duo alia obsoleta observantur; anteriora parva quidem, sed distincte elevata. ("Mälilinn)

108. Atomania fuscicoldis: elongala, leviler convexa, nigro-picea, vix nitida, longe griseo-pubescens, profuncle remotius punctata; antennis crassis ut el genubus tibiisque rufis; thorace antrorsum angusiato, postice obsolete carinato, lateribus subinaequali; ely tris anterius confuse strigulosis; antennis approximatis.

Longit. $\frac{3}{4}$ lin. Latit. $\frac{1}{4}$ lin.

Cryptophagus Canfonweus Motschulsky in litteris.

Ilabitat in insula Silkha; in volat!ı a D. Pippingslüld semel capta.

Ob nomen Cruptopulai Calfonsicr jam aliae speciei datum Mannerh. Bullet. de la Soc. de Mosenn. 18'3.3. p. 230 . 178.) denominationem (1. 1) Motschulskiy mutare debui.

109. A tomara gepidea Mälin: ovalis, leviter convexa, rufa, nitida, subtiliter cinereo-pubesens; thorace lateribus sub-rotundato profunde-, elytris subtiliter punctatis; antennis non approximatis.

Var. b. tota testacea.

Longit. 1 lin. Latit. fere $\frac{1}{2}$ lin. 
Ifabitat in insula Sitkha rarissime, a I). Ilolmberg semel tantum inventa; varietatis $b$. individua duo sub cortice pini capta transmisit D. Frankenhaeuser.

It. cogsatae Erichs. magniludine aequalis, sed latior. ("Mäklin")

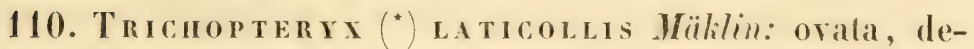
pressiuscula, nigra, subtilissime confertim punctulata, dense pubescens; thorace elytuis paulo latiore, apicem versus angustato, angulis posterioribus productis, deflexis, acutis; antennis fuscis, elytrorum margine postico anguste pedibusque rufis.

Longit. abdomine contracto vix ultra $\frac{1}{2}$ lin. Latit. fere $\frac{1}{3} \operatorname{lin}$.

D. Itolmberg hane speciem in insula sitkha rarissime invenit.

Tr. Атомив De Geer major, el ut V. Cl. Molschulsliy in lit. indicavit maxima omnium specierum cognitarum hujus generis. ("Miiklin")

111. Triciopterrx ixsularis Mälitil (**): oblongoquadrata, depressiuscula, nigra, subtilissime confertim punctulata, dense pubescens; antemis piceis, harum basi, elytrorum margine apicali pedibusque rufotestaceis; thorace elytrormu latitudine, apicem versus parum angustato, angulis posterioribus productis.

(*) V. CI. Iotschulsky pro denominatione Tucnopterux, antea usitata, nomen huic generi novum in Bull. de la Soc. de Hoscon 1848. p. 3669. Acrotrucus proposuit, quod tamen in continuatione operis in AcraTRICus commutavit. ("Mäklin»)

(“) Es ist dies die Art, von der ich Herrn r. Motschulsky ein Exemplar gezeigt habe, deren er in Bullet. de la Soc. de Moscou. 1831. III. p. 217. erwäht; er bestimmte mir dieselbe damals als Tr. serocus Schiippel und später bringl er sie zu seiner Acratrichs atrata, von welcher sie sicher verschieden ist, wenigstens wegen ihrer ganz rothen Beine. 
Longit. fere $\frac{8}{5}$ lin. Latit. $\frac{1}{5}$ lin.

In insula Sitkha a DD. Frankenhaeuser et Holmberg excipulo locis graminosis copiose collecta.

Magnitudine intermedia inter Tr. Grandicollem Märkel, Mannerh. Erichs. et Tr. Fascicularem Herbst. Erichs.; statura corporis magis elongata, elytris paulo longioribus et punctura subtiliore ab utraque diversa. ("Mäklin")

*112. Triciopter y X Sithä̈sis Motschulsky: “ovata, depressiuscula, nigra, subtilissime confertim punctulata, dense atro-pubescens; thorace subtriangulari, antice angustato, angulis posterioribus productis; elytris in medio subdilatatis, postice suboblique truncatis; antennis pedibusque piceis." (Motschulsky)

Longit. vix $\frac{1}{8}$ lin. Latit. vix $\frac{1}{5}$ lin.

Allibert in Guérin-Méneville Rev. Zool. par la Soc. Cuvier. 1847. p. 196. 20.

Prilicm Sitranse, Motschulsky Bullet. de la Soc. de Moscou 1845. p. 526. 5. Tab. X. fig. 13.

Acrotrichis Sitraensis, Motschulsky Bullet. de la Soc. de Moscou 1848. tab. ad pag. 568. N. 15.

Acratricuis Sitraensis, Mlotschulsky Bullet. de la Soc. de Moscou 1850. p. 237.

Acratricuis Sitransis, Motschulsky Bullet, de la Soc. de Moscou. 1851. III. p. 215. 17.

Habitat in insula Sitkha, sec. D. Motschulsky.

113. Ptenidiu pulum Mäklin: nigrum, nitidum, parce pilosellum; pedibus antennisque testaceis, harum clava nonnihil infuscata; elytris obsoletius punctulatis; thorace basi obsolete bifoveolato.

Longit. $\frac{1}{t}-\frac{1}{5}$ lin. Latit. $\frac{1}{7}$ lin. 
In insula Sitkha a DD. Pippingsköld et Holmberg inventum.

Pten. mymecophico Motsch. paulo majus, elytris apicen versus magis angustatis. ("Mäklin")

114. BYRRHUS CYCLOPHOR US Kirby.

Richardson, Faun. Bor. Amer. IV. p. 117.167.

Var. b. Mannerh. breviter ovalis, nigro-piceus, subtilissime coriaceus, castaneo-tomentosus; antennis articulo primo excepto rufis; elytris vittis duabus parum interruptis nigris, dorso macula media communi circulari obsoleta e pube cinerea.

Longit. $3 \frac{1}{4}$ lin. Latit. 2 lin.

IIabitat in continenti Americes borealis, ad fontes Kaknu;

D. Frankenhaeuser.

115. Morycius a cumitus Motschulsky: ovatus, globosus, postice attenuatus, supra aeneo-cupreus, nitide resplendens, parce griseo-pubescens, remote punctatus, antennarum basi tarsisque rufo-ferrugineis.

Longit. $1 \frac{5}{11}-2$ lin. Latit. $1-1 \frac{1}{4}$ lin.

Habitat in insula Sitkha, sub lapidibus intra muscos sat frequens; DD. Frankenhaeuser et Pippingstiöld.

II. Aeneo Fabr. subsimilis, sed elytris posterius attenuatis, punctura multo remotiore et colore magis metallice splendido imprimis diversus.

116. Simplocaria nitida Motschulsky: oblongo-ovata, supra viridi-aenea, nitida, parce pilosa, disperse profunde punctata; capite convexo, fronte impressa subcanaliculata; thorace transverso, elevato-pulvinato; elytrorum margine reflexo et antennis pedibusque rufo-piceis, corpore subtus violaceo. 
Var. b. forsan immatura, elytrorum margine posterius late, corpore subtus toto, antennis pedibusque ferrugineo-testaceis.

Longit. $1 \frac{1}{3}-1 \frac{1}{2}$ lin. Latit. $\frac{2}{3}-\frac{3}{4}$ lin.

Motschulsky, Bullet. de la Soc. de Moseou 18'r5. IV. p. 362. 41 .

Ilabitat in insula Silkha, sub cortice arborum emortuarum rarius; DD. Frankenhacuser et Pippingsköld; sub ligno recenter caeso; 1). Holmberg.

117. Anpincyrt simplicipes Motschulsky: globosoovata, supra cupreo-aenea, nitida; capite parce punctato, fronte inaequali, in medio lineola impressa; thorace laevissimo, versus latera parce piloso, antrorsum angustato-compresso, angulis anticis productis lobatis; elytris disperse punctulatis, postice declivibus apice rotundatis; antennis, corpore subtus pedibusque piceo-testaceis; tibiis anticis inermibus.

V'ar.b. supra et subtus nigro-violacea, antennis pedibusque rufo-ferrugineis.

Longit. $1 \frac{3}{4}-2$ lin. Latit. $1 \frac{1}{3}-1 \frac{1}{2}$ lin.

Habitat in insula Sithha, sub cortice arborum emortuarum rarius; DD. Frankenhaeuser et Pippingsköld; sub ligno nuper caeso; D. Holmberg $\left.{ }^{*}\right)$.

(") Hier muss ich einen von mir friher gemachten gewaltigen Missgriff berichtigen, auf den Herr von Motschulsliy später meine Aufmerksamkeit lenkte. Ich stellte nämlich in meinem Beitrag zur KäferFauna der Aleutischen Inseln, der Insel Sitliha und Veu-Californiens (Bullet. de la Soc. de Moscou 1813.) p. 286. unter den Helopier eine neue frattung Eccrous auf, und beschrieb, p. 287, davon als neu eine Art, Irbosonones von mir genannt. Es hat sich aber seitdem erwiesen, dass der fragliche Käfer nichts anderes ist als die Ampuczera DExtipes Eschscholtz, von Dr. Steffahny in Germars Zeitschrift. IV. 


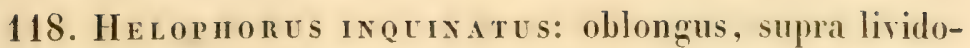
testaceus, glaber, hine inde virescenti-metallescens, subtus piceo-testaceus, dense griseo-pubescens, pedibus ferrugineo-testaceis; thorace lateribus subrectis, angulis obtusatis, supra rugoso-punctato, quinquesulcato, sulcis secunda et quarta extrorsum valde flexuosis; elytris maculis aliquot fuscis signatis, profunde punctato - striatis, interstitiis alternis elevatioribus.

Longit. $1 \frac{2}{5}$ lin. Latit. $\frac{3}{4}$ lin.

Habitat in insula Sitlha, locis humidis sub lapidibus rarius; D. Holmberg.

119. CER C X F F Y IPEXXE: subrotundatum, convexum, nigrum, nitidum, thoracis lateribus pedibusque rufoferrugineis, elytris fulvis, tenue punctato-striatis, interstitiis leviter punctulatis.

Longit. $1 \frac{1}{3}$ lin. Latit. 1 lin.

IIabilat in insulae Sitkhae stercore bovino rarius; $\mathbf{b}$. Holmberg.

C. minzito Mannerh. (Bullet. de la Soc. de Moscou. 1813. p. 260. 189.) latius, suborbiculatum, elytris remotius punctulatis, striis minus profunde exaratis ut et colore diversum.

p. 40. bereits charakterisirt. Vielleicht wiirde es mir doch zu irgend einer Entschuldigung gereichen, dass dieses gewiss in manchen Theilen paradoxe Thierchen von dem grossen Entomologen Dejean zu den Chrysomelinen gezogen, und ihm jetzt ron Dr. Steffahmy einen Platz unter den Byrrhiden angewiesen worden ist. Auch Entomologen ersten Ranges wie Gyllenhal und Dejean hatten die Prrnolom Fonssтпӧми mit den Carabicinen zusammengestellt, bis endlich der Scharfblick eines Erichsons sie unter die Silphalen einreihte. 
120. CERCYON F I B I A T M: ovatum, convexum, nigrum, opacum, creberrime punctulatum; thorace lateribus piceo-testaceis; elytris profunde striatis, subsulcatis, margine in medio anguste rufo-testaceo, striga intramarginali postica lineolisque tribus apicalibus plus minusve confluentibus pallide luteo-testaceis.

Var. b. thoracis margine latius piceo-testaceo; elytris margine toto, posterius introrsum dentato, luteo - testaceis.

Var. c. thorace piceo-testaceo, dorso infuscato; elytris margine basali, toto laterali ad apicem dilatato introrsum dentato, maculisque aliquot intramarginalibus testaceo-pallidis, femoribus piceo-testaceis.

$V a r . d$. nigra, thoracis margine laterali antice et postice dilatato rufo - ferrugineo; elytris livido - testaceis, macula postica utrinque obliqua nigra posterius bidenticulata.

Longit. $1 \frac{1}{8}-1 \frac{3}{4}$ lin. Latit. $\frac{5}{6}-1 \frac{1}{5}$ lin.

Sub fucis e mare rejectis in insula Edgecombe a D. Frankenhaeuser copiose lectum.

121. Cercy on post I CA TuM: subhemisphaericum, nigrum; thorace subtilissime remotius, elytris vero densissime punctulatis, his vix conspicue striatis, puncto humerali sanguineo, apice late rufo-testaceis; pedibus obscure rufo-piceis.

Var. b. thoracis lateribus elytrisque obscure rufopiceis, his apice nonnihil pallidioribus.

Longit. $\frac{2}{3}-\frac{3}{4}$ lin. Latit $\frac{1}{2}-\frac{2}{3} \operatorname{lin}$.

In insula Sitkha excipulo a D. Frankenhaeuser captum ; in stercore bovino D. Pippingsköld. 
C. Lugusm Payk, afline, sed brevius, magis rotundatum of thorace fere semper toto nigro diversum.

122. Anisotoma hateritia: breviter ovata, convexa, rufo - ferruginea, oculis clavaque antennarum nigro fuscis; thorace transverso, crebre punctato, angulis posticis subreclis; elytris profunde striato-punctatis, interstitiis confuse seriato-punctatis.

Longit. $1 \frac{2}{3}$ lin. Latit. 1 lin.

In volatu in insula Sitkha a D. Holmberg semel capta.

123. Heteropiaga Mauritanica Fabricius: oblonga, subparallela, supra nigro-picea subnitida, subtus cum antennis pedibusque obscure rufo-picea; thorace brevi transverso punctatissimo, lateribus posticeque marginato, basi bisinuato; elytris profunde striato - punctatis, interstitiis subconvexis, crebre punctatis.

Longit. $2 \frac{9}{3}$ lin. Latit. $1 \frac{1}{4}$ lin.

Dejean, Cat. 3-ème édit. p. 220.

Tenebrio Mauritanicus, Fabr. Ent. Syst. I. 1. p. 113. 15. Syst. El. I. p. 149. 27. Schünh. Syn. Ins. I. p. 152. 33.

Habitat in insula Sitkha; D. Ferd. Sahlberg: intra allia allata ibidem etiam cepit D. Holmberg.

124. CERANDRAA CORNUTA Fabricius: lineari-elongata, subdepressa, rufo-ferruginea; capitis laterum marginibus auriculatis valde dilatatis oculos nigros ambientibus; mandibulis porrectis magnis apice recurvis acutis corniformibus; vertice breviter bicornuto; thorace transversim quadrato, punctulato, margine postico utrinque foveolatim impresso; elytris tenue striatopunctatis, interstitiis subtilissime punctulatis. (Mas.) 
Femina differt mandibulis occultis, capite thoraceque angustioribus, illius margine reflexo leviter auriculato oculos ambiente, verticeque obsolete binodulo.

Longit. 2 lin. Latit. $\frac{3}{4}$ lin.

Dejean, Cat. 3-ème édit. p. 222.

Phaleria cornuta Latr. Gen. Crust. et Ins. II. p. 175. 1. Tab. X. figg. 4. 5.

Ulom conseta Stephens Illust. Mandib. 10. Manual of Brit. Col. p. 322. 2532.

Trogosita convuta Fabr. Ent. Syst. Suppl. p. 51. Syst. El. I. p. 1552 .

Ilabitat in insula Sitkha D. Ferd. Sahlberg; verisimile cum rebus mercatoriis importata.

125. Ceraxdra maxillosa Fabricius: lineari-elongata, subdepressa, rufo-ferruginea; capite minuto, lateribus parum producto, transversim profunde impresso excavato; vertice cornubus duobus minutis approximatis armato; oculis nigris lateralibus; mandibulis exsertis, elevatis, falcatis; thorace quadrato, punctulato, margine postico utrinque foveolatim obsolete impresso; elytris tenue striato-punctatis, striis punctisque remotioribus, interstitiis subtilissime punctulatis. (Mas.)

Femina differt capite omnino mutico, antice transversim modice impresso mandibulisque occultis.

Longit. $1 \frac{1}{2}$ lin. Latit. $\frac{1}{2}$ lin.

Dejean, Cat. 3-ème édit. p. 222.

Trogosita maxillosa Fabr. Syst. El. 1. p. 155. 25.

IIabitat in insula Sitkha; DD. Etholén et Ferd. Sahlberg: codem modo ac praecedens species introducta. 
126. Dircaea Holmbergit: elongata, fusco-picea, crebre et sat profunde punctata, tenue pubescens; antennarum tibiarumque basi ut et thoracis limbo ommi pallide testaceis; elytris fasciis tribus valde undulatis, postica magis dilata, maculisque duabus ante apicem, externa marginali longiore, albido - testaceis.

Longit. $2 \frac{1}{3}$ lin. Latit. $\frac{2}{3} \operatorname{lin}$.

Ifabitat in insula Sitlha rarissime; 1). Molmberg.

Speciei hujus elegantissimae unicum tantum specimen sub cortice arboris nuper caesae inventum.

127. Prtino Santuergin Mannerheim: Bullet. de la Soc. de Moscou. 1843. p. 285. 234.

Var. b. nigro-picea obscura, elytrorum humeris et margine pedibusque rufo-piceis.

Longit. $5 \frac{1}{\frac{1}{2}}$ lin. Latit. $1 \frac{\circ}{5}$ lin.

IIane speciem in ligno putrido pini uon infrequenter legit in insula Sitkha D. Frankenhacuser; varietas vero rarissime occurrit.

128. Tenebrio molt or Linne: lineari-elongatus, nigropiceus, subnitidus, subtiliter punctulatus, subtus rufopiceus; elytris obsolete striato-punctatis; femoribus anticis brevibus.

Longit. 7 lin. Latit. $2 \frac{1}{2}$ lin.

Linne, Syst. Nat. II 672. 2. Faun. Svec. 815. Gyllenh. Ins. Svec. II. p. 591. 1. Schönh. Syn. Ins. I. p. 148. 9.

In insulae Sitkhae domo a D. Frankenhaeuser semel captus: verisimile ex Europa vel Sibiria allatus.

129. Stenotracieles obscurus: elongatus, obscure nigro-aeneus, punctatissimus, tenue griseo-pubescens; 


\section{6}

thorace quadrato, lateribus pone medium leviter sinuato, angulis omnibus obtusis; elytris minus inaequalibus, subsulcatis; margine inflexo femorumque basi piceo-testaceis.

Longit. 6 lin. Latit. $1 \frac{3}{4}$ lin.

Habitat in continenti Americes borealis ad fontes Kaknu:

D. Frankenhaeuser.

St. aeneo $\bar{P}$ ayli. valde affinis, punctura omnino eadem, sed colore obscuriore, antennis totis nigris, thorace breviore quadrato elytrisque multo brevioribus minus inaequalibus, per totam longitudinem subsulcatis dignoscendus.

130. Pogonocerus Erinemeromes Ménétriés: elongatus, depressus, ferrugineo-testaceus, tenue pubescens; oculis magnis nigris; capite, thorace pedibusque rufotestaceis; antennis articulis $3-5$ serratis, $6-10$ rarum sensim longiorem emittentibus; thorace minuto laevi, basi marginato, antrorsum angustato, medio obsolete canaliculato; elytris praelongis, intra humeros utrinque impressis, apicem versus nonnihil dilatatis, apice ipso rotundatis, supra crebre punctatis, obsolete subcostatis.

Longit. 7 lin. Latit. $2 \frac{1}{3}$ lin.

Habitat in insula Sitkha rarissime; in domo a D. Pippingsköld semel inventus.

131. Araspis sericen Mannerheim: Bullet. de la Soc. de Moscou 1843. p. 288. 237.

Var. c. nigro-picea, holosericeo-pubescens, antennarum basi, tibiis tarsisque ferrugineo-testaceis.

Habitat in insulae Sitkhae floribus Heracleorum rarius; ceteras varietates iisdem locis copiose legit D. Frankenhaeuser. 
132. Meloë strigulosus: elongato-angustatus, ater, tarsorum unguiculis solis rufis; antennis articulis 5-7 dilatatis difformibus; capite thoraceque disperse punctulatis; hoc capite angustiore latitudine longiore, antrorsum lateribus rotundato, posterius parum angustato, basi profunde emarginato, supra obsolete canaliculato et ante basin longitudinaliter profunde impresso; elytris abdomineque subtiliter undulato-rugulosis. (Mas.)

Femina differt capite thoraceque paullo latioribus et antennis simplicibus.

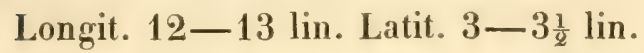

Habitat in insula Kodiak; D. Etholén; marem in California boreali captum dedit D. Cygnaeus.

\section{T A N Y R H I N US.}

Antennae in medio rostri insertae; articulis $1-4$ tenuibus; primo pyriformi duos insequentes simul sumtos longitudine aequante; secundo et quarto teretibus aequalis longitudinis; tertio nonnihil longiore; $5-10$ hirtis praecedentibus triplo latioribus, inter se aequalibus; ultimo oblongo decimo duplo longiore.

Rostrum capite paullo longius, planum, lateribus utrinque tenue sulcatum, inter oculos subcarinatum; mandibulae validae arcuatae; palpi articulo ultimo oblongo, subinflato, apice rotundato.

Caput elongato-quadratum; fronte profunde impressa; oculis subdepressis, vix prominulis.

Thorax elytris duplo angustior, latitudine postica vix longior, basi subtruncatus, angulis rectis, ante medium an- 
trorsum angustatus ibique latitudine postica plus quam duplo angustior.

Elytra thorace triplo longiora, humeris rotundatis parum elevatis, mox pone humeros dilatata, dein apicem usque linearia, dorso subconvexa, apice truncata angulo exteriore rotundato.

Pedes modice elongati; tarsi omnes distincte 5 -articulati, articulo primo insequente paullo longiore, $2-4$ aequalibus, ultimo longitudine tribus praecedentibus simul sumtis aequali, unguiculis acutis incurvis.

Genus valde paradoxum, pentamerum, sed ad Rhinosimida tamen referendum.

133. ThN YHIXUS SINGULA Is: nigro-piceus; capite thoraceque inaequalibus, profunde punctatis; elytris profunde punctato-striatis, striis versus apicem inter se connexis; antennarum basi pedibusque rufo-testaceis.

V $a r . b$. tota pallide testacea, forsan immatura.

Longit. cum rostro $2-2 \frac{1}{2}$ lin. Latit. $1-1 \frac{1}{4}$ lin.

In insulae Sitkhae silva excipulo unicum tantum specimen legit D. Frankenhacuser; Var. b. in volatu a D. Pippingsliöld capta.

13\%. SAlPINGU ELONGATUS: elongatus, testaceo-aeneus, glaber, nitidus; rostro brevissimo utrinque canaliculato, canaliculis in fronte conjunctis; thorace angustato, oblongo, rude et valde profunde punctato; elytris profunde striato-punctatis, singulo forea ante medium transversim impressa.

Longit. 2 lin. Latit. $\frac{1}{2}$ lin. 
Sub ligno nuper caeso in insula Sitkha rarissime occurrit;

D. Holmberg.

135. LIOPHL OEU I Q U I A T US: subovatus, nigro-piceus, parce flavescenti-pilosus, antennis pedibusque rufopiceis; rostro medio anguste carinato; thorace fortiter remotius punctato, lateribus ante medium modice rotundato, dorso e squamulis testaceis trilineato; elytris profunde striato-punctatis, interstitiis punctulatis, tertia interdum subelevata, dense testaceo-squamosis, maculis numerosis denudatis, vel nigro-piceis maculis numerosis testaceo-squamosis; femoribus muticis.

Longit. $3-3 \frac{1}{2}$ lin. Latit. $1 \frac{1}{4}-1 \frac{1}{2}$ lin.

IIabitat in insula Sitkha, sub lapidibus et intra muscos, sed in loco tantum unico ibique frequenter observavil D. Frankenhaeuser.

L. Trunsmarivo Schönh. sp. indescr. e California valde affinis, differt vero thoracis disco et elytrorum striis remotius profundiusque punctatis.

136. LePYRUS GEMELLUS Kirby: oblongo-ovatus, niger, pilis griseo-brunneis dense obtectus; rostro thoraceque in medio argute carinatis; hoc elytrisque rugulosogranulatis, granulis nitidis denudatis; thorace cinereobivittato; elytris striis gemellatis in utroque quinque postice inter se conjunctis, punctoque in connexu striarum secundae et tertiae minuto albidis, interstitiis alternis subelevatis.

$V a r . b$. elytris punctis minutissimis numerosis conspersis, et utrinque postice sub callo paullo majore. notatis albidis; cetera ut in $a$.

Longit. $6 \frac{2}{3}$ lin. Latit. $2 \frac{1}{8}$ lin. 
Richardson, Fauna Bor. Amer. IV. p. 198. 265̆. Tab. V. fig. 7. Schönh. Gen. et Spec. Curcul. VI. p. 297. 5.

IIabitat in continenti Americes borealis; ad fontes Kaknu;

D. Frankenhteuser; Var. b. e vicinitate sinus Kenai a

D. Holmberg transmissa.

137. Plinthus carivatus Eschscholtz: elongatus, niger vel nigro-piceus, parce silaceo-vel fulvescenti-squamosus; rostro rude striolato-rugoso; thorace oblongo, rude rugoso-punctato, antice alte carinato et utrinque impresso, lateribus obsolete crenato; elytris mediocriter striato-punctatis, sutura interstitiisque alternis elevatis carinatis, humeris fasciisque duabus versus medium conniventibus ut et maculis interjectis fulvosquamosis; femoribus tibiisque intus dentatis. (Mas.)

Longit. cum rostro $3-5$ lin. Latit. $1-1 \frac{3}{4}$ lin.

Dejean, Cat. 3-ème édit. p. 286. Schönh. Gen. et Spec. Curc. VI. p. 334. 20.

Femina: oblonga, nigra opaca, scrobiculata et verrucosa; fronte foveola impressa; thorace antice alte carinato, utrinque lacunoso, lateribus rotundatis, crenatis; elytris granulis minutis adspersis, interjectis punctis impressis hinc inde seriatis, verrucis forma inaequalibus, jam magnis oblongis, jam minoribus, undique obsitis, margine laterali serrato; thorace maculis duabus lateralibus, elytrisque utrinque humerali et in verrucis pustulis obsoletis e squamulis fulvescentibus; tibiis anticis biarcuatis muticis.

Longit. cum rostro $5 \frac{1}{2}-6$ lin. Latit. $2-2 \frac{1}{2}$ lin.

Meicipus scroniculatus ? Mannerh. Bullet. de la Soc. de Моscou 18'3. p. 292.217.

Habitat in insula Sitkha in cortice et ligno e pinis recenter caesis sat frequens; DD. Frankenhaeuser et Pippingshöld. 
138. Pissodes costatus: oblongus, minus convexus, nigro-piceus, sparsim albido-squamosus; rostro elongato tenui, apice nonnihil dilatato; thorace postice leviter sinuato, angulis subrectis, supra profunde punctato, longitudinaliter carinato, in medio foveola utrinque impressa; elytris profunde striato-punctatis, striis gemellatis, interjectis interstitiis tribus elevato-costatis; thoracis foveolis lineisque duabus posterius divergentibus luteo-vel albo-squamosis, scutello niveo, elytris ante medium maculis duabus oblique positis luteo-squamosis fasciaque pone medium a maculis tribus, quarum media luteo -, ceterae autem niveosquamosae.

Longit. cum rostro $3-4$ lin. Latit. $1-1 \frac{1}{2}$ lin.

Habitat in truncis pinorum recenter deciduarum et in cortice crescentium insulae Sitkhae minus frequens; DD. Frankenhaeuser et Holmberg.

P. Gyllenhali Schönh. (Hercyniae Gyllenh.) similis, ejusque forma fere omnino, sed thorace fortius punctato, argutius carinato, elytris costatis, postice distinctius callosis, ut et fascia maculari postica haud contigua abunde distinctus.

\section{E II P H Y A T ES.}

Antennae breviusculae, validae, funiculo 8 -articulato; articulo primo crasso, obconico; secundo paullo longiore, apice nonnihil dilatato; $3-8$ brevissimis compressis, sub-perfoliatis, sensim latioribus; clava magna ovata.

Rostrum capite sesqui longius, crassum, perparum arcuatum, supra 5-carinatum, carinula media apice bifida; mandibulae validae apice bidentatae. 
Oculi rotundati laterales valde remoti, haud prominuli.

Thorax suborbiculatus, convexus, basi apiceque truncatus.

Elytra oblongo-ovata inflata.

Pedes robusti; tibiae anticae retrorsum arcuatae, apice appendice spathuliformi magno terminatae; posticae crassae hispidae, apice valde dilatato cyathiformi; tarsi breviusculi unguiculis tenuissimis.

Genus valde paradoxum, prope Strpulum et Trachonem operis Cel. Schönherr forte ponendum.

139. EuPHYA STES FUCICOLA: oblongus, nigro-piceus nitidus, glaber; thorace parce punctato; elytris profunde striato-sulcatis; capite, thoracis elytrorumque limbo omni late pedibusque testaceo-pallidis, genubus, tibiarum apice tarsisque infuscatis.

I $a r$. b. corpore subtus etiam testaceo-pallido.

Var. c. tota pallide testacea, oculis nigris, thorace elytrisque in medio nonnilhil, genubus, tibiarum apice tarsisque magis infuscatis.

Longit. cum rostro $3 \frac{1}{4}-3 \frac{1}{3}$ lin. Latit. $1 \frac{1}{2}-1 \frac{2}{3}$ lin.

In arena littorali insulae Edgecombe sub fucis e mare rejectis copiose legit D. Frankenhaeuser.

140. Tracho des ror r I Us: elongatus, nigro-fuscus, brunneo-squamulosus; thorace elongato, ruguloso, medio subcarinato, antrorsum transverse quadrituberculato; elytris lacunosis, trifariam tuberculatis, tuberculis magnis alte intumidis.

Longit. sine rostro 2 lin. Latit. $\frac{3}{4}$ lin. 
Habitat in insula Sitkha rarissime; sub ligno caeso a D. Holmberg repertus.

141. Trachodes Quadruterculatus Molschulsky: brevis, ferrugineus, griseo - squamulosus; rostro, antennis tarsisque rufo-testaceis; thorace oblongo, lateribus rotundato, dorso cinereo-trilineato; elytris subovatis convexis, profunde crenato - striatis, testaceofasciculatis, disco tuberculis quatuor in quadratum dispositis. (Mas.)

Femina: major, thorace etiam tuberculis quatuor brunneo-holosericeis in medio transversim positis notato.

Longit. sine rostro $1-1 \frac{1}{2}$ lin. Latit. $\frac{1}{2}-\frac{3}{4}$ lin.

Stueneus ecidnitunencrutes, Motschulsky. Bullet. de la Soe. de Moscou. 1845. IV. p. 375. 64. Tab. VII. fig. 4.

Habitat in insula Sitkha in truncis et supra corticem pinorum frequens; DD. Pippingsköld, Franlicnhaeuser et Holmberg. Specimen ibique captum ad describendum etiam communicavit D. Motschulsliy.

142. Ceutor uy cur pusio: nigro-piceus, parce griseosquamosus, thoracis apice, elytrorum parte posteriore pedilousque rufo-piceis; capite thoraceque profunde punctatis; fronte foveola magna minus profunde impressa; thorace antice constricto, in medio utrinque acute dentato, lateribus densius griseo-squamoso; elytris profunde sulcatis, striis punctatis, interstitiis rugulosis, squamulis griseis subundulatim variegatis; femoribus muticis.

Longit. sine rostro 1 lin. Latit. $\frac{7}{2}$ lin.

Habitat in insulae Sitkhae horto in arbustis frequens; D. Frankenhaeuser. 
Ad Stirpis 1. Manipulum 1. operis Schönherr pertinet.

143. HyLURGS NigRINUS: elongatus, ater, opacus, antennarum capitulo tarsisque obscure rufis; thorace oblongo, antrorsum nonnihil angustato, profunde punctato, subcarinato; elytris profunde punctato-striatis, interstitiis valde rugosis.

Longit. $2 \frac{2}{5}$ lin. Latit. 1 lin.

In insula Sitkha unicum tantum specimen in volatu cepit D. Frankenhaeuser.

Statura omnino II. atri. Fabr. sed multo major, thorace fortius punctato et elytris magis rugoso-scabrosis diversus.

144. HyLURG US SER I CEUS Eschscholtz: Mannerh. Bullet. de la Soc. de Moscou. 1843. p. 296. 256.

Var. b. minor, niger, atro-hispidus, pedibus piceis, elytris obsolete cinereo-squamulosis.

Habitat in insulae Sitkhae truncis pini sub cortice rarius;

D. Frankenhaeuser.

145. HyLuRGUS OBESUS Eschscholtz: Mannerh. Bullet. de la Soc. de Moscou. 1843. p. 296. 257.

Var. b. thorace obsoletius carinato, elytris rufobrunneis.

Var. c. pallide testacea, capite obscuriore, thorace vix carinato.

Habitat in insula Sitkha, sub cortice truncolorum pini non infrequens; DD. Frankenhaeuser, Pippingsköld et Holmberg.

146. Hycurgus pumlus: Bannerh. Bullet. de la Soc. de Moscou. 1843. p. 297. 259. 
Var. b. capite thoraceque nigro-piceis; cetera ut in $a$.

Var. c. nigro-picea tota, pedibus tantum rufopiceis.

Habitat in insulae Sitkhae truncis pini sub cortice frequens;

DD. Frankenhaeuser et Pippingsköld.

147. Bostrichus InteRRUPtus: oblongus, cylindricus, nigro-piceus, nitidus, longe et parce griseo-pilosus; thorace subquadrato, angulis rotundatis, profunde punctato, antrorsum valde rugoso; elytris profunde striato-punctatis, punctis versus apicem plus minusve evanescentibus, apice circulatim retusis, ambitu quadridentato, dente tertio longiore incurvo.

Var. b. elytris obscure rufo-castaneis; cetera ut in $a$.

Longit. $2-2 \frac{1}{4}$ lin. Latit. $\frac{3}{4}-1$ lin.

Habitat in insulae Silkha, truncis pini sat frequens; DD. Frankienhaeuser et Holmberg.

148. Bostricuus tridexs Ménétriés: oblongus, cylindricus, rufo-castaneus, subnitidus, longe et parce griseopilosus; thorace antrorsum infuscato, quadrato, angulis rotundatis, postice sublaevi nitido, lateribus et antice valde rugoso; elytris ad apicem usque profunde striato-punctatis, apice circulatim retusis, ambitu tridentato, dente intermedio majore.

$V a r . b$. tota rufo-ferruginea, oculis tantum nigris; elytris apice praeter dentes tuberculo minutissimo supra in ambitu circulari notatis.

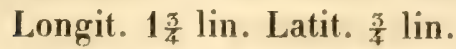


Sub cortice truncorum pini, in insula Sitkha rarius; Dl). Pippingsköld et Holmberg.

Praccedente brevior, thorace breviore, basi laevigato, elytrorum striis inter se magis remotis usque ad apicem continuatis punclisque striarum minus approximatis, ut et apice tridentato distinctus.

1.19. Bostricuus coxcinves: oblongus, cylindricus, nigro-piceus, subnitidus, densius longe flavescenti-piIosus; thorace oblongo-quadrato, basi truncato, antrorsum rotundato, supra creberrime punctato, anterius valde rugoso, postice in medio carinato; elytris concinne punctulatis, punctis subseriatis, apice circulatim retusis, ambitu tridentato, dente medio interdum appendiculo dilatato truncato, tertio longiore apice subclavato; antennarum clava tarsisque ferrugineis.

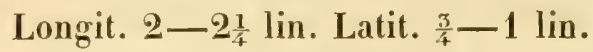

Habitat sub cortice truncorum pini, in insula Sitkha non infrequens; D. Frankenleaeuser

150. Bostrichus SEMCASTANEUS: oblongus, nigro-piceus, nitidus, parce et longe griseo-pilosus; thorace oblongo - rotundato, basi elytris angustiore, crebre et profunde punctato, obsolete carinato; elytris integris castaneis, profunde striato-punctatis, interstitiis seriato-punctatis, pilis versus apicem in lineas dispositis; antennis pedibusque rufis.

Var. b. elytris etiam nigro-piceis.

Var. c. tota rufo-testacea oculis tantum nigris; forsan immatura.

Longit. $2 \frac{1}{4}-2 \frac{1}{2}$ lin. Latit. $\frac{5}{6}-1 \frac{1}{4} \operatorname{lin}$.

Habitat in insulae Sitkhae truncis pini sub cortice frequens;

DD. Frankenhaeuser et Pippingsköld. 
B. Septentrionis Leconte (Mannerh. Bullet. de la Sor. de Moscou 1843. p. 298. 261.) aftinis, sed major, latior, thorace antrorsum minus angustato puncturaque profundiore et crebriore diversus.

151. BostricuUS AFFA ER: oblongus, obscure brunneus, opacus, longe griseo-villosus; thorace oblongo, antrorsum angustato, basi elytrorum latitudine, crebre punctato, antice rugoso, dorso obsolete carinato: elytris cylindricis, apice subtruncatis declivibus, dense et profunde striato-punctatis, antennis pedibusque ferrugineis.

Longit. $1 \frac{1}{2}$ lin. Latit. $\frac{1}{2}$ lin.

In trunco pini insulae Sitkhae legit D. Pippingstiöld.

152. Bostricuus ritiduzus: Mammerh. Bullet. de la Soc. de Moscou 1843. p. 298. 263.

Var. b. plus minusve rufescenti-picea.

Var. c. pallide testacea, thorace antrorsum capiteque infuscatis; certe individua nuper exclusa.

Sub cortice truncorum pini insulae Sitkhae passim copiose occurrit; DD. Frankenhaeuser, Pippingsköld et Holmbery.

153. Bostricirus cavif rons: Mannerh. Bullet. de la Soc. de Moscou 1843. p. 297. 260.

$V a r . b$, thorace infuscato, relicta tantum area postica transversa pallida plus minusve dilatata, antrorsum biloba; cetera ut in $a$.

Var.c. thorace ut in $a$ et $b$, sed elytris totis pallidis, versus apicem plus minusve infuscatis.

Habitat in truncis pini sub cortice, in insula Sitkha sat frequens; DD. Frankenhaeuser, Pippingsköld et Holmbery. 
154. Cis TRIDENTATUs: oblongus, rufo-testaceus, pube brevi cinerea rigida adspersus, in elytrorum apice praesertim condensata; fronte acute bidentata, dentibus basi dilatatis supra concavis; thorace convexo, crebre punctato, antrorsum angustato, in dentem elevatum apice subemarginatum producto; elytris creberrime punctulatis, punctis leviter impressis. (Mas.)

Femina: capitis margine antico incrassato, emarginato, thorace apice rotundato inermi.

Longit. $\frac{2}{5}-\frac{3}{4}$ lin. Latit. $\frac{1}{2}-\frac{2}{3} \operatorname{lin}$.

Irabitat in boletis arboreis insulae Sitkhae sat frequens;

D. Frankenhaeuser.

155. Cis i I R M TUS: oblongus, rufo-testaceus, pube brevissima flavescenti rigida densius adspersus; fronte profunde excavata, dentibus duobus brevibus concavis armata; thorace lateribus rotundato, antice rotundatoproducto inermi, subtiliter crebre punctulato; elytris crebre leviter punctulatis, rugulosis, punctis majoribus remotis intermixtis, antrorsum obsolete substriatis. (Mas.)

Femina: fronte excavata, margine apicali incrassato, emarginato.

Longit. $\frac{3}{4}-1$ lin. Latit. $\frac{1}{2}-\frac{2}{3}$ lin.

Cum praecedente copiosius legit D. Franḳenhaeuser.

156. Cis Americanus Motschulsky: oblongus, rufo-testaceus, pube brevissima cinerea rigida densius adspersus; fronte excavata, margine antico profunde inciso; thorace postice truncato, lateribus modice rotundato ibique late explanato, subtiliter crebre et concinne punctato; elytris concinne sat crebre punctulatis. (Mas.) 
Femina: fronte deplanata, margine antico profunde inciso.

$V a r . b$. tota ferrugineo-testacea, capite infuscato.

Longit. $\frac{3}{4}$ lin. Latit. $\frac{1}{3}$ lin.

Habitat in insulae Sitkha boletis arboreis minus frequens;

DD. Pippingsköld et Frankenhaeuser.

157. Corticaria trisignata: elongata, rufo-ferruginea, glabra, subnitida; thorace profunde et rude punctato, lateribus utrinque acute quadridentato ibique et postice marginato, in disco longitudinaliter bifoveolato; elytris pallidius testaceis, profunde punctato-striatis, mox ante medium utrinque macula magna postice tridentata margini contigua et pone illas sutura postice nigro-infuscatis.

Longit. $1 \frac{1}{4}-1 \frac{1}{2}$ lin. Latit. $\frac{1}{2}-\frac{2}{5} \operatorname{lin}$.

In fungo arboreo insulae Sitkhae semel a D. Frankenhaeuser copiose lectus.

Species elegantula, inter hujus generis maximas, forma peculiari insignis.

158. Corticaria SPINUlosa: elongata, nigro-picea, subnitida, tenue pubescens; antennarum basi pedibusque rufo-testaceis; capite thorace angustiore; thorace subrotundato, convexiusculo, sparsim profunde punctato, lateribus denticulis acutis armato, postice distincte foveolato; elytris profunde striato-punctatis, interstitiis subrugulosis.

Longit. $\frac{2}{3}$ lin. Latit. $\frac{1}{3}$ lin.

Habitat in insula Sitkha rarius; in domo a D. Holmberg inventa. 
C. Longiconn Herbst statura similis, sed multa minor, capite angustiore, thorace lateribus acute denticulatis, postice profunde foveolato elytrisque aliter sculpturatis diversa.

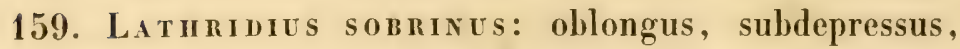
glaber, obscure rufo-testaceus, oculis nigris, antennis pedibus elytrisque ferrugineo-testaceis; thorace oblongo-quadrato, angulis anticis rotundato-productis, medio longitudinaliter bifoveolato, intra angulos anticos et posticos excavato - foveolato; elytris latioribus ovalibus, profunde striato-punctatis.

Longit. 1 lin. Latit. $\frac{2}{3}$ lin.

In insula Sitkha volitantem cepit D. Holmberg

L. Pnotexsicoll Mannerh. brevior, thoracis structura et elytris latioribus praeterea ab illo diversus.

160. RiIzopHagus DIMDItus Eschscholtz: Mannerh. Bullet. de la Soc. de Moscou 1843. p. 300. 268. (")

Var. c. ut a, sed elytris totis obscure rufis, interdum in medio infuscatis.

Var. $d$. etiam ut in $a$, sed elytris basi angustius rufescentibus, rufedine extrorsum dilatata.

Sub cortice truncorum pini, in insula Sitkha frequens: DD. Frankenhaeuser et Pippingsköld.

161. Rinzopha gus s calpturatus : linearis, subdepressus, nigro-piceus, nitidus; thorace oblongo-quadrato, concinne minus remote punctato; elytris punctatostriatis, apice subtruncatis; antennis pedibusque rufis.

(") Durch einen Druckfehler sind in der Diagnose die Worte punctatostriatis nach elytris weggelassen, welches ich also hier berichtige. 
Longit. $1 \frac{1}{3}$ lin. Latit. $\frac{1}{2}$ lin.

Excipulo in insula Sitkha a D. Frankenhaeuser semel captus.

Praecedente minor, brevior, thorace breviore, densius punctato, elytris versus apicem haud attenuatis, apice subtruncatis punctisque striarum magis approximatis distinctus.

162. Silvarus dextatus Fabricius: elongatus, nigropiceus, opacus, tenue pubescens; thorace oblongoquadrato, utrinque sexdentato, dentibus obtusis, supra punctulato, punctis versus latera condensatis, postice foveis duabus majoribus divergentibus impresso; elytris subtilissime elevato-lineatis, interstitiis dense striato-punctatis; pedibus rufo-piceis.

Longit. $1 \frac{3}{4}$ lin. Latit. $\frac{1}{2}$ lin.

Lycrus nentatus, Fabr. Ent. Syst. I. 2. p. 503. 4. Syst. El. II. p. 561. 5 .

Silvanus denticollis, Dejean Cat. 3-ème édit. p. 338.

In insula Sitkha, in saccharo crudo mercatoribus allato semel legit D. Frankenhaeuser.

163. Pedicus subcarinatus: elongatus, deplanatus, fusco-castaneus; capite antice utrinque impresso; autennarum articulis 4,6 et 8 minoribus; thorace subquadrato, creberrime punctato, medio subcarinato, ad carinulam utrinque longitudinaliter impresso, lateribus subrectis reflexis, quadridentatis, dentibus 1 et 2 obtusis, 3 et 4 acutiusculis, spatio interjacente sinuato; elytris subtilissime punctulatis, stria suturali profunde impressa; antennis pedibusque rufescentibus.

Longit. 2 lin. Latit. $\frac{2}{5}$ lin. 


\section{2}

Habitat in continenti Americes borealis ad fontes Kaknu; D. Frankenhaeuser.

P. fusco Erichs. (Cucujus dermestoides Gyllenh. Biophloeus ID. Dej.) affinis, sed longior, praesertim elytris, practerea thoracis structura ab illo bene distinctus.

164. Brontes truncatus Motschulsky: oblongus, deplanatus, brunneo-castaneus opacus, rugoso-punctatus, thorace laterilus denticulato, angulo antico producto, dorso foveis quatuor oblongis; elytris brevioribus profunde punctatis, intra marginem argute costatis, apice subtruncatis ibique margine late explanato.

Longit. $2 \frac{1}{5}$ lin. Latit. 1 lin.

Motschulsky, Bull. de la Soc. de Moscou 1845. I. p. 92. 267.

Habitat in insula Silliha et in California boreali, teste D. Motschulskiy, e cujus museo specimen ad describendum benevole mihi transmissum fuit. Etiam in California boreali a D. Cygnaeo captus.

Br. Flavipedr Fabr. affinis, sed elytris multo brevioribus, profundius punctatis, argutius ad latera costatis, apice subtruncatis, explanato-marginatis distinguendus; a Br. dubio Fabr. autem, cui statura el sculptura magis propinquus, differt thorace lateribus subtilius denticulato, angulisque anticis multo minus acute productis.

165. Prionus Califoricus Motschulshy: convexus, obscure castancus, nitidus; fronte canaliculata; thorace brevissimo, utrinque lateribus acute tridentato; elytris confertim punctulatis, obsolete tri-costatis; antennis longitudine dimidii corporis, articulo tertio elongato, sequentibus vero infundibuliformibus sensim minoribus, ultimo oblongo apice oblique rotundato. (Mas.)

Longit. 16 lin. Latit. $6 \frac{1}{8}$ lin. 


\section{3}

Femina: differt elytris sesqui longioribus, antennis pedibusque tenuioribus.

Longit. 24 lin. Latit. 8 lin.

Motschulsky, Bullet. de la Soc. de Moscou 184ว. p. 89. 260. Tab. II. fig. 9.

Habitat in California boreali; D. Cygnaeus, qui mihi marem dedit: etiam in insula Sitkha sec. D. Motschulskiy, e cujus museo feminam descripsi.

Pr. poculari Schönh. ex America boreali valde affinis, sell differt thorace dorso magis aequali convexiore, lateribus. que acutius dentato, elytris subtilius punctatis et antennis brevioribus, articulis magis contractis.

166. Rosalia funebris: lineari-elongata, depressa, nigra; froute, scutello, corpore subtus thoraceque pube caerulescenti - cinerea dense obtectis; hoc transversim quadrispinoso, macula subrotundata media atro-sericea; elytris atro-sericeis, utrinque fasciis duabus latis extrorsum attenuatis, puncto minuto his interposito, apiceque singulatim rotundato, cinereis; his coloribus e pube brevi condensata effectis; antennis pedibusque nigris cinereo-annulatis.

Longit. 13 lin. Latit. 4 lin.

Rosama funebra Motschulsiin, Bullet. de la Soc. de Moscou 1845. I. p. 87. 256. Tab. II. fig: 8.

Habitat in insula Sitkha, teste D. Motschulskiy, qui typum descriptionis a se factae benevole mihi transmisit.

\section{P LEC T R U R A Dejean.}

Antennae 11-articulatae, setaceae vel apicem versus sensim tenuiores; articulo primo incrassato, oblongo - ovato; se- 
cundo brevissimo globoso; ceteris cylindricis; tertio angustato, elongato, primo sesqui longiore; 4-to antecedente dimidio breviore; $5-10$ subaequalibus; ultimo acuminato.

Palpi articulo ultimo oblongo-ovato, subacuminato.

Thorax latitudine vix longior, inaequalis, lateribus in medio utrinque spina valida armatus, adjectis spinulis aliquot minutis.

Scutellum minutum, subchadratum.

Elytra basi thoracis basi fere duplo latiora, humeris rotundatis subserratis, in medio parum dilatata, latitudine fere duplo longiora, valde convexa, margine versus apicem crenulata, apice ipso acumine singulatim producta.

Pedes validi, tibiis intermediis extrorsum in medio dente obtuso armatis.

Genus habitu Dorcabrosi propinquum.

167. Prectrura spinicauda Eschscholtz: obscure brunneo-picea, parce griseo-pubescens, supra maculis e pilis griseo-flavescentibus adspersa, undique profunde rugoso - punctata; elytris seriebus utrinque quinque e tuberculis glabris nitidissimis, quarum prima et quarta versus medium tantum continuatae.

Mas differt statura paullo breviore, antennis pedibusque nonnihil validioribus.

Var. $b$. tota dilutius rufo-picea; cetera ut in $a$.

Longit. $4 \frac{2}{3}-5 \frac{1}{3}$ lin. Latit. 2 lin.

Dejean, Cat. 3-ème édit. p. 373. 


\section{5}

Habitat in insula Sitkha, inter ligna caesa in pinetis rarius; DD. Frankenhaeuser, Pippingskïld et IIolmbery.

168. RHA iun investigator: nigrum, cinereo-pubescens; thorace deplanato, lateribus utringue dente aucto armato, linea longiludinali media glabra nitidissima; elytris valde rugosis, pone medium haud angustatis, apice rotundatis, dorso utrinque tricarinatis, maculis numerosis irregularibus testaceis, his in fascias transversas, una ante, altera pone medium obsolete confluentibus, prima antice, secunda postice nigredine confuse limitatis.

Longit. $6 \frac{1}{5}-7 \frac{1}{3}$ lin. Latit. $2 \frac{1}{4}-2 \frac{9}{3}$ lin.

In insula Sitkha, e larvis in ligno pini repertis a DD. Frankenhaeuser et Holmberg educatus.

RH. Linesto Oliv. valde affine, elytris autem posterius haud attenuatis, supra magis rugosis, costis illorum minus ele vatis anterius obsoletioribus fasciisque transversis nigris minus conspicuis species reapse liversa mihi videtur.

169. Paсnута liturat Kirby: atra, villoso-incaua; thorace lateribus in medio dente obtuso armato; elytris versus apicem singulatim attenuatis dehiscentibus, apice ipso truncato, punctulatis, livido-testaceis, glabris, sutura margineque anguste et singulo lituris tribus obliquis nigro-brunneis, anticis duabus subparallelis interdum confluentibus.

Longit. $6-9$ lin. Latit. $2 \frac{1}{2}-3 \frac{1}{2}$ lin.

Richardson, Fauna Bor. Amer. IV. p. 178. 238.

Habitat in continenti Americes borealis ad sinum Kenai; DD. Etholén, Frankenhaeuser et Holmberg

P. Lasen Linné proxima, sed spinis lateralibus thoracis 


\section{8}

oblusis haud recurvis et magis in medio positis, punctura multo subtiliore, elytris posterius magis attenuatis horumque signaturis aliis abunde distincta.

170. Donacia Germari Eschscholtz: Mannerh. Bullet. de la Soc. de Moscou. 1843. p. 306. 281. 1846. p. 515. Lacordaire Monogr. des phytophages. I. p. 191. 54.

Var. d. thorace obscure viridi-aeneo, elytris obscure cupreis, pedibus totis rufo-ferrugineis.

In insula Sitlha, in gramine ad ripas lacuum sat frequenter legit D. Frankenhaeuser. IIanc speciem in copula cum D. FLIVIPENi Eschsch., quem marem esse autumat, a se repertam fuisse scripsit D. Holmberg; an accidens tantum ad nuptias insectorum in Germar Mag. IV. p. 408. jam commemoratas attribuendum. Adsunt enim mares et feminae utriusque speciei notis ut in congeneribus inter se distincti.

171. G A L E R U C A U C T U os A: oblonga, subdepressa, nigra, tenuissime griseo - pubescens; capite creberrime punctulato, inter antennas canaliculato; thorace transverso, lateribus in medio angulato-dilatato, basi ante scutellum profunde inciso, supra remote profunde punctato, in medio tenue canaliculato foveaque magna utrinque profunde impresso; elytris elongatis, pone medium nonnihil dilatatis, creberrime praesertim posterius punctatis, margine laterali pallide testacea.

Longit. $2 \frac{1}{2}$ lin. Latit. $1 \frac{1}{4}$ lin.

Habitat in continenti Americes borealis ad sinum Kenai; D. Etholén.

A Gall. Sigittania Gyllenh. quae sec. Kirby (Richardson Faun. Bor. Amer. IV. p. 219.) etiam in America boreali occurit, thoracis incisura postica profundiore, elytrorum punctura creberrima ut et colore species omnino diversa. 
172. Gonioctena AFFinis Schöhher: oblongo-ovata, convexa, nigra; thorace transverso, subplano, lateribus ultra medium parallelis, dein apicem versus sub)oblique angustato, angulis anticis acutis, dorso remote punctato, punctis profundis, versus latera crebrioribus et ad basin intra angulos confluentibus, margine laterali, antrorsum latius, lineolaque media apicis rufo-ferrugineis; elytris tenue punctato-striatis, punctis striarum pone callum humeralem et versus apicem hinc inde irregularibus, rufo-ferrugineis, singulo maculis quinque bifariam positis nigris, quarum duate interiores magnae oblongae, exteriores prima ef tertia minores intermedia vero majore subtransversa; antennis dimidia parte, margine segmenti ultimi abdominalis tibiisque extrorsum rufo-testaceis.

Longit. $2 \frac{1}{2}$ lin. Latit. $1 \frac{1}{2}$ lin.

Dejean, Cat. 3-ème édit. p. 427.

Chrysomela affixis Gyllenhal, Ins. Svec. III. p. 483. 28. Var. c. - Suffrian in Linnaea Entomologica V. p. 218. 153.

Habitat in continenti Americes borealis ad fontes Kaknu; D. Frankenhaeuser.

173. Litochrus Brunnipenris: ovatus, convexus, rufotestaceus, nitidus; capite subtilissime punctulato; thorace transverso laevi, angulis rotundatis; elytris brunneo-castaneis, subtiliter punctulatis, apice oblique truncatis, abdomine brevioribus; antennarum clava nigro - fusca.

Longit. $\frac{3}{4}$ lin. Latit. $\frac{2}{3}$ lin.

Habitat in insula Sitkha, in fungis; D. IIolmberg.

174. Agathidiumaxgulare: subglobosum, nigrum, ni- 


\section{8}

tidum; thorace subtiliter punctulato, lateribus anguste angulisque posticis late rufo-ferrugineis pellucidis; elytris profunde et sat crebre punctatis, stria suturali praesertim posterius valde impressa.

Longit. $1 \frac{1}{3}$ lin. Latit. 1 lin.

Habitat in insula Sitkha rarius; sub cortice truncorum pini a D. Pippingsköld inventum; in volatu a D. Holmberg captum.

175. Agathidem concinem: rotundato-ovatum, piceotestaceum; thorace transverso, vix punctulato, sublaevi, lateribus pallide diaphano; elytris crebre et profunde punctatis, stria suturali posterius impresssa.

Var. b. pallide testacea, oculis antennarumque clava tantum obscure piceis.

Longit. $\frac{3}{4}-1$ lin. Latit. $\frac{2}{5}-\frac{3}{4}$ lin.

Agathidiom castaneum Ménétriés in litteris.

Excipulo e gramine in insula Sitkha a D. Frankenhaeuser saepius lectum; sub cortice pini a D. Pippingsköld repertum.

Ob jan exstans Anisotoma (Lerodes) castanea Herbst, Illig. Sturm nomen mutandum esse putavi.

176. Agathidum rotundud: hemisphaericum, globosum, nigrum, laevissimum, nitidum; thorace magno transverso, lateribus valde rotundato, margine late rufo-pellucido; elytris stria suturali nulla; antennarum basi pedibusque rufo-piceis.

Longit. $\frac{2}{3}$ lin. Latit. $\frac{1}{2}$ lin.

In insula Silkha iisdem locis cum praecedente a D. Franlienhucuser copiose, sub lapide a D. Pippingsköld semel captum. 
177. Coccinella transversoguttata Say.: oblongohemisphaerica, nigra; capite maculis duabus thoraceque angulis anticis albidis; elytris fulvis, fascia communi pone basin latera non attingente et singulo macula disci alteraque ante apicem, transversis nigris.

Longit. $3 \frac{1}{3}$ lin. Latit. $2 \frac{1}{2}$ lin.

Faldermann. Coleopter. illustr. (Mlém. de l'Icad. de St.Pétersb.) II. p. 45̆. 97.

Mulsan, Spec. des Col. Trim. Sécurip. p. 117 26. Var. $\Lambda$. Coccinelea 5-мotata Kirby in Richardson Faun. Bor. Amer. IV. p. 230. 313.

Ifabitat in continenti Americes borealis, ad fontes Kalinu:

D. Frankenhaeuser: ad sinum Kenai; D. Holmberg.

178. Bryats Albionca Motschulsliy: nigra, nitida, antennis fuscis, harum articulis j-to et 6-to dilatatis, palpis, elytris pedibusque dilute castaneo-rufis; thorace postice trifoveolato, fovea intermedia antescutellari minore; tibiis posticis inflexis, apicem rersus dilatatis.

Longit. $\frac{3}{4}$ lin. Latit. $\frac{1}{4}-\frac{1}{3}$ lin.

Motschulstiy, Bullet. de la Soc. de Mosenu 18'.5. I. p. 43. 111. Tab. III. fig. 2. IV. p. 360. 37. Tab. VI fig. 3.

In insula Sitkha a DD. Frankenhaeuser et Holmberg excipulo capta; occurrit etiam in California boreali sec. T). Motschulsky.

Variat secundum descriptionem a v. el. Motschulsky l. r. datam colore antennarum, elytrorum pedumque dilutiore. ("Mäklin ")

179. Trimu u chavicorie Mäklin: elongatum, nigrum, nitidum, antennis pedibusque testaceis, illis breviusculis, articulo ultimo fortiter incrassato; capite late 


\section{0}

biimpresso; thorace brevi postice foveolis tribus sulco transversali semicirculari connexis impresso; elytris thorace dimidio longioribus, subtiliter parce punctulatis.

Longit. $\frac{2}{3}$ lin. Latit. $\frac{1}{6}$ lin.

In insula Sitkha a D. Holmberg detectum.

Euplecto brcolon paulo longius sed vix latius. Ob antennarum structuram generi Trino adnumeratum. ("Mäklin")

180. Euplectus parviceps Mäklin: elongatus, rufotestaceus, antennis pedibusque dilutius testaceis; capite parvo, antice longitudinaliter profunde impresso, punctis duobus sat magnis in vertice transversim positis; thorace brevi, basin versus angustato, in disco bipunclato, ante basin profunde transversim impresso et ad angulos basales utrinque foveolato; elytris thorace duplo longioribus.

Longit. parum ultra 1 lin. Latit. $\frac{1}{4}$ lin.

Excipulo locis graminosis in insula Sitkha a DD. Frankenhaeuser et Holmberg rarissime inventus. ("Mäklin") 


\section{VERZEICHNISS}

\section{aller aus den nord-amerikanischen Ländern des russi- schen Reiches in entomologischen Schriften bisher er- wähnten Käfer, nach dem natürlichen Systeme geordnet.}

Damit dieses Verzeichniss zugleich als Register dienen möchte, hahe ich bei jeder Art angegeben, wo die Beschreibung oder sonst eine gedruckte Erwähnung derselben aufzufinden ist. Der Kiirze wegen bedeuten dabei:

A. mein Beitrag zur Käfer-Fauna der Aleutischen Inseln, der Insel Sitkha und Neu-Kaliforniens.

B. mein Nachtrag zur Kiifer-Fauna der Aleutischen Inseln und der Insel Sitkha.

C. mein zueiter Nachtrag zur Käifer-Fauna der Nord-Amerilianischen. Länder des Russischen Reiches. (Die Nummern beziehen sich auf die Species derselben in den verschiedenen Abhandlungen.)

Cat. die Käfer Russlands von Motschulsky.

Bull. Bulletin de la Société Impériale des Naturalistes de MIoscou.

\section{CARABICA.}

Notrophilus Dumeril.

sylvaticus Eschsch. (A. 22.)

Sitkha - sub lapidibus et

ligno caeso non infrequens.

Pelophila Dejean.

Eschscholtzii Sturm. (A. 20.) Unalaschka.

\section{Nebnia Latreille.}

metallica Eschsch. (A. 15.) Linalaschka; Sitkha.

Gebleri Eschsch. (A. 16.) (") Sitkha.

(*) Obgleich Herr v. Motschulsky, nach Ansicht der friheren Sammlung des Grafen Dejean be- 
"macrobephala Motsch. (Cat.p. 72.) Umlaschka.

gregaria Eschsch. (A. 17.) Unalasehka.

Mannerheimii Eschsch. (A. 18.

Sitkha; Edgecombe.

Sahlbergii Eschsch. $\left(^{\star}\right)$ (A. 19.) Sitkha. - sub truncis arborum emortuar. rarius.

carbonaria Eschsch. (C. 4.) Ins.

St. Pauli. Siquria Mann.

Leistus Fröhlich.

ferruginosus Mannerh. (A. 14. C. 3.) Sitkha.

(ferrugineus Eschsch.

\section{Cychros Fabr.}

angusticollis Eschsch.

(A. 8 .

Unalaschka; Sitkha.

hauptet (Bullet. 183̈1. I. p. 363.), dass diese nur eine Varietät der metallica sei, so kann ich ihm nicht beistimmen, indem das Exemplar meiner Sammlung alle von Eschscholtz und Dejean so genau beschriebene specifische Charaktere darbietet.

(*) Die Ansicht des Herrn v. Motschulsky's, dass meine Var.b. der $N$. Sahlbergii eine von dieser verschiedene Art wäre, die er als N. violacea sibi (Cat. p. 73.) zıemlich entfernt von jener aufstellt, kann ich nicht theilen; es ist in der Abart ausser der Farbe eines neulich entwickelten Individuums, gewiss durchaus gar nichts vom eigentlichen Typus specifisch Eigenthümliches. marginatus Eschsch. (A.9.) Unalaschka; Sitkha.

* reticulatus Motsch. (C. 1.) Unalaschka?

\section{Carabus Linné.}

Vietinghovii Adams. (C. 2.) Litt. Bor, Amer. Fret. Behringii.

Chamissonis Eschsch. (A. 11.) Unalaschka.

baccivorus Eschsch. (A. 12.) Unalaschka.

\section{Loricera Latreille.}

decempunctata Eschsch. (A 26.) Sitkha. - sub lapidibus minus frequens.

Drschirius Bonelli.

* transmarinus Ménétr. (Cat. p. 18.) Sitkha.

\section{Harpalus Latreille.}

somnulentus Eschsch. (A. 77.) Sitkha. - sub lapidibus haud vulgaris.

Stenolophus Ziegler.

nigrinus Eschsch. (A. 80. C. 14.) Sitkha. - sub lapidibus et ligno caeso sat frequens.

*quadripunctatus Ménétr. (Cat. p. 22.) Unalaschka.

*axillaris Ménétr. (Cat. p. 22.) Sitkha.

HypinerPes Eschsch.

herculaneus Mannerh. (A. 52.) Sitkha. 
validus Eschsch. (A, 533.) Sit- riparia Eschsch. (A. 64.) Sitkha. kha. - sub lapidibus et ligno similis Ménétr. (C. 9.) Ins. St. caeso frequentissime. Georg.; Ins. Stephens.

ameth stinus Eschsch. (А. 5̌4.) *quadricollis Ménétr. (Cat.p.5̌.) Sitkna. - iisdem locis non infrequens.

castaneus Eschsch. (A. 55.) Sitkha. - in truncis putridis et sub lapidibus sat frequens. brunneus Eschsch. (A. 56 . Sitkha.

\section{Botimopteres Chaudoir.}

orinomum Leach. (C. 7.: Cont. Amer. bor.

adstrictus Eschsch. ( . 59.) Unalaschka; Sitkha. - sub lapidibus et truncis arb. deciduis frequens

* commixtus Chaud. (Bull. 1850. III. p. 135.) Sitkha.

seriepunctatus Mannerh. (A. 60.) Sitkha.

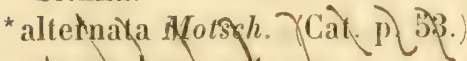
Amen. bor. ardt

${ }^{*}$ fuscoaeneus Eschsch. (A. 58.) Sitkha.

\section{Cryobius Chaudoir.}

${ }^{*}$ rugulosus Eschsch. (C. 8.) Unalaschka.

ventricosus Eschsch. (A. 61.)

Unalaschka.

pinguedineus Eschsch. (A. 62.)

Unalaschka.

*hyperboreus Ménétr. (Cat.p.

54.) Ins. St. Georgii.

empetricola Eschsch.

Unalaschka; Atkha.
Ins. St. Georgii.

Miscodera Eschscholtz.

insignis Mannerh. (C.10.) Sitkha.

Leinos Megerle.

melanogastricus Eschsch.(A. 72.

C. 12.) Unalaschka.

Eschscholtzii Chaud. (C. 13.)

Amer. bor. occ.'Ins. St. Pauli.

Bradytus Stephens.

*glacialis Mínétr. (Cat. p. 62.) Litt. mar. glac. Amer. bor. occid.

\section{Amara Bonelli.}

littoralis Eschsch. (A. 66.) Sitkha. - sub lapidibus et ligno caeso sat frequens.

\section{Cena Zimmermann.}

interstitialis Eschsch. (C. 11.) Contin. Amer. bor. occ. remotestriata Eschsch.(A. 69.) Unalaschka. *indistincta Motsch. (Cat. p. 59.) Unalaschka. * compacta Motsch.f(Cat. p. 59.) Sitkint.

\section{Calathus Bonelli.}

(A. 63.) ingratus Eschsch. (A. 35.) Unalaschka. 


\section{4}

Anchomenus Bonelli.

mollis Eschsch. (А. 43.) Una-

laschka; Sitkha; Mtkha.

Var. a. brunnescens Sahlb.

(C. ๖.) Atkha.

Var. a. breviusculus Sahlb.

(C. 5.) Atkha.

* plankennis Motsch. (Catk, p. (68.) Sitkhà.

* Mdschulskii Maikzerh.

Anchemenoides (Agonothof rax) Motsch. (Cat. p. 68.)

Unalaskhka.

strigicollis Mannerh. (C.6.) Con-

tin. Amer. bor. occ.

*octocolus Chaud. (Cat. p. 69.)

Amer. oce. arct.

\section{Patrobus Megerle.}

foveocollis Eschsch. (A.32.) Unalaschka.

fossifrons Eschsch. (A. 33.) Unalaschka.

aterrimus Eschsch. (A. 3\%.) Sitkha.

Trechus Clairville.

spectabilis Mannerh. (C. 15.) Sitkha.

chalybeus Sturm. (A. 83.) Unalaschka.

oblongulus Mannerh. (C. 16.) Sitkha.

ovipennis Motsch. (C.17.) Sitkha. Californicus Motsch. (C. 18.) Sitkha.

Tachypus Megerle.

" elongatus Motsch. (Cat. p. 16.) Sitkha?
Bемвіріuм Latreille.

* glabriusculum Ménétr. (Cat. p. 15.) Sitkha.

Notaphus Megerle.

nigripes Kirby. (C. 19.) Sitkha.

Peryphus Megerle.

incertus Motsch. (C. 20.) Sitkha.

* cicatricosus Ménétr?(Cat.p. 11.)

Amer. aret. occ. fl. Kiwytsehpack.

brevis Motsch. (C. 21.) Sitkha.

planiusculus Mannerh. (A. 87.)

Sitkha.

(Kuprianovii Mannerh. (A.88.)

Sitkha.

ovipennis Motsch. (Bull. 1845̆.

IV. p. 352. Cat. p. 12.)

biimpressus Mannerh. (А. 89.)

Sitkha.

quadrifoveolatus Mannerh. (A. 90.) Sitkha.

fortistriatus Motsch. (C. 22.) Sitkha.

Tachys Ziegler.

"rivularis Ménétr. (Cat. p. 8.) Sitkha.

\section{DYTISCIA.}

Acilius Leach.

abbreviatus Eschsch. (A. 92) Sitkha.

Drtiscus Linné.

Ooligbukii Kirby. (C. 24.) Cont. Amer bor. occ. 
anxius Mannerh. (A.91.) Sitkha.

Colymbetes Clairville.

divisus Eschsch. (A. 93.) Sitkha. - in aquis stagnantibus frequens.

Inymes Erichson.

quadrimaculatus Aubé.(A. 9\%.) Sitkha. - iisdem locis vulgaris.

\section{Agabus Leach.}

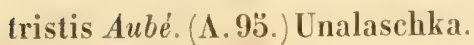

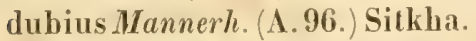
hypomelas Mannerh. (А. 97.)

Sitkha. - in aquis stagnantibus sat frequens.

scapularis Mannerh. (C. 25.)

Sitkha:

anthracinus Mannerh. (C. 26.)

Sitkha,

Hxproporos Clairville.

griseostriatus De Geer (A. 98.)

Unalaschka.

*oblongus Dej. (А. 99.) Unalaschka.

humeralis Eschsch. (A. 100.) Sitkha. - in aquis stagnantibus frequens.

contractulus Mannerh. (C. 27.)

Sitkha. ruficapillus Mannerh. (C. 28.) Sitkha. rufinasus Mannerh. (C. 29 . Sitkha. erythrostomus Mannerh. (C. 30.) Sitkha.
Hahipus Latreille.

pantherinus Aubé(C. 23.) Sitkha.

\section{GYRINIA.}

Gronus Linné.

picipes Eschsch. (A. 101.) Sitkha. - in lacubus et rivulis ubique vulgaris.

\section{HYDROPHILIA.}

\section{Helophonus Illiger.}

inquinatus Mannerh. (C. 118.) Sitkha.

*auricollis Eschsch. (A. 188.) Unalaschka.

\section{Cercyon Leach.}

limbatum Mannerh. (A. 189.) Sitkha. - in stercoratis et cadaveribus frequens.

fulvipenne Mannerh. (C. 119.) Sitkha.

fimbriatum Mannerh. (C. 120.) Edgecombe.

adumbratum Mannerh.(A.190.) Sitkha. - in stercore non infrequens.

posticatum Mannerh. (C. 121.) Sitkha.

\section{SILPHALIA.}

Necrophorus Fabricius.

(guttula Motsch. (C.91.) Sitkha. \{auripilosus? Eschsch. Dej. maritimus Eschsch. (A. 169.) Sitkha. - in cadaveribus frequentissimus. 
defodiens Mannerh. (B. 10.) Sitkha.

\section{Silpha Linné.}

Lapponica Herbst. (C. 92.) Contill. Amer. bor, oce.

Necrophimus Latreille. Hydrophiloides Eschsch. (A. 172.) Sitkha. - in cadaveribus frequens.

latus Eschsch. (C. 93.) Sitkha.

Sphaerites Duftschmid.

politus Mannerh. (B. 11.) Sitkhat.-in cadaveribus non infrequens.

Catops Fabricius.

Frankenhaeuseri Mannerh. (C. 94.) Sitkha.

Cadaverinus Eschsch.(A. 173.) Sitkha, - Cont. Amer. bor. occ. in cadaveribus vulgaris. Cryptophagoides Mannerh. (C. 95.) Sitkha.

\section{Colos Herbst.}

inermis Mannerh. (C. 96.) Sitkha.

Eutnei Stephens.

scitula Mäklin. (C. 90.) Sitkha.

Scydmanus Latreille.

Californicus Motsch. (C. 88.) Sitkha.

biformis Mäklin. (C. 89.) Sitkha.
PSELAPHIA.

Bryaxis Leach.

Albionica Motsch. (C. 178.) Sitkha.

Euplectus Leach.

parviceps Mäklin. (C. 180.) • Sitkha.

Tимитм Aubé.

clavicorne Mäklin. (C. 179.)

Sitkha.

\section{STAPHYLINA.}

Bolitochafa Mannerheim. notata Mäklin. (C. 31.) Sitkha.

\section{Tachyusa Erichson.}

fucicola Mäklin. (C. 32.) Ins. Edgecombe.

Homalota Mannerheim.

granulata Mannerh. (B. 1.) Unalaschka.

maritima Eschsch. (A. 102.)

Sitkha.

picipennis Mannerh. (A. 103.) Silkha.

laevicollis Mäklin. (C. 33.)

Sitkha.

cursor Mäklin. (C. 34.) Sitkha. nitens Mäklin. (C. 35.) Sitkha. moesta Mäklin. (C. 36.) Sitkha. pratensis Mälilin.(C.37.) Sitkha. geniculata Mäklin. (C. 38.) Ins.

Edgecombe.

planaris Mäklin. (C. 39.) Sitkha. 
breviuscula Mäklin. (C. 40.) tarsalis Mannerh. (A. 114.) Sitkha.

\section{Aleochara Gravenhorst.}

castaneipennis Sitliha. (A. 104.

C. 41.) Sitkha.

sulcicollis Mannerh. (A. 105.)

Sitkha.

cognata Mäklin. (C. 42.) Sitkha.

Taciunes Gravenhorst.

nigricornis Mannerh. (A. 106 . C. 43.) Sitkha.

frigidus Erichs. (A. 107. C. 44.)

Unalaschka; Sitkha.

circumcinctus Mäklin. (C. 45.)

Sitkha.

maculicollis Mäklin. (C. 46.)

Sitkha.

propinquus Mannerh. (A: 108.)

Sitkha.

elongatus Gyllenh. (A. 109.)

Unalaschka.

\section{Boletobius Leach.}

poecilus Mannerh. (C. 47.) Sitkha.

biseriatus Mannerh. (B. 2.)

Sitkha.

\section{Otwus Leach.}

macrocephalus Eschsch. (A. 110.

C. 48.) Sitkha.

\section{Staphylinus Linné.}

bicinctus Eschsch. (A. 113.) Sitkha. - in rebus animalibus vegetabilibusque putrefactis sat frequens.
Sitkha.

crassus Mannerh. (B. 3. C. 49.)

Unalaschka; Sitkha.

Philontuvs Leach.

Siegwaldii Mannerh. (A. 116.)

Sitkha. - in fimetis et cadaveribus frequens.

picipennis Mäklin. (C. 50.) Sitkha.

canescens Mäklin.(C. 51.) Ins. Edgecombe.

\section{Quedius Leach.}

plagiatus Mannerh. (A. 118. C. 52.) Sitkha. - sub cortice arbor, emortuar. sat frequens. longipennis Mannerh. (B. 4.) Unalaschka.

pediculus Eschsch. (A. 119.) Unalaschka; Sitkha.

erythrogaster Mannerh. (C. อ̈3.) Sitkha.

melanocephalusMannerh.(C.5̆4.) Contin. Amer. bor. occ.

brunnipennis Mannerh. (A. 120.) Sitkha. - in rebus putrescentibus minus frequens.

aenescens Mäklin.(C.5ว.) Sitkha marginalis Mäklin. (C. 56.) Sitkha.

molochinus Grav. (A. 121.) Sitkha.

*hyperboreus Erichs. (1. 122.) Unalaschka.

Stenus Latreille.

maritimus Motsch. (C. 57.) Sitkha. 
adspector Mäklin. (C.5̋8.) Sitkha. parallelopipedus Motsch. (C. 59.)

Sitkha.

cariniceps Mäklin. (C. 60.)

Sitkha.

brevipennis Mäklin. (C. 61.)

Sitkha.

Bledius Leach.

longipennis Mäklin. (C. 62.) Sitkha.

Oxytelus Gravenhorst.

fuscipennis Mannerh. (A. 123.)

Sitkha. - in fimo frequens.

Phloeonaeus Erichson.

biimpressus Mäklin. (C. 63.) Sitkha.

Srntomivar Curtis.

confragosum Mäklin. (C. 64.) Sitkha.

Anthophagus Gravenhorst.

laticollis Mannerh. (A. 124. C. 65.) Sitkha.

Heterops Eschscholtz.

(testaceus Eschsch. (A. 125.) \{ Sitkha. - in plantis locis paludosis vulgaris. ( ठ melanocephalus Motsch:

Arpedium Erichson.

maculicolle Mannerh. (A. 126.) Sitkha.

brevicolle Mäklin. (C. 66.) Sitkha.

\section{Lathrimaeom Erichson.}

subcostatum Mäklin. (C. 67.)

Sitkha.

(fimetarium Eschsch. (A. 128.)

Sitkha. - in fungis putri-

dis sat frequens.

fuscotestaceum Motsch.

\section{Omalium Gravenhorst.}

plagiatum Mannerh. (A. 127.) Sitkha.

strigipenne Mäklin. (C. 68.) Sitkha.

foraminosum Mäklin. (C. 69.) Sitkha.

exsculptum Mäklin. (C. 70.) Sitkha.

laesicolle Mäklin. (C.71.) Sitkha. segmentarium Mäklin. (C. 72.)

Sitkha.

longulum Mäklin.(C.73.) Sitkha. callosum Mäklin.(C. 74.) Sitkha:

Aлтновium Leach.

(Pothos Eschsch. (A. 129.) Sitkha. - in floribus speciei Pothos frequentissimum.

(ठ nigriventre Motsch.

Proteinus Latreille.

limbatus Mäklin.(C. 75.) Sitkha. basalis Mäklin. (C. 76.) Sitkha.

Megarturus Kirby.

pictus Motsch. (C. 77.) Sitkha. atratus Mäklin. (C. 78.) Sitkha. angulicollis Mäklin. (C. 79.) Sitkha. 


\section{9}

Micropeplus Latreille. costatus Mäklin. (C. 80.) Sitkha. brunneus Mäklin.(C.81.) Sitkha.

\section{TRICHOPTERYGIA.}

\section{Trichopteryx Kirby.}

laticollis Mäklin.(C.110.) Sitkha. insularisMäklïn.(C.111.) Sitkla. "Sitkaënsis Motsch. (C. 112.) Sitkha.

Ptenidium Erichson. pullum Mäkitin. (C. 113.) Sitkha.

\section{ANISOTOMIDA.}

Anisotoma Knoch.

Jateritia Mannerh. (C. 122.) Sitkha.

\section{Agxthidgm Illiger.}

angulare Mannerh. (C. 174.) Sitkha. (concinnum Mannerh. (C. 175.) Sitkha.

castaneum Ménétr.

rotundulum Mannerh. (C. 176.) Sitkha.

\section{PHALACRIDA.}

\section{Lxrochrus Erichson.}

brunnipennis Mannerh. (C. 173.) Sitkha.

\section{NITIDULARIA.}

Carpophilus Leach.

hemipterus Linné. (A. 174. C. 100.) Sitkha. dimidiatus Fabr. (C. 101.) Sitkha.

\section{Epuraea Erichson.}

convexiuscula Mannerh.(A.176.) Sitkha. - in floribus vulgatissima.

adumbrata Mannerh. (C. 103.) Sitkha.

ambigua Mannerh. (A. 177.) Sitkha. - sub cortice arbor. emortuar. minus frequens. truncatella Mannerh. (B. 12. C. 10\%.) Sitkha.

planulata Erichs. (C. 105.) Sitkha.

Crhocephitus Erichson.

*unicolor Motsch. (C. 102.)
Sitkha.

Ips Fabricius.

Dejeanii Kirby. (C. 99.) Sitkha.

Rhizophagus Erichson.

dimidiatus Eschsch. (A. 268. C. 160.) Sitkha.

scalpturatus Mannerh. (C. 161.) Sitkha.

Trogosita Olivier.

Mauritanica Linné. (A. 273) Sitkha.

pusillima Mannerh. (A. 274.) Sitkha.

\section{Peltis Geoffroy.}

Pippingsköldii Mannerh. (C. 97.) Sitkha. 


\section{0}

Peltastica Mamnerheim.

tuberculata Mannerh. (C. 98.) Sitkha.

\section{CeCUJICA.}

Cucusus Fabricius.

puniceus Eschsch. (A. 275.) Sitkha. - sub cortice pinorum emortuarum rarissime.

Peniacus Shuckard.

subcarinatus Mannerh. (C. 163.) Contin. Amer. bor. occ.

Laemophloeus Dejean.

longicornis Mannerh. (A, 276.) Sitkha.

Dendrophages Schönherr.

Cygnaei Mannerh. (B. 14.) Sitkha. - in truncis pini putridis exsiccatis rarissime.

Brontes Fabricius.

truncatus Motsch. (C. 164.) Sitkha.

Sinvanus Latreille.

dentatus Fabr. (C. 162.) Sitkha. (Surinamensis Linné. (A. 270.) S Sitkha.

frumentarius Fabr. sexdentatus Fabr.

CRYPTOPHAGA.

Cryptophagus Herbst.

octodentatus Müklin. (C. 106.) Sitkha. quadridentatus Mannerh. (A. 179.) Sitkha. - in fructibus exsiccatis et etiam extra domos non infrequens.

tuberculosus Mälkin. (C. 107.) Sitkha.

Atomaki Kirby.

fuscicollis Mannerh. (C. 108.) Sitkha.

fulvipennis Mannerh. (B. 13.) Unalaschka.

lepidula Mälilin.(C. 109.) Sitkha.

\section{LATHRIDIA.}

Corticania Marsham.

trisignata Mannerh. (C. 157.) Sitkha.

spinulosa Mannerh. (C. 158.) Sitkha.

Lathridus Illiger.

quadricollis Mannerh. (A. 263.) Sitkha.

protensicollis Mannerh. (A.266.) Sitkha.

sobrinus Mannerh. (C. 159.)

Sitkha

cordicollis Mannerh. (А. 267.)

Sitkha.

\section{DERMESTINA.}

Dermestes Linné.

vulpinus Fabr. (A. 180.) Sitkha.

\section{BYRRHIA.}

Brrphus Fabricius.

cyclophorus Kirby. (C. 114.)

Contin. Amer. bor. occ 
Morychus Erichson.

acuminatus Motsch. (C. 115.) Sitkha.

Simplocaria Marsham. nitida Motsch. (C. 116.) Sitkha.

Ampuicyenta Eschscholt: simplicipes Motsch. (C. 117.) Sitkha.

\section{CEUTHOCERA.}

\section{Ceuthocenus Schïppel.}

advena Schïppel. (A. 187.) Silkha.

\section{SCARABAEINA.}

Aphodius Illiger.

*Aleutus Eschsch. (A. 191.)

Unalaschka. guttatus Eschsch. (A, 192.)

Unalaschka.

Agratia Latreille. cylindrica Eschsch. (A. 19\%.) Unalaschka; Sitkha.

\section{BUPRESTINA.}

Buprestis Linné.

Langii Mannerh.(A.132.) Sitkha.

Melanophila Eschschollz. appendiculata Fabr. (C. 82.) Sitkha.

\section{EUCNEMIDIA.}

Epiphanis Eschscholtz.

cornutus Eschsch. (A. 13\%. C. 83.) Sitkha.

\section{ELATERINA.}

AтHovs Eschschollz.

ferruginosus Eschsch. (A. 15̈2.) Sitkha. - in domis et silvis vulgaris.

pallidipennis Mannerh. (A. 153.) Sitkha.

rufiventris Eschsch. (A. 15̈4.) Unalaschka.

Elater Linné, Eschscholtz.

carbonicolor Eschsch. (A. 142.) Sitkha. - in truncis putridis minus frequens.

Cryptonypus Eschscholtz.

littoralis Eschsch. (B. 5.) Unalaschka.

nocturnus Eschsch. (A. 138.) Sitkha.

limbatus Mannerh. (C. 84.) Contin. Amer. bor. occ. musculus Eschsch. (A. 139.) Unalaschka; Sitkha, in silvis rarissime.

\section{Conymbites Latreille.}

resplendens Eschsch. (A. 143.) Sitkha. - in truncis putridis sub cortice rarius.

spectabilis Mannerh. (C. 85.) Sitkha.

volitans Eschsch. (A. 146.) Sitkha. - sub cortice truncor. putresc. non infrequens. umbricola Eschsch. (A. 148.) Sitkha. - iisdem locis sat frequens. 


\section{2}

caricinus Eschsch. (B. 6.) Sitkha. - raro occurrit. Iobatus Eschsch. (B. 7.) Unalaschka.

Pristilophus Latreille. angusticollis Mannerh.(A. 144.)

Sitkha. - in truncis putridis sub cortice rarius.

sagitticollis Eschsch. (A. 147.)

Sitkha. - sub cortice arbor. emortuar rarius.

Dolopius Megerle. sellatus Mannerh.(C.86.) Sitkha.

\section{TELEPHORIDA.}

\section{Dictyopterus Latreille.}

hamatus Eschsch. (A. 155.) Sitkha. - in truncis pini rarius. simplicipes Eschsch. (A. 156.)

Sitkha. - iisdem locis parum frequentius.

Rhagonycha Eschscholtz. piniphila Eschsch. (A. 157.)

Sitkha. - in herbis et arbustis frequens.

sericata Mannerh. (B. 8.) Sitkha. binodula Mannerh. (B. 9.) Sitkha; Unalaschka.

Sinis Megerle.

pallida Eschsch. (A. 159.) Sitkha. - in arbustis et herbis sat frequens.

\section{CLERIA}

Thanasumos Latreille. abdominalis Kirby. (C. 87.) Sitkha.
Necrobia Latreille.

ruficollis Fabr. (B. p. 12.) Sitkha.

\section{P'TINIORA.}

\section{Privos Linné.}

Fur Linné (A. 167.) Sitkha.

Avoвium Fabricius.

paniceum Linné. (A. 166.) Sitkha. - in domis non infrequens.

\section{Cis Latreille.}

tridentatus Mannerh. (C. 154.) Sitkha.

biarmatus Mannerh. (C, 155.) Sitkha.

Americanus Motsch. (C. 1506.) Sitkha.

\section{BOSTRICHINA.}

Bostrichus Fabricius.

interruptus Mannerh. (C. 147.) Sitkha tridens Ménétr. (C. 148.) Sitkha. concinnus Mannerh. (C. 149.) Sitkha.

semicastaneusMannerh. (C. 150.) Sitkha.

septentrionis Leconte. (A. 261.) Sitkha. - sub cortice truncorum pini rarius. affaber Mannerh.(C.151.) Sitkha. nitidulus Mannerh. (A. 263. C. 152.) Sitkha. 
Xyloteres Erichson.

cavifrons Mannerh. (A. 260. C. 153.) Sitkha.

Hylesinus Fabricius.

sericeus Eschsch. (A. 256. C. 144.) Sitkha.

Hycurgus Latreille.

obesus Eschsch. (A. 257. C. 145. Sitkha.

Hylastes Erichson.

rugipennis Mannerh. (A. 258.) Sitkha.

nigrinus Mannerh. (C. 143.) Sitkha.

pumilus Mannerh. (A. 259. C. 146.) Sitkha.

\section{CURCULIONITA.}

\section{Rhyncolus Creutzer.}

brunneus Eschsch. (A. 255.) Sitkha. - in truncis putridis pini frequens.

\section{Sitophilus Schönherr.}

Oryzae Linné. (A. 253.) Sitkha.

Ceuthorhynchus Schïppel.

pusio Mannerh. (C. 142.) Sitkha.

Trachodes Schüppel.

Ptinoides Eschsch. (A. 249.)

Unalaschka; Sitkha, in domis rarius.

horridus Mannerh. (C. 140.) Sitkha. quadrituberculatus Motsch. (C. 141.) Sitkha.

EMphystes Mannerheim. fucicola Mannerh. (C. 139.) Ins. Edgecombe.

\section{Pissodes Germar.}

costatus Mannerh. (C. 138.) Sitkha.

Plinxues Germar.

carinatus Eschsch. (C. 137.) Sitkha

Leprous Germar.

gemellus Kirby. (C. 136.) Contin.

Amer. bor. occ.

Liophloeus Germar.

inquinatus Mannerh. (C. 135.) Sitkha.

Alophus Schönherr.

alternatus Say. (A. 244.) Sitkha. - in truncis pini rarius.

\section{Apion Herbst.}

cuprescens Mannerh. (A: 240.) Sitkha.

\section{RHINOSIMIDA.}

Tanyrhinus Mannerheim.

singularis Mannerh. (C, 133.) Sitkha

Salpivgus Illiger.

elongatus Mannerh. (C. 134.) Sitkha. 


\section{4}

\section{CERAMBYCINA.}

Spondylis Fabricius.

Upiformis Eschsch. (A. 277.) Sitkha. - sub cortice pinorum emortuar. rarius.

\section{Prionus Geoffroy.}

Californicus Motsch. (C. 165.) Sitkha.

Rosalia Serville.

funebris Motsch.(C.166.) Sitkha.

Opsimus Eschscholtz.

quadrilineatus Eschsch. (A. 279.)

Sitkha.

Pursocnemum Haldeman.

dimidiatum Mannerh. (B. 15.) Unalaschka.

Plegtnura Dejean.

spinicauda Eschsch. (C. 167.) Sitkha.

Ruagium Fabricius.

investigator Mannerh. (C. 168.) Sitkha.

Pachyta Megerle.

liturata Kirby. (C. 169.) Contin. Amer. bor. occ.

\section{CHRY SOMELINA.}

Donacia Fabricius.

Germari Eschsch. (A. 281. B. p 15. C 170.) Sitkha. flavipennis Eschsch. (A. 282.) Sitkha. - in gramine ad ripas lacuum cum praecedente ran rius.

SrNeta Eschscholtz.

carinata Eschsch. (A. 283.) Sitkha. - in silvis ligno pini recenter caeso insidens; rarius.

\section{Galemuca Geoffroy.}

luctuosa Mannerh. (C. 171.) Contin. Amer. bor. occ.

Gonoctena Chevrolat.

affinis Schönh. (C. 172.) Contin. Amer. bor. occ.

\section{COCCINELLIA.}

Coccinella Linné.

transversoguttata Say. (C. 177.) Contin. Amer. bor, occ.

\section{DIAPERIALIA.}

Phateria Latreille.

picta Eschsch. (A. 226.) Sitkha. TENEBRIONITA.

Cerandria Dejean.

cornuta Fabr. (C. 124.) Sitkha. maxillosa Fabr. (C. 125 ) Sitkha.

\section{Stene Kirby.}

(A. 227.)

Silkha. 


\section{5}

Heterophaga Dejean.

Mawritanica Fabr. (C. 123.) Sitkha.

\section{Tenebrio Linné.}

molitor Linné. (C. 128.) Sitkha.

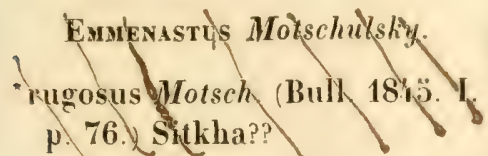

\section{SERROPALPIA.}

Dincaea Fabricius.

Holmbergii Mannerh. (C. 126.) Sitkha.

Stexotrachelus Latreille.

obscurus Mannerh. (C. 129.) Confin. Amer bor. occ.

\section{PYROCHROIDA}

Рхтно Latreille.

Sahlbergii Mannerh. (A. 23\%.

C. 127.) Sitkha.

Pogonocends Fischer.

Ephemeroides Ménétr. (C. 130.) Sitkha.

\section{MORDELLONA.}

Anaspis Geoffroy.

sericea Mannerh. (A. 237. C. 131.)

Sitkha.

pallescens Eschsch. (G. 238 .) Sitkha.

\section{CANTHARINA.}

\section{Mecoë Linné.}

strigulosus Mannerh. (C. 132.)

Ins. Kodiak. 



\section{E R R A T A.}

pang. 298. lin. 21. discribuntur - lege: describuntur.
- 300. - 1. Pippings-
- - 2. kioll
pipping-
sköld
- 348. - 16. rum
mum
- - 23. Pippings-
- - - 24. liöld
Pipping- sköld
- 350. - 20. Pippings-
Pipping-
- - - 21. köld
slïld
- 3อ̆1. - 9. tia interdum subele- vata,
tio interdum subele- vato,
- 33̈6. - 15. niger, atro-hispidus,
- $\quad$ - 25. trunculorum
- 357. - 17. Sitkha,
- 361. - 5. Sitkha
- - - 17. lectus.
nigra, atro-hispida, truncorum
Sitkhae
Sitkhae
lecta.
subrotundatum.
acuto
exstantem Anisotoma
(LEIOdeM) Castaneam
(LEIODES) castanea
- 383. - 19. Agralia
Aegialia 



\section{DRITTER NAGITRAG}

Z.UR

\section{KAEFER - FAUNA}

\section{DER NORD - AMERIKANISCIIEN LAENDER DES RUSSISCHEN REICHES,}

von

$$
\text { Graf C.G. Mannerheim, }
$$

Doctor der Philosophie und beider Rechte, Präsident des Kaiserl. Hofgerichtes zu Wiburg. Grosskreuz des St. Wladimir-Ordens zweiter Klasse, des St. Annen-Ordens erster Klasse mit der Kaiserl. Krone und des St. Stanislaus-Ordens erster Klasse, mehrerer Akademien der Wissenschaften und gelehrten Gesellschaften Mitglied.

\section{MOSCAU。}

In Der Buchdruckerei der Katserlicien Universitat 1853. 
(Extrait du Bulletin de la Sociëlé Impériule des Naturalistes de Moscou, année 1853. 슬 5 )

џұенсорь, Н. М. Сиезиеев. 


\section{DRITTER NACIITRAG}

ZUR

\section{KAWWER - FAUVI}

DER NORD - AMERIKANISCHEN LAENDER DES RUSSISCHEN REICHES,

von

Graf C. G. Mannerietm.

Als ich im vorigen Jahre den zweiten Nachtrag zu dieser Käfer-Fauna herausgab, konnte ich kaum denken, dass ich bald darauf ein noch beträchtlicheres Material zu einem dritten erhalten würde. Der in dem vorausgeschickten Werke bereits erwähnte Conducteur II. J. Holmberg kehrte nehmlich im letztvergangenen Herbste, von seinen Reisen, in die Heimath glücklich zurück und brachte ausserordentlich reiche, mit unermüdetem Fleisse gewonnene Sammlungen in allen $\mathrm{Zweigen} \mathrm{der} \mathrm{Zoologie} \mathrm{mit,} \mathrm{unter} \mathrm{welchen} \mathrm{doch}$ die entomologischen gewiss den Vorrang behaupten und auf eine ausgezeichnete Weise bezeugen, was ein so viel- 
seitig gebildeter Naturforscher und Sammler, wie Herr Holmberg, zu leisten im Stande ist.

Im Herbste 1849 trat Herr Holmberg in Gesellschaft des Herrn Philosophiae Magisters Fr. Franlienhaeuser, jüngeren Bruders des in meinem zweiten Nachtrage rühmlichst erwähnten Doctors A. Frankenhacuser, auf einem aus Abo abgesegelten Schiffe, seine Reise an. Das Ziel derselben galt Californien, das märchenhafte Goldland, wo Holmber, und Frankenhacuser, die beide sich dem Studium des Bergwesens gewidmet hatten, ihre erworbenen Kenntnisse in diesem Fache, mit gutem Erfolge in Anwendung zu bringen holften. Allein schon in dem ersten Hafen Valparaiso, begegneten ihnen sicherere Nachrichten aus Californien, als die sie bei der Abfahrt aus Europa erhalten hatten, über die vielen Schwierigkeiten, mit denen eine vortheilhafte Anstellung bei den Gold - Ausbeuten verknüpft sei. Diese Nachricht, im Vereine mit den unglaublich hohen Frachtpreisen von Valparaiso nach San Francisco, bewogen unsere Reisenden, auf demselben Schiffe, das nach Sitkha bestimmt war, nach der Hauptniederlassung der Russisch Amerikanischen Compagnie abzusegeln, um daselbst eine directe Gelegenheit nach San Francisco abzuwarten. Auf diese Weise gelangten sie, am 10-ten April 1850, in NeuArchangelsk auf der Insel Sitkha an.

Um das, was ich schon in dem zweiten Nachtrage über die Naturverhältnisse der Insel Sitkha erörtert habe, zu ergänzen, will ich hier noch Einiges aus den Mittheilungen, die ich von dem Herrn Holmberg erhalten habe, über ihre geognostische Beschaffenheit hinzufügen. Diese Insel ist. 
sowohl wegen der Wildheit der Eingebornen als wegen der Unzugänglichkeit ihres von Morästen und Urwäldern bedeckten Innern, noch wenig ermittelt. Die hauptsächliche Gebirgsart scheint Thonschiefer zu sein, der durch mehr oder weniger Einmengung von quarzigen Bestandtheilen, theils sehr schiefrig, theils fast ungeschichtet, auftritt und endlich, durch vulkanischen Einfluss, in eine harte feinkörnige Grauwacke übergeht, die von $\boldsymbol{E}$. Hofmann "Kieselgrauwacke» genannt wird. Der vulkanische Einfluss wird durch das Auftreten von Graniten und Syeniten verdeutlicht. Auf der NO - Seite der Insel steht tertiäre Kohle mit Pflanzenabdrücken an. Im Westen der Insel Sitkha erhebt sich, auf der Insel Krusow oder Edgecombe, der ausgebrannte Vulkan Edgecombe, dessen Krater eine verwitterte thonige Lava umgiebt. Der grösste Theil dieser Insel scheint aus basaltiger Lava und Trachyt zu bestehen.

Schon im Mai begann, auf der Insel Sitkha, die entomologische Ausbeute unserer beiden Reisenden, und zwar unter Steinen; sie war in dieser Jahreszeit hauptsächlich auf Carabica beschränkt, die auch den ganzen Sommer hindurch, unter ähnlichen Verhältnissen, vorkamen. Einen ebenfalls reichen entomologischen Ertrag gewährten, etwas später, die in der Nähe der Festung gefällten, so wohl alten als frischen Bäume, unter deren abgeschälter Rinde die Käfer-Seltenheiten der Gegend, als Alophus alternatus Say, Plectrura spinicauda Eschseh., Salpingus elongatus Mannerh. etc. anzutreffen waren. An warmen Abenden des Juli konnten beim Sonnenuntergange auch einiges im Fluge gesammelt werden. Die an Gebüschen und Gräsern reiche 
Vegetation Sitkhas leidet gänzlichen Mangel an WiesenBlumen, ausser Becrenblüthen, doch verlieh der Köscher hin und wieder etwas, wie IIeterops testaceus Eschsch., Anthobium pothos Eschsch., Rhagonycha piniphila Eschsch., Silis pallida Eschsch., Ceutorhynchus pusio Mannerh. u. m. d. Schon im August nehmen die Käfer bedeutend an Menge ab, und im September konnten nur noch solche in verfaulten Pilzen erbeutet werden. Auf der Insel Edgecombe beherbergte der Fucus, der sich auf dem feinen Sande des Meeresufers angesammelt hatte, mehrere seltene Käfer, als Nebria Mannerheimii Eschsch., Philonthus canescens Mäkl., Emphyastes fucicola Mannerh. etc. Aehnliche Verhältnisse gaben aber auf der Insel Sitkha keine Ausbeute, abgerechnet den Staphylinus crassus Mannerh., der in einigen Exemplaren unter Fucus vorkam.

Am 6-ten Januar 1851 gingen unsere Reisenden nach San Fransisco, allein nach einem nur zweiwöchentlichen Aufenthalte in dem damals ordnungslosen Californien begaben sie sich nach den IIawajischen oder Sandwich Inseln und brachten fünf Wochen in der reichen, doch leider Käfer - armen Tropennatur der Insel Oahu zu. Am 17-ten April langten sie wieder in Sitkha an, in der Absicht, den Sommer in entfernteren Gegenden der Russisch - Amerikanischen Kolonien zuzubringen. Diese Absicht wurde auch bald erfüllt und es sind hauptsächlich den demzufolge unternommenen Excursionen, denen wir die vielen interessanten neuen Entdeckungen im Gebiete der Coleopterologie verdanken, welche ich zur weiteren Vervollständigung der Russisch - Amerikanischen Küfer - 
Fauna in der gegenwärtigen Abhandlung systematisch beschreiben werde.

Auf dieser Reise kamen die Herren IIolmberg und $\boldsymbol{F r}$. Frankenhaeuser in der Bai Woskresensk auf der Ostseite der Halbinsel Kenai am 16-ten IIai an. Die Bai erstreckt sich 30 englische Meilen ins Innere der Halbinsel und zeigt in ihren steilen von Nadel- und Laubholz (Espen) bewachsenen Ufern einen ziemlich harten Thonschiefer, von vielen Quarzgängen durchzogen. Zwei mächtige Gletscher, der eine im Innern der Bai von Nordost kommend, der andere bei der Mündung derselben von Westen streichend, schieben ihre Moränen bis an die Oberlläche des Wassers. Die wenigen Tage (vom 16-ten bis 19-ten Mai), die Herr Holmberg in der Bai von Woskresensk zubrachte, waren, bei der frühen Jahreszeit, in entomologischer Hinsicht noch wenig ergiebig, doch wurde, gegen Ende des Monats ziemlich viel von Hern Frankenhaeuser gesammelt, der, vor seiner Bergwanderung über die Halbinsel, hier länger verweilte. Die Reisenden trennten sich an diesem Orte, für den ganzen Sommer, um im Herbst wieder auf der Insel Kadjak zusammenzutreffen. Herr Frankenhaeuser schloss sich jetzt einer Expedition an, die im Innern von Kenai geognostische Untersuchungen anstellen sollte. Zuvor hielt er sich, bis zum 30-sten Mai, an der Bai selbst auf, wo sein Lagerplatz an der Mündung des Flusses Thkujaktnú war. Dann zog er, etwa 15 Werst längs dem Ufer dieses Flusses, zum See Thkujabná und von hier, in den ersten Tagen des Juni, einen steilen, waldbewachsenen, auf den Gipfeln noch schneebedeckten, gegen 3 bis 400 Fuss hohen, 


\section{8}

in Ost und West auslaufenden Bergrücken hinan, der die Wasserscheide der Gewässer des Kenaischen Meerbusens und der Baien Woskresensk und Tschugatsch bildet. Am 6-ten Juni stieg er in das breite Thal des, aus einem Gletscher entspringenden Flusses Tschunitén hinab, dessen Lauf er etwa 20 Werst nach Norden bis zu seinem Ausflusse in den See Skeljamná verfolgte. Dieses Thal ist grösstentheils mit Laubwald, darunter viel Birken, bewachsen. Hierauf ging die Reise auf dem Sec Skeljamná und hernach auf dem Flusse Skeljauktuu, in westlicher Richtung, bis zur Mündung des Flusses Tschunuktnú. Von hier wandte er sich nach Norden längs des Ufers des letztgenannten Flusses, der, ungefähr 8 Werst vor seiner Mündung, einen prachtvollen Wasserfall, von fast 300 Fuss, in drei Absätzen bildet. Das Thal des Tschunuktnú möchte, nach Hern Frankenhaeusers muthmasslicher Berechnung, 30 bis 40 Werst lang und 3 bis 4 Werst breit sein. An dasselbe stossen, unter rechten Winkeln, die schmalen Thäler seiner zahlreichen Nebenflüsse. Das Hauptthal ist mit Nadel-und Laubholz bewachsen, wie auch das untere Gebiet der Nebenflüsse. Das obere Gebiet der letzteren, so wie die dieselben begränzenden Berge zeichnen sich aber durch reichen Graswuchs besonders aus. - Die höheren spitzen Berggipfel, die etwa auf 2000 Fuss relativer Höhe zu schätzen sind, treten vollkommen kahl hervor. Wie gross die absolute Höhe des Tschunuktnú - Thales sei, ist von Herrn Frankenhaeuser nicht angegeben worden, jedoch muss sie, nach dem starken Fall der Gewässer zu urtheilen, namentlich im ohern Theile des Thales, wo der Reisende sich vom 17-ten 
Juni bis zum 15-ten Sept. aufhielt, nicht gering sein. Am letztgenannten Tage zog Herr Frankenhaeuser sich zurück zum Skeljanktnú und an dem folgenden bis zu dessen Mündung in den See Bentilent. Am Flusse Skeljanktnú befindet sich die einzige Ansiedelung der Kenaizer (Thnaina) auf der ganzen Strecke zwischen der Bai Woskresensk und der Nicolajewschen Redoute am Kenaischen Meerbusen. Die Seen Bentilent und Kastudelenbna (zusammen nur einen See bildend) vereinigen alle Gewässer der obenerwälınten Wasserscheide und führen sie durch den Fluss Kaktnú (") dem Kenaischen Meerbusen zu. Vom See Kastudelenbna an gewinnt das Land, welches bis dahin sehr bergig war, ein anderes Ansehen, indem es nach Westen hin vollkommen flach und mit reichen Nadel - und Laubholz - Waldungen bewachsen ist. Eine Fahrt von etwa $\mathbf{8 0}$ Werst auf dem Kaktnú brachte die Expedition, am 19-ten September, in die Nicolajewsche Redoute, wo Herr Frankenhaeuser sich nach Kadjak einschiffte.

Nachdem Herr Holmberg, am 19-ten Mai, sich von seinem Reisegefährten getrennt hatte, begab er sich erst nach der Insel Chtagaluk, wo in der Bai Nutschek die Konstantinovsche Redoute angelegt ist. Obgleich er fünf Tage in der Redoute verweilte, wobei er auch entferntere Excursionen vornahm, war doch das Frühjahr noch zu wenig vorgeschritten, als dass er eine grössere Käferausbeute hätte machen können. Zu den häufiger dort vorkommenden

(") Es ist derselbe Fluss, der in meinem zweiten Nachtrage, nach einer fehlerhaften Angabe des Herrn Doctor A. Frankenhaeusers, iuberall "Kaknu" benannt wird, cin Irrthum, den ich also hier berichtige. 
Arten gehören einige Bostrichus und Hylurgus rufipennis Kirby. So viel Herr IIolmberg die Gebirgsart der Insel (das Innere derselben ist auch hier noch unzugänglich) in ihren Felsentblössungen am Ufer beobachten konnte, so besteht sie nur aus Thonschiefer verschiedener Varietäten. Am 26-ten verliess er Nutschek, segelte schon am 2-ten Juni in den Meerbusen von Kenai hinein, konnte aber erst am 6-ten unter der Nicolajewschen Redoute am Ausflusse des reissenden Kaktnú - Stromes ankommen. Die Redoute liegt an dem rechten erhabenen Ufer des Flusses, das hier gleichsam einen Sandwall, auf grünem Thon ruhend, bildet. Das linke Flussufer ist eine niedrige grasbewachsene Ebene, entstanden durch allmählige Versandung der Barre. Anstehendes Gestein konnte Herr Iolmberg in der ganzen Gegend nicht wahrnehmen. Herrliche Birkenwälder umgeben die Redoute und mannigfache Blumenpracht schmiickt die Wiesen der Umgegend. - Nlit Recht verdient also dicser Theil der Halbinsel die ihm gegebene Benennung des Blumengartens der Kolonien. Daher ist diese Gegend an Käfern auch die reichste von allen im Gebiete des Russischen Antheiles von Nord-Amerika. Fast drei Wochen brachte Herr IIolmberg dort zu, konnte aber eigentlich nur die erste Woche dem Sammeln widmen, indem später, bei wärmerer Witterung, Myriaden Mücken und Moskitos sich entwickelten, die buchstäblich den Sammler zı verzehren drohten. Er hatte schon früher mit diesen Plage-Thierchen in Lappland Bekanntschaft gemacht, doch in dieser Beziehung duldet, nach seiner Erfahrung, Lappland mit Kenai keinen Vergleich. Und obschon die Witterung in der ersten 
Woche weniger günstig war, brachte er doch während derselben eine fast eben so grosse Menge Küfer zusammen, als er das ganze Jahr vorher, in Sitkha und den nachfolgenden Sommer in Kadjak sammelte. Abgerechnet das hohe Ufer der Redoute, wo unter Steinen, im Fluge. im Grase, auf Blumen, in und unter Holz Käfer häufig vorgefunden wurden, gewährte das niedrige Sandufer eine reiche Fundgrube derselben. Unter dem fast senkrechten Uferwalle breitet sich eine Ebene von feinem Meeressande aus, die wahrscheinlich auch einer allmähligen, obgleich hier noch keinesweges factisch nachgewiesenen Hebung des Landes ihre Entstehung verdankt. Auf dieser Ebene vertreten oft riesenhafte, ausgeworfene Baumstämme die sonst in diesen Gegenden häufig vorkommenden Ufergerölle und FucusAblagerungen (*), und diese Baumstämme sind ein Zufluchtsort für verschiedene Käfer, so wie' mancherlei Celien, Cryptohypnus littoralis Eschsch., Trachodes Ptinoides Eschsch. etc. Viele Vertiefungen dieses Ufers bilden durch Regen und Schneemassen oft ansehnliche Wasserlachen, in denen es von Dytiscien wimmelt und an deren Rande niedliche Elaphrus - und Peryphus - Arten auf Raub lauern. Zur Zeit der Ebbe wird meilenweit das Sandufer trocken gelegt und beim Sonnenscheine lassen sich die Käfer dort nieder, um auf dem nachgebliebenen Schlamme Nahrung zu suchen. Unter diesen erscheinen ofters alte Bekannte aus unserem Europaeischen Norden, wie: Silpha Lapponica, Bromius Vitis, Lina Lapponica und Coccinella trifasciata.

(*) Auch an Meeres-Conchylien Jeidet dieses Ufer gänzlichen Mangel. 
Südlich von nächsterwähnter Redoute tritt am Cap Neniltschik und, noch südlicher, in der Bai Kotschekmaksk die Tertiärformation in grossen Braunkohlen-Ablagerungen auf. Auf das, der Redoute gegenüber liegende Land erheben sich zwei mächtige vulkanische Kegel. Der südliche: die Iljaminskaja (Sopka d. h. Spitze) ist 12,066 Fuss hoch und raucht seit Menschengedenken; Feuer aber wird nicht wahrgenommen, nur die aufsteigende Rauchsäule zeigt die Richtung des Windes an. Der andere, die Gorjälaja oder Wyssokaja, 11,270 Fuss hoch, ist ausgebrannt; doch bestehen unter den Eingebornen Gerüchte, dass auch dieser vor nicht sehr langer Zeil geraucht haben soll (*).

(") Es ist diese Nordische Inselgegend, so zu sagen, mit Vulkanen und heissen Quellen ïbersäet. Da es vielleicht von Intresse sein dürfte, dieselben zu kennen, will ich sie hier so vollständig wie möglich angeben :

Wales oder Biber-Insel: Vulkan Kalder. 1775 thätig? (Don Antonio

Maurelle.).

Insel Sitkha oder Baranow: Heisse Quellen.

Insel Krusow oder Edgecombe: Vulk. Edgecombe. fälschlich 1796 von Hofmann als thätig angegeben.

Festland von Nord-Amerika: Vulk. Irillor.

Vulk. Gutwetterberg.

Vulk. St. Elias.

Küstenvulkan an der Bai Tschugatsch.

Vulk. Wrangell. 1819 entdeckt, thä-

tig. Jährlich mebrere Erdbeben.

Vulk. Gorjälaja oder Wyssokaja(hoher Berg).

Vulk. Iljamna, rauchend.

Halbinsel Aljaska: Vulk. Wenjaminow. $1830-1840$ rauchend (Wenjaminow.).

Heisse Quellen an der Perenossny-Bucht. 
Am 29-ten Juni erreichte IIerr IIolmberg den Pawlowschen Hafen auf der Insel Kadjak, seinen Bestimmungsort für den Sommer. Er sollte die Insel in Baidarken umschif-

Vulk. Pawlowsky. $1762-1786$ thätig. 1786 schliesst sich einer der Kratern (Chamisso); 1790 thätig (Sarytschew) gegenwärtig noch rauchend.

Vulk. Medwednikowsky. 1790 thätig (Sarytschew).

Vulk. Morschowsky.

Heisse Quellen auf einer kleinen Insel an der Einfahrt in die Bai Morschowsky.

Ausserdem mehrere heisse Quellen.

Vulkaninsel Amak, thätig im vorigen Jahrhundert; seit 1804 ruhig (Krusenstern.).

Insel Unimali. Vulk. Khaginak. zu verschiedenen Zeiten rauVulk. Schischaldin. $\{$ chend und feuerspeiend mit Vulk. Pogromnaja. Aschenfall und Erdbeben.

Insel Akun. Vulk. Akun. 1828 periodische Rauchsäulen (Postels).

Heisse Quellen.

Insel Akutan. Vulk. Akutan. 1790 rauchend (Sauer und Sarytschew);

1828 rauchend (Postels).

Heisse Quellen.

Insel Unalaschka. Vulk. Makuschkin. Zu verschiedenen Zeiten rauchend und feuerspeiend mit starken Erdbeben.

Heisse Quellen.

Insel Umnak. Vulk. Tulikskoi. zu verschiedenen Zeiten rauchend Vulk. Sewidowsky. $\{$ und feuerspeiend mit AschenVulk. Retscheschnoi.) fall und Erdbeben.

Heisse Quellen.

Vulkaninsel Bogoslow entstand den 7-ten Mai 1796 durch Eruption und Erdbeben, hat seit 1823 zu rauchen aufgehört und bedeckt sich schon allmählig mit Vegetation.

Heisse Quellen.

Insel Kigamiljach mit einem Vulkane. Früher Eruptionen; 1828 Erdbeben.

Heisse Quellen.

Insel Tanach-Angunach mit einem Vulkane, der 1828 thätig war. (Luitke).

Heisse Quellen.

Inseln Uljagan und Tschegulak mit vulkanischen Kegeln, die früher thätig gewesen sein sollen. 
fen, um dem Gouverneur von Sitkha einen Bericht ïber deren geognostische Beschaffenheit abzustatten. Aus dieser Untersuchung hat sich im Allgemeinen ergeben, dass die

Insel Junaska mit einem Vulkane. 1817 rauchend (Choris); 1824 neue Eruptionen (Liitke); 1830 Aschenauswürfe.

Insel Amuchta mit einem Vulkane. 1786 ganz in Flammen (Schelechow); 1790 thätig (Sarytschew); 1830 ruhig (Liitke).

Insel Siguam mit einem Vulkane. Vor 1790 in Thätigkeil(Sarytschew); 1827 rauchend (liitke).

Schlammkratere und heisse Quellen (Luitke).

Insel Atkha. Vulk. Sarytschew. Vulk. Kljutschewsky.

Vulk. Korowinsky. abwechselnd thälig.

Vulk. die Konische Sopka.

Vulk. die Sergejewsche Sopka.)

Schlammkratere und heisse Quellen.

Insel Konjuschy. Angeblich in beständiger Hebung begriffen und 1827 in der Mitte rauchend (Liitke).

Insel Kasatotschy mit erloschenem Krater.

Insel Gross - Sitchin: der Vulk. 1760 ruhig (Tolstych); 1792 feuerspeiend (Sarytschew); $\mathbf{1 S 2 9}$ rauchend und schneebedeckt (Ingenstrïm).

Insel Adach: der weisse Vulkan. 1760 thätig(Tolstych, Baikow); 1784 thätig (Sarytschew).

Spätere Nachrichten fehlen.

Heisse Quellen.

Insel Kanaga mit einem Vulkane, in dessen Krater 1763 Schwefel gesammelt wurde; 1786 feuerspeiend (Schelechow); 1827 rauchend (Lütke).

Heisse Quellen.

Insel Tanuaga mit einem Vulkane. 1763-1770 beständig thätig; 1791 rauchend (Sauer).

Insel Gorjäloj mit einem Vulkane. 1760 thätig (Baikow); 1792 feuerspeiend (Sarytschew); 1829 rauchend (Ingenström).

Insel Semisopotschny, rauchend.

Insel Sitignak, 1776 feuerspeiender Berg (Bragin).

Heisse Quellen.

Insel Klein-Sitchin mit einen Vulkane. 1828 rauchend (Lülke). 
Insel Kadjak hauptsächlich aus Thonschiefer besteht, mehr oder weniger durch vulkanischen Einfluss verändert. An den Endpunkten seiner Umschiffung der Insel, d. h. im Nordosten, in der Bucht Kanichogoluk und im Siidwesten, in der Nähe der Ansiedelung Kaschjukwagmjut beobachtete Herr IIolmberg Granit, der den Thonschiefer gehoben und aus seinen horizontalen Schichten geworfen hatte und welcher der Analogie zufolge am N. und NIV.- Ufer der Insel in grösseren Massen vorkommen möchte. Das Cap Tonkoi besteht aus einem tertiären, versteinerungführenden Sandsteine. Nur der östliche und nordöstliche Theil der Insel ist mit Nadelwald bewachsen. Je mehr man nach Süden und Südwesten vorschreitet, um so mehr nehmen diese Waldungen ab; in der Ansiedelung Kaschjukwagmjut sieht man hin und wieder nur einige verkrüppelte Pappeln. Dahingegen sind die Buchten und Bergschluchten der Insel mit einem üppigen Graswuchse ausgestattet, der die prächtigsten Weideplätze liefert. Dessenungeachtet hat Herr Holmberg, obschon auch hier die Blumen im Sommer kaum geringer sind als in Kenai, keinen Käfer, weder im Grase noch im Fluge gefangen, ausgenommen einen Phytonomus, den er auf abgepfliickten Himbeeren wahrnahm. Auf der Nordkiiste der Insel will man das Vorschreiten der Bewaldung nach Westen beobachtet haben, eine Erscheinung, die sich vielleicht durch anhaltende Richtung des Windes zu gewissen Jahreszeiten, erklären liesse.

Von der Insel Afognak hat Herr IIolmberg nur das südliche Ufer in seiner ganzen Ausdehnung besucht. Es ist stark bewaldet und besteht aus schon früher erwähntem 
Thonschiefer, jedoch von vielen Quarzadern und Gängen durchzogen. Die Ausbeute an Insekten war dort nicht bedeutend, lieferte indessen doch etliche an anderen Orten nicht gefundene Käfer-Arten.

Am 18-ten October gelangten unsere Reisenden wieder beide zusammen nach Sitkha, von wo aus sie am 17-ten December die Rückreise nach Europa antraten.

Dieser Bericht, den ich nach den gefälligen Mlittheilungen des Herrn IIolmbergs abgefasst habe, dürfte vielleicht etwas weitliuftig erscheinen; allein eine Darstellung der Umstände und Verhältnisse, unter welchen die Käfer-Ausbeute gemacht wurde, die das llaterial zu einer beträchtlichen Ergänzung der Käfer-Fauna der Nord-Amerikanischen Länder des Russischen Reiches geliefert hat, möchte wohl hier ihren Platz gewissermassen vertheidigen können. Es ist meines Erachtens vom höchsten Gewicht, um Beobachtungen über die geographische Verbreitung der Insekten anstellen zu können, auch die geognostischen und vegetativen Verhältnisse der Länder, in welchen sie vorkommen, näher und mit einer gewissen Vollständigkeit zu kennen. Um das entomologische Publicum auch mit der Geographie der Länder, deren Käfer den eigentlichen Gegenstand meiner schriftlichen Bearbeitungen hier ausmachen, lasse ich eine Karte mit dieser Abhandlung folgen, die ich ebenfalls dem Herrn IIolmberg zu verdanken habe, indem er sie mit seiner auch in diesem Fache geschickten Hand ausgeführt hat.

Ausser der beträchtlichen Käfer - Ausbeute der Herrn 
IIolmberg und Fr. Frankenhaeuser, die nicht weniger als 18000 Exemplare enthält und mir bei gegenwärtigem Nachtrage mit einer Freigebigkeit zu Gebote stand, wofür ich diesen beiden Entomologen meinen ganz besonderen Dank öffentlich abzustatten verpflichtet bin, wurde mein jetziges Unternehmen auch durch andere meiner entomologischen Freunde, aufs erfreulichste begünstigt. IIerr Collegienrath $E$. Ménétriés hatte nemlich die Güte, mir alle aus den Ländern des Bezirkes meiner Fauna in Museum der Kaiserlichen Akademie der Wissenschaften zu St.Petersburg befindlichen Käfer, in sofern sie durch meine frühere Beschreibungen noch nicht bestimmt waren, zur Untersuchung und descriptiven Behandlung mitzutheilen. Unter diesen zeichnet sich eine von dem Herrn Sagosskin, Officier der Kaiserlichen Russischen Marine, der in den Jahren 1842 - 47 den Fluss Kwichpakh des Nord-Amerikanischen Continents bis nach Nulato bereiste und noch höher vordrang, gemachte kleine Sammlung ganz besonders aus. Er hat nemlich manche, in der Richardson schen Fauna von Kirby, aus der Expedition des später unglücklichen Franklins nach dem Mackenzie-Flusse, bekannt gemachte, ausgezeichnete Species wieder gefunden, die als vorzügliche Seltenheiten das Museum der Akademie jetzt schmücken. Gleichfalls hat Herr Obrist-Lieutenant von Motschulsky mir die Gefälligkeit erzeigt, mir alle die in seiner, an russischen Käfern reichen Sammlung, vorhandenen Arten aus den Gegenden, deren Behandlung in Kïferfaunistischer Hinsicht ich unternommen habe, zuzuschicken; theils solche, die aus diesen Ländern stammend, in seinen 
vielen entomologischen Schriften angegeben worden sind, theils solche, dic noch unbeschrieben und nirgends erörtert waren, nur mit Ausnahme seines Cybocephalus unicolor, den er nicht hat wiederfinden können und er schlechterdings ein Cereyon zu sein glaubt, weswegen derselbe, möglicher Weise, mit irgend einer der von mir bereits beschriebenen Cercyon-Arten zusammenfallen möchte. Und schliesslich theilte mir Herr Doctor R. Ferd. Sahlberg die wenigen Species mit, welche aus seiner auf der Insel Sitkha gewonnenen Käfer-Ausbeute bisher noch unbeschrieben geblieben waren; allein dieser kleine Beitrag zu meiner Fauna war um so mehr wichtig, als er mich eine ächte Melasomen-Art, obschon von ganz eigenthümlicher, höchst paraloxer, Gestalt kennen lehrte, die einzige die von dieser, sonst in anderen Gegenden der Welt, und gleich in dem nicht zu sehr entfernten Californien vielartigen Gruppe, bis jetzt in den Russisch - Amerikanischen Ländern angetroflen worden ist, weswegen ich auch sogar erst in Frage stellte, ob nicht hier ein Irrthum in der Angabe des Fundortes obwalten künne, da aber Doctor Sahlberg mir auf das bestimmteste versichert, dass er sich noch sehr wohl erinnere, wo und wie er diesen sonderbaren Käfer genommen, musste folghich demselben ein Platz in dieser Fauna eingeräumt werden. Pflichtgemäss bezeuge ich daher den Herren Ménétriés, v. Motschulsky und Sahlberg hier meine Erkenntlichkeit für ihre freundschaftlichen Beiträge zur Vervollständigung meiner Arbeit, die eben dadurch jetzt Ansprüche machen dürfte, eine systematische Auscinandersetzung aller Käfer-Arten zu enthalten, welche aus den 
fraglichen Gegenden bisher in den Europäischen Sammlungen sich vorfinden.

Bei der zufolge dieser Mittheilungen jetzt staltgefundenen genauen Prüfung der Typen zu einigen von Ilerrn v. Motschulsky in seinen Katalog der Russischen Käfer eingerückten Carabica, kann ich es nicht vermeiden hier zu erklären, dass Nebria macrocephala Motsch. nichts anders ist als Nebria stigmula Dej. ( ${ }^{*}$, dass Platysma alternata Motsch. als nur ein unreifes verkrüppeltes Individuum von Bothriopterus orinomum Leach. sich ergiebt, dass Agonothorax planipennis Motsch. als von Anchomenus parumpunctatus Fabr. nicht im geringsten verschieden betrachtet werden kann, und dass Agonothorax Anchomenoides Motsch. nicht von Anchomenus Mannerheimii (**) Sahlb. Dej. (morio Gebler, welchen Herr v. Molschulsky nicht nur für besondere Art hält, sondern auch sogar in eine andere Gattung Limodromus [Cat. p. 70.] unterbringt.), als ebenfalls Agonothorax splendidulus Motsch., wie ich schon nach der Angabe des Herrn Baron $v$. Chaudoirs bemerkt habe, nicht von Anchomenus impressus Pans., zu trennen sind. Herr v. Motschulsky hatte nemlich alle diese Species in einer vom verstorbenen Eschscholtz stammenden kleinen Sammlung vor vielen Jahren zu Dorpat erworben, die überhaupt

(") Eine Hochalpin-Form, die gewiss der geologischen Beschaffenheit ron Unalaschka fremd sein müsste.

(**) Dass dieser unter Kieferrinde lebende Käfer auf der waldiosen Insel Unalaschka vorkommen sollte, ist an und für sich schon etwas ganz widernatuirliches. 
aus Küfern von den Reisen dieses berïhmten Naturforsehers bestand; es war gleichwohl keine Unmöglichkeit, dass ir dieselbe auch Europäer sich eingeschlichen hatten und zu einem gewaltigen Irrthum Veranlassung geben konnten, den man sich hiiten muss, noch weiter zu reproduciren, weshalb schon IIerr $v$. Motschulsky selbst bei diesen seinen vermeinten neuen Arten meistentheils den Fundort mit einem Fragezeichen angegeben hat. Was ferner seinen Emmenastus rugosus betrifft, so kann ich jetzt aus Autopsie ermitteln, dass dieses Thierchen nichts anderes ist als ein Blapstimus, dem pulverulentus Eschsch. nahe stehend. Ich besitze von der nemlichen Art Exemplare aus Nord - Californien, wo sie häufig vorkömmt und woher das MotschulsFyssehe Stiick auch wahrscheinlich stammt, um so mehr da er ebenfalls für diesen Käfer ein Fragezeichen bei "Sitkha» angebracht hat. Die von ihm gemachte Vergleichung seiner für denselben errichteten neuen Gattung mit Gnathosia und Zophosis hält also nicht Stich. Aus meinem, dem zweiten Nachtrage beigefügten Verzeichnisse sind daher zu streichen: Nebria macrocephala Motsch., Bothriopterus alternatus Motsch., Anchomenus planipennis Motsch., Anchomenus Motschulskii Mannerh. (Anchomenoides Motsch.), CybocephaTus unicolor Molsch. und Emmenastus rugosus Motsch. Die in demselben Verzeichnisse vorkommenden: Stenolophus quadripunctatus Ménétr. befindet sich hier unten als Varietät des St. axillaris Ménétr.; Celia compacta Motsch. und Peryphus cicatricosus Ménétr. aber sind als Synonymen anderer bereits bekannten Species auf ihren Stellen eingerückt, und Nebria carbonaria Eschsch. ist wieder, als auf 
einem Irrthume beruhend, gegen den neuen Namen $N$. bifaria vertauscht worden.

Nach diesen Ausmusterungen enthält vor der IIand die Käfer-Fauna der Nord-Amerikanischen Länder des Russischen Reiches systematisch charakterisirte und beschriebene Carabica 114, Dytiscia 35, Gyrinia 1, Ilydrophilia 13, Silphalia 25, Pselaphia 3, Staphylina 106, Trichopteryyıa 5, Anisotomia 10, Phalacra 1, Nitidularia 20, Cucujica 8, Cryptophaga 15, Lathridia 17, Dermestina 2, Byrrhia 6, Ceuthocera 1, IIcterocera 1, Scarabaeina 6, Buprestia 4, Eucnemia 1, Elateria 30, Cyphonia 1, Telephora 7, Cleria 2, Ptinia 7, Bostrichina 20, Curculionia 21, Rhinosimia 3, Cerambycina 19, Chrysomelina 12, Coccinellia 5, Diaperialia 1, Tenebrionia 6, Serropalpia 4, Oedemeria 1, Pyrochrota 3, Anthicia 1, Mordellona 2 und Cantharina 1, oder im Ganzen zusammen 5 h0 Species. Ich halbe in diesem dritten Nachtrage bei den Beschreibungen die, bereits in dem, dem zweiten beigefügten Verzeichnisse beobachtete Reihenfolge des natürlichen Systens angenommen. Wenn ich schon in gedachtem Verzeichnisse den Namen der Familien eine neutrale Beendigung zu ertheilen mir erlaubte, könnte diess wohl gewissermassen die Iferrn Entomologen befremden. Ich hielt es aber für besser, den Versuch zu machen, eine bestimmte Consequenz hierin einzufïhren, und nicht, wie es bis jetzt in dieser Beziehnng gewöhnlich geschah, Masculina, Feminina und Neutra unter einander zu mischen. Coleopterum, Colcoptera sind ja schon neutrius generis, warum sollten denn auch nichl die Familiennamen es ebenfalls sein mil einer adjectiven En- 
dung, die sich auf das Nomen: Coleopterum beziehen würde. Die Finalen auf $i d a$, ides hat ja schon einer der berühmtesten Entomologen unserer Zeit als, wegen der aus dem Griechischen hergeleiteten Namen anstössig und oftmals unpassend, gerügt, obgleich er später selbst von seinem ausgesprochenen Grundsatze mehrere Abstecher gemacht hat. Ich habe es aber genau zu vermeiden gesucht, und mich auf das Gewissenhafteste bemüht, diese Namen so $\mathrm{zu}$ bilden, dass das Lateinische und das Griechische nicht in eines zusammengefoltert wurde, und berücksichtigte gleichzeitig, überall wo es nöthig sein konnte, die Regel der griechischen und lateinischen Sprachen bei den aus jener entnommenen Wortbildungen. Indem ich die Namen so kurz wie möglich abfassen wollte, habe ich auch, seit meinem Verzeichnisse bei dem vorigen Nachtrage, einige Veränderungen zu machen mir erlaubt, wie aus dem Vorhergehenden im Vergleiche mit diesem Verzeichnisse erhellt.

Nach den Beschreibungen der hier unten charakterisirten Gattungen und Arten habe ich es für unumgänglich gehalten, zu berichten, welche Species namentlich auch in anderen Localitäten des fraglichen Bezirkes, als es schon bei den Beschreibungen angegeben wurde, gefunden worden sind. Es geschieht natürlicher Weise in der Absicht, ihre geographische Verbreitung vollständiger zu bestimmen. Was aber im Allgemeinen die Verbreitung der in den Russischen Ländern von Nord-Amerika angetroffenen Käfer betrint, so ist sie bei manchen eine selır grosse. Wir finden hier wieder recht viele Arten aus dem Norden von Europa schon längst bekannt und die ihre Heimath über 
ganz Sibirien ausdehnen, andere wieder mit den Erzeugungen des übrigen Nord - Amerikanischen Festlandes gemeinschaftlich $\left(^{\star}\right)$. Die Silpha Lapponica gehört unter jene und lebt überall im Gefolge der ichtyophagischen Völker innerhalb des ganzen nördlichen Polar-Cirkels von Lappland

(') Um eine nähere Kenntniss von den Erscheinungen der geographischen Verbreitung der Käfer, besonders im Norden, zu gewinnen, weise ich auf eine vom Herrn Philos. Cand. $F$. W. Mäklin neulich herausgegebene Akademische Abhandlung hin. Sie ist, im Mai dieses Jahres, zu Helsingfors erschienen in schwedischer Sprache unter dem Titel: Bidrag till kännedom om insekternas geografiska utbredning i norden, med hufvudsakligt afscende pa Skandinaviens och Finlands Fauna. Diese in manchen Stücken, so wie durch vielseitige Beobachtungen und Aufschlïsse höchst anziehende Arbeit verdiente wohl bald in eine allgemeiner verbreitete Sprache iibersetzt zu werden. Ilerr Maklin ertheilt uns darin eine Darstellung über die geographische A usloreitung nicht nur dex Insekten ins Besondere, sondern auch der übrigen ThierClassen iberhaupt, von Osten nach Westen, welche Ausbreitung doch nicht in einem directen Verhältnisse zu den Breite-Graden des Lri- balls steht, weil sie sich mehr nach den isothermischen Linien oder Curven richtet und nach der aus Osten nach Westen statufindenden Lage der Berge der alten Welt auch noch mehr an Bedeutenheit gewinnt. Je höher gegen Norden, wo die Berge an Höhe abnchmen und eine mehr gleichförmige Natur der sich mehr und mehr annihernden Länder der alten und neuen Welt, welche zuletzt nur durch cine Meerenge getrennt sind, darbictet, tritt die Gemeinschaftlichkeit der organischen Formen fast wic eine Regel auf. Im Allgemeinen deutet Herr Mäklin eine auch gewiss nicht zu läugnende Neigung der thierischen Organismen, sich vom Osten nach Westen zu verbreiten, an, dessen immerwährendes Fortfahren er ebenfalls behauptet. Ich wïde auch noch hinzufïgen, dass selbst auch die Wanderungen des Menschengeschlechts diese Neigung periodisch gewissermassen getheilt und dass eben unsere Finnländische Völkersehaft denselhen Wegr zu ihrer Einwanderung in die von ihr jetzl bewohnten Liader eingeschlagen hat, inden der berühmte, uns leider zu friih cntriichte Professor Castrén, seiner elhnographischen Forschungen zafolisa, schon bewies, dass dieses Volk in seinen nachherigen weitin Vorbreitungen ursprüglich von den Altaischen Bergen herab gewandert war. 
bis Grönland. Hauptsächlich gilt diese Gemeinschaftlichkeit der Arten mit dem Europäischen Norden das zu den Russischen Besitzungen gehörende feste Land von NordAmerika und beziekt sich weniger auf die Inseln, die eine mehr eigenthümliche Käfer - Fauna darbieten, gewiss als eine natürliche Bedingung ihrer geognostischen Verhältnisse zufolge der vulkanischen Einflüsse. Allein auch die Formen der auf den Inseln vorkommenden Käfer stellen, bei ganz wenigen Ausnahmen, einen Nord-Europäischen Typ heraus und nähern sich dadurch auf eine augenscheinliche Weise den übrigen in diesen Schriften verhandelten coleopterologischen Erzeugnissen. Es ist daher oft eine recht schwierige Aufgabe, mit systematischer Gewissheit die Arten als selbstständig zu begründen, indem manche mit einer der bereits bekannten Europäischen ungemein nahen Verwandschaft hervortreten und doch nicht mit diesen sich als specifisch identisch füglich vereinigen lassen. Dieser Umstand hat meine Arbeit in vielen Stücken um ein Bedeutendes erschwert; ich habe aber eine möglichst strenge Kritik zu beobachten gesucht und hoffe daher wenigstens zu nicht allzu grossen Missgriffen Veranlassung gegeben zu haben.

Wie schon oben gesagt, enthält meine Käfer-Fauna bis jetzt 540 Species. Zwar sind noch viele Theile ihres Bezirkes in entomologischer Hinsicht gänzlich unerforscht geblieben, unter anderen die grosse Halbinsel Aljaska, die aller Wahrscheinlichkeit nach eine nicht geringe Ausbeute liefern würde. Bei alle dem und weil Aljaska auch ähnliche vulkanische Verhältnisse mit den benachbarten Inseln 
darbietet, ist es zu vermuthen, dass ihre coleopterologischen Productionen denen dieser Insel fast ganz gleich sein müssen. Was bisher entdeckt und ausgemittelt ist, muss also, bei sehr wenigen noch zu erwartenden Ergänzungen, meine Käfer-Fauna so ziemlich der beabsichtigten Vollständigkeit derselben schon sehr nahe heranrücken.

Wie in dem vorigen Nachtrage ist Herr Candidat Mäklin mir auch in diesem mit den Beschreibungen der Staphylina und etlichen anderen kleinen Käfer behülflich gewesen, für welchen freundschaftlichen Beistand ich ihm hier meine aufrichtigste Dankbarkeit bezeuge. Er hat ausser den von den Herrn Ménétriés, v. Motschulsky, IIolmberg und Fr. Frankenhaeuser dazu gelieferten Materialien, auch Gelegenheit gehabt, eine kleine Sarnmlung Käfer zu erwerben, die Herr Pharnaciae Candidat $A$. Collan von seiner Reise nach den Russisch-Amerikanischen Kolonien mitbrachte und die einige von anderen Entomologen nicht gefundenen Arten und Varietäten enthält, wie es sich aus dem Folgenden erweist.

Willnäs, den 1 Juli, 18 3.

\section{A R A B I C A.}

1. Elapirus obliteratus: obscure rufescenti-cupreus, opacus; capite confertim punctato, inter oculos profunde longitudinaliter rugoso; thorace capitis latitudine, punctatissimo, lateribus antrorsum rotundato, obsoletissime foveolato, in ipso medio tenuissime 
canaliculato; elytris disperse et minute punctulatis, maculis obscurioribus impressis vix conspicuis, fere evanescentibus, ad suturam utrinque areis quatuor in seriem dispositis et insuper extra illas quinta postica obliqua, laevigatis nitidis, forma irregularibus.

\section{Longit. 5 lin. Latit. 2 lin.}

Ad portum Pauli insulae Kadjak, in quisquiliis ripae lacustris, initio Augusti, a D. Holmberg rarissime lectus.

E. Lapponico Gyllenh. quodammodo similis, sed multo major, latior, minus convexus, thurace lateribus magis rotundato-dilatato, minus profunde et confertim punctato et elytrorum sculptura fere omnino deleta ab illo praeterea facile dignoscendus.

2. Elapurus gratiosus: supra obscure aeneus, hinc inde virescenti-micans, punctatissimus, subtus violaceo - conflagratus; thorace capite angustiore, lateribus rotundato, dorso vix foveolato, in ipso medio tenue canaliculato, canalicula antrorsum triangulariter biramosa; elytris maculis violaceis impressis ocellatis quadruplici serie, interpositis seriebus tribus ex areolis cupreis laevigatis nitidissimis; pedibus viridibus, femoribus tibiisque basi ferrugineis.

Var. b. supra cupreo-aenea; capite viridi-consperso; thorace ad latera posterius viridi-maculato; subtus virescens; pedibus et sculptura ut in $a$.

Longit. $3 \frac{1}{4}$ lin. Latit. $1 \frac{1}{9}$ lin.

Habitat ad ostia fl. Kaktnu peninsulae Kenai, locis humidis mense Junio frequens; D. Holmberg.

EL. nipario Linné nonnihil similis, sed magis elongalus, thorace angustiore et elytris longioribus imprimis diversus. 


\section{$27 \mathrm{llg}$}

3. Tracil Pachú S Holmberg I: supra cupreo-aeneus nitidissimus, subtus nigro - piceus, capite thoraceque laevissimis; hoc transverso, longitudine duplo latiore, lateribus antrorsum vix dilatato, margine laterali anguste reflexo, medio tenuissime canaliculato, basi transversim depresso, utrinque ad angulos posticos fovea magna profunda, plica elevata extrorsum limitata; elytris in medio subtilissime striato - punctulatis, lateribus et longe ante apicem omnino laevigatis; antennis nigro - piceis; pedibus obscure rufescentibus.

Longit. 2 lin. Latit. $\frac{3}{4}$ lin.

Ad ostia fl. Kaktnu peninsulae Kenai individuum unicum a D. Holmberg detectum.

A Tr. (Blethisa) Zetrenstedti Schönh. differt capite thoraceque omnino laevigatis, illo fronte aequali, haud impresso, hoc latiore, breviore, angulis posticis rectis, elytris longioribus, disco tantum et subtilissime striato punctulatis, antennisque obscurioribus.

4. Pelophica Eschscholtzil Sturm : Mannerheim Bullet. de la Soc. de Moscou. 1843. p. 190. 20.

Habitat etiam in insula Kadjak, locis humidis sub lapidibus, mense Julio frequens; D. Holmberg.

Var: $b$. elytris totis rufo - castaneis.

Longit. 5 lin. Latit. 2 lin.

Mannerh. in Hummel Essais entomol. III. p. 40. 4. Var. b. Specimen a D. Eschscholtz, in insula Unalaschka captum, in museo meo asservatur.

Var. c. supra obscure cupreo-aenea, elytris margine viridi-cupreo, pedibus piceis, femoribus basi rufo - castaneis.

Longit. $4 \frac{1}{2}$ lin. Latit. 2 lin. 
Ad castellum Nicolajevsk peninsulae Kenai, sub la' ' 'ibus in arena litorali a D. Holmberg minus frequenter $i$ i ita.

Var. d. supra cupreo-aenea, magis metallic resplendens, in interstitio elytrorum sexto foveis quatuor impressis, pedibus rufis, genubus tibiarumque basi et apice obscurioribus.

Longit. 5 lin. Latit. 2 lin.

In insula Kadjak simul cum Var, a a D. IIolmberg lecta.

5. Nebria bifaria : nigra nitida, femoribus rufis; thorace brevi, transverso, cordato, posterius valde angustato, angulis rectis, disco transversim striguloso, basi punctulato, margine laterali late explanato, obsoletius rugoso; elytrorum striis profunde exaratis, punctatis, interstitio tertio foveolis quatuor vel quinque, quinto duabus vel tribus profunde impressis.

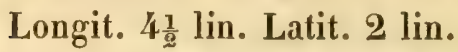

Nebria carbonaria Mannerh. Bullet. de la Soc. de Mloscou. 1852. pag. 293. 4.

Habitat in insula St. Pauli; D. Wosnesensliy. Individua e Kamschatka etiam obtinui, quo in errorem inductus ad N. carbonarium retuli. N. carbonaria vera, cui D. Ménétriés nomen vulcanicolae imposuit, mihi hodie cognita, quare descriptio N. carbonarias a me olim facta e Fauna Boreali-Americana eradenda.

6. Crcinus reticulatus Motschulsiy: Mannerh. Bullet. de la Soc. de Moscou, 1852. p. 292. 1.

Typum in museo D. Motschulsky asservatum, mecum amice communicatum, accuratius examinavi. Species certe distincta. Color totus niger; statura migis convexa, imprimis autem capitis et thoracis fi mat omnino aliena a C. marginato Eschsch. mox ds 'in- 


\section{9}

1.) Cendus. Caput nempe aculissime tricarinatum, intra r.urinas profunde excavatum. Antennae, praesertim articulo primo, validiores. Thorax multo minor, valde cordatus, supra convexiusculus, postice sinuatim valde coarctatus, parte constricta subquadrata, angulis basalibus rectis, margine anguste elevato postice plicato, disco profundius canaliculatus, antice posticeque profunde transversim impressus. Pedes multo robustiores et longiores.

7. LORICERA CONGESTA: supra obscure aenea, elytris aeneo-castaneis, subtus nigra; thorace longitudine sesqui latiore, lateribus valde rotundato, posterius nullo modo sinuato, sed margine ibi reflexo, medio canaliculato, basi et intra marginem lateralem posterius profunde punctato, angulis posticis rotundatis, intra illos utrinque profunde foveolato, foveola in sulcum arcuatum antrorsum continuata, in disco ante medium utrinque foveola oblonga impressa; elytris disco trifoveolatis, apicem usque profunde punctatostriatis, striis valde approximatis et creberrime punctatis; antennis nigro-piceis, tibiis tarsisque rufo-testaceis.

Longit. $3 \frac{3}{4}$ lin. Latit. $1 \frac{1}{2}$ lin.

Habitat in peninsula Kenai, secundum D. Ménétriés, qui individuum e Museo Imp. Academiae Scient. Petrop. ad describendum mecum benevole communicavit.

Forma et sculptura thoracis elytrorumque striis valde approximatis et creberrime apicem usque punctatis ab omnibus hujus generis speciebus jam cognitis recedit.

8. Loricena SEMIPUNCTAta Eschscholtz: Mannerh. Bullet. de la Soc. de Moscou. 1843. p. 191. 25. 40

ग2i Longit. $3 \frac{3}{4}$ lin. Latit. $1 \frac{1}{2}$ lin. 


\section{$\therefore$. 30}

Etiam ad ostia 1. Kaktnu peninsulae Kenai a D. Holmberg capta. Occurrit minus frequens, exitu mensis Junii, sub lapidibus ad litera maris.

Diagnosi jam datae suppleatur: a L. PiLiconni Fabr. cui valde affinis, differt thorace postice minus et haud rotundatim angustato, elytrisque longioribus, magis linearibus et supra deplanatis striisque subtilius punctulatis.

9. Loricera decenPUNCTATA Eschscholtz: Mannerh. Bullet. de la Soc. de Moscou. 1843. p. 192. 26.

Var. b. major, thoracis basi creberrime confertim punctata; vix vero species diversa.

Longit. 4 lin. Latit. $1 \frac{1}{2}$ lin.

Habitat in insula Kadjak sub lapidibus sat frequens; occurrit haec species etiam ad sinum Nutschek insulae Chtagaluk rarius; D. Holmberg.

10. Dyschirius transmarinus Ménétriés: Oblongus, supra obscure cupreo-aeneus, subnitidus, subtus nigro-piceus; mandibulis, antennis pedibusque obscure rufo-piceis; tibiis anticis spinis tribus longis introrsum curvatis interne armatis, extus vix dentatis; thorace suborbiculato, basi apiceque truncato, medio canaliculato; elytris thorace sesqui latioribus, ovalibus giblis, sat profunde striatis, striis prima et secunda usque ad apicem integris, ceteris pone medium evanescentibus, omnibus antice profunde punctatis.

Longit. $1 \frac{5}{6}$ lin. Latit. $\frac{2}{3}$ lin.

Motschulsky, die Käfer Russl. I. p. 18.

Habitat in insula Sitkha, teste D. Ménétriès. Mus. Acad.

Imp. Scient. Petrop. 


\section{$31 / 23$}

Statura et magnitudo fere D. onscon Sahlb. Gyllenh. colore cupreo - acneo, thorace minus orbiculato, elytris paullo longioribus, angustioribus et tibiis anticis spinis longioribus armatis diversus.

11. Dyscin I IUS FRIGI DUS: elongatus, supra aeneo-cupreus, nitidissimus, subtus niger; thorace latitudine longiore, lateribus parum rotundato, dorso minus convexo, in medio canaliculato; elytris oblongo-ovatis, thorace vix latioribus, antice punctato - striatis, striis mox pone medium evanescentibus; antennis nigris; femoribus nigro-aeneis nitidis; tibiis anticis spinis tribus longis, introrsum curvatis armatis, extus inermibus.

Longit. $1 \frac{2}{3}$ lin. Latit. $\frac{1}{2}$ lin.

Individuum unicum ad fl. Tchunitén peninsulae Kenai, sub lapide, initio Junii, a D. F. Frankenhaeuser captum.

12. HARPALU FULVILABRIS: oblongus, niger; thorace brevi, longitudine latiore, subquadrato, posterius parum angustato, in medio evidenter canaliculato, basi utrinque obsolete foveolato, subtiliter, circa foveas confertim, punctulato, angulis omnibus rotundatis; elytris striatis, maris nitidis, feminae opacis, apice oblique truncatis, vix sinuatis, interstitio tertio postice punctis duobus impressis; ore cum mandibulis, palpis et labro, thoracis elytrorumque limbo anguste, eorum margine inflexo, antennis pedibusque rufis.

Var. b. labri medio infuscato, thorace paullo latiore, foveis basalibus obsoletioribus; cetera omnino ut in $a$, quare ut species diversa aegre dijudicanda.

Harpalus subaeneus Motschulsky in litteris.

Longit. $4-4 \frac{1}{2}$ lin. Latit. $1 \frac{2}{3}-2$ lin. 
In ora orientali insulae Kadjak, mense Julio, sub lapidibus a D. Holmberg rarius lectus. Var, b a D. Motschulsliy communicata, in eadem insula quoque capta.

II. zimвato Duftschmid affinis, sed differt thorace posterius angustiore, angulis magis rotundatis, dorso profundius canaliculato, labro, mandibulis elytrorumque margine inflexo rufis.

13. Harpíus curtatus: breviusculus, parallelus, niger, nitidus, palpis, antennis brevibus pedibusque rufo-ferrugineis; thorace brevissimo transverso, longitudine duplo latiore, laevi, lateribus aequaliter modice rotundato, angulis etiam omnibus rotundatis, basi utrinque foveolato, foveis impunctatis; elytris striatis, interstitio tertio impunctato.

Longit. $3 \frac{3}{4}$ lin. Latit. $1 \frac{2}{3}$ lin.

Habitat ad sinum Woskresensk peninsulae Kenai, medio Junii, sub lapidibus rarius; D. F. Frankenhacuser.

Praecedente multo brevior, praeterea toto margine laterali concolore, antennis capitis cum thoracis dimidio vix longioribus, thorace breviore latiore, impunctato elytrorumque interstitio tertio omnino impunctato ab hac specie mox dignoscitur.

14. Ac upalpus axylaris Ménétriés: oblongus, niger, nitidus; capite tantum supra oculos utrinque disperse punctato; thorace elytris dimidio angustiore, subquadrato, lateribus rotundato, posterius nonnihil angustato, angulis oblique truncatis obtusis, dorso canaliculato, utrinque profunde foveolato, foveis punctulatis; elytris striatis; antennarum articulo primo toto, saepe proxime insequentibus basi, elytrorum basi plerumque anguste, sutura et margine, genubus tibiarumque apice rufis. 
Stenolopius axillaris Motschulsky, die Käfer Russl. I. p. 22.

$V a r . b$ obscurior, antennis magis nigris, elytrorum margine basali et sutura angustius rufescentibus.

Stenolopius euadripuxctatus Ménétriés : Molschulsky, die Käfer Russl. I. p. 22.

Var. c. elytrorum basi et sutura latius tibiisque totis rufo-ferrugineis.

$$
\text { Longit. } 2-2 \frac{1}{4} \text { lin. Latit. } \frac{5}{4}-1 \text { lin. }
$$

Habitat in insula Kadjak sub lapidibus sat frequens; D. Holmberg. Specimina ibidem capta e Museo Acad. Imp. Scient. Petrop. etiam amice communicavit I). Ménétriés. Var. $b$ in insula Sitkha inventam sub nom. Stenolophi guadripunctati Ménétr. misit D. Motschulsiy. Var. $c$. ad sinum Nutschek insulae Chtagaluk, initio Junii, locis humidis, sub lapidibus parcius a D. $110 \mathrm{~lm}$ berg capta.

15. Acupalous longuesculus: elongatus, nigro-piceus, nitidus; capite in vertice remote punctato, fronte foveis duabus punctatis; thorace subruadrato, lateribus modice rotundato, posterius vix angustato, angulis obtusis, dorso canaliculato, apice et basi obsolete punctulato, postice utrinque leviter foveolato; elytris striatis, interstitio tertio puncto impresso; antennarum articulo primo, secundi et tertii basi, thoracis limbo late pedibusque ferrugineo-testaceis; elytris lividotestaceis, utroque plaga longitudinali ante medium incipiente, fusca, interdum virescenti-micante.

Longit. $2-2 \frac{1}{4}$ lin. Latit. $\frac{2}{3}-\frac{3}{4}$ lin.

Ad sinum Woskresensk peninsulae Kenai, medio Maji, sub lapidibus frequens; D. Holmberg. 
16. ACUPALUS CONFLA RATUS: oblongus, nigro-piceus nitidus; capite in vertice remote punctato; thorace subruadrato, postice subangustato, angulis ohtusis, doreo canaliculato, basi utrinque evidentius foveolitto, for eis punctulatis; elytris striatis, interstitio terlio puncto minuto impresso; antennarum articulo primo, tertii basi thoracisque limbo, antice et postice late et lateribus anguste rufo-ferrugineis; pedibus obscure testaceis, femoribus infuscatis; elytris rufo-testaceis, utroque plaga ante medium incipiente, nigro - fusca.

Longit. 2 lin. Latit. 1 lin.

IIabilat ad ostia fl. Kaktnu peninsulae Kenai, medio Junii, sub lapidibus minus frequens; D. Holmberg.

Pracedenti quoad colores simillimus, sed multo, praesertim in elytris, brevior, praeterea thorace antice laevigato, basi profundius foveolato pedibusque obscurioribus ab illo imprimis distinguendus.

17. Argutor linearis: elongatus, sublinearis, niger nitidus; thorace elytrorum latitudine, longitudine angustiore, posterius rotundatim parum angustato, angulis obtusiusculis, in medio tenue canaliculato, postice utrinque unistriato, striola basi profunde punctata; elytris linearibus, apice ipso rotundatis, simpliciter evidenter striatis punctisque tribus impressis.

Longit. 3 lin. Latit. 1 lin.

Ad ostia f1. Kaklmu peninsulae Kenai a D. IIolmberg semel captus.

18. Onaseus rufiscapus: oblongus, sublinearis, niger, subnitidus, antennarum articulo primo, femorum basi corumgue et libiarum apice interiore rufis; tho- 
race subquadrato, lateribus vix rotundato, basi parum angustato, angulis obtusis, medio canaliculato, postice utrinque bistriato et intra angulos punctato, striola externa brevissima obsoleta; elytris oblongis, tenue striatis, striis subtiliter punctulatis, interstitio tertio punctis quatuor impressis.

Longit. 5. lin. Latit. 2. lin.

Habitat in insula Kadjak sub lapidibus rarius; D. IIolmberg.

Ab O. anturacino Illig. Dej. differt imprimis thorace multo breviore, striola baseos interiore longiore, evidentiore, carinula exteriore deficiente, elytris multo levius striatis et antennarum articulo primo rufo.

19. Botiriopterus SEXPUCtatus: oblongus, niger nitidus; thorace elytris vix angustiore, longitudine latiore, lateribus valde rotundato, postice parum angustato, utrinque striato, intra angulos punctato, angulis obtusis, medio canaliculato; elytris brevioribus, oblongo-ovatis, striatis, striis obsolete punctatis, utroque foveolis sex, quarum duae in stria secunda, quatuor in tertia, impressis.

Longit. $5-5 \frac{1}{2}$ lin. Latit. $2-2 \frac{1}{3}$ lin.

In insula Kadjak per totam aestatem ubique vulgaris; in insula Afognak rarius sub lapidibus, mense Augusto; D. Holmberg.

Pterosticho adstricto Eschsch. (Mannerh. Bullet. de la Soc. de Moscou. 1843. p. 203. 59.) valde affinis, sed major, latior, thorace latiore, postice minus angustato, ad angulos crebrius et fortius punctato elytrisque sexpunctatis praecipue diversus.

20. Cryobius hy erbored Ménétriés: oblongus, con- 


\section{+\$ 36}

vexus supra viridi-aeneus, nitidus, sublus niger; thorace elytris angustiore, latitudine breviore, lateribus valde rotundato, postice modice angustato, angulis subobtusis, basi utrinque foveis oblongis binis impresso, exteriore duplo breviore, ibique subruguloso, medio canaliculato, disco obsolete undulatim strigoso; elytris valde convexis, profunde striato-sulcalis, striis punctulatis, interstitiis subcarinatis, tertio punctis quatuor impressis; antennis pedibusque nigro-piceis.

Longit. $4 \frac{1}{5}$ lin. Latit. $1 \frac{5}{4}$ lin.

Motschulsky, die Käfer Russl. I. p. 54.

IJabitat in insula St. Georgii; D. Wosnesensliy. Specimen descriptum a D. Motschulskiy benevole transmissum.

A sequente differt praesertim colore, thorace breviore, angulis posticis obtusis et elytris profundius striatosulcatis.

21. Cryobius subexaratus: oblongus, convexus, supra nigro-aeneus, nitidus, subtus niger; thorace elytris angustiore, latitudine vix breviore, subquadrato, postice sinuato - angustato, angulis subrectis, basi utrinque stria arcuata impunctata profunde impressa, medio canaliculato, disco obsolete undulatim strigoso; elytris valde convexis, profunde striatosulcatis, striis subtiliter punctatis, punctis quatuor impressis; antennis pedibusque nigro-piceis, illarum articulo primo summo basi rufescente.

Var. b. supra caeruleo-aenea.

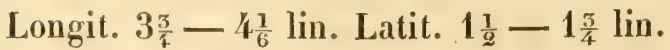

In insula Linalaschka a D. Cygnaeo captus; individua indidem et ex insulis Kurilis allata etiam communicavit D. Ménétriès. 


\section{$37 \quad / 29$}

Lum Pterosticho ventricoso Eschsch. (Mannerh. Bullel. de la Soc. de Moscou. 184.3. p. 20\%. 61.) a me hucusque confusus, reapse antem ut species diversa dignoscendus, si examini accuratiori subjiciatur; vextmeoso a Cel. Eschscholtz dato nempe semper minor, angustior, convexior, thorace angustiore, longiore, postice lateribus magis sinuato-angustato, angulis basilibus subrectis, impressionibus posticis laevibus, elytris valde convexis, profunde striato-sulcatis, striis subtiliter punctatis praeterea bene distinctus.

22. CRуовiUs Vixdicitus: oblongo-ovatus, supra cupreo-aeneus, nitidus, subtus niger, thorace latiludine breviore, subquadrato, posterius nodice lineariattenuato, medio canaliculato, basi utrinque profunde unistriato impressionibus impunctatis, angulis posticis fere obtusis; elytris leviter striatis, striis subtiliter obsolete punctatis, punctisque tribus vel quatuor inpressis; antennarum articulo primo basi rufo; pedibus nigro-piceis, trochanteribus femorumque summa basi rufescentibus.

\section{Longit. $4 \frac{3}{4}$ lin. Latit. $1 \frac{3}{4}$ lin.}

Var. b. supra nigra, nitida; antemnarum articulo primo basi femoribusque rufis; thorace minore et elytris angustiore quam in $a$; elytris quam in illo profundius striatis interstitiisque magis elevalis: vix vero species diversa?

Longit. $4 \frac{1}{3}$ lin. Latit. $1 \frac{\mathrm{g}}{3}$ lin.

Ilabitat sub lapidibus in oris meridionali et occidentali insulae Kadjak, mense Julio, sat frequens; 1). Iolmberg. Var. b. semel tantum iisdem locis obvia.

Differt a Cr. ventricoso (Pтerosticuo Mannerh. I. c.) Eschsch., cui aflinis, thorace paullo angustiore, postice 


\section{$\$ 38$}

parum attenuato (non ut in illo sinuatim angustato), strigulis undulatis dorsualibus plane nullis, impressionibus posticis fere omnino laevibus, angulis basalibus obtusis nullomodo prominulis, et elytrorum striis levioribus, minus distincte punctulatis interstitiisque magis deplanatis.

23. Cryobius Fatús: oblongus, supra nigro-aeneus, nitidus, subtus niger; thorace deplanato, latitudine breviore, subcordato, posterius sinuato-angustato, angulis rectis, medio canaliculato, postice utrinque evidenter bistriato, impressionibus punctatis; elytris ovatis, striatis, striis subtiliter punctatis, utroque punctis duobus impressis; palpis, antennarum basi, elytrorum margine apicali anguste et epipleuris pedibusque obscure rufis, femoribus infuscatis.

Var. b. elytris aeneo-cuprescentibus.

Var. c. tota rufo-testacea, certe immatura.

Longit. $3 \frac{3}{4}-4$ lin. Latit. $1 \frac{1}{2}-1 \frac{3}{4}$.

Cryobius validus $\left({ }^{*}\right)$ Ménétriés in litteris.

lisdem locis cum praecedente eodemque tempore a D. Holmberg frequentius lectus.

Pterosticho pinguidineo Eschsch. (Mannerh. Bullet. de la Soc. de Moscou. 1843. p. 205. 62.), affinis, sed brevior, thorace breviore, posterius magis subito sinuato - angustato, intra angulos posticos utrinque evidenter bistriato, ibique rugoso-punctato, elytris brevioribus, convexioribus, profundius striatis, striis distinctius punctatis et margine inflexo rufo, femoribusque obscurioribus dignoscendus.

(") Inden dieser Name ciner Art unter den Feronien gegeben worden ist, musste die hier beschriebene einen anderen erhalten. 
24. Cryobius ruficollis: oblongus, subdepressus, piceus; thorace longitudine latitudinis, subcordato, angulis basalibus rectis, postice utringue unistriato, impressionibus impunctatis, basi in medio longitudinaliter subtiliter striguloso, medio canaliculato; elytris oblongo-ovatis, levissime striatis, striis vix punctatis, punctisque tribus impressis; ore, antennarum basi, thorace, corpore subtus pedibusque rufo-ferrugineis, tarsis obscurioribus.

Longit. 3 lin. Latit. $1 \frac{1}{4}$ lin.

In insula Afognak specimen unicum invenit I). Holmberg.

Statura fere omnino Cr. Pinguediner (Pterosticur Mannerh. 1. c.) Eschsch., a quo, praeter magnitudinem, differt praecipue thoracis basi striguloso, elytris levius striatis et colore.

25. Crionius empetricola Eschscholtz.

Ptenostichus empetricola Mannerh. Bullet. de la Soc. de Moscou. 1843. p. 205.63.

Argutor beviconnis? Kirby in Richarlson Fauna Bor. Amer. p. 31. 39. Tab. VIII. fig. 3.

Eliam in ora occidentali insulae Kadjak, in insula Afognal, mense Augusto, et ad sinum Woskresensk peninsulae lienai, mense Majo, sub lapidibus vulgaris; D. llolmberg.

26. Cryorids Fastidiosus: oblongus, supra nigroaeneus, nitidus, subtus nigro-piceus; thorace subcordato, subdepresso, longitudine parum latiore, lateribus rotundato, basi sinuatim subito angustato, angulis rectis, medio canaliculato, postice utrinque profunde unistriato, impressione impunctato; elytris oblongoovatis, disco evidenter striatis, striis obsolete punctu- 
latis, versus latera plus minusve evanescentibus e punctis oblongis seriatis tantum effectis, utroque punctis tribus vel quatuor impressis; antennarum basi pedibusque rufo-piceis.

Longit. $2 \frac{1}{3}-2 \frac{2}{3}$ lin. Latit. $1-1 \frac{1}{6}$ lin.

Uabitat sub lapidibus ad sinum Woskresenk peninsulae Kenai, e medio ad finem Maji, frequens; D. Ilolmberg.

Praecedente minor et ab illo thorace minore, lateribus fere basin usque magis rotundato, elytrorum striis disci profundioribus, quamvis etian e punctis oblongis seriatis saepe confectis, coloreque pedum obscuriore diversus.

27. Criobius rotundicollis Ménétriés : oblongoovatus, supra rufo-castaneus, nitidus, elytris nonnihil aeneo - micantibus, subtus cum pedibus rufo-ferrugineus; fronte olsolete bisulcata; thorace latitudine vix longiore, lateribus angulos posticos usque valde rotundato, angulis his obtusis, medio profunde canaliculato, apice late emarginato, basi utrinque bistriato, ibique impunctato, stria interiore ad medium disci subarcuatim producta; elytris evidenter punctatostriatis, disco punctis tribus minutis impressis; antennis nigro-fuscis, liasi rufo-piceis.

Longit. $4 \frac{1}{4}$ lin. Latit. $1 \frac{3}{4}$ lin.

Habitat in insula Atkha sec. D. Ménétriés, qui individuum ibi captum e Museo Imp. Acad. Scient. Petrop. amice transmisit.

28. Cryorius subaudatus: oblongus, convexus, supra aeneo-cupreus, subtus niger; thorace subcordato, longitudine latiore, postice sinuato - angustato, 
angulis acutiusculis prominulis, basi utrinque unistriato, ad impressiones vix punctulato, medio canaliculato, disco obsolete undulatim strigoso; elytris oblongo-ovatis, convexis, apice sinuato-productis, subcaudatis, leviter punctato - striatis, interstitiis planis punctisque tribus impressis; antennarum articulo primo pedibusque obscure rufis.

Var. b. forsan nuper exclusa, ore, antennarum articulis quatuor baseos, corpore subtus pedibusque pallide ferrugineis.

$$
\text { Longit. } 3 \frac{1}{2}-4 \text { lin. Latit. } 1 \frac{1}{2}-1 \frac{2}{3} \text { lin. }
$$

Ad rivulos fl. Tschunuktnu peninsulae Kenai, sub lapidibus, exitu mensis Junii, a D. F. Frankenhaeuser sat copiose lectus.

29. Cryobius Quabricollis Ménétriés: oblongus, viridi - cupreus, nitidıs; thorace subcordato, longitudine latiore, lateribus antrorsum dilatato - rotundato, medio canaliculato, ante basin apicemque transversim impresso, basi utrinque profunde bistriato, angulis subrectis; elytris oblongis, striatis, striis subtilissime remote punctatis, interstitio tertio utrinque punctis quatuor minutis impressis; antennis pedibusque nigropiceis, illarum articuli primi basi femorumque basi et genubus rufescentibus.

Longit. $1 \frac{2}{3}$ lin. Latit. 1 lin.

Motschulskin, die Käfer Russl. I. p. 55.

Habitat in insula St. Georgii; D. Wosnesensky. Specimen descriptum in IIuseo Imp. Acad. Scient. Petrop. exstat.

Pterosticho snmu Ménétr. (Mannerh. Bullet. de la Soc. de Moscou, 1852. I. p. 296. 9.) vicinus, sed thorace 
subcordato, breviore, latiore, antice posticeque profundius transversim impresso, basi utrinque profundius bistriato, pedibusque obscurioribus diversus.

30. Miscodera Americana: elongata, convexa, supra obscure aenea, nitidissima, subtus nigro-picea, antennis, pedibus anoque rufis; capite thorace angustiore, inter oculos transversim impresso; thorace latitudine sesqui longiore, globoso, pulvinato, basi angustato - constricto, angulis minutis rectis, medio obsoletissime canaliculato; elytris oblongo-ovatis, valde convexis, stria tantum suturali profunde exarata, obsolete punctata.

Longit. $3 \frac{3}{4}$ lin. Latit. $1 \frac{1}{8}$ lin.

Ad fl. Skeljanktnu peninsulae Kenai, medio Junii, specimen unicum sub cortice arboris emortuae invenit D. F. Frankenhaeuser.

M. ancticae Payk. propinqua, sed major, praeterea thorace longiore, basi impunctato elytrisque unistriatis, stria obsolete punctata, praecipue dignoscenda.

31. Leirus carinatus Leconte: subelongatus, nigropiceus, subnitidus, supra interdum nigro-aeneus; thorace lateribus rotundatis, postice modice angustato, non coarctato, antice posticeque crebre punctato, angulis basalibus subrectis, basi utrinque bistriato, carina externa valde distincta; elytris oblongis subparallelis, punctato-striatis; antennis, palpis pedibusque rufo - piceis vel obscure ferrugineis.

Longit. $4 \frac{1}{3}-4 \frac{1}{9}$ lin. Latit. $1 \frac{3}{4}-2$ lin.

Curronotus carinatus Leconte, Catal. of the Geodephagous Coleopt. inhab. the United States (Annals of the Lyceum of Nat. Hist. of New York Vol. IV.) p. 963. 


\section{$43 \quad 135$}

IIabitat sub lapidibus ad ostia fl. Kaktnu peninsulae Kenai, exitu mensis Junii frequens, in insula Kadjak vero rarius; D. Holmberg.

L. tonnipo Panz. valde affinis, thorace latiore, postice non coarclato, basi apiceque crebrius punctato et elytrorum striis subtilius punctatis diversus.

32. Bradytus graciatis Ménétriés: breviusculus, supra cupreo - aeneus, subtus niger, caeruleo-micans, antennis nigris, femoribus obscure rufescentibus; thorace brevissimo, lateribus modice rotundato, postice leviter angustato, angulis rectis, intra illos utrinque bifoveolato, medio canaliculato, antice transversim linea arcuata impresso ibique lateribus et basi punctato; elytris evidenter punctato-striatis.

Var. b. cuprea, pedibus rufis, tibiis posticis basi tarsisque omnibus infuscatis.

Longit. $2 \frac{3}{4}-3 \frac{1}{3}$ lin. Latit. $1 \frac{5}{1} \frac{5}{9}-1 \frac{2}{3}$ lin.

Motschulsky, die Käfer Russl. I. p. 62.

Ad fl. Tchuniten peninsulae Kenai sub lapide individıum unicum, initio Junii, legit D. F. Frankenhacuser. Var. $b$. ut ad litora continentis Americes borealis captam communicavit D. Motschulsky. Occurrit etiam in peninsula Kamschatka.

33. Amara impuncticoleis Say: ovalis, supracupreoaenea, nitida, subconvexa, subtus nigro-picea; thorace antrorsum modice angustato angulis obtusis, apice late emarginato, basi subtruncato angulis fere rectis, medio tenue canaliculato, intra angulos posticos utrinque obsoletissime biimpresso, omnino impunctato; elytris tenue striatis, interstitiis planis aequalibus; antennarum articulis tribus basalibus tibiisque rufoferrugineis. 


\section{4}

Var. b. supra tota nigra, minus nitida, antennarum basi et tibiis ut in $a$.

Longit. 4 lin. Latit. $1 \frac{2}{3}$ lin.

Zimmermann in Gistl Faunus I. p. 35. in Silbermann Revue entom. II. p. 227. - Richardson, Fauna Bor. Amer. IV. p. 39. 53. - Leconte Cat. of the Geodeph. Col. inhab. the Unit. Stat. p. 90. 3.

Feronia impuncticollis Say, Trans. Amer. Phil. Soc. N. S. II. p. 36.3 .

Amara trivialis Var. Dejean, Spec. gén. III p. 46\%. 6. Cat. 3-ême édit. p. 44.

Amara ovalis Sturm, Cat. p. 28.

Amara atripes Ménétrićs in litteris.

Amara tripartita Ménétriés in litteris.

IIabitat in ora orientali insulae Kadjak frequens; D. IIolmberg. Specimina ibidem capta etiam communicavit D. Ménétriés. Individuum paullo majus ex insula Atkha, sub nomine A. tripartitae sibi, a D. Ménétriés ad describendum quoque obtinui.

34. Amara insignis Eschscholt:: Mannerh. Bullet. de la Soc. de Moscou. 1843. p. 208. 68.

Celia compacta Motschulsky, die Kï̈fer Russl. I. p. 59.

IIabitat etiam in insula Sitkha, sec. D. Motschulsky, cujus amicitiae cognitionem individui in Museo ejus sub nomine citato asservati debeo. Dubitare mihi tamen liceat insectum hoc revera insulae Sitkhae incolam esse.

35. Celia erRAtica Duftschmid: ovata, supra cupreoaenea, nitida, laevigata, subtus nigra; thorace antice angustato, basi subtruncato, angulis rectis, medio 
tenue eanaliculato, postice utrinque obsolete bifoveolato impunctato; elytris leviter striatis, striis subtiliter punctulatis; antennis nigris articulo primo interdum rufescente, pedibus nigro-piceis.

Longit. $3-3 \frac{1}{2}$ lin. Latit. $1 \frac{2}{3}-1 \frac{3}{4}$ lin.

Zimmermann in Gistl Faunus I. p. 24. in Silbermann Revue entom. II. p. 213.

Amara erratica Sturm, Fauna VI. p. 55. 31. Tab. CXlVI. fig. b. B. - Bach, Käferfauna I. p. 68. 29. - Gaubil, Cat. p. 34. 16.

Amara (Celia) erratica Cat. Col. Europ. 1852. p. 10.

Amana punctulata (*) Dejean, Spec. gén. III. p. 472.14. Cat. 3-ême édit. p. 44.

Amara septentrionalis Schiölte, Naturhist. Tidskr. I. p. 170.

II arpalus velgaris Zetterstedt, Fauna Ins. Lappon. I. p. 22. 10. Ins. Lappon. p. 35. 11.

Carabus erraticus Duftschmid, Faun. Austr. II. p. 120. 149.

Habitat in ora orientali insulae Kadjak, per totam aestatem frequens; D. Holmberg. In regionibus interioribus et ad ostia fl. Kaktnu peninsulae Kenai a D. F. Frankenhacuser lecta. Specimina in insula Atkha capta, sub nomine Celae punctulatae $D e j$. communicavit D. Ménétriés.

36. Celia indistincta Motschulsky: subovata, supra obscure cupreo-aenea, nitidissima, subtus nigro-picea;

(") Das Citat für diese Art nebst dazu angefertigter Note bei Auna Litтonalis Eschsch. Mannerh. Bullet. de la Soc. de Moscou. 1843. p. 207. 66., als auf einen Irrthum beruhend, müssen also gestrichen werden. 
thorace brevi, longitudine fere duplo latiore, antice subangustato, margine laterali anguste reflexo, basi medio transversim impresso, utrinque profunde bifoveolato, foveis punctatis; elytris levissime striatis, striis remotis, subtilissime remote punctulatis; labri, thoracis elytrorumque margine summo anguste et epipleuris rufescentibus; antennis fuscis, basi rufo-testaceis; pedibus piceis, tibiis tarsisque rufescentibus.

Longit. $3 \frac{3}{4}$ lin. Latit. $1 \frac{5}{6}$ lin.

Motschulsky, die Käfer Russl. I. p. 59.

Iabitat in insula Unalaschka, sec. D. Motschulsky, qui individuum e Museo suo ad describendum mecum benevole communicavit.

amarae (Celiae) reatotestrutae Eschsch. (Mannerh. Bull. de la Soc. de Moscou. 18\%3. p. 208. 69.) valde affinis, sed latior, magis $\mathrm{Cu}^{\mathrm{r}} \mathrm{T}$-nitida, thorace breviore, antrorsum minus ang wivizto, foveolis posticis profundius impressis et punctatio, dasi transversim impresso et. elytrorum striis remotiss punctatis imprimis diversa.

37. Celia relucens: breviter ovata, supra aeneocuprea, lucida, subius nigro-picea; thorace brevi, longitudine duplo laciore, antice subangustato, intra latera circulatim denianato, postice utrinque profunde bifoveolato, circa foveas usque ad medium crebre confertim punctato, medio evidenter canaliculato, basi leviter bisinuato, angulis rectis; elytris striatis, striis vix punctatis; epipleuris, antennis, tibiis tarsisque ferrugineo-testaceis, antennis extrorsum femoribusque infuscatis.

$V a r . b$. ore, thoracis elytrorumque summo margine et corpore subtus cum pedibus pallide ferrugineis.

Longit. $3-3 \frac{3}{4}$ lin. Latít. $1 \frac{3}{2}-1 \frac{2}{3}$ lin. 
In ora orientali insulae Kadjak, per totam aestatem vulgaris et ad ostia 1l. Kaktuu, exitu Junii, sub lignis e mare rejectes et lapidibus sat frequenter obvia; $\mathbf{1 0}$. Holmberg. Etian in regionibus interioribus peninsulae Kenai a D. F. Frankenhaeuser inventa.

Statura fere C. nemotestriatae Eschsch., sed thorace postice crebrius et profundius punctato, foveis binis magis impressis, elytrorum striis evidentioribus, inter se minus distantibus et colore magis aeneo distinguenda.

38. Celia amplicollis: oblongo-ovata, subconvexa, nigro-picea, supra aeneo-micans, nitida; thorace longitudine vix latiore, antrorsum modice angustato, medio canaliculato, basi leviter bisinuato, angulis subrotundatis, postice utrinque leviter bifoveolato, circa foveas profunde punctato; elytris thorace angustioribus et parum longioribus, evidenter striatis, striis impunctatis ; ore, antennis, thoracis summo margine laterali pedibusque rufis.

Longit. $2 \frac{1}{2}$ lin. Latit. 1 lin.

Ad rivulum fl. Tschunuktnu peninsulae Kenai individuum unicum in ligno, initio Julii, cepit D. F. Frankenhaeuser.

C. graxdicoli Dej. Zimmerm. proxima, a qua differt thorace longiore, angustiore, elytrorum striis impunctatis et colore paginae superioris, praesertim elytrorum, aeneomicante.

39. Calatuus ixcomode : oblongus, apterus, subdepressus, nigro-piceus; thorace latitudine longiore, lateribus vix rotundato, intra marginem utrinque longitudinaliter inpresso, margine postice vix reflexo, medio tenuissime canaliculato, basi utrinque obsolete foveolato, angulis rotundatis; elytris oblongo-ovatis, striatis, punctis tribus impressis; palpis, antennis, 


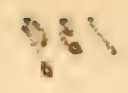

\section{8}

thoracis margine epipleuris pedibusque pallide rufotestaceis.

Var. b. corpore subtus etiam pallide testaceo, capite thoraceque rufescentibus.

$$
\text { Longit. } 3 \frac{1}{4}-4 \text { lin. Latit. } 1 \frac{1}{4}-1 \frac{2}{3} \text { lin. }
$$

Medio aestatis sub lapidibus rarius inventus; a D. Holmberg in insula Kadjak et ad ostia fl. Kaktnu peninsulae Kenai; a D. F. Frankenhaeuser ad rivulos fl. Tschunuktnu ejusdem peninsulae.

C. ingrato Eschsch. (Mannerh. Bullet. de la Soc. de Moscou. 1843. p. 195. 35.) valde affinis, saepius illo minor, magis elongatus, elytris brevioribus et thoracis angulis posticis rotundatis fere unice distinctus.

40. ANCHOMENU LENIS: oblongo-ovatus, nigro-piceus, subdepressus; thorace longitudine vix latiore, versus basin modice lineariter angustato, lateribus posterius explanatis reflexis, angulis basalibus oblique rotundato-subtruncatis, medio tenue canaliculato; elytris oblongo-ovalibus, leviter striatis punctisque duobus impressis; ore, antennis, thoracis limbo anguste pedibusque rufo-piceis.

Longit. $4 \frac{1}{2}-4 \frac{3}{4}$ lin. Latit. $1 \frac{9}{3}-1 \frac{3}{4}$ lin.

Habitat in insula Kadjak ubique frequens; in insula Afognak quoque sub lapidibus mense Augusto haud infrequens; D. Holmberg.

A. moun Eschsch. (Mannerh. Bullet. de la Soc. de Moscou. 1843. p. 198. 43.) nimis affinis, a quo differre tamen videtur thorace longiore angustiore, basin versus magis angustato, ibique lateribus altius reflexo-marginato, angulis subtruncatis et elytris longioribus. 
41. Axchomenus oucrs: subelongatus, nigro-piceus, subdepressus; thorace latitudine longiore, versus basin rotundatim modice angustato, lateribus explanatis posterius parun reflexis, angulis basalibus obtusis subrotundatis, medio profunde canaliculato; elytris oblongo-ovatis, striatis, interstitiis subelevatis punctisque duobus impressis; ore, antennis, thoracis limbo anguste pedibusque rufo-piceis.

Longit. 4 lin. Latit. $1 \frac{1}{9}$ lin.

Habitat in regionibus interioribus peninsulac Kenai; D. F. Frankenhaeuser.

Praecedenti iterum vicinus, statura magis elongata, thorace longiore, angustiore, versus basin haud lineariter sed rotundatim angustato, marginibus minus reflexis, angulis posticis minus oblique truncatis elytrisque brevioribus diversus.

42. Axchomenus Bogemaxi Gyllenhal: oblongus, depressus, aterrimus, nitidus; thorace longitudine latiore, posterius modice angustato, lateribus reflexomarginato, angulis basalibus oblique truncatis, subrotundatis, medio canaliculato, supra subtilissime striguloso, foveis posticis obsoletis; elytris elongatis, parallelis, interstitiis alternis latioribus, punctisque obsoletis tribus impressis.

Longit. $3 \frac{1}{2}$ lin. Latit. $1 \frac{1}{2}$ lin.

Gaulil, Cat. p. 28. 53.

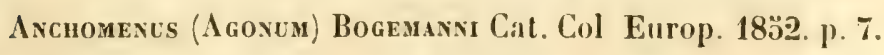
Agonum Bogemaxai Dejean, Spec gén III p. 171. in. Iconogr. II. p. 385. 30. Tab. 122. fig. 6. Cat. 3-ème édit. p. 36. 


\section{the 50}

Harpalus Bogemanni Gyllenhal, Ins. Suec. III. p. 697. 57-58. IV. p. 448. 57-58. - Sahlberg, Ins. Fenn. I. p. 253.63.

Mense Augusto excipulo e gramine ad rivulum fl. Tsehunuktnu peninsulae Kenai specimen unicum cepit D. F. Frankenhaeuser.

A specie Boreali-Americana nomine Agon tuctuosi $D e j$. cognita, in museo meo etiam exstante, satis distinctus.

43. Aлchomenus gratiosus: subelongatus, supra nigro - aeneus, subtus nigro - piceus; thorace latitudine longiore, apice profunde emarginato, lateribus modice rotundato, margine praesertim postice reflexo, basi utrinque longitudinaliter impresso, angulis rotundatis, medio canaliculato; elytris ovalibus, simpliciter striatis, interstitio tertio punctis quatuor impressis; antennis extrorsum pedibusque testaceo - ferrugineis.

Longit. $3 \frac{1}{2}$ lin. Latit. $1 \frac{1}{2}$ lin.

Habitat in insula Kadjak sub lapidibus rarius; D. Holmberg.

Magnitudine $\Lambda$ Gonı monosı $D e j$, a quo thorace multo longiore et colore satis diversus.

44. Anchomenus Fragilis: elongatus, niger, nitidus, subaeneo - micans; thorace oblongo-quadrato, elytrorum latitudine duplo angustiore, lateribus vix rotundato, medio canaliculato, basi utrinque oblique truncato, angulis obtusis, ibique margine valde reflexo, fovea longitudinali utrinque impressa; elytris elongatis, subparallelis, basi conjunctim valde emarginatis, subtiliter striatis, punctisque quinque impressis.

Longit. 3 lin. Latit. 1t lin. 
Habitat in insula Sitkha rarissime; D. Holmberg.

Agoxo gracru Sturm, Dej. fere ut ovum ovo simillimus, sed accuratiori examini subjectus speciem revera diversam constituere videtur; thorax nempe ratione elytrorum minor, magis quadratus, margine postico magis reflexo, elytra longiora, lateribus parallela, ita ut tantum paullo ante apicem rotundato-angustata, et color nonnihil aeneo-micans.

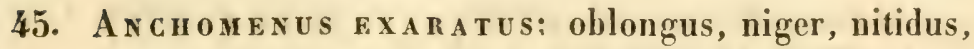
thorace elytrisque cupreo-aeneis; illo longitudine breviore, elytris duplo angustiore, subquadrato vel posterius parum angustato, basi utrinque oblique truncato, angulis obtusis, medio canaliculato, antice linea arcuata impresso, postice utrinque fovea oblonga profunde excavato, margine laterali explanato - reflexo; elytris subovatis, striatis, striis praesertim in disco profunde exaratis, utroque punctis quatuor minutis impressis.

Longit. 3 lin. Latit. $1 \frac{1}{3}$ lin.

In ora orientali insulae Kadjak, mense Angusto, sub Iapidibus a D. Holmberg inventus.

46. Anсu OMENUS Bembidoides Kirby: oblongus, depressus, supra nigro-aeneus, opacus, subtus niger, nitidus; thorace latitudine breviore, elytris fere duplo angustiore, basi apiceque subtruncato, angulis obtusis, lateribus rotundatis, reflexo-marginatis, subtilissime confertim aciculato, medio profunde canaliculato, utrinque in disco paullo ante medium foveola impresso, ad angulos posticos leviter foveolato; elytris oblongo - quadratis, humeris rotundato - productis, apice sinuato - truncatis, undique leviter interrupto- 


\section{ใด เ 52}

striatis, aeneo-sericatis, maculis numerosis, introrsum majoribus difformibus atro-holosericeis, utrinque in interstitio tertio serie e foveis quatuor majoribus parum excavatis, sub - argentatis.

Longit. 3 lin. Latit. 1 lin.

Sericoda Bembidordes Kirby in Richardson Fauna Bor. Amer. IV. p. 15. 11. Tab. I. fig. 2.

Agonum Bembinromes Leconte, Cat. of the Geodeph. Col. inhab. the Unit. Stat. p 55. 19.

Penrphus cicatricosos Ménétriés, Motschulsky die Käfer Russl. I. p. 11.

Ad rivulos fl. Tschunuktnu peninsulae Kenai, initio Septembris, in volatu cepit D. F. Frankenhaeuser. Individua duo ad fl. Kiwichpakh a D. Sagosskin lecta e Museo Acad. Imp. Scientiar. Petrop. ad describendum etiam obtinui.

47. Ancuomenus octocolus Chaudoir: oblongus, depressus, nigro-aeneus; thorace transversim quadrato, elytris parum angustiore, medio canaliculato, lateribus modice rotundatis, late explanatis, margine vix reflexo, postice utrinque oblique truncato, angulis valde obtusis, foveola basali nulla, in disco anteriore utrinque puncto impresso; elytris obsolete striatis, striis hinc inde interruptis, utroque foveis majoribus quatuor impressis.

Longit. 2 lin. Latit. 1 lin.

Agonothorax octocolus Motschulsky, die Käfer Russl. p. 69.

Ad rivulos fl. Tschunuktnu peninsulae Kenai, initio Septembris, in volatu a D. F. Frankenhaeuser rarius captus. Specimen e museo suo etiam amice communicavit D. Motschulsky. 
Agonum quadrinumctatum $D e$ Geer, $D e j$. facillime mentiens, sed brevior, praesertim in elytris, capite thoraceque latioribus, hoc magis quadrato, lateribus latius explanato, impressionibus basalibus nullis, elytris obsoletius et interrupte striatis, foveis impressis majoribus dignoscendus.

In individuo altero e duobus a D. Frankenhaeuser captis, elytron dextrum foveis sex notatum, quarum duae in serie altera mox pone medium positae; in specimine vero a D. Motschulsliy communicato foveolae in medio extus utrinque alia minuta aggregata; sed aberrationes haec ut lusus naturae considerandae.

48. Patrobus Lorgiventris: apterus, nigro-piceus, subdepressus; capite thorace parum angustiore, fronte profunde liimpressa, transversim rugosa; thorace longitudine vix latiore, modice cordato, medio canaliculato, basi utrinque late et profunde foveolato, foveis rugoso-punctatis, bicarinatis; elytris thorace triplo longioribus et ejus latitudine posterius duplo latioribus, punctato - striatis, utroque punctis tribus impressis; palpis, antennarum articulo primo pedibusque piceis.

Longit. $5 \frac{1}{2}$ lin. Latit. $2 \frac{1}{5}$ lin.

Habitat in ora orientali insulae Kadjak, mensibus Julio et Augusto, sub lapidibus frequens; D. Holmberg.

P. Fossifronte Eschsch. (Mannerh. Bullet. de la Soc. de Moscou. 1843. p. 194. 33.) major, praeterea thorace angustiore, lateribus minus rotundato, foveis bicarinatis elytrisque longioribus et latioribus diversus.

49. Patrobus Fulves: apterus, subdepressus, fulvoferrugineus, capite thoraceque rufescentibus; hoc longitudine plus quam sesqui latiore, subcordato, 
medio canaliculato, angulis posticis oblique subtruncalis, basi utrinque late foveolato, foveis rugosopunctatis, bistriatis; elytris oblongo-ovatis, subtiliter punctato-striatis, utroque punctis tribus impressis.

Longit. 5 lin. Latit. $1 \frac{5}{6}$ lin.

Ifabitat in insula Karljak rarissime; 1). Holmberg.

A P. rossrfnoxte Eschesch. differt colore, statura magis depressa, thorace capite muito latiore, foveis basalibus minus profundis bistriatis elytrisque ratione thoracis majoribus.

50. Patrobus axgetsticollis: elongatus, apterus, nigro - piceus, antennis, pedibus abdomineque rufis; fronte obsolete biimpressa; thorace latitudine fere longiore, lateribus vix rotundato, posterius parum angustato, medio canaliculato, basi utrinque late foveolato, foveis remote punctatis, extus bistriatis; elytris thorace parums latioribus, oblongo-ovalibus, strialis, striis antrorsum punctatis, utroque punctis tribus minutis impressis.

Longit. $4 \frac{1}{5}$ lin. Latit. $1 \frac{1}{3}$ lin.

Al sinum Woskresensk peninsulae Kenai a D. Ifolmberg inventus.

P. L..custri Motsch. (Ins. de la Sibérie. p. 130. 199.) qunodammodo similis, sed magis elongatus, fronte obsolete bifoveolata, thorace longiore, lateribus minus rotundato, foveis posticis minus profundis elytrisque longioribus et abdomine rufo-discrepans.

51. Tacinpus elongates Motschulsky: elongatus, supra fusco-aeneus, sub(iliter crebre punctulatus, tenue griseo-pubescens, subtus nigro-piceus; capite thorace latiore, oculis magnis prominulis; thorace 
latitudine longiore, cordato, medio canaliculato, laleribus antrorsum modice rotundato; elytris oblongis, lateribus subparallelis, griseo-tessellatis punclisque duobus impressis; antennis fuscis, articulo primo nigro-aeneo, pedibus ferrugineo - testaceis, femoribus apice infuscatis.

Longit. $2 \frac{3}{4}$ lin. Latit. 1 lin.

Motschulskiy, die Käfer Russl. I. p. 16.

IJabitat in insula Sitkha, sec. I). Molschulstiy, sed signo interrogationis ut hujus loci incola in opere citato inscriptus, quare exinde allatum fuisse eo vehementius dubitarem. Typum ad deseribendum mihi benevole transmisit D. Molschulsky.

T. PICIPEDE Megerle, Dej. longior, angustior, capite thorace latiore, hoc lateribus antrorsum minus dilatato, elytris Iongioribus, angustioribus, lateribus subparallelis, maculis griseis tessellatis magis ordinatis, colore fuseoaeneo, minus cupreo pedibusque pallidioribus distinguendus.

52. Bembidum GLariusculua Motschulsky: breviter ovatum, subdepressum, nigrum, nitidum; capite utrinque profunde sulcato; thorace transverso, longitudine fere duplo latiore, lateribus parum rotundatis, angulis anticis nonnihil rotundato-productis, postice vix sinuato-angustato, per totam longitudinem in medio canaliculato, basi transversim impresso, punctulato, ibique in medio late producto, utrinque oblique truncato, angulis obtusis, intra angulos foveolatim impresso; elytris punctato-striatis, interstitio tertio punctis duobus impressis; antennarum articulo primo tibiisque rufis.

Longit. 3 lin. Latit. $1 \frac{1}{3}$ lin. 
Motschulsky, die Käfer Russl. I. p. 15.

Habitat in insula Sitkha, sec. D. Motschulsky, qui individuum in museo ejus asservatum mecum amice communicavit.

53. Notaphus unduatus Sturm : oblongus, supra fusco-aeneus; fronte profunde bisulcata; thorace longitudine fere sesqui latiore, subcordato, basi utrinque bistriato, angulis posticis rectis; elytris oblongoovatis, profunde striatis, striis antrorsum profunde, postice vero leviter punctatis, fasciis transversis undatis macularibus tribus, quarum prima et secunda confusis, apiceque rufo - testaceis, punctisque duobus impressis; antennarum basi pedibusque rufo-testaceis, femoribus nonnihil infuscatis.

Longit. $2 \frac{1}{4}$ lin. Latit. 1 lin.

Motschulsky, die Käfer Russl. I. p. 15.

Bemidium undulatum Sturm, Faun VI. p 156. 33. Tab. CLX. fig. d. D. - Sahlberg, Ins. Fenn. I. p. 202. 27. Dejean, Spec. gén. V.p 63. 27. Iconogr IV. p. 343. 18. Tab. 209. fig. 6. Cat. 3-ème édit. p. 57. - Gaubil, Cat. p. 41. 23. - Bach, Käferfauna. I. p. 85. 17.

Bembidium masus Gyllenhal, Ins. Suec. IV. p. 411. 15-16.

Ad ostia fl. Kaktnu peninsulae Kenai a D. Holmberg semel captus.

Specimen unicum inventum elytris basi paullo fortius quam in individuis Europaeis punctato-striatis discrepans, sed ceterum is omni puncto simillimum.

54. Notapir Q Q A D A T I C L Is: breviusculus, subdepressus, supra nigro-aeneus, nonnihil virescens, subopacus, subtus cum antennis pedibusque niger; fronte 


\section{$57 \quad 149$}

profunde bisulcata; thorace longitudine sesqui latiore, antice posticeque latitudine aequali, lateribus modice rotundato, basi haud coarctato, angulis rectis, in medio tenue canaliculato, lateribus et basi punctulato, postice utrinque bistriato; elytris thorace fere duplo latioribus, totis evidenter punctato-striatis, fasciis duabus transversis macularibus, una ante, altera pone medium, testaceo-pallidis.

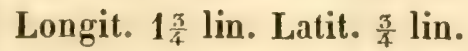

Habitat ad ostia fl. Kaktnu peninsulae Kenai rarissime; D. Holmberg.

N. овцден Sturm, Dej. vicinus, sed thorace minore, fere quadrato, postice haud coarctato elytrorumque striis undique aequaliter exaratis ab illo mox dignoscendus.

55. Perypues bimaculatus Kirby: supra obscure viride-aeneus, subtus nigro-piceus, nitidus; capite profunde bifulcato; thorace obcordato, antice emarginato, basin versus valde angustato, angulis rectis, dorso convexo, medio canaliculato, basi fortiter punctato ibique utrinque profunde foveolato; elytris leviter punctato - striatis, striis externis posterius evanescentibus, disco punctis impressis duobus minutis utrinque, intra apicem utroque macula oblonga, testaceo lurida; palpis, antennarum articulo primo, cingulo in duobus insequentibus, trochanteribus, femorum basi, tibiis tarsisque rufo-ferrugineis.

Longit. $2 \frac{3}{4}$ lin. Latit. 1 lin.

Richardson, Fauna Bor. Amer. IV. p. 52. 74. - Leconte, Cat. of the Geodeph. Col. inhab. the Un. Stat. p. 194. 36.

Ad castellum Nicolajevsk sinus Kenai vel Cooks inlet a D.

Holmberg semel captus. 
P. infuscito Dej. valde affinis, sed oculis minus globosis, capite profundius bisulcato, thorace paullo latiore, minus convexo et elytris nonnihil longioribus diversus.

56. Perypus úcrous (*) Leconte: elongatus, capite thoraceque viridi-aencis splendidis; fronte profunde bisulcata; thorace latitudine plus sesqui breviore, lateribus rotundatis, postice breviter sinuatis, angulis subrectis, basi subtiliter punctato ibique trausversim impresso, utrinque profunde foveolato; elytris oblongis, tenue punctato - striatis, interstitiis planis, fusco piceis, virescentibus, utroque macula humerali oblonga alteraque obliqua ante apicem flavo - testaceis; antemarum basi, epipleuris pedibusque rufis, femoribus nomnihil infuscatis.

Var. b. elytris pallide flavo-testaceis, umbra suturali pone sculellum maculaque media communi cum subscutellari sacpe cohaerente, interdum antrorsum biloba, et ante apicem infuscalis.

Longit. $2 \frac{1}{4}-2 \frac{3}{4}$ lin. Latit. $\frac{2}{3}-1$ lin.

Leconte, Cat. of the Geodeph. Col. of the Unit. Stat. p. 19\%. 34.

Ilabitat in arena litorali maris ad castellum Nicolajevsk peninsulae Kenai, mense Junio, sat frequens; D. Holmbery.

P. Axdreae Fabr. (mupestri Illig. Dcj.) colore subsimilis, sed magis praesertim elytris elongatus, deplanatus, thorace posterius minus coarctato el elytris levius striatis, in striis subtiliter punctatis.

(*) Der Name Lucnous wurde wohl friiher an eine Art derselben Gattung von Faldermann (Fauna Entom. Transcauc. I. p. 202.) vergeben; allein da Herr von Motschulsky (die Käfer Russl. p. 9.) bewiesen hat, dass diese nichts anderes als P. nufiprs Rossi, Dej. sei, habe ich keinen Anstand genommen den fraglichen Namen hiex beizubehalten. 
57. Perroues picipes hirby: niger, nitidus; capite profunde bisulcato; thorace obcordato, longitudine paullo latiore, posterius modice angustato, in medio profunde canaliculato, basi utringue fovea magna impressa in fundo punctata, bistriata, angulis rectis; elytris striatis, striis antice punctatis, versus latera evanescentibus; ore, antennarum articulo primo pedibusque piceis.

Longit. $2 \frac{1}{4}$ lin. Latit. $\frac{3}{4}$ lin.

Richardson, Fauna Bor. Amer. IV. p. J's. 78. - Leconle, Cat. of the Geodeph. Col. inhab. the Unit. Stat. p. 193.31.

Habitat in ora orientali insulae Kadjak, mensibus Julio et Augusto, sub lapidibus rarius; D. Holmberg. Etiam in regionibus interioribus peninsulae Lenai a D. F. Frankenhaeuser inventus.

P. Sumlbengr Dej. nonnihil similis, sed major, latior, pracesertim in thorace, elytris longioribus, levius punctatostriatis et colore obscuriore distinctus.

58. Peryphos tetraglytus: breviusculus, depressus, supra nigro-aeneus, niticlus, subtus cum antennis pedibusque niger; fronte profunde bisulcata; thorace elytris vix angustiore, longitudine fere duplo latiore, basi vix angustato, angulis rectis, utrinque intra angulos crebre punctato, bistriato, dorso canaliculato; elytris sublaevigatis, striis fere omnino evanescentibus, utroque foveis duabus profunde impressis.

Longit. 2 lin. Latit. $\frac{5}{6}$ lin.

In insula Kadjak et ad sinum Nutschek insulae Chtagaluk sub lapidibus locis humidis a D. Holmberg rarius lectus.

Benimio ixcerto Molsch. (Mannerh. Bullet. de Moscou. 18522. I. p. 301. 20.) affinis, sed latior, praesertim thorare, 
magis laevigatus, elytris fere omnino laevigatis, striis vix conspicuis diversus.

59. Perypue complandus : breviusculus, depressus, supra nigro-aeneus, nitidissimus, subtus cum antennis pedibusque niger; fronte profunde bisulcata; thorace Iongitudine duplo latiore, posterius parum angustato, angulis subobtusis, medio canaliculato, basi punctulato, utrinque bistriato; elytris thorace parum latioribus, breviter ovatis, striatis, interstitiis subconvexis, tertio punctis duobus minutis impresso.

Longit. $1 \frac{2}{3}$ lin. Latit. $\frac{2}{3}$ lin.

Habitat in ora orientali insulae Kadjak, mensibus Julio et Augusto, sub lapidibus rarius; D. Holmberg.

Statura depressa Bembidi planiuscum Mannerh, (Bullet. de la Soc. de Moscou. 1843. p. 216. 87.) sed minor, brevior, magis nitidus, thorace latiore, posterius parum angustato, elytris postice magis angustato-rotundatis punctisque illorum impressis minus conspicuis distinctus.

60. TACIYS RIVULA R IS Motschulsky: depressiusculus, nigro-piceus; thorace transverso, posterius modice angustato, angulis basalibus subobtusis, fovea utrinque impressa, medio canaliculato; elytris obscure castaneis, margine et disco suturali obsolete caerulescenti - micantibus, utroque quadristriato, stria suturali integra, ceteris postice evanescentibus, exterioribus gradatim brevioribus.

Longit. $1 \frac{1}{3}$ lin. Latit. $\frac{7}{12}$ lin.

Motschulsky, die Käfer Russl. I. p. 8.

Habitat in insula Sitkha, sec. D. Motschulsky, cujus bene volentiae cognitionem speciminis deseripti debeo. 


\section{Y T I S C I A.}

61. Acilius abireviatus Eschscholtz: Mannerh. Bull. de la Soc. de Moscou. 1843. p. 219. 92.

Motschulsky, Hydrocanthares de la Russie, Cat. p. 9.

Habitat etiam in insula Kadjak; D. Holmberg - Individuum in insula Atkha inventum quoque communicavit D. Ménétriés.

62. Dxtiscus Ooligurin Kirby: Mannerh. Bullet. de la Soc. de Moscou. 1852. I. p. 203. 24. Mas.

Longit. $13-14 \frac{1}{2}$ lin. Latit. $6 \frac{1}{4}-8 \frac{1}{4}$ lin.

F emina: capite subtiliter remotius, thorace subtiliter creberrime punctatis; elytris ultra medium profunde sulcatis, sulcis in fundo luteis, apice crebrius et profundius quam in mare punctis et foveolis confuse impressis.

Longit. $13-13 \frac{1}{2}$ lin. Latit. $6 \frac{3}{4}-7$ lin.

Var. b. capite olivaceo-virescente, thorace elytrisque nigro-piceis: mas et femina.

In lacusculo insulae Kadjak, prope portum Pauli, initio Septembris, copiose legit D. Holmberg. Specimina ex insulis Unalaschka, Sitkha et Kadjak allata, in Museo Acad. Imp. Scient. Petropol. asservata, etiam communicavit D. Ménétriés.

D. marginal Linné angustior, elytris apice crebrius punctulatis et foveolis numerosis confuse impressis, feminae longe ultra medium sulcatis, pagina corporis inferiore flavo-testacea, suturis et segmentorum marginibus nigroinfuscatis ab illo satis diversus. D. anxius Mannerh. (Bullet. de la Soc. de Moscou. 1843. p. 218. 91.) speciei jam descriptae quoque affinis, sed magis laevigatus et 
nitidus, disperse punctulatus, punctura versus apicen elytrorum, quamvis in femina, cujus elytra etiam laevia, nomnihil condensata, foveolis majoribus haud intermixta et corpore subtus in mare suturis et segmentorum marginibus angustius infuseatis, in femina fere omnino pallide flavo-testaceo pedibusque pallidioribus discrepans.

63. Dytiscus parvus Ménétriés: ovalis, brevior, in elytris vix dilatatus, supra olivaceo-niger, nitidus, subtus flavo-testaceus, suturis et segmentorum marginibus anguste nigris; thoracis limbo omni late, elytrorum lateribus et fascia angusta antrorsum curvata, longe intra apicem, interdum obsoleta, flavis; thorace medio tenue canaliculato; elytris in disco disperse, versus apicem crebrius punctulatis; metasterni lobis acuminatis; tarsis posticis nigro-piceis. (Mas.)

Femina: capite thoraceque distinctius subtiliter punctulatis; elytris laevibus, versus apicem crebrius quam in mare punctatis.

Longit. $11 \frac{1}{5}-12$ lin. Latit. 6 lin.

Motschulsky, Etudes entomologiques 1852. p. 77.

Iisdem locis insulae Kadjak ut praecedens species a 1). ILolmberg frequenter captus; individua in eadem insula lecta etiam transmisit D. Ménétriés.

Praccedente minor, brevior, elytris in utroque sexu laevibus, remolius punculatis, apice foveolatis ef fascia postica transversa magis ab apice remota imprimis dignoscendus.

64. Cran a topterus obscuratus Molschulsky: oblongoovatus, ultra medium valde ampliatus, posterius modice attenuatus; supra nigro-fuscus, subtus cum 
pedibus aterrimus; labro, frontis margine antico late bisinuato, maculis binis obsoletissimis in medio, antennarum basi thoracis elytrorumgue limbo omni anguste rufo-testaceis; elytris transversim subtilissime strigulosis.

Var. b. thoracis limbo late ferrugineo-testaceo, vel thorace ferrugineo-testaceo cum fascia transversa nigra; elytris in fundo obscure testaceis, lineolis undulatis transversis nigris subtilissime strigulosis, margine basali et laterali dilutioribus.

Longit. $8 \frac{1}{2}$ lin. Latit. $4 \frac{1}{2}$ lin.

IIabitat in aquis insulae Kadjak rarius. Typum mihi benevole communicavit I). Motschulskiy; Var. b. a I. IIolmberg capta.

Statura ultra medium ampliata Colrubers Dumunac Mannerh. Aubé, sed elytra postice minus attenuata et color obscurior, ceterum ab illo et a Colrmbete Pavkulin Erichs. (sтnisto Gyllenh. Fusco Aubé) striolis transversis elytrorum multo subtilioribus et densioribus abunde distinctus.

65. Crantoptercs dolabratus Paykull: oblongoovalis, niger, capite antice, macula inter oculos didyma saepe cum margine antico confluente, thorace, elytris, segmentorum trium ventralium marginibus, ano, anteunis pedibusque rufo - testaceis; antemnis extrorsum, femorum macula oblonga tarsisque poslicis infuscatis; thorace subtilissime crebre punctulato, lascia, interdum macula, media transversa nigra; elytris lineolis transversim undulatis impressis nigris mediocriter strigosis, e quibus colore fusco - brunneo, margine basali, laterali et epipleuris pallide ferrugineis; 
utroque seriebus quatuor e punctis minutis impressis, suturali prima regulari simplici, reliquis duplicatis posticeque macula minuta nigrescente.

Longit. $6 \frac{3}{4}-7 \frac{1}{3}$ lin. Latit. $3 \frac{1}{3}-3 \frac{2}{5}$ lin.

Dejean, Cat 3-ême édit. p. 61. - Motschulsky, Hydrocan thares de la Russie, Cat. p. 7.

Colymbetes dolabratus Erichson, Gen. Dyt. p. 33. - Aubé, Spec. gén. p. 232. 10. Iconographie. V. p. 103. 7. tab. 13. fig. 2. - Gaubil, Cat. p. 44. 6. - Cat. Col. Europ. 1852. p. 14. Dxtiscus dolabratus Paykull, Fauna Suec. I. p. 204. 13. - Gyllenhal, Ins. Suec. I. p. 478. 12. - Sallberg, Ins. Fenn. I. p. 158. 12. - Zetterstedt, Fauna Lappon. I. p. 210. 9. Ins. Lappon. p. 129. 11.

In aquis stagnantibus insulae Kadjak semel invenit $\mathbf{D}$. Holmberg; individuum alterum ad sinum Woskresensk peninsulae Kenai, exitu M:iji, a D. F. Frankenhaeuser captum.

66. Ilybius picipes Kirby: oblongo-ellipticus, convexus; supra aeneo - niger obscurus, reticulatim confertissime acuductus, subtus aterrimus; vertice vix conspicue rufo-bimaculato; thorace antice serie duplici e punctis minutis marginato, margine basali punctis vix conspicuis, ante scutellum omnino nullis; elytris punctis minutissimis obsolete impressis rarioribus, hinc inde sparsis, vix in series digestis; labro, antennis pedibusque quatuor anterioribus piceis.

Longit. $4 \frac{1}{4}$ lin. Latit. 2 lin.

Colxmbetes picipes Richardson, Fauna Bor. Amer. IV.p 71. 105.

Habitat in aquis ad sinum Woskresensk peninsulae Kenai rarissime; D. Holmberg. 
I.. SUbaeneo Erichs, valde affinis, thoracis seriebus punctatis et punctis impressis elytrorum minus conspicuis, verticis maculis rufis minus distinguendis, ut et antennis pedibusque obscurioribus fere unice distinctus.

67. A gавиs suворастs Motschulsly: oblongo-ovalis, depressus, niger, subtilissime reticulato-strigosus; palpis et antennis basi, fronte antice triangulariter, vertice lineolis duabus transversis, thorace antice et lateribus late, epipleuris pedibusque quatuor anterioribus rufo-ferrugineis; elytris obsolete subsulcatis, obscure ferrugineo-testaceis, confertim et crebre nigro-irroratis, maculis sparsis nigris majoribus.

Longit. 5 lin. Latit. $2 \frac{\pi}{4}$ lin.

Habitat in insula Kadjak, sec. D. Motschulsky, qui individuum in museo ejus exstans ad describendum milhi amice transmisit.

Magnus et insignis in hoc genere, statura A. Arcticı Payk., notis autem pluribus a congeneribus abunde diversus.

68. Agabus atratus: oblongo-ovalis, aterrimus, opacus, striis anastomozantibus subtilissime reticulatostrigosus; ore, antennis, capite antice, epipleuris, segmentorum ventralium marginibus, tibiis tarsisque quatuor anterioribus obscure rufis; elytrorum lateribus disco parum dilutioribus; pedibus posticis piceis.

Longit. 5 lin. Latit. $2 \frac{2}{3}$ lin.

Ad ostia fl. Kaktnu peninsulae Kenai a D. Holmberg semel captus.

A. вipustolato Linné affinis, sed latior et sculptura multo subtiliore, haud longitudinaliter strigosa, sed reticulata ab illo dissimilis. 
69. Agabus semipurctatus Kirby: ovalis; modice convexus, niger, nitidus, supra subaeneo-micans, subtilissime reticulato-strigulosus; vertice maculis duabus rufis; thorace antice serie punctorum contigua aliaque ad basin in medio late interrupta, in ipso medio strigula longitudinali impressa vix conspicua; elytris seriebus tribus e punctis impressis, suturali punctis distantibus regularibus, discoidalibus vero sine ordine congestis, lateribus disperse punctatis dilutioribus, pone medium posterius prope marginem utrinque lineola rufescenti, interdum obsoleta; ore, antennis tarsisque quatuor anterioribus et sacpe tibiis anticis rufo-ferrugineis.

Longit. $3 \frac{3}{4}$ lin. Latit. 2 lin.

Colymbetes semipunctates, Richardson Fanna Bor. Amer. IV. p. 69. 100.

Habitat in stagnis ad sinum Woskresensk peninsulac Kenai, exitu Maji, rarius; D. F. Franlienhacuser.

70. Agabus bicolor Kirby: ellipticus, convexus, niger, nitidus, subtiliter reticulato-strigulosus; vertice maculis duabus obscure rufis obsoletis; thorace seriebus punctorum apicali et basali integris, in ipso medio strigula longitudinali impressa vix conspicua; elytris utrinque in disco serie longitudinali e punctis subgeminatis et praeterea punctis impressis fere undique sine ordine sparsis, margine humerali et externo ultra medium pedibusque rufo-brunneis; ore, antennisque testaceis.

Longit. $3 \frac{1}{4}$ lin. Latit. $1 \frac{1}{2}$ lin.

Coly mbetes bicolor, Richardson Fauna Bor. Amer. IV. p. 70. 101. 


\section{7}

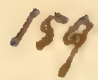

Ad sinum Woskresensk peninsulae Kenai a D. Holmbery rarissime inventus.

71. Agabus praeopterde Kirby: ovalis, subdepressus, niger, nitidus, subtilissime reticulato-strigulosus; vertice maculis duabus obscure rufis; thorace seriebus apicali et basali dense punctatis, hac in medio late interrupta; elytris fusco-castaneis, margine basali et laterali dilutioribus, seriebus tribus longitudinalibus saepe obsoletis e punctis minus regulariter dispositis et insuper punctis aliis versus latera sparsis; ore, antennis, thoracis margine laterali angustissime pedibusque rufo-ferrugineis, femoribus nonnihil infuscatis.

Longit. $3-3 \frac{1}{2}$ lin. Latit. $1 \frac{3}{4}-2$ lin.

Colvmbes phaeoptenus, Richardson Fauna. Bor. Amer. IV. p. 70.102.

IIabitat in insula $\Lambda$ fognak et ad sinum Woskresensk peninsulae Kenai rarissime; D. Holmberg.

A. paludoso Fabr. valde similis et affinis, sed paullo longior, fronte tota nigra et thorace subtilius punctato angustiusque rufo-marginato diversus.

72. AgABUS IR REULARis: ovatus, minus convexus, posterius rotundatus, nigro-piceus, supra nomnihil acneo-micans, nitidissimus, subtilissime punctulatus; ore, antennis, elytrorumque margine late, rufo-ferrugineis; thorace impressionibus aliquot inaequali; elytris punctis numerosis majoribus impressis irregulariter subseriatis; pedibus piceis tibiis tarsisque auterioribus parum dilutioribus.

Longit. 3 lin. Latit. $1 \frac{\mathrm{e}}{3} \mathrm{lin}$. 
Ad rivulum fl. Tschunuktnu peninsulae Kenai, medio Septembris, individuum unicum in volatu cepit D. F. Frankenhaeuser.

A. femorali Payle similis, sed posterius minus angustatus, thorace toto nigro, inaequali, punctura multo subtiliore et elytrorum punctis subseriatis majoribus magis numerosis et irregulariter congestis ab illo dignoscendus.

73. Laccopinus trungatus Ménétriés: ovalis, depressiusculus, luteo-testaceus, posterius attenuatus, apice oblique subtruncatus; thorace postice in medio breviter acute producto; elytris pellucidis, minus crebre nigro - irroratis, maculis irregularibus albidoornatis nigro-circumcinctis, quarum tres in basi contiguae, una major prope illas juxta suturam antice posticeque emarginata, duae minutae mox pone basin cuneatae, una fere in medio ad suturam sublinearis, una marginalis lata a basi ad medium producta, intus lacerata et postice dilatata, una ninuta marginalis prope apicem hamata et ultima apicem occupans dilatata ad suturam ascendens.

Longit. $2 \frac{1}{3}$ lin. Latit. $1 \frac{1}{3}$ lin.

Motschulsky, Hydrocanth. de la Russie p. 9.

Habitat in peninsula Kenai et in insula St. Georgii, sec. D. Ménétriés, qui specimina in Museo Acad. Imp. Scient. Petrop. asservata ad describendum milhi benevole tradidit.

L. Maculoso Knoch, Say, $A u b e$ valde affinis, a quo praecipue differt elytris posterius magis attenuatis, pallidioribus, minus crebre nigro-irroratis, maculis albidis, laterali magis dilatata et postica marginali hamata ab hac magis distante versus apicalem propius remola. 
4\%. HYDROPORUS DECEMLINEATUS： Oblongo - ovalis, niger, nitidulus; fronte triangulariter flavo - testaceo: thorace longitudine triplo latiore, antice valde angustato, crebre punctulato, flavo-testaceo, in medio baseos transversim nigro-fusco; elytris crebrius et paullo profundius punctatis, testaceis, disco infuscatis, simul sumtis apice rotundatis, striis quatuor e punctis minoribus antice exaratis et lineis quinque practer suturam utrinque nigro-ornatis, postice obsoletis, quarum externa antice abbreviata; antennis pedibusque testaceo-ferrugineis.

Longit. $2 \frac{1}{4}$ lin. Latit. 1 lin.

Habitat in stagnis prope castellum Nicolajevsk peninsulae. Kenai, exitu Junii, non infrequens; D. Ilolmberg.

II. picipedi Fabr. valde affinis, colore pallidiore, thorace paullo longiore, antrorsum magis angustato et punctura totius paginae superioris minus profunda et magis condensata $a b$ illo distinguendus.

75. Hydoporus Signatus: oblongo-ovalis, depressiusculus, subtilissime punctulatus, niger, dense et longe griseo-pubescens, vix nitidus; capite antice posticeque, antennarum basi pedibusque rufo-ferrugineis; thorace latissimo, transverso, lateribus oblique subrotundatis, angustissime rufis; elytris pone medium angustato-rotundatis, basi fascia dentala cum macula marginali, ante medium rarum curvatum intus cmittente, cohaerente, maculisque minutis, una oblonga pone medium prope marginem, altera subapicali, saepius obsoletis, rufo-ferrugineis.

Longit. $1 \frac{1}{2}-1 \frac{2}{3}$ lin. Latit. $\frac{3}{4}-1$ lin.

Habitat in aquis stagnantibus insulae Kadjak rarius.

D. Holmberg. 
II. Palustri Linné, Erichs. (sexpustulato Fabr. Gyllenh. $\left.A u b e^{\prime}\right)$ major, latior, thorace multo latiore, elytris pone medium tantum angustatis et colore praeterea ab illo satis diversus:

76. Hydroporus truxcates: elongato-ovalis, subellipticus, depressiusculus, subtilissime et creberrime reticulato - strigulosus, tenue griseo-pubescens; capite rufo-ferrugineo, in fronte infuscato; thorace antrorsum angustato ibique latitudine postica dimidio angustiore, lateribus haud rotundatis ferrugineis, postice aequali, supra scutellum rotundatim producto; elytris basi et lateribus obsolete, epipleuris vero laete ferrugineis, apice oblique truncatis; antennis pedibus abdomineque pallide testaceis.

Longit. $2 \frac{1}{2}$ lin. Latit. 1 lin.

In stagno prope castellum Nicolajevsk peninsulae Kenai, exitu Junii, a D. Holmberg semel lectus.

II. Lapponum Gyllenh. longior et ab illo praeterea variis notis distinctus, superficie tota haud punctata, sed subtilissime et creberrime reticulato-strigulosa, thorace antrorsum magis angustato, lateribus haud rotundatis, postice aequali, minime transversim impresso et punctato, elytris longioribus, postice magis attenuatis, apice oblique truncatis et colore alio.

77. Hydropor planatus : oblongo-ovalis, depressiusculus, dense punctulatus, griseo-pubescens, parum nitidus, niger; thoracis basi latitudine antica sesqui latiore, lateribus obliquis vix rotundatis, elytris nigrobrunneis, posterius pone medium versus apicem rotundatis, stria suturali utrinque leviter impressa; antennarum basi, occipitis margine anguste pedibusque rufo-testaceis.

Longit. 2 lin. Latit. 1 lin. 
Simul cum praecedente a D. Holmberg semel inventus.

II. PLaxo Fabr. admodum similis, ad illum tamen aegre referendus, ob staturam angustiorem, magis parallelam, thoracem antrorsum minus abrupte angustatum, elytra non ante sed longe pone medium posterius angustata et striam suturalem evidenter quamvis leviter impressam.

78. HYd oporus puberús: oblongo-ovalis, subdepressus, crebre reticulato - strigulosus, niger, griseopubescens; thorace lateribus obliquis vix rotundatis; elytris obscure brunneo - castaneis, ante medium apicem versus angustatis, apice ipso rotundato; antennarum basi pedibusque rufo-ferrugineis.

Longit. $1 \frac{1}{3}$ lin. Latit. $\frac{2}{3}$ lin.

Ad ostia fl. Kaktnu peninsulae Kenai, in aquis stagnantibus, exitu Junii, frequens; D. Holmberg.

Ab H. pubescente Gyllenh., a guo aegre distinguitur, differt tamen statura nonnihil angustiore, superficie crebre reticulato-strigulosa elytrisque posterius magis angustatis, striis e punctis seriatis in disco nullis conspicuis.

79. HYd OPORU Nigellus: ovalis, convexus, remotius punctatus, glabriusculus, nitidus, aterrimus, antennis basi rufis; thorace lateribus antrorsum modice angustato, intra basin arcuatim profunde et intra angulos posticos utrinque oblique levius impresso; elytris pone medium apicem versus attenuatis, stria suturali utrinque antice evanescente, posterius sat conspicue impressa.

Longit. $1 \frac{1}{2}$ lin. Latit. $\frac{3}{4}$ lin.

Habitat ad sinum Woskresensk peninsulae lienai, exilu Maji, in stagnis frequens; D. F. Frantienhaeuser. 
Statura omnino II. Americanı Aubé, sed differt colore, punctura profundiore, multo remotiore, thoracis impressionibus et elytrorum stria suturali evidenter conspicua.

\section{G Y R I N I A.}

80. Gyrinus Picipes Eschscholtz: Mannerh. Bullet. de la Soc. de Moscou, 1843. p. 223. 101. ubi diagnosis ita emendanda, ut pro "thoracis et elytrorum margine inflexo nigro-aeneo» substituetur: "thoracis ef elytrorum margine inflexo rufo-aeneo.»

Motschulsky, Hydrocanth. de la Russie, Cat. p. 9.

Var. b. nigra opaca, sutura concolore, versus latera obscure aenea; elytris paullo subtilius striatopunctatis, abdominis segmenti penultimi margine anoque rufescentibus, cetera ut in $a$, quare speciem diversam esse non putarem.

Ilabitat in aquis insulae Kadjak minus frequens; D. Holmberg. Specimen ejusdem varietatis in insula Sitkha caplum, sub nomine G. PICIPEds sibi etiam a D. Eschscholtz mihi olim datum.

\section{H Y,D R O P II I L I A.}

81. Helopirorus consimis: oblongo-ovalis, supra livido-testaceus, glaber, thorace cupreo-nitente, elytris viridi-metallescentibus, subtus piceo-testaceus, dense griseo-pubescens, pedilous ferrugineo-testaceis; thorace antrorsum modice dilatato, angulis posticis obtusatis, anticis haud productis, supra subtilius rugoso-punctato, quinque-sulcato, sulcis latis, secundo et quarto extrorsum valde flexis; elytris maculis paucis fuscis 
signatis, profunde punctato-striatis, interstitiis latioribus, alternis parum elevatioribus.

Var. b. supra obscurius fusco-testacea, vix metallescens.

Longit. $1 \frac{1}{2}$ lin. Latit. $\frac{3}{4}$ lin.

In stagnis ad sinum Woskresensk peninsulae Kenai, exitu Maji vulgaris; D. F. Frankenhacuser. Specimen unicum ad castellum Nicolajevsk ejusdem peninsulae, et alterum prope castellum Constantinovsk ad sinum Nutschek insulae Chtagaluk, mense Juniơ, sub lignis inventa; D. Holmberg.

II. Ixouvito Mannerh. (Bullet. de la Soc. de Moscou. 1852, p. 243. 118.) brevior et praeterea ab illo differt thorace antrorsum latiore, minus rugoso-punctato, angulis anticis (qui in illo acuminati) vix productis, sulcis secundo et quarto extrorsum magis flexis, spatiis interjectis latioribus, elytris magis aequalibus, interstitiisque latioribus, non ut in illo costulatis.

82. II lopuonus angustulus: oblongus, livido-testaceus, supra metallescens, glaber, subtus tenue griseopubescens, pedibus ferrugineo-testaceis; thorace lateribus subrectis, angulis posticis obtusatis, anticis valde productis acuminatis, supra rugoso-punctato, quinque - sulcato, sulcis secundo el quarto extrorsum valde flexis; elytris obsolete fusco-maculatis, profunde et dense punctato-striatis, interstitiis alternis vix elevatis.

Longit. $1 \frac{1}{4}$ lin. Latit. $\frac{1}{3}$ lin.

IIabitat in insula Kadjak et ad sinum Wostresensk peninsulae Kenai rarissime; D. Uolmberg. 


\section{"है.. 74}

Praecedente minor, angustior, thoracis angulis anticis acuminatis productis, elytris densius-punctato-striatis interstitiisque angustioribus distinctus.

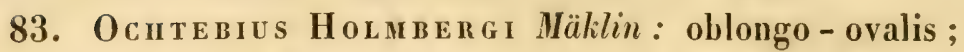
capite cum thorace virescenti-aut interdum rufescentiaeneis, elytris fuscis vel aenescentibus, antennarum basi, palpis pedibusque testaceis; thorace subcordato, dorso antice late transversim impresso punctisque duobus latis postice oblique positis, ad latera arcuatim impresso et in medio canalicula, interdum obsoleta, plerumque punctis duobus latioribus indicata, insculpto, impressionibus omnibus confertim rugulosis; elytris dense minus subtiliter punctato-striatis.

Var. b. elytris fusco-testaceis.

Longit. 1 lin. Latit. parum ultra $\frac{1}{3}$ lin.

In aqua stagnante ad litora maris prope castellum Nicolajevsk peninsulae Kenai a D. Holmberg sat copiose captus

O. manino Payk. Gyllenh. affinis, sed statura corporis magis elongata elytris(jue multo fortius punctato-striatis diversus. ("Mäklin.")

84. Hy nobius fuscipes Linné: ovalis, valde conve. xus, piceo-niger, supra subnitidus, confertissime punctulatus; elytris crenato-striatis, interstitiis secundo, quarto, sex to et oclavo serie irregulari e punctis majusculis notatis, margine dilutiore; palpis pedibusque piceo-ferrugineis.

Longit. 3 lin. Latit. $1 \frac{2}{5}$ lin.

Curtis, Brit. Entom. 2'3. 1.-Stephens Illustr. Mandib. II. p. 128. 2. - Dejean, Cat. 3-ème édit. p 148. - Richard- 
son, Fauna Bor. Amer. IV. p. 119. 169. - Sturm, Fauna X. p. ๖. 2. Tab. CCXVI. - Erichson, Käfer der Mark Brandenb. I. p. 208. 2. - Mulsant, Hist. nat. des Col. de France, Palpicornes. P 122. 3. - Redtenbacher, Fauna Austr. p. 132 - Gaubil, Cat. p. 52. 3. - Bach, Käferfauna. I p. 132. 2. - Cal. Col. Europ 1852. p. 17. Motschulskiy, Ifydrocanth. de la Russie, Cat. p. 10.

Iydrophlus fuscipes De Geer, llém. IV. p. 377. 3. - Olivier, Entom. III. 39. p. 12. 6. Tab. 2. fig. 9. a. b. Gyllenhal, Ins. Suec I. p. 114. 3. - Sahlberg, Ins. Fenn. I. p. 39. 2. - Zetterstedt, Fauna Lappon. I. p. 196. 1. Ins. Lappon. p. 122. 1.

Hydrophues Scarabaeoldes Fabricius, Syst. Ent. p. 288. 4. Syst. El. I. p. 251. 9.-Schönherr, Syn. Ins. II. p. 4. 14.

Drtiscus fuscipes Limn', Fauna Suec. p. 214. 766. Syst. Nat. I. p. 464. 4.

Scarabaeus aquaticus Linné, Fauna Suec. p. 139. 404.

Habitat ad castellum Nicolajevsk peninsulae Kenai, exitu Junii, in aquis stagnantibus minus frequens; D. Holmberg.

85. BEROSUS M A UL OSUS: ovatus, valde convexus; capite profunde punctato, cupreo-aeneo, nitido; thorace brevi, transverse - quadrato, subtilissime punctulato, fulvo - testaceo, in medio macula nigra oblonga lata sublineari, apicem et basin haud attingente; elytris gibbis, inflatis, livido - testaceis, maculis numerosis oblongis nigro - fuscis, hinc inde in fascias undulatas congestis, profunde striatis, striis crenulatis, interstitiis remote punctatis, apice bispinoso, spina suturali breviore; pectore et abdomine piceis, antennis, palpis pedibusque pallide flavo-testaceis.

Longit. 3 lin. Latit. $1 \frac{3}{4}$ lin. 
Enoplurus testellatus Ménétriés: Motschulsky, Hydrocanth. de la Russie, Cat. p. 11.

IIabitat in insula St. Georgii, sec. D. Motschulsky, qui specimen in museo ejus asservatum describere amice mihi concedit. In catalogo citato autem ut insulae Unalaschkae incola designatus.

Ob jam exstantem Berosum testellatum Dej. Cat. 3-ème édit. p. 147. nomen mutavi.

86. CERCY ON LUN I GR UM: ovatum, convexum, nigrum; thorace macula oblonga utrinque intra angulum anticun rufa; elytris fulvo - testaceis, evidentius striatis, striis subtiliter punctatis, interstitiis subtilissime punctulatis, macula pone medium communi, antrorsum lunata suturaque deinde ad apicem nigris.

$V a r . b$. thorace lateribus late rufo-marginato; elytris fulvo - testaceis, immaculatis, sutura apice nigra, pedibus rufescentibus.

Longit. $1 \frac{1}{2}$ lin. Latit. 1 lin.

IIabitat in insulae Kadjak stercoratis, exitu Julii, rarius; Var. $b$. ad castellum Konstantinovsk insulae Chtagaluk, initio Junii, etiam in stercore semel capta; occurrit haec species quoque in insulae Edgecombe; D. Holmberg.

$\Lambda$ sequente thorace elytrisque subtilius punctulatis, his evidentius striatis distinguendum.

87. Cercy on Limatum Mannerheim, Bullet. de la Soc. de Moscou, 1843. p. 260. 189.

Var. d. elytris fulvo-testaceis, macula oblonga, subhumerali, obliqua, infuscata.

IIabitat in insulis Kadjak et St. Pauli; D. Wosnesensley: et 
ad ostia fl. Kaktnu peninsulae Kenai, rarius; 1). Holmberg. Var. c. etiam in insula Kadjak a D. Wosnesensliy et non solum ibidem, sed ad sinum Woskresensk peninsulae Kenai et in insula Chtagaluk a D. Holmberg capta.

\section{S I L P H A L I A.}

88. Negrophorus pollinctor: niger; thorace glabro, lateribus perparum sinuatis, antrorsum vix rotundato - dilatato, disco subtilissime punctulato, intra latera et basin creberrime punctato; elytris subparallelis, latitudine quarta parte longitudinis longioribus, creberrime profunde punctatis, apice subtruncatis, lateribus postice nigro-ciliatis; antennarum clava, membrana nasali minutissima triangulari, elytrorum margine inflexo toto, lobum parum ante medium in paginam superiorem emittente, utroque maculis duabus minutis obsoletis mox pone scutellum transversim positis et fascia postica prope apicem undulata aurantiacis; pectore dense flavo - piloso; tibiis posticis rectis. (Femina.) Mas latet.

Longit. $9 \frac{1}{3}$ lin. Latit. 4 lin.

Habitat in insula Atkha, sec. D. Ménétriés, qui individuum in Museo Acad. Imp. Scient. asservatum mecum benevole communicavit.

N. Mantmino Eschscholtz (Mannerh. Bullet. de la Soc. de Moscou. 1843. p. 251. 168.) valde similis et affinis; femina mihi tantum cognita elytris nonnihil brevioribus, crebrius punctatis, apice subtruncato extus haud rotundato, thorace intra basin et latera minus profunde punctato, haud rugoso membranaque nasali multo minore triangulari fere unice distincta, sed tamen cum specie citato aegre conjungenda. 
89. Necropiorus tardus Motschulsky: niger; thorace glabro, lateribus subrectis, antrorsum haud latiore; capite thoracisque disco subtilissime remote punctulatis, hujus autem partibus lateralibus et postica minus crebre punctatis; elytris parallelis, latitudine quinta parte longitudinis longioribus, profunde remotius punctatis, subbicostatis, apice subsinuatis, lateribus postice castaneo-ciliatis; antennarum clava membranaque nasali minuta obtuse triangulari aurantiacis; elytris maculis parva marginali et duabus ante apicem transversim positis etiam parvis, sanguineis, margine inflexo toto nigro; pectore parcius flavo-piloso; abdomine supra sublaevigato; tibiis posticis rectis. (F e mina.). Mas latet.

\section{Longit. $7 \frac{1}{3}$ lin. Latit. 3 lin.}

Habitat in insula Sitkha, sec. D. Motschulsky, cujus amicitiae individuum in museo ejus asservatum ad describendum debeo.

A N. мавттмо Eschsch. differt magnitudine minore, elytris brevioribus, thoracis disco subtilius punctulato ejusque ambitu laterali et postico remotius punctato, elytrorum punctura etiam remotiore sed profundiore, costisque in utroque binis bene conspicuis, margine inflexo toto nigro et maculis tantum tribus sanguineis.

90. Necropuorus infodiens Eschscholta: niger; thorace glabro, lateribus parum sinuatis, antrorsum modice rotundato - dilatato, disco subtilissime, intra latera et basin crebrius punctulato; elytris latitudine parum longioribus, postice nonnihil dilatatis, subquadratis, profunde punctatis, lateribus postice nigrociliatis; antennarum clava elytrorumque margine inflexo toto et fasciis duabus transversis dentatis sutu- 
ram non attingentibus, aurantiacis; pectore dense flavo-piloso; tibiis posticis rectis.

Mas: capite majore, pone oculos dilatato, canalicula frontali media longiore; membrana nasali aurantiaca spathulata; thorace antrorsum magis dilatato, intra latera et basin minus profunde punctato.

Femina: capite minore, pone oculos haud dilatato, rudimento tantum canaliculae frontalis; thorace antrorsum parum latiore, intra latera et basin profundius rugoso-punctato; membrana nasali aurantiaca minore subsemicirculari.

Var. b. fascia anteriore aurantiaca in maculas tres subdilacerata.

Var. c. loco fasciae anterioris rudimento marginali et maculis duabus disci minutissimis aurantiacis.

Var. d. fascia anteriore omnino deficiente, margine inflexo aurantiaco ante medium in paginam superiorem nonnihil dilata.

Longit. $7-8$ lin. Latit. $3 \frac{1}{4}-3 \frac{2}{3}$ lin.

Habitat in insula Unalaschka, sec. D. Motschulsiy. In ora orientali insulae Kadjak, mense Julio, sub lapidibus et in terra ambulantem (non in cadaveribus) sat frequenter invenit D. IIolmberg. Varietates $b, c$ et $d$. etiam in insula Kadjak captas transmisit D. Ménétriés.

N. maritumo Eschsch, etiam certe propinquus, capitis et thoracis structura omnino eadem, sed maris canalicula frontali distincta, quum in martrmo vix conspicua elytrisque multo brevioribus, minus crebre punctatis ab illo praecipue diversus. 
91. Necropiorus defodiens Mannerheim, Bullet. de la Soc. de Moscou. 1846. Il. p. 63. 10.

N. LUGUBnis Motśchulskì in litteris.

Var. c. fascia anteriore aurantiaca angustiore, dentata, posteriore omnino deficiente.

lisdem locis in insula Kadjak ut praecedens species a D.

Holmberg et una cum Var. $b$ sat frequenter capta.

92. Necrophorus mortúrum Fabricius: niger, supra glaber; thorace vix lateribus sinuato et antrorsum latiore, disco sat crebre, ambitu explanato nonnilil fortius punctato; elytris postice latioribus, subquadratis, apice leviter sinuatis, supra crebre sed minus profunde punctatis, antice fascia transversa latissima in marginem inflexum continuata ibique cum macula subhumerali ejusdem coloris anguste cohaerente maculaque postica magna reniformi, antice dentata, suturam et marginem haud attmgente, aurantiacis; antennarum clava nigra; membrana nasali nulla; pectore dense flavo - piloso, tibiis rectis.

Longit. $7 \frac{1}{3}$ lin. Latit. 3 lin.

Falricius, Ent. Syst. I. p. 248. 5. Syst. El. I. p. 335. 8. Illiger, Käfer Preus. I. p. 354. 4. - Gyllenhal, Ins. Suec. I. p. 260. 4. - Herbst, Col. V. p. 163. 5ै. Tab. L. fig. 6. - Panzer, Fauna Germ. XLI. p. 118. 4. - Schönherr, Syn. Ins. II. p. 121. 8. - Sallberg, Ins. Fenn. I. p. 87. 3. - Erichson, Käfer der Mark Brandenb. I. p. 226. 8. - Zetterstedt, Fauna Lappon. I. p. 168. 3. Ins. Lappon. p. 109. 3. - Sturm, Fauna. XIII. p. 72. 8. Gebler, Bullet. de la Soc. de Moscou. 1847. II. p. 439. 2. - Redtenbacher, Fauna Austr. p. 140. - Gaubil, Cat. p. 55. 15. - Bach, Käferfauna. I. p. 15\%. 8. - Cat. Col. Europ. 1852. p. 17.

Necrophorus Vesplllomes Iterbst, Archiv. p. 32. 3. Scriba, Journ. p. 168. 110. 
Necrophones Vespiclo Var. $\gamma$. Payliull, Fauna Succ. I. p. 325. 2.

Individuum ad fl. Kiwichpakh captum e Museo Acad. Imp. Scient. Petropol. mihi benerole transmisit. D. Ménctriés.

93. Silpia siga $\mathbf{x}$ : ovalis, depressa, nigra, parce fuscopubescens, thorace antrorsum angustato-rotundato, apice emarginato, creberrime punctulato, vario modo sed obsolete inaequali; elytris apicem fere usque parallelis, ad tres partes ambitus reflexo-marginatis, punctis minutis oblongis minus dense seminatis, utroque tuberculo pone medium et lineis tribus elevatis, quarum externa apice incurva ante apicem elytrorum evanescente, intermedia semper supra tuberculum ad apicem producta et interna interdum longe ante apicem deficiente, interdum vero usque in illum producta; antennarum articulo ultimo orbiculato.

Longit. $4 \frac{1}{2}$ lin. Latit. $2 \frac{1}{4}$ lin.

Habitat ad ostia fl. Kaktnu peninsulae Kenai, exitu Junii, in arena litorali rarius; D. Holmberg. Specimen in eadem. peninsula quoque captum amice meum communicavit D. Ménétriès.

S. opaca Linné subsimilis, sed ab illa abunde diversa, statura deplanata, pagina superiore fere glabra parce tantum pubescente, thorace longiore, angustiore, antrorsum angustato, apice emarginato, dense sed non confertim punctato, scutello in medio haud carinato, elytris remote subtilius punctulatis, punctis minutis oblongis signatis, ultra medium parallelis et tantum deinde postice rotundatis, margine reflexo longe ante apicem desinente antennarumque articulo ultimo globoso, qui in S. opaca acuminatus.

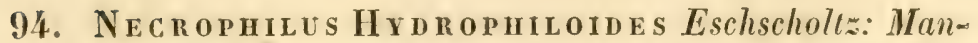
nerh. Bullet. de la Soc. de Moscou. 1843. p. 253.172. 


\section{2}

Var. b. tota nigra, nitida, antennis solum extrorsum tarsisque brunneo-piceis.

N. ATERRimus Motschulsky in litteris.

N. Aten? Motschulsky, Bullet. de la Soc. de Moscou. 18`弓̆. II. p. 363 .

Individuum in insula Sitkha captum benevole transmisit D. Motschulskiy. Species haec etiam in California sat copiose a D. Holmberg inventa.

\section{Y R O S O M A Irénétriẻs.}

Antennae longitudine fere dimidii corporis, extrorsum parum crassiores; articulo primo subcylindrico; secundo triplo breviore subobconico; tertio, quarto et quinto praecedente plus quam duplo longioribus, inter se aequalibus, subcylindricis; sexto, septimo et octavo his paullo brevioribus, etiam inter se aequalibus, subobconicis; nono et decimo praecedentibus longitudine aequalibus, paullo latioribus, sublenticularibus; ultimo his duplo longiore, pyriformi.

Labrum profunde emarginatum, subbilobum.

Mandibulae validiusculae, supra planae, acutae marginatae, in medio transversim impressae, apice acuminatae, incurvae, dente parvo intra apicem vix conspicuo armatae.

Maxillae apice laciniis duabus dentiformibus terminatae.

Palpi maxillares articulo primo brevissimo, secundo nonnihil elongato, extrorsum paullo crassiore, tertio duplo breviore obconico, ultimo iterum paullo longiore subcylindrico, apice rotundato; labiales brevissimae, articulis subcylindricis, fere aequalibus, ultimo apice rotundato. 
Corpus apterum.

Caput thoracis apice parum angustius, oculis haud prominulis.

Thorax cordatus, lateribus anguste marginatis.

\section{Scutellum semicirculare.}

Pedes cursorii, tenues, tarsis omnibus 5-articulatis, quatuor anterioribus articulis validioribus, posticis attenuatis longioribus; maris tarsi antici articulis prima, secunda et tertia valde dilatatis, transversis, compressis, subsemicircularibus, quarto minuto; unguiculis omnibus minutis incurvis.

95. Lyrosoma opaca Ménétriés: elongata, nigro-picea, opaca, ore, antennis pedibusque obscure rufis; capite thoraceque minus crebre punctulatis, hoc latitudine longiore, dorso longitudinaliter tenue excavato, intra latera utrinque longitudinaliter obsolete impresso, angulis posticis subobtusis; elytris oblongo-ovalibus, subconvexis, profunde striatis, striis crenulatis.

$$
\text { Longit. } 2 \frac{2}{3}-3 \text { lin. Latit. } 1-1 \frac{1}{4} \text { lin. }
$$

IIabitat in insulis Atkha et St. Pauli; D. Wosnesensky. In insula Afognak individuum unicum etiam legit D. Holmberg. $\left(^{*}\right)$

(") Zu dieser Gattung gehört ebenfalls Preroloma palbnem Eschscholtz, indem die hier angegebenen generischen Charaktere auf diesen Käfer vollkommen eintreffen. Ich erlaube mir deshalb eine ausführliche Diagnose genannter, bisher wenig bekannter Art hier zu geben:

Lyrosona paluua Eschscholtz: subelongata, rufo-castanea, subnitida; antennis dimidio corporis nonnihil brevioribus; capite thoraceque remote punctulatis, hoc longitudine parum latiore, longitudinaliter in medio et intra latera apicem usque impresso, angulis posticis 
96. Catops bunnipenis: oblongo-ovatus, convexus, crebre subtilissime reticulato - strigulosus, nigro - piceus, griseo - pubescens; antennis thorace vix brevioribus, ferrugineo - testaceis, clava parum incrassata fusca, articulo octavo minutissimo; thorace longitudine sesqui latiore, lateribus modice rotundatis, antice latitudine basis haud angustiore, angulis omnibus subrotundatis; elytris obscure castaneis, apice subacuminatis, stria suturali leviter exarata; pedibus piceotestaceis.

Longit. $1 \frac{3}{4}$ lin. Latit. $\frac{3}{4}$ lin.

IIabitat ad fl. Tschunuktnu peninsulae Kenai, exitu Junii, in cadaveribus sat frequens; D. F. Frankenhaeuser.

C. Cadaverivo Eschscholtz longior, posterius magis attenuatus, praeterea antennis tenuioribus thoraceque multo latiore, breviore, antrorsum haud angustato, ab illo dignoscendus.

97. Catops luridipenis : ovatus, convexus, crebre subtilissime reticulato-strigulosus, nigro-piceus, griseo-pubescens; antennis thorace nonnihil longioribus, crassiusculis nigris, articulo octavo minuto; thorace longitudine fere duplo latiore, lateribus rotundato, antice latitudine basis haud angustiore, angulis

obtusis; elytris ovalibus, subconvexis, profunde striatis, striis crenatis.

Longit. 2 lin. Latit. $\frac{5}{6}$ lin:

Pteroloma paluduar Eschscholtz, Zoologischer Atlas. I. p. 7. Tab. VIII. fig. 8.

Habitat in peninsula Kamschatka sub lapidibus et lignis deciduis in terra sicca; D. Eschscholtz. Specimen ibidem a D. A. Collan captum e musco suo ad describendum amice transmisit D. Mäklin. 


\section{5}

omnibus rotundatis; elytris obscure castaneis, apice obtusis rotundatis, stria suturali leviter exarata; tarsis rufis.

Longit. $1 \frac{1}{2}$ lin. Latit. $\frac{5}{4}$ lin.

Ad rivulos ff. Tschunuktnu peninsulae Kenai, mensibus Julio et Augusto, in cadaveribus a D. F. Frankenhacuser haud frequenter lectus.

Praecedenti aflinis, sed brevior, practerea antennis crassioribus, thorace breviore, lateribus magis rotundato elytrisque apice rotundatis distinctus.

98. Catops cadaverinus Eschscholt:: Mannerh. Bull. de la Soc. de Moscou. 1843. p. 254. 173.

$V a r . b$. ferrugineo-testacea, capite fusco, thoracis disco antennisque infuscatis, elytris pallide lividotestaceis, postice nonnihil obscurioribus.

In insulae Afognak cadaveribus, mense Augusto, rarius invenit D. Holmberg, qui hanc speciem etiam in California cepit. A D. F. Frankenhaeuser e partibus interioribus peninsulae Kenai quoque allatus.

99. Colon maxicolus: oblongo-ovatus, convexus, rufo-castaneus, brevissime fulvo - pubescens, subtilissime confertim punctulatus; antennis brevibus, extrorsum sensim modice incrassatis; thorace maximo, subquadrato, angulis posticis obtusis; elytris a basi ad apicem sensim angustatis, stria suturali impressa; pedibus validis, muticis.

Longit. $1 \frac{1}{2}$ lin. Latit. $\frac{2}{3}$ lin.

Habitat ad sinum Woskresensk peninsulae Kenai rarissime;

D. Holmberg. 
100. Colon Clavatus: oblongus, convexus, brunneus, fulvo-pubescens, subtiliter punctatus; antennis longitudine thoracis, basi ferrugineo-testaceis, clava magna, oblonga, fusca; thorace antrorsum valde rotundato - angustato, basi truncato, angulis posticis nonnihil supra elytra productis, subrectis; elytris elongatis, a basi ad apicem sensim angustatis, stria suturali minus arcuatim impressa; pedibus mediocribus, piceo-testaceis, muticis.

Longit. 1 lin. Latit. $\frac{x}{3}$ lin.

Ad rivulum fl. Tschunuktnu peninsulae Kenai, mense Augusto, excipulo e gramine a D.F. Frankenhacuser semel captus.

C. rusculo Erichs. statura et punctura affinis, sed differt antennarum clava multo majore, magis incrassata et pedibus obscurioribus.

\section{A E G I A L I T E S Eschscholtz.}

Antennae capite cum thorace breviores, sub angulis anticis clypei insertae, moniliformes; articulo primo ceteris majore pyriformi, valde intumido; secundo triplo minore, subgloboso; tertio sesqui longiore subobconico; \-8 magnitudine secundi breviter subobconicis; 9 et 10 paullo latioribus, sublenticularibus; ultimo praecedente haud latiore sed paullo longiore, apice conico-acuminato.

Labrum brevissimum, apice levissime emarginatum, linea transversa exarata insculptum.

Mentum transverse quadratum, apice emarginatum.

(Mandibulas el maxillas occultas in speciminibus cuique museo unicis examinare mihi non licuit.) 


\section{7}

Palpi maxillares articulo primo brevissimo, secundo latitudine sesqui longiore obconico, tertio iterum paullo breviore etiam obconico, ultimo subovato, inflato; labiales tenues minuti, articulis primo et secundo aequalibus subcylindricis, ultimo paullo crassiore ovato.

Caput cum oculis thorace vix angustius; clypeo brevissimo, apice truncato angulis rotundatis, intra apicem transversim impresso, margine parum elevato; fronte longitudinaliter profunde biimpressa; oculis parvis rotundatis, nonnihil prominulis.

Thorax latitudine vix brevior, modice convexus, apice truncatus, medio baseos emarginatus, lateribus sulırectis, angulis omnibus rotundatis.

Scutellum haud conspicuum.

Elytra ovata, modice convexa, basi thorace latiora, humeris rotundatis, deinde usque pone medium sensim dilatata, angustissime marginata, apice late rolundata.

Alae nullae.

Pedes subelongati; femoribus nonnihil intumidis, tibiis omnibus arcuatis; tarsi quatuor anteriores quinque postici quadri-articulati, articulo ultimo praecedentibus simul sumtis multo longiore, valido, apice intumido . unguiculis acutis, incurvis, ceteris articulis inter se contractis, compressis, subtus hirsutia fulva spongiosis.

Ubi hoc genus in systemate coleopterorum ponendum sit, difficile dictu. Antennarum et palporum structura ScviMAENIS affinitatem exhibet; capitis et pedum vero forma ut et tarsorum articulis heteromeris HeLopis potius adnumerandum erit; ob tarsorum articulum ultimum elongatum D. Motschulsky insectum nostrum cum MACro- 
sxcus jam collocavit. Sed, si etiam pedes accurate examinaveris, certe concedis, nos aliquam habuisse rationem Aeginditem in vicinitate Scydmanorum retinere.

101. Aegialites Debilis Eschscholtz: nigro-piceus, nitidus; capite thoraceque punctis paucis sparsim irregulariter impressis, hoc longitudinaliter profunde canaliculato; ely tris striato-subsulcatis, pilis rigidis atris sparsim obsitis, striis in fundo irregulariter rugosopunctatis, ante apicem vario modo coeuntibus; pedibus rufescenti-piceis, femoribus obscurioribus. (")

$$
\text { Longit. } 1 \frac{2}{5}-2 \text { lin. Latit. } \frac{2}{3}-1 \text { lin. }
$$

(*) Das einzige Stiick des räthselhaften Aegramtes denulus Eschscholt der friheren Dejean schen Sammlung gehört gegenwärtig dem Herrn Marchese Spinola in Genua. Um die Identität dieses Käfers einigermassen auszumitteln, schrieb ich an diesen ehrwürdigen Veteranen unserer entomologischen Wissenschaft, der auch meinem Briefe Folge leistend die Gewogenheit mir erwies, eine Zeichnung des AegramTES DEBus anfertigen zu lassen, die er mir später schickte, mit folgenden schriftlichen Erörterungen begleitet: al'exemplaire unique "de la collection Dejean est dans un état déplorable. Il a perdu quel"ques pattes et les antennes hors les deux premiers articles. La bou"che est masquée par je ne sais quelle pate ou masse glutineuse qui «en dérobe toutes les parties à la vue hors les extrémités des palpes "maxillaires, qui ont trois articles en evidence, mais qui en ont "peut-ètre davantage." Nach dieser Zeichnung wären wohl der Kopf, die Augen, die Oberlippe, die Maxillar-Taster und die gekrümmten Beine denselben Theilen der von mir jetzt beschriebenen Art vollkommen entsprechend; das zweite Glied der Fühlhörner ist in der Zeichnung etwas kürzer, das Halsschild und die Flügeldecken sind auch mehr langgestreckt als bei den von mir untersuchten Exemplaren, was aber besonders auffält sind die in der Zeichnung zugespitzten Deckschilde. Allein da die Zeichnung nach einem in manchen Theilen zerstiummelten stiicke gemacht wurde, hätte wohl auch die nöthige Genauigkeit hier und da den Zeichner entgehen können, und indem nebst der Grösse doch so viele Theile, wie Kopf, Auren, Taster, das erste und beinahe das zweite Glied der Fühlhörner, 
Dejean, Cat. 3-ême édit. p. 131.

Elosoma? Camornica Motschulsky, Bullet. de la Soc. de Moscou. 18น5. I. p. 33. 83.

Specimina tria examinavi; unum ut Californiae incola designatum, in museo I). Motschulstiy exstans, alterum in insula Sitkha a D. Ferd. Sahlbery captum et tertium a 1). Holmberg ad sinum Woskresensk peninsulae Kenai lectum.

\section{S T A P H Y L I N A.}

102. MYrmedonia angularis Mäklin: confertim subtilissime punctata, fusca, antennarum basi, thoracis lateribus, pedibus elytrisque testaceis, his sutura, bitsin versus latius angulisque, apicalibus oblique late infuscatis; thorace transverso, obsoletius canaliculato, basi leviter transversim foveolato.

Longit. $1 \frac{1}{2}$ lin. Latit. $\frac{2}{5}$ lin.

IIabitat in peninsula Kenai; in interiore parte et ad sinum Woskresensk a D. F. Frankenhacuser rarissime inventa. ("Mäklin ")

103. I о ма L depressa, nigra, geniculis tarsisque testaceis; antennis filiformibus, capite cum thorace longioribus; thorace basin versus angustato elytrisque subtiliter coriaceis, vix visibiliter confertissime punctulatis; illo longitudi-

Beine, die Furche in der Mitte des Halsschildes und die Streifen der Flügeldecken als übereinstimmend hervorstehen, habe ich mich endlich entschlossen, die Meinung meines verehrten Freundes Motschulsky zu theilen und folglich seine Elosoma? Califorvica als identisch mit dem Aegraltes debils Eschsch. hiemit zu rerkündigen. 


\section{0}

naliter obsolete canaliculato; his thorace tertia parte longioribus; abdomine dense evidentius punctato.

Longit. fere 2 lin. Latit. parum ultra $\frac{1}{3}$ lin.

In insula Kadjak a D. Irolmberg rarissime capta.

Statura corporis H mantmas Eschsch. (Mannerh. Bullet. de la Soc. de Moscou. 1843. p. 224. 102.) affinis et similis, sed multo major, minus depressa, thorace longiore et in aliis diversa. Ad div. I. operis Cel. Erichson referenda. Variat pedibus totis fusco-testaceis. In individuo unico thorax punctis duobus impressis signatus est. ("Mäklin n.)

104. Hомадота litoralis Mäklin: depressa, nigra, opaca, pube grisea subrigida dense vestita, elytris flavis, lateribus latius, basi angustius nigro - fuscis, pedibus piceis, tarsis testaceis; antennis crassiusculis, capite cum thorace brevioribus, thorace leviter transverso, posterius ante basin latissime transversim impresso, confertim subtilissime punctulato; elytris thorace vix longioribus, dense at minus confertim et paulo evidentius quam thorax punctatis; abdomine vix nitido, parcius et parum profunde punctato, segmento ultimo subtiliter densius granulato.

Longit. fere 2 lin. Latit. ultra $\frac{1}{3}$ lin.

Habitat in insula Kadjak; a D. Holmberg rarissime inventa. ("Mäklin.")

105. Homalota fucicola Mällin.

Taciyusa fucicona Mäklin: Mannerh. Bullet. de la Soc. de Moscou. 18ว̈2. I. p. 306. 32.

In insula Kadjak sub lapidibus in vicinitate maris a $\mathbf{D}$. Holmberg copiose lecta. 


\section{1}

Haec species cum gen. Tacurusı corporis statura quodammodo conveniens, ob structuram tarsorum gen. НомLoraE potius erit adscribenda. ("Mäklinn.)

106. Hонадота уазта Mälilin: latiuscula, nigra, nitida, antennarum basi pedibusque testaceis, elytris piceo - testaceis, angulis apicalibus nomnihil nigricantibus; antennis capite cum thorace vix longioribus, apicem versus sensim incrassatis ; thorace transverso, subtiliter obsoletius punctato, ante scutellum obsolete longitudinaliter impresso; elytris thorace paullo longioribus, dense ef multo evidentius quam thorax punctatis; abdomine segmentis primis parcissime subtiliter punctatis, ultimis fere omnino laevigatis.

Longit. $1 \frac{1}{2}$ lin. Latit. fere $\frac{1}{2}$ lin.

A I). Holmberg in insula Sitkba semel capta.

Ad div. 2. operis Cel. Erichson referenda. ("Miililin.")

107. Ox Y P D i r A A Mälklin: nigra, opaca, subtilissime et confertissime punctulata, fusco-pubescens, elytris obscure fuscis, pedibus testaceis; thorace convexo, aequali.

Var. b. elytris rufo-piceis.

Longit. $1 \frac{1}{3}$ lin. Latit. $\frac{x}{3}$ lin.

In insula Sitkha a D. Holmbery semel, et in parte interiore peninsulae Kenai a D. F. Frankenhueuser rarissime inventa.

O. opacae Grac. statura corporis admodum similis, sed paullo angustior et multo minor. ("Mülilin.")

108. Aleochara bi a Culata Gravenhorsl: nigra, nitida, antennarum basi pedibusque piceis; elytris thorace 
subbrevioribus, macula apicali testacea; thorace lateribus minus dense, dorso irregulariter biseriatim, abdomine supra confertim punctato.

Longit. $1 \frac{1}{2}-1 \frac{2}{5}$ lin. Latit. ultra $\frac{1}{3}$ fere $\frac{1}{2}$ lin.

Gravenhorst, Micropt. Brunsv. p. 187. 3. Monogr. Mier. p. 170. 58. - Say, Acta Acad. nat. Scienc. Philadelph. III. p. 157. - Erichson, Gen. et Spec. Staph. p. 167.

In insula Afognak a D. Holmberg rarissime capta.

Quamquam punctura thoracis non omnino cum descriptione a Cel. Erichson, data convenit, tamen eandem ad hanc speciem referre coacti fuimus, quum in musaeo Universitatis Alexandrinae individuum, ad Novum Eboracum a D. Tengstroem captum, hisce borealibus etiam magnitudine et ceterum simillum sit. Ab A. NITIDA Grav. Erichs. thorace lateribus paullo densius punctato diversam esse videtur. ("Mäklin.w)

109. Gropopaena geniculata Mäklin: nigra, nitida, subtiliter at creberrime punctulata, antennarum basi, geniculis tarsisque testaceis, ano piceo; thorace basi anguste marginato, ante scutellum interdum utrinque impresso.

Longit. $\frac{5}{6}-1$ lin. Latit. parum ultra $\frac{1}{4}$ lin.

Ad sinum Nutschek in insula Chtagaluk a D. Holmberg rarissime inventa. ("Mälilin. ")

110. Tacuinus nigriconnis Mannerheim: Bullet. de la Soc. de Moscou. 1843. p. 225. 106.

II as: abdominis segmento superiore sexto quadridentato, dentibus intermediis parum longioribus, spiniformilbus; segmento inferiore quinto apice leviter emarginato at profunde impresso, impressione late spongioso, sexto bifido, laciniis elongatis. 


\section{3

In insula Sitkha a D. Holmberg captus. Mas antea a me e coll. Ill. Com. Mannerheim descriptus (Bullet. de la Soc. de Moscou. 1852. I p.310. 43.) ad speciem proxime subsequentem potius referendus. ("Mäklin. ")

111. Tacinus insta B I Is Mäklin: niger, nitidus, thoracis margine laterali et basali angustissime, elytris puncto humerali et basin versus obsoletius pedibusque rufo-castaneis; elytris thorace plus quam dimidio longioribus, dense evidentiusque punctatis.

Mas: abdominis segmento superiore sexto quadridentato, dentibus intermediis parum prominulis, minus acutis; segmento inferiore quinto emarginato, margine infra sinum anguste spongioso.

Femina: abdominis segmento superiore sexto tridentato, dentibus fortiter elongatis, fere spiniformibus; segmento inferiore quinto apice integro.

Var. b. elytris pedibusque dilutius rufo-castaneis, illis ante apicem nigricantibus.

Var. c. elytris pedibusque castaneo-rufis.

Longit. $1 \frac{3}{4}-2 \frac{1}{4}$ lin. Latit. $\frac{3}{4}-1$ lin.

IIabitat in insula Kadjak; D. Holmberg. Individua ibidem collecta D. D. Ménétriés et Motschulsky ad describendum quoque transmiserunt.

A praecedente, cui antennarum colore et in aliis quodammodo símilis est, magnitudine plerumque multo minore, elytris fortius punctatis, abdominis structura ut et colore evidenter diversus. ("Maklin. n) 
112. Tachinus frigidus Erichson: Mannerh. Bullet. de la Soc. de Moscou. 1843. p. 226. 107. 1852. I. p. 310.44 .

Habitat etiam in insula Afognak et in peninsula Kenai; D. Holmberg.

Var. b. thoracis limbo omni, laterali latius elytrisque testaceis, his apicem versus infuscatis.

In peninsula Kenai ad sinum Woskresensk a D. F. Frankenhaeuser inventa:

A T. Propinquo Mannerh. (Bullet. de la Soc. de Moscou. 1843. p. 226. 108.) haec varietas magnitudine majore et thorace angustiore, lateribus minus rotundató-amplicatis distinguitur.

Obs. Dentes intermedii abdominis segmenti superioris sexti maris saepe minus prominentes, apice obtusiores, in ceteris vero omni modo inter se conveniunt. ("Mäklin n.)

113. Tachun us a terus Ménétriés: subelongatus, niger, nitidus, ore (antennis?) pedibusque rufis; elytris planiusculis, thorace parum longioribus, omnino fere laevigatis.

Mas latet.

Femina: abdominis segmento superiore sexto trifido, laciniis acuminatis, intermedia breviore, basin versus paullo latiore.

Longit. $2 \frac{1}{5}$ lin. Latit. $\frac{4}{5}$ lin.

Individuum unicum mutilatum, in insula St. Georgii captum, ad describendum transmisit D. Ménétriés.

Statura corporis subelongata et abdomine fere parallelo

'T. elongato Gyllenh. subsimilis, sed elytrorum brevitate, punctura eorum omnino fere deficiente et abdominis structura satis diversus. ("Mäklin.")

114. MyсетоPORUS Insignis Mäklin: oblongus, niger, nitidus; thorace disco laevissimo, paullo ante margi- 


\section{5}

nem basalem punctis quatuor transversim positis impresso, segmentorum marginibus abdominis elytrisque rufis; his thorace sesqui longioribus, in medio macula magna discum totum fere occupante margineque laterali nigris, prope seriem dorsualem punctis duobus impressis; antennis capite cum thorace paullo brevioribus, apicem versus leviter incrassatis pedibusque rufo-testaceis; femoribus posticis infuscatis.

Longit. $2 \frac{\pi}{4}$ lin. Latit. 1 lin.

In insula Afognak a D. IIolmberg semel tantum inventus. ("Mäklin.")

115. Mycet oporus rigrans Mällin: subelongatus, niger, nitidus, antennarum basi, thoracis margine basali, elytrorum limbo apicali pedibusque testaceis; antennis apicem versus modice incrassatis; articulis penultimis transversis; thorace punctis quatuor multo intra apicem transversim positis et codem numero paullo ante marginem basalem impresso; elytris serie punctorum dorsuali simplici.

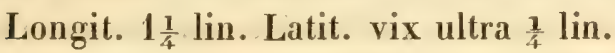

Specimen singulum in interiore parte peninsulae Kenai legit D. F. Frankenhaeuser. ("Mälilin.")

\section{TRICHOCANTHUS Motschulsky.}

Antennae in individuo descripto desunt.

Labrum transversum, bilobum, corneum.

Mandibulae validae, falcatae, in medio fortiter bidentatae, basi lacinia elongata, ciliata instructae. 
Palpi maxillares articulo secundo elongato tertio duplo fere longiore, tertio quartoque longitudine inter se aequalihus; labiales articulis omnibus longitudine aequalibus.

Ligula apice levissime emarginata, paraglossis eam longe superantibus.

Pedes intermedii basi approximati.

Insectum apterum generi Ocypo magis quam Stapiyuivo affine esse videtur.

116. Trichocantues variegatus Motschulsky: pallide testaceus, macula transversali capitis, punctis duobus confluentibus in collo, linea transversali antica arcubusque quatuor in thorace, cingulo latiore in elytris, punctis biseriatim dispositis in abdomine, tibiis basi et mandibularum apice nigris; elytris thorace duplo fere brevioribus,

Longit. $5 \frac{1}{2}$ lin. Latit. $2 \frac{1}{2}$ lin.

Motschulsky, Etudes entom. 1852. p. 78.

Individuum unicum, ut in continenti Americes borealis occidentalis captum, ad describendum benevole transmisit D. Motschulsky. ("Mäklin.")

117. Philontius aterrimus Gravenhorst: elongatus, niger, antennis basi piceis, pedibus testaceis; capite ovato; thorace seriebus dorsualibus sexpunctatis; elytris thorace paullo longioribus, minus dense sat fortiter punctatis.

Longit. 2 lin. Latit. parum ultra $\frac{1}{3}$ lin.

Erichson, Käfer der Nark Brandenb. I. p. 476. 45. Gen. et Spec. Staphyl. p. 492. 109. - Heer, Fauna Col. Helv. 
p. 271. 50. - Redtenbacher, Fauna Anstr. p. 706. Gaubil, Cat. p. 252. 75. - Catal. Col. Europ. 1852. p. 26.

Stapiy unes ateramus Gravenhorst, Micropt. p. 41.62. Gyllenhal, Ins. Suec. II. p. 349. 65. - Salliberg, Ins. Femr. I. p. 331. 62. - Zetterstedt, Fauna Lappon. I. p. 79. 31. Ins. Lappon. p. 65. 41. - Dejean, Cat. 3-ìme édit. p. 70. - Boisduval et Lacordaire, Fauna Ent. Paris. I. p. 408. 43.

Carius aternimus Mannerheim, Brachelytr. p. 32. 5.

Gynonypus atennimus Nordmann, Symbol. p 126. 31.

Gannius atennisos Stephens, Manual of Brit. Col.

In insula Kadjak a D. Holmberg semel tantum inventus. ("Mäklin.")

118. Phicontud femoralis Mäklin: niger, opacus, elytris fuscis, mandibulis, femoribus omnibus tarsisque anticis flavo - testaceis; capite thoraceque creberrime at obsoletius punctato, hoc carinula in medio interrupta; etytris thorace dimidio longioribus abdomineque confertissime subtilissimeque punctulatis.

Longit. $2 \frac{1}{4}$ lin. Latit. parum ultra $\frac{1}{3}$ lin.

In insula Kadjak specimen unicum cepit D. Holmberg.

Ad div. 8-vam hujus generis in opere "Genera et Species Staphylinorum " auct. Erichson referendus. ("Mäklin.")

119. Quedius rufipennis Mäklin: niger, nitidus, antennarum basi, elytris, segmentorum abdominalium marginibus pedibusque rufis; thorace lateribus parum rotundato; elytris thorace tertia parte longioribus, glabris, sublaevibus.

Longit. 4 lin. Latit. $\frac{5}{6}$ lin. 
Ad sinum Woskresensk peninsulae Kenai a 1). F. Frankenhaeuser medio mensis Maji semel captus.

Qu. Laevigato Gyllenh. Erichs similis et aflinis, sed thorace elytrisque paullo longioribus, illo angustiore, lateribus vix rotundato et abdomine adhuc subtilius punctato; a Qu. plagiato $\left({ }^{*}\right)$ et congipenni Mannerh. discedit magnitudine majore et statura corporis latiore. ("Mälilin.")

120. Quedus a enescens Málin: Mannerh. Bullet. de la Soc. de Moscou. 1852. I. p. 315. 55.

Habitat etiam in insulit Edgecombe; D. IIolmberg.

Var. c. nigra, nitida, elytris nigro-aeneis, antennis pedibusque nigro - piceis, illis basi, his geniculis, abdominisque segmentorum marginibus anguste rufis.

In insula Kadjak a D. Holmberg inventa. - Obs. D. Ménétriés individuum hujus speciei in insula Sitkha captum nomine varietatis Qu. Transparentis Molsch. transmisit, sed descriptio a v. nob Motschulsky (Bullet. de la Soc. de Moscou. 184.5 p. 358. 34) data: "fronte inter oculos punctis sex vel octo impressa" cum specie nostra non convenit. Magnitudine et habitationis loco etiam inter se differunt. ("Mäklin.")

121. Quedius subin BAtus Motschulsky: fusco-brunneus, capite abdomineque nigris, antennarum basi, macula humerali ut et limbo laterali et apicali elytrorum, segmentorum abdominalium marginibus pedibusque testaceis; elytris minus nitidis, thoraci longitudine aequalibus, dense subtilius punctatis.

(") Cl. Hochhuth novissimis temporibus aliam hoc nomine descripsit speciem, Qu. phagatum Chaudoir, quae denominatio igitur commutanda est. ("Mäklin.») 


\section{9}

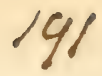

Var b. thorace dilutius brunneo.

Longit. $2 \frac{1}{2}-2 \frac{3}{4}$ lin. Latit. $\frac{2}{3}$ lin.

Individua quaedam in insula Kadjak invenit D. IIolmberg: varietatis $b$ unicum ibidem captum conservato nomine ad describendum transmisit D. Motschulsky.

Ilabitat quoque in Sibiria orientali; D. Pippingsliöld.

Qv. maunonuro Grar. Erichs. simillimus, sed colore saturatiore, antennarum articulis brevioribus et abdomine minus crebre at paululum fortius punctato diversus ("Mälilin.")

\section{LIPAROCEPHALUS Mällin.}

Antennae apicem versus nonnilil incrassatae, articulis penultimis leviter transversis.

Labrum transversum, integrum, margine setosum.

Mandibulae longae, falcatae, acutae, medio fortiter unidentatae, inter dentem et apicem denticulis nonnullis parvis armatae.

Naxillae malis interne barbatis, exteriore falcata.

Palpi maxillares articulo ultimo minimo, subulato; labiales filiformes, articulo secundo tertio paulo longiore.

Caput thorace majus, sed tamen non latius.

Thorax subcordatus, basin versus angustatus.

Elytra brevissima.

Alae nullae.

Tarsi articulo quarto minimo, simplici.

Genus hoc insigne, Paedernas adscribendum, generi Stulico statura corporis fortasse proximum est. 
122. L I P A R C E P II A U S R EV IPEN I Mäklin: niger, opacus, creberrime at vix evidenter punctulatus, pube fusca subtili dense vestitus, maxillis, antennarum basi tarsisque rufis; antennis capite cum thorace paulo brevioribus.

Longit. 2 lin. Latit. $\frac{2}{3}$ lin.

In insula Chtagaluk ad castellum Constantinovsk, sub fucis e mare rejectis, a D. Holmberg bis tantum captus. ("Mäklin.")

123. St te u S im м R in a tus Mällin: cylindricus, niger, nitidus, palpis fuscis, basi testaceis, parce profunde punctatus; capite in medio carinato, utrinque latius sed minus profunde impresso; thorace basin versus subtiliter obsoletiusque canaliculato; elytris thoracis longitudine; abdomine immarginato, parcius subtiliusque punctato; tarsis articulo quarto simplici.

Longit. 1 - $1 \frac{1}{4}$ lin. Latit. $\frac{1}{3}$ lin.

Habitat in insula Kadjak rarissime; D. Holmberg.

Individuis minoribus Sr. Nignitulr Gyllenh. magnitudine aequalis, sed punctura parciore et fortiore omnino diversus. ("Mäklin.")

124. Stenus congener Mälilin: plumbeo-niger, densius profunde punctatus, subtiliter albido-pubescens, palpis articulo primo testaceo; fronte planiuscula; thorace subtiliter at distincte canaliculato; elytris thorace paulo longioribus; abdomine marginato, crebrius subtiliusque punctato; tarsis articulo quarto simplici.

Longit. $1 \frac{1}{5}$ lin. Latit. $\frac{1}{5}$ lin.

In insula Kadjak a D. Holmberg semel captus. 
Intermedius quasi inter St. aemulea Erichs. et St. CaylicuL.iтем Gyllenh., illo densius at subtilius, hoe e contrario nonnihil parcius ef profundius punctatus. ("Mäklin.")

125. Brenes a boxotatus ä̈lilin: niger, fusco-pubescens, geniculis, tibiis posticis tarsisque omnibus testaceis, elytris nonnihil aenescentibus, angulis apicalibus late inaequaliter albis; thorace obsoletius parciusque punctato, subtiliter canaliculato; elytris thorace plus quam dimidio longioribus, creberrime subtiliter punctatis.

Longit. 2 lin. Latit. $\frac{1}{3}$ lin.

Ad castellum Nicolajersk peninsulae Kenai a 1). Holmberg semel inventus. ("Mällin.")

126. OLISTHARUS MEGACEPHALs Zetterstedt: elongatus, rulo-castaneus, nitidus, capite thoracis latitudine segmentisque singulis abdominis basin versus nigris; abdomine parcius obsoletiusque puictato.

Longit. $2 \frac{1}{3}-3$ lin. Latit. $\frac{3}{4}-\frac{1}{3} \ln$.

Erichson, Gen. et Spec. Staphyl. 1. 8'3. 1. - Iteer, Fauna Col. Helv. p. 173. 2. - Cat. Col. Europ. 18ว2. p. 29.

Onamum megacemalum Zotterstedt, Fauna Lappon. I. p. 56. 17. Ins. Lappon. p. 54. 25.

In interiore parte peninsulae Kenai a 1). $F$. Frantienhueuser rarissime eaptus; benignitati D. Pippingsküld specimen ad Ajan Sibiriae orientalis inventmm debeo. Onni modo rum individuis a memet in Lapponia huusamoensi lectis conveniunt. ("Mäklin.")

127. Lesteva Fescoyiga Motschulsty: nigra, pube grisea brevi dense restita, confertim subtiliter punetata, palpis, antemnis pedibusque fuscis, palporum arti- 
culo ultimo, femoribus basin versus, tibiis apice tarsisque rufis; thorace subcordato, lateribus explanato, paulo ante medium fortiter dilatato, ibique puncto majusculo utrinque impresso.

Longit. 3 lin. Latit. $1 \frac{1}{5}$ lin.

Pulaeoptenus fusconiger Motschulsky, Etudes entom. 1852. p. 78 .

Individuum unicum vario modo mutilatum, ut in insula Unalaschka captum, ad describendum benevole transmisit D. Motschulskiy.

Statura corporis admodum quidem singularis, sed vix proprii generis; convenit enim sat bene cum Lesteva palporum maxillarium forma et capite inter oculos punctis duobus impresso. Ad Onasinos certe referenda est, quum caput ocellis duobus instructum sit. ("Mäklin.n)

128. Acidota Frankenineuseri Mäklin: elongata, rufa, antennis pedibusque rufo-testaceis; thorace subquadrato, latiludine parum breviore, parcius punctato; elytris thorace vix sesqui longioribus, parce, fortiter profundeque punctatis; abdomine parcius punctato.

Longit. $1 \frac{5}{4}-2$ lin. Latit. $\frac{2}{5}$ lin.

In interiore parte peninsulae Kenai a D. F. Frankenhaeuser rarissime inventa.

Thorace longiore $\Lambda$. chenatae Fabr. Erichs. el A. quadatae Zetterst. (Ins. Lappon. p. 53.) affinis, huic simillima et non nisi punctura, praesertim abdominis, multo parciore diversa. ("Mäklin.")

129. O соривим датем Ménétriés: latiusculum, nigrum, antennarum basi, thoracis marginibus, elytrorum limbo apicali pedibusque rufo-piceis; thorace transverso, parcius punctato; elytris parcius fortiter punctatis. 
$V a r . b$. antennis, thoracis marginibus, elytris pedibusque dilutius rufo-castaneis; elytris parcius quam in $a$ punctatis.

Var. c. thorace elytrisque rufo-ferrugineis, his adhuc parcius punctatis.

Longit. 2 lin. Latit. 1 lin.

Individuum unicum in insula St. Georgii captum ad deseribendum benigne transmisit D. Ménétriés; varietatis $b$. duo exemplaria ad caslellum Nicolajevsk peninsulae Kienai cepit D. Holmberg et singulum Var. $c$ prope fl. Tschuniten in eadem peninsula D. F. Frankenhaeuser ("Mälilin.n)

130. Ozopund panvelua Mälin : ferrugineo - testaceum; thorace subquadrato, subtilius punctato, basin versus nonnihil angustato, lateribus ante angulos posticos leviter sinuatis; clytris fortiter punctatis.

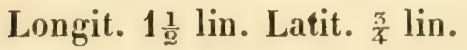

Ad lacum Thkujabna in peninsula Kenai, initio mensis Junii, sub lapide a D. F. Franlienhaeuser semel lectum.

O assimur Payk. proximum, sed paulo minus; distinguitur facillime thorace paulo angustiore, subtilius punctato, lateribus ante basin subsinuatis et angulis posticis, etsi obtusis, tamen nonnibil prominulis. ("Mäklin.")

131. Olophum convexum Mäklin: oblongum, convexum, nigro-piceum; thorace latitudine parum breviore, dense profundius punctato, angulis posterioribus late rotundatis; elytris apice exacte truncatis, angulo exteriore minus late rotundato.

Longit. $2 \frac{2}{3}$ lin. Latit. $\frac{\frac{\pi}{6}}{6}$ lin. 


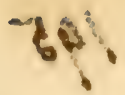

\section{4}

4). F. Funknlucuser in interiore parte peninsulac Kena: rarius captum.

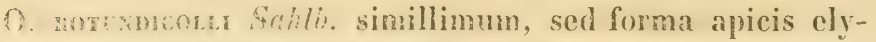

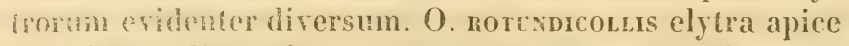
ahiogiatim, licel bbtuse, sunt rotundata, angulo exterione mulfo latione. Discedit nostra specios quoque punclura paulo profundiore el parciore. ("Mälitin.,)

132. 0) בon

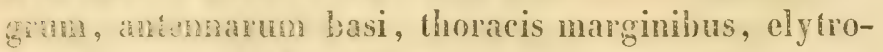

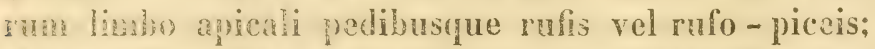
thorare masin versus leviler angustato, hateribus postice subsinualo, densius, punctato.

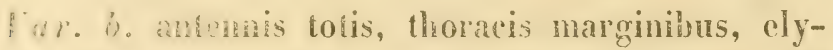
Anis, pudisss anome late piceo-rufis; elytris margine apicali rufo-testaceo.

Fr. c. antinuis, dsorace, elytris, ano pecibusque rufo - brunneis.

Longुit. $1 \frac{3}{4}-2$ lin. Latit. $\frac{1}{5}$ lin.

[1. Bifat in yorbbus interoribus peninsulac Kenai rarius:

1). F. Frantienhaeuser. Var $b$. et $c$. ad ostia fl. Kaktnu cjusdem peninsulate cepit D. Holmberg.

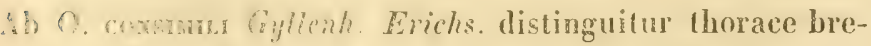
, inere. Hisin versus magis angustato, et ninus conferlim pranctato. ("Mälilin.")

133. DELIPHIUH REVICOLLE Bäklin.

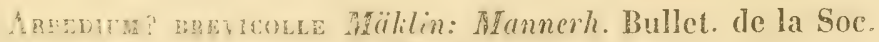
de illoscou. 1852. I. p. 320. 66.

Muribus indivinis, a D. F. Franticulacuser in parte inte-

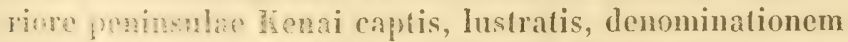

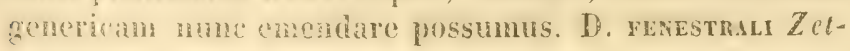


terst. haec species statura corporis simillima cst, sed

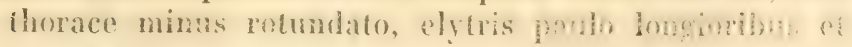
nomnihil fortius punctatis diversa. ("Mäklin.")

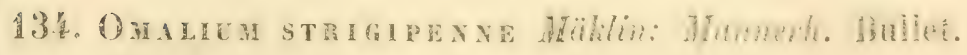
de la Soc. de Moscou. 1852. 1. p. 320.68.

Var. c. rufo-bumea, antemis pedibngur rufotestaceis, capite et ablomins ad partun nimbantilus.

Omaluas onscurrcole Motschulsky, in litteris.

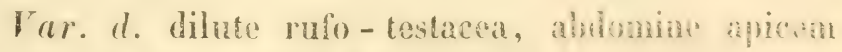
versus vix infuscato.

Omalum onifonas Motschulshy, in lilteris.

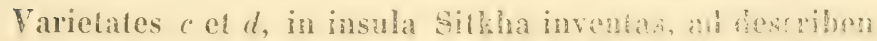

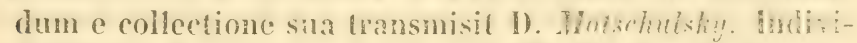
dua hujus species mulo majora 2 . Lin harturline in insula Kadjak cepit D. Holmberg.

Ab O. xano Grat. Erichs. cui simillima est hume spesta. prieter magnitudinem dindio aut introm thiplo majorem, inprimis thorace minus transverse theile distonguitur. ("Mälilin.")

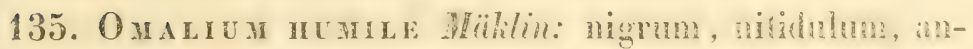
tennarum basi pedibusque rufo-tastaces, thonats picei lateribus elytrisque rufo-castanein, illo crome subtiliter punctato, dorso foreolis durhus vir emonicuis notato; his thorace plus quam dimidio longindbus, confertim subtiliter punctatis, subrumblosis, hngitudinaliter substrigulosis.

Longit. $1 \frac{1}{5}-1 \frac{1}{4}$ lin. Latit. $\frac{1}{5}$ fere $\frac{1}{2}$ lin.

In interiore parte peninsulae lienai prope if tschmmitan,

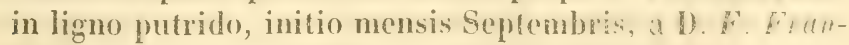
kenhaeuser rarissime inventum. 


\section{6}

$\Lambda$ praecedente magnitudine multo minore, punctura subtiliore et densiore et elytris brevioribus praecipue diversum. ("Mäklin.")

136. O imaidi fiavipene Motschulsky: nigrum, ore, antennarum basi (an totis?), elytris pedibusque rufotestaceis; thorace subquadrato, dense subtilius punctato; elytris thorace paulo plus quam duplo longioribus, dense et paulo profundius quam thorax punctatis, subrugulosis.

Longit. $1 \frac{1}{2}$ lin. Latit. $\frac{1}{2}$ lin.

Individuum unicum mutilatum, ut in insula Sitkha captum, ad describendum communicavit D. Motschulsky.

Quum Anthobum flavipenne Erichs. eadem sit species ac Axth. Lapponicum Mannerh. (Omalum Lapponicum Précis des Brachél. p. 52. 13.), denominationem speciei heic descriptae a Nob. Motschulsliy propositam conservatam esse voluimus ("Mäklin.")

137. Omalium planipenne Mälin: elongatum, depressum, nigrum, parum nitidum, elytris saturate nigropiceis, antennarum basi pedibusque rufo-testaceis; thorace obsolete canaliculato, dorso utrinque obsolete impresso.

Longit. $1 \frac{1}{3}$ lin. Latit. $\frac{1}{5}$ lin.

Habitat in insula Sitkha rarissime, ibique bis tantum a DD. Holmberg et $\boldsymbol{A}$. Collan inventum. In interiore parte peninsulae Kenai frequenter occurrit; D. F. Frankenhaeuser.

Ab O plaxo Payl. Erichs. antennis apicem versus paulo minus incrassatis, magnitudine paulo majore et elytris saturatioribus diversum mihi esse videtur. ("Maiklin.") 


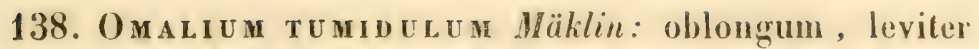
convexum, nitidum, subglabrum, capite, thorace elytrisque confertim punctalis; thorace latitudine plus quam duplo breviore, in medio sat fortiter dilatato, margine laterali profunde angulatim trirepando, puncto submarginali basin versus utrinque profunde irnpresso; elytris thorace duplo fere longioribus.

Longit. $1 \frac{1}{4}$ lin. Latit. vix ultra $\frac{1}{3}$ lin.

In insulis Sitkha et Afognak rarissime obvium; I. IIolmberg.

Quatuor individua, quae vidimus, colore inter se differunt: unum nigrum est, antennarum basi, elytris, pedibus anoque brunneo-rufis; alterum nigrum, thorace, elytris anoque piceis, antennarum basi pedibusque rufis; tertium rufo-brunneum, antennis apicem versus, capite elytrisque nigris; quartum brunneo-rufum, capite piceo. ("Mäklin. .)

139. A т то в гем в gulosum Mälilin: nigrum, parum nitidum, elytris sordide — antennis basin versus pedibusque dilutius testaceis; thorace leviter transverso, parcius obsoletiusque punctalo; elytris thorace plus quam duplo longioribus, crebre punctatis, transversim leviter rugulosis; maris apice rotundatis; feminae breviter acuminatis.

Longit. vix ultra 1 lin. Latit. $\frac{1}{2}$ lin.

IIabitat in insula Sitkha; D. Ilolmberg.

A. cuteipenvi Erichs aftine, sed thorace paulo longiore, an gustiore et evidentius punctato, elytrisque nomihil bre vioribus, minus subtiliter punctatis ef evidentius transversim rugulosis diversum. ("Mälilin:")

140. Micropeplus cat i coldis Mäklin: piceus, anten- 


\section{8}

nis, thoracis lateribus, pedibus anoque rufis; thorace latitudine plus duplo breviore et elytris quarta fere parte latiore; elytris apice depressis, dorso tricostatis, interstitiis minus crebre fortiter punctatis.

Var. b. rufo-brumea, capite nigro, medio thoracis parum infuseato.

Longit. vix ultra 1 lin. Latit. fere $\frac{1}{9}$ lin.

In interiore parte peninsulae Kenai a D. F Frankenhateuser sat frequenter captus; Var. $b$. rarissima.

M. Starnumond Marsham, Erichs. mihi inviso, statura corporis angustiore similis, sed thorace adhuc latiore et punctura elytrorum minus crebra diversus. ("Mäklin.")

1. 1. Micropeples costrpencis Mälilin: nigger, nitidus, thoracis lateribus pedibusque picescentibus; elytris dorso tricostatis, interstitiis laevibus.

Longit. fere $\frac{3}{4}$ lin. Latit. $\frac{7}{3}$ lin.

Etiam a D. F. Frankenhacuser in interiore parte peninsulae Kenai, sed rarissime inventus.

MI. trssenula Curis, Eriehs. dimidio major, elytris longioribus, colore antennarum pedumque diversus; a NI. BRUxneo Hälilin. (Mannerh. Bullet. de la Soc. de Moscou. 1852. 1. p. 326. 81.), cui magnitudine fere aequalis est, statura multo angustiore, nitore et colore totius corporis facillime distinguitur. ("Mäklin.")

\section{TRICHOP'TER VGIA.}

142. Ptrutu Goldani IIälin: oblongum, nigrum, opacum, crebre evidentiusque punctatum, dense subtiliter pubescens; thorace transverso, lateribus parum ro- 
Indato; elytris ablomine haud brevioribus, eorum apice pedibusque testaceis.

Longit. fere $\frac{1}{3}$ lin. Latit. $\frac{1}{6}$ lin.

IIabitat in insula Sithha, ubi individum unicum captum est a D. A. Collan.

P. Kunzei Heer quodammodo simile et afiine, sed multo majus, capite ratione magnitudinis minore el elytris longioribus imprimis diversum. ("Mällin.")

\section{A N I S O T O M I A.}

143. HYDNOBIS PUNCTOSTRA TUS firby: hreviter ovalus, convexus, obseure rufo-castancus, antennarum clava infuscata; capite thoraceque profunde remote punctatis, hoc transverso, undique marginato, margine pallidiore; elytris densissime profunde striato-punctalis, interstitiis laevibus, sutura discoque longitudinaliter obsolete infuscatis.

Longit. 1 lin. Latit. $\frac{7}{13}$ lin.

Leiones punctostniatus Kirby in Richardson, Fama Bor. Amer. IV. p. 110. 156.

In cortice arborum, initio Septembris, ad rivulos 11. Tschunuktnu peninsulae Kenai, a D. F. Frankenlueuser rarius inventus.

1'\%. Anisotoma late: ovata, valde convexa, rufoferruginea, oculis nigris; antennis articulo octavo minutissimo, clava elongata infuscata; thorace transverso, crebre punctato, lateribus modice rotundato valde declivi, angulis posticis obtusis; elytris profunde striato-punctatis, interstitiis tertio, quinto, septimo 
et nono punctis valde remotis seriatis; tibiis posticis rectis.

Longit. $1 \frac{3}{4}$ lin. Latit. $\frac{\pi}{4}$ lin.

Ilabitat in insula Sitkha; D. Ferd. Sallberg.

145. Anisotoma curvata: ovalis, convexa, rufo-castanea, nitida, pedibus dilutioribus; thorace transverso, remote punctulato, angulis posticis subrectis; elytris profunde striato - punctatis, stria tertia extrorsum flexa, interstitiis subtiliter punctatis, secundo medio latiore, tertio, quinto, septimo et nono punclis magnis remotis seriatis; tibiis posticis rectis.

Longit. $1 \frac{1}{3}$ lin. Latit. $\frac{3}{4}$ lin.

Ad rivulos fl. Tschunuktnu peninsulae Kenai, mense Julio, in volatu a D. F. Frankenhaeuser rarius capta.

146. Agathidium effluens: rotundato - globosum, postice attenuato - acuminatum, nigro - piceum; thorace brevi transverso, subtiliter punctulato, lateribus rufescente; elytris sat crebre punctatis, apice late rufoferrugineis, stria suturali postice impressa; pedibus rufo-testaceis.

Var. b. rufo - ferruginea vel ferrugineo - testacea, elytrorum apice semper pallidiore.

$$
\text { Longit. } 1-1 \frac{1}{4} \text { lin. Latit. } \frac{0}{3}-\frac{5}{6} \text { lin. }
$$

Sub cortice arborum ad rivulos fl. Tschunuktmu peninsulae Kenai, exitu Augusti, sat frequenter obvium; D. F. Frankenhaeuser.

Ab A. concinvo Mannerh. (Bullet. de la Soc. de Moscou. 1852. p. 370. 175.) differt elytris postice attenuato - acuminatis, apice rufo-ferrugineis et punctura evidentiore. 
147. Agathidum mandideatum: globosum, nigro piceum, nitidum, subtilissime creberrime punctulatum; mandibulis validissimis exsertis; antennis basi rufo-ferrugineis, clava magna pubescente; thorace rufo-piceo, limbo omni anguste pallidiore; elytrorum stria suturali posterius valde impressa.

Var. b. labro, frontis macula media quadrangula, antennarum basi et articulo ultimo, thorace, elytris pedibusque rufo - ferrugineis, thorace macula discoidali biloba elytrorumque angulis anticis infuscatis.

Longit. $1 \frac{1}{4}-1 \frac{1}{5}$ lin. Latit. $\frac{5}{6}-$ lin.

Habitat in insula Sitkha; D. Ferd. Sahlberg.

148. Grambus oblongulus: oblongo-ovatus, ferrugineus, subtilissime crebre punctulatus, pube brevi densa cinerea obductus; capite thoraceque nonnihil infuscatis; elytris postice attenuatis, apice subtruncatis pallidioribus.

Var. b. ferrugineo-testacea, elytrorum apice nonnihil pallidiore.

Longit. $\frac{2}{5}-\frac{3}{4}$ lin. Latit. $\frac{5}{1 \frac{3}{3}}-\frac{1}{2}$ lin.

Ad rivulos fl. Tschunuktnu peninsulae Kienai, exitu Augusti, sub cortice arboreo in terra jacente, a D. F. Frankenhaeuser sat frequenter lectus.

\section{N I T I D U L A R I A.}

149. Epuraen placida Mälilin: ovata, leviter convexa, sordide testacea, antennarum clava thoracisque medio infuscatis; thorace antice leviter emarginato; elytris apice singulatim rotundatis. 
Var. b. tota infuscata, antennis basin versus, capite, thoracis lateribus late et elytrorum margine anguste testaceis.

Longit. $1-1 \frac{1}{5}$ lin. Latit. $\frac{1}{8}-$ fere $\frac{8}{5}$ lin.

In truncis arborum, versus finem mensis Julii, ad 1 . Tschunuktnu peninsulae Kenai a D. F. Franticnhaeuser rarius capta.

E. Convexiuscula Mannerh. (Bullet. de la Soc. de Moscou. 1843. p. 253. 176.) minor, thorax antice latius, sed multo minus profunde emarginatus. E. tenmivat Munuerh. statura corporis simillima, sed plerumque paulo minor, punctura crebriore et paulo fortiore ut et colore diversa. ("Mälilin.")

150. Epuraea playulata Eschscholtz: Mannerí. Bullet. de la Soc. de Moscou. 1852. 1. p. 337. 105.

Var. c. ferruginea, thorace macula obsoleta elytrisque fascia media longitudinali fuscis.

Var. d. fusco-nigra, capite, thorace, elytrorum limbo, antennarum basi pedibusque ferrugineis; thorace macula media ut in Var. $c$.

Var. c. in insula Sitkha inventa est a D. A. Collen; Var. d. in insula Kadjak a D. Holmberg et in interiore parte peninsulae Kenai, simul cum individuis genuinis, a l). F. Frankenhaeuser. ("Mälilin.")

151. Epuraen nigra Mälitin: oblonga, depressa, creberrime punctata, nigra vel nigro - picea, ore geniculisque dilutius piceis; thorace antice leviter sed late emarginato, lateribus parum rotundato, margine subrepando; elytris apice rotundato - truncatis.

Longit. $1 \frac{1}{4}-1 \frac{1}{3}$ lin. Latit. $\frac{1}{2}$ lin. 


\section{3}

In interiore parte peninsulac lienai a 1). F. Frankenhacuser sat frequenter lecta.

E. moRerlae 2 clterst. Erichs. simillima, sed staiura corporis panlulum angustiore, thorace antice minus profunde emarginato, lateribus levius rotundatis ut et colore diversa $\left({ }^{\star}\right) \cdot($ Mäklin.")

152. EPCRAEA FLA VouAcLATA Füllin: oblonga, subparallela, depressiuscula, nigra, antennarum basi, thoracis lateribus, elytrorum limbo laterali et apicali maculisque duabus in singulo, una prope scutellum, allera pone medium, flavo-testaceis; thorace antice late emarginato; elytris apice oblique truncatis.

Longit. $1 \frac{2}{3}$ lin. Latit. $\frac{5}{4}$ lin.

Ad castellum Nicolajevsk peninsulae Kenai, sub cortice arboris, a D. Holmberg semel capta. ("Mäklin.")

153. WPURAEA LIXEAIS Mulilin: elongata, linearis, ferruginea, subtilissime punctulata, antennarum clava, thoracis disco, elytrorum sutura basin versus latius indeterminatim fasciaque postice obliqua, interdum dilatata apicem totum late occupante, nigro-fuscis; thorace apice late leviter emarginato; elytris apice singulatim obtuse rotundatis, subtruncatis.

Longit. $1 \frac{1}{3}$ lin. Latit. fere $\frac{1}{2}$ lin.

Individua nomulla in parte interiore peninsulae Kenai a

D. F. Frankenhaeuser inventa sunt.

Species haec, punctura omnium subtilissima insignis, statura corporis quandam cum E. tnuncatella Mannerh.

(") Ad Ajan Sihiriae orientalis noram, hisque quam maxime affinem, invenerunt speciem (EPrrae. pICEa Mäklin. DD. Pippingsköld et Collan. Differt vero hace species ab utroque angulis thoracis anticis multo acutioribus. (《IIäklin.») 
(Bullet. de la Soc. de Moscou. 1846. II. p. 514. 12.) praebet similitudinem, sed thorace paulo longiore, apice leviter emarginato, lateribus adhuc magis rectis, magnitudine paulo minore et imprimis punctura totius corporis diversa. ("Mälilin. ")

154. Epuraea infuscata Mälin: ovata, leviter convexa, ferrugineo - testacea, antennarum clava, medio thoracis plerumque, pectore elytrisque apicem versus oblique, infuscatis; thorace antice emarginato; elytris apice singulatim obtuse rotundatis, subtruncatis.

Nitidula nufa Say, Journ. of the Acad. of Nat. Scienc. of Philadelph. V. p. 180. 4?

Var. b. medio thoracis, elytris exterius oblique, pectore abdomineque fusco nigris.

Longit. $1 \frac{1}{2}-1 \frac{2}{3}$ lin. Latit. $\frac{4}{5}$ lin.

Habitat in truncis arborum ad fl. Tschunuktnu peninsulae Kenai versus finem mensis Julii, ubi a D. F. Frankenhaeuser copiose lecta. ("Mäklin.")

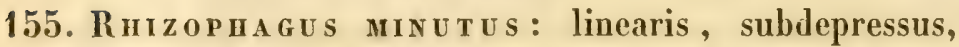
nigro-piceus, nitidus, thorace latitudine vix longiore, remote oblongo-punctato, lateribus nonnihil rotundato; elytris piceo - castaneis, striato - punctatis, apice obscurioribus truncatis; ore, antennis pedibusque rufis.

Longit. 1 lin. Latit. $\frac{1}{3}$ lin.

Ad rivulum fl. 'Tschunuktnu peninsulae Kenai, initio Julii, sub cortice Populi, specimen unicum invinit D F. Frankenhaeuser.

Ri. parvelo Payk. minor, brevior, fortius punctatus coloreque ab illo dissimilis. 


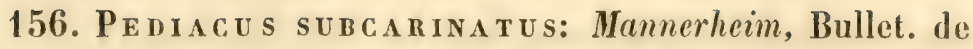
la Soc. de Moscou. 1852. I. p. 363. 163.

Var. b. tota rufo-castanea vel rufo-ferruginea, oculis tantum nigris.

Longit. $1 \frac{1}{2}-1 \frac{9}{3}$ lin. Latit. $\frac{5}{12}-\frac{1}{2}$ lin.

Sub cortice populi et pini, ad rivulos fl. Tschunuktnu peninsulae Kenai, mense Julio, una cum Var. a. a D. $F$. Frankenhaeuser copiose lecta.

$\Lambda$ P. Fusco Erichs. thorace angustiore, elytris longioribus et carinula thoracis plus minusve distincta diversus.

157. Dendrophagus Americanus: lineari - elongatus, depressus, nitidus, capite, thorace pectoreque nigro piceis, ore, antennis abdomineque castaneo - ferrugineis, elytrorum margine basali toto et laterali ultra medium dilutius ferrugineis; vertice lineis duabus, parallelis, angustis impresso; thorace oblongo, crebre punctato, lateribus integro, dorso longitudinaliter biimpresso; elytris crebre et profunde crenato - striatis.

Longit. 3 lin. Latit. $\frac{5}{6}$ lin.

Specimen unicum ad rivulum fl. Tschunuktnu peninsulae Kenai, medio Septembris, volitans cepit D. F. Frankenhacuser.

Inter D). crenatum Schönh. et D). Cygnaei Mannerh. (Bullet. de la Soc. de Moscou. 1846. p. 515. 14.) medius, illi colore similis, capite vero lineis duabus insculptis (haud longitudinaliter biimpresso), thorace longiore, angustiore, lateribus vix sinuato, elytris brevioribus striisque eorum crebrius punctatis diversus: ab utrisque elytris brevioribus et a D. Crgnat praeterea antennis validioribus capitisque sulcis simplicibus, in occipite haud bifidis distinctus. 


\section{6}

\section{R Y P T O P A GA.}

158. Axtmeropingus suturatis fïälin: oblongus, niger, parce subtilissime pubescens, elytrorum piceorum margine omni, apicali latius, suturali antrorsum abbreviatim, antennis pedibusque rufo - testaceis; thoracis angulis posterioribus subrectis.

Longit. $1 \frac{1}{2}-1 \frac{2}{3}$ lin. Latit. $\frac{\pi}{4}$ lin.

A D. F. Frankenhaeuser in interiore parte peninsulae Kenai rarissime captus. ("Mälilin.")

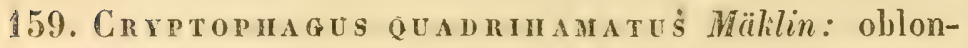
gus, leviter converus, dense subtiliterque punctatus, niger, subnitidus, pube depressa grisea vestitus, antennis excepta clava picea, macula subhumerali elytrorum pedibusque ferrugineo - rufis; thorace leviter transverso, obsoletius tuberculato, lateribus acute bidentatis, dente etiam posteriore subhamato.

Longit. vix altra 1 lin. Latit. $\frac{1}{3}$ lin.

In interiore parte peninsulae Kenai a D. F. Frankenhaeuser detectus. ("Mäklin.".)

160. CRy topha gus i I bentatus Mälitin: oblongo-ovatus, leviter converus, dense subtilius punctatus, ferrugineus, pube brevi depressa vestitus; thorace transverso, lateribus subbidentatis, dente anteriore parum prominulo obtuso, posteriore ante medium sito obsoleto, carinula ante scutellum nulla.

Longit. $\frac{5}{6}$ lin. Latit. $\frac{7}{5}$ lin.

In societate cum specie proxime insequente a D. F. Franlienhaeuser rarissime captus.

Vestigium tuberculorum thoracis punctis tantum laeviga tis indicatum est. ("Mälilin.") 
161. CR Y P T OP I A G U P U C T A T Is I u u Mällin: oblongoovatus, leviter convexus, confertim punctatus, fuscoferrugineus, pube brevi depressa dense vestitus; thorace transverso, lateribus bidentatis, dente anteriore parum prominulo, obtuso, posteriore in medio sito, carinula ante scutellum nulla.

Longit. $1 \frac{1}{6}$ lin. Latit. fere $\frac{1}{2}$ lin.

In interiore parte peninsulae Kenai a D. F. Frankenhacuser rarissime inventus. ("Mäklin. w)

162. Paramecosoma serrata Gyllenhal: nigra aut nigro-picea, pubescens, antennis pedibusque ferrugineis; thorace lateribus serrato; elytris plerumque dilutius rufo-piceis.

Longit. 1 lin. Latit. $\frac{1}{3}$ lin.

Erichson, Insekt. Deutschl. p. 374. 5. - Sturm, Fauna.

XVIII. p. 78. 5. - Redtenbacher, Fauna Austr. p. 193. -

Gaubil, Cat. p. 69. 5. - Bach, Käferfauna. p. 2כ3. 3. Cat. Col. Europ. 1852. p. 37.

Cryptophagus serratus Gyllenhal, Ins. Suec. I. p. 171. 7. Sahlberg, Ins. Fenn. I. p. 56. 7. - Stephens, Illustr. Mandib. III. 78. Manual p. 137 1106. - Sturm, Fauna XVI. p. 109. 24. Tab. CCCXIX. fig. B

Plura individua hujus speciei, Fennicis et Lapponicis omni modo similia, in interiore parte peninsulae Kenai legit D. F. Frankenhaeuser. ("Mäklin.")

163. A tomaria ferruginea Sahlberg: oblonga, subdepressa, ferrugineo-testacea, pubescens; antennis approximatis, harum clava biarticulata.

Longit. 1 lin. Latit. parum ultra $\frac{t}{t}$ lin. 


\section{8}

Erichson, Insekt. Deutschl. p. 377. 1. - Redtenbacher, Fauna Austr. p. 194. - Gaubil, Cat. p. 69. 1. - Bach, Käferfauna. p. 254. 1. - Cat. Col. Europ. 1852. p. 37.

Cryptophagus renrugineus Salilberg, Ins. Fenn. I. p. 58.11.Gyllenhal, Ins. Suec. IV. p. 290. 12-13.

Specimina quaedam in interiore parte peninsulae Konai capta sunt a D. F. Frankenhaeuser. ("Mäklin ")

164. A tomaria vespentina Mäklin: oblonga, depressa, nigra, nitida, minus dense punctata, subtiliter cinereopubescens, antennis praeter clavam infuscatam, pedibus ely trisque rufo - ferrugineis, his in medio plerumque plus minusve nigricantibus; thorace elytris parum angustiore; antennis approximatis.

Longit. $\frac{4}{5}$ lin. Latit. parum ultra $\frac{1}{4}$ lin.

Individua pauca in interiore parte peninsulae Kenai, sero vespere volitantia, cepit D. F. Frankenhaeuser. ("Mäklin.")

165. A tomari plandata Mäklin: oblonga, depressa, testaceo-fusca, nitida, subtiliter dense punctata, pube cinerea subtili vestita, antennis, pedibus elytrisque rufo-testaceis; thorace elytris nonnihil angustiore; antennis approximatis.

Longit. $\frac{4}{5}$ lin. Latit. parum ultra $\frac{1}{4}$ lin.

In insula Sitkha a D. Collan semel tantum inventa.

$\Lambda$ praecedente punctura crebriore et multo subtiliore, statura totius corporis adbuc magis depressa ut et colore facile distinguitur. ("Mälilin.,)

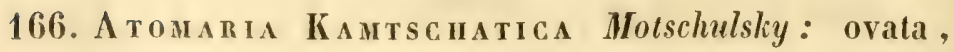
convexa, nigra, nitida, subtiliter dense punctata, pube 
grisea subtili vestita, antennis, humeris apiceque elytrorum late et pedibus rufo-testaceis; thorace in medio dilatato-rotundato; antennis distantibus.

Var. b. nigro-picea, elytris dilutius fusco-piceis, aut tota fusco-picea, antennis pedibusque ut in specie genuina.

Var. c. tota rufo-testacea.

Longit. $\frac{2}{3}-\frac{3}{4}$ lin. Latit. $\frac{1}{3}$ lin.

Motschulsky, Bullet. de la Soc. de Moscou. 1845? p. 362.40.

In insula Kadjak a D. Molmberg lecta; occurrit etiam in Sibiria orientali. I). Pippingskëld mihi individuum ad portum $\Lambda$ jan captum transmisit et $\mathbf{D}$. Collan nomulla in peninsula Kamschatka et ad $\Lambda$ jan invenit. Var. $c$. tantum ex hac localitate Sibiriae orientalis a D. Collan allata. ("Mäklin. ")

\section{A T H R I D I A.}

167. Corticaria canaliculata: elongata, nigro picea, subnitida; thorace orbiculato, profunde et crebre punctato, lateribus denticulato, medio canaliculato, fovea postica impresso; elytris oblongis, convexis, profunde punctato - striatis, interstitiis valde rugosis; antennis pedibusque rufo - piceis.

Longit. 1 lin. Latit. $\frac{1}{2}$ lin.

Habitat ad fl. Tschunuktnu peninsulae Kenai ibique exitu. Junii et initio Julii, in volatu a D. F. Frankinhaeuser. saepius capta.

168. Corticaria orbicollis: elongata, nigro-picea, parce griseo-pubescens; thorace orbiculato, profun- 
de et crebre punctato, lateribus crenulato, fovea magna postice impresso; elytris oblongis convexis, profunde punctato - striatis, interstitiis vix rugosis, alternis ante medium elevatioribus, humeris rufescentibus; antennarum basi pedibusque rufis.

Longit. $\frac{3}{4}$ lin. Latit. $\frac{5}{1^{2}}$ lin.

Ad castellum Nicolajevsk peninsulae Kenai, exitu Junii, sub cortice arboris nuper caesae, semel legit D. F. Fran. kenhaeuser.

169. Corticaria deleta: elongata, obscure rufo-castanea, parce griseo-pubescens; thorace longitudine latiore, crebre punctato, lateribus rotundato, mutico, fovea postica impresso; elytris oblongis convexis, creberrime punctulatis, striatis, striis pone medium evanescentibus; antemnis pedibusque rufis.

Var. b. rufo-ferruginea, capite infuscato.

Longit. $\frac{2}{3}$ lin. Latit. $\frac{5}{1 \frac{8}{8}}$ lin.

Circa fl. Tschunuktnu peninsulae Kenai, exitu Junii et ini-

tio Julii, volitans saepius occurrit; D. F, Frankenhaeuser.

Var. $b$. etiam ad ostia fl. Kaktnu ejusdem peninsulae et in insula Sitkha a D. Holmberg rarius lecta.

170. Corticaria exigua : oblonga, nigro-picea, parce griseo-pubescens; thorace longitudine latiore, creberrime punctulato, lateribus rotundatis, postice obsoletissime crenatis, fovea antescutellari impressa; elytris oblongis convexis, creberrime punctulatis, profunde punctato - striatis, striis pone medium evanescentibus; antennis pedibusque rufis, femoribus nonnihil infuscatis.

Longit. $\frac{1}{2}$ lin. Latit. $\frac{1}{4}$ lin. 
In volatu frequentius capta, exitu Junii et initio Julii, ad 11. Tschunuktnu peninsulae Kenai, D. F. Frantientaeuser.

171. Laturidú Cinnamopterus : oblongo - ovatus, rufo-piceus, antennis, elytris pedibusque rufo-ferrugineis; capite rugoso, vertice triangulariter inpresso; thorace quadrato, rugoso, antrorsum vix latiore, angulis anticis rotundatis, lateribus subrectis, dorso depresso, in medio carinulis duabus antrorsum divergentibus obsoletis; elytris oblongo - ovatis, ampliatis, apice attenuatis, profunde striato - punctatis, striis posterius evanescentibus, interstitio tertio ad basin valde elevato, humeris parum prominulis.

Longit. $\frac{5}{6}$ lin. Latit. $\frac{5}{18}$ lin.

Iisdem locis, eodem tempore et quoque in volatu, uti praecedens species, a D. F. Frankenhaeuser saepius inventus.

172. LATIRIDIUS FULVIPENNIS: breviter ovatus, fuscocastaneus, antennis, elytris pedilousque laete fulvorufescentibus; capite rugoso, vertice triangulariter impresso; thorace subquadrato, rugoso, antrorsum nounihil dilatato, angulis anticis rotundatis, intra illos utrinque foveolato, lateribus leviter emarginato, dorso depresso, basi transversim trifoveolato, carinulis duabus in medio parallelis; elytris breviter ovatis, valde ampliatis, margine explanatis, profunde striato - punctatis, humeris gibbosis, apice attenuatis, subacuminatis.

Longit. $\frac{3}{4}$ lin. Latit. $\frac{1}{2}$ lin.

In peninsula Kenai ad castellum Nicolajevsk, exilu Junii, sub ligno in terra jacente, specimen unicum a $\mathrm{D}$. Hohmberg lectum. 


\section{2}

173. Laturidus incisus Mannerheim: elongatus, angustus, fusco-castaneus, opacus; thorace sub-lyrato, pone medium constricto, dorso obsolete bicostato, costis anterius divergentibus; elytris profunde striatopunctatis, interstitiis latioribus, argute elevatis.

Longit. $\frac{3}{4}$ lin. Latit. $\frac{2}{3}$ lin.

Mannerheim, in Germar Zeitschrift. V. p. 80. 5. - Redtenbacher, Fauna Austr. p. 780. - Gaubil, Cat. p. 71. 10. Bach, Käferfauna. I. p. 267. 5. - Cat. Col. Europ. 18 ¿2. p. 96.

Ad fl. Tschunuktnu peninsulae Kenai, exitu Junii et initio Julii, in volatu non infrequenter obvius; D. F. Frankenhaeuser.

174. Latiridius strangulatus: oblongo - ovatus, rufo-ferrugineus, nitidus, glaber; capite canaliculato; thorace latitudine longiore, ruguloso, medio canaliculato, pone medium lateribus utrinque profunde inciso, intra basin transversim impresso; elytris breviter ovatis, profunde striato - punctatis.

Longit. $\frac{2}{3}$ lin. Latit. $\frac{2}{3}$ lin.

Cum praecedente, etiam in volatu, a D. F. Frankenhaeuser aliquoties captus.

175. Latiridius minutus Linné: breviter ovatus, nigropiceus, antennis pedibusque rufo-testaceis; thorace subquadrato, angulis anticis rotundato-ampliatis, dorso subdepresso, crebre rugoso-punctato, in medio longitudinaliter foveolis duabus oblongis et basi transversim impresso; elytris subconvexis, nitidis, profunde striato-punctatis.

$V a r . b$. rufo-ferruginea.

Longit. $\frac{3}{4}-1$ lin. Latit. $\frac{3}{8}-\frac{1}{2}$ lin. 
Mannerheim, in Germar Zeitschrift. V. p. 96. 34. (unde synonymiam repetas) - Redtenbacher, Fauna Austr. p. 206. - Gaubil, Cat. p. 71. 2\%. - Bach, Käferfauna. I. p. 268. 10. - Cat. Col. Europ. 1852. p. 96.

In peninsula Kenai, ad fl. Tschunuktnu, exitu Junii et initio Julii, volitans a D. F. Frankenhaeuser frequenter, ad castellum Nicolajevsk vero, eodem tempore, sub lignis caesis, a D. Holmberg rarius lectus.

176. LA TII I D US PARALLE OCOLLIS Mannerheim: breviter ovatus, nigro-piceus, glaber, antennis pedibusque rufo-testaceis; thorace quadrato, lateribus parallelis rectis, angulis omnibus rotundatis, dorso depresso, rugoso, obsolete canaliculato, basi transversim impresso; elytris ovatis, profunde punctato-striatis, interstitio secundo lato, ceteris angustis, alternis subcostatis.

Longit. $\frac{8}{5}$ lin. Latit. $\frac{1}{\frac{1}{8}}$ lin.

Mannerheim, in Germar Zeitschrift. V. p. 101. 40. Gaubil, Cat. p. 72. 31. - Cat. Col. Europ. 1852. p. 96.

Ad 1. Tschunuktnu peninsulae Kenai, simul cum praecedente in volatu a D. F. Frankenhaeuser saepius captus.

177. LATHRIDIUS CURTULUS: breviusculus, rufo-piceus, opacus; capite crebre rugoso-punctato; thorace cordato, valde rugoso, in medio bicarinato, carinulis antrorsum nonnihil divergentibus; elytris posterius dilatatis, fusco-piceis, profunde striato-punctatis, interstitiis tribus elevatis, costatis.

Longit. $\frac{7}{1^{7}}$ lin. Latit. $\frac{1}{3}$ lin.

IIabitat in insula Sitkha; D. Ferd. Sahlbery. 


\section{E R M E S T I N A:}

178. Attagenus angularis: oblongus, convexus, subcylindricus, nigro - piceus, parce griseo - pubescens; antennis rufo - ferrugineis, clava ovata; thorace brevi, transverso, crebre confertim punctulato, antrorsum valde angustato, lateribus marginato, margine reflexo, basi profunde bisinuato, angulis posticis pubescentia condensata albidis; elytris subtiliter creberrime rugoso - punctatis.

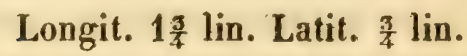

Sub cortice Populi, ad rivulum fl. Tschunuktnu peninsulae Kenai, mense Augusto, a D. F. Frankenhaeuser semel inventus.

\section{B Y R R H I A.}

179. Sychlypta Setulosa: breviter ovata, convexa, nigra, fusco - squamulosa, setis erectis clavatis nigris longioribus adspersa; thorace lateribus antice leviter compresso; elytris striatis, stria suturali tantum postice profundiore.

Longit. 1 lin. Latit. $\frac{3}{4}$ lin.

In regionibus interioribus peninsulae Kenai individuum unicum a D. F. Frankenhaeuser lectum.

S. setigera Illig. minor, undique fusco - squamulosa, haud albido-variegata, et elytrorum stria suturali postice profundius, reliquis aequaliter impressis distincta.

180. Byrrius cyclophorus Kirby: breviter ovalis , nigro-piceus, subtilissime coriaceus, cinereo-pubescens; antennis rufescentibus, articulo primo et ulti- 
mis infuscatis; elytris vittis duabus, nonnihil interruptis, nigris, dorso macula media communi, subcirculari, antice emarginata, e squamulis fusco-aeneis.

Longit. $3 \frac{1}{4}$ lin. Latit. 2 lin.

Richardson, Fauna Bor. Amer. IV. p. 117. 67. - Steffahny, Monogr. in Germar Zeitschrift. IV. p. 24. 18.

Ad fl. Tschunuktnu peninsulae Kenai, medio Junii, in gramine a D. F. Frankenhaeuser semel captus.

181. Simplocaria metallica Sturm: oblonga, nigrosubaenea, nitida, griseo-pubescens; thorace subtiliter minus crebre punctato; elytris continue striatis, striis interioribus profundioribus, secunda pone medium, tertia longe ante apicem cum suturali conniventibus, interstitiis obsolete punctulatis, pube grisea maculatim vestitis; antennis pedibusque rufescentibus, femoribus infuscatis.

Longit. $1 \frac{1}{8}-1 \frac{3}{4}$ lin. Latit. $\frac{3}{4}-1$ lin.

Erichson, Deutschl. Ins. p. 494. 2. - Gaubil, Cat. p. 75. 2.Cat. Col. Europ. 1852. p. 39.

Simplocaria picipes Stephens Illustr. Mandib. III. p. 140. Manual p. 14T. 1184. - Steffahny, Monogr. in Germar Zeischrift. IV. p. 38. 2. - Redtenbacher, Fauna Austr. p. 226.

Brrauvs metallicus Sturm, Fauna. II. p. 111. 18. Tab. XXXIV. fig. 6. B. C. - Duftschmid, Fauna Austr. III. p. 19.19.

Byninus picipes Gyllenhal, Ins. Suec. I. p. 200. 8. - Sahlberg, Ins. Fenn. I. p. 66. 6. - Zetterstedt, Fauna Lappon. I. p. 133. 7. Ins Lappon. p. 93. 8.

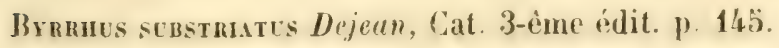


Habitat ad rivulos fl. Tschunuktnu peninsulae Kenai, mense Augusto, in gramine frequens; D. F. Frankenhaeuser.

Quamvis pedes obscuriores, in ceteris cum individuis nostris omnino congruit, quare ut eadem species consideranda.

\section{H E T E R O C E R A.}

182. Heterocerus tristis Motschulsky: oblongus, fuscus, subtiliter creberrime punctulatus, pubescens, capite thoraceque longius tomentosis; hoc brevissimo, transverso, elytrorum latitudine, angulis posticis angustissime marginatis; elytris lineis tribus minutis parallelis ad basin, lituris duabus in medio, quarum interiore obliqua, exteriore recta, posticeque duabus obliquis, quarum exteriore posterius collocata, atris, his lituris fasciis flexuosis duabus e pube grisea vario modo limitatis; tarsis tenuissimis, pallide ferrugineis.

Longit. $1 \frac{3}{4}$ lin. Latit. $\frac{3}{4}$ lin.

IIabitat in insula Sitkha, sec. D. Motschulsky, qui typum hoc nomine insignitum e museo suo ad describendum mihi benevole tradidit.

\section{S C A R B A I N A.}

183. Aprodius ursinus Eschscholtz: oblongus, valde convexus, niger nitidus, pedibus plus minusve piceis; clypeo leviter emarginato, vertice trituberculato; thorace longitudine sesqui latiore, crebre punctulato, basi leviter bisinuato, ibique et lateribus tenue marginato, his fere linearibus, vel antrorsum nonnihil angustatis et ante basin leviter subsinuatis, angulis anti- 
cis rufescentibus; elytris pone medium parum dilatatis, crenato - striatis, interstitiis laevibus.

Longit. 3 lin. Latit. $1 \frac{1}{3}$ lin.

Dejean, Cat. 3-ème édit. p. 160. - Motschulsky, Bullet de la Soc. de Moscou. 1845. II. p. 365. 47. Tab. VI. fig. 6.

$\Lambda d$ rivulos fl. Tschunuktnu peninsulae Kíenai, exitu Julii, in stercoratis a D. F. Frankenhaeuser rarius inventus. Specimina paullo majora sed ceterum omni puncto simillima, in peninsula Kamschatka capta, a D. Eschscholtz quondam obtinui.

184. Apuodus congregatus: oblongus, nigro-piceus nitidus; clypeo crebre granulato, emarginato, margine late ferrugineo; vertice trituberculato; thorace plus minusve remote punctulato, basi truncato lateribusque anguste marginato, his parum rotundatis, angulis anticis lateribusque rufo - ferrugineis, rubedine illa subtriloba; elytris striis modice exaratis, leviter crenatis, interstitiis laevibus; luteo - testaceis, lateribus late rufescentibus, scutello, utroque fasciis macularibus, una obliqua a basis medio ad medium suturae ducta, altera ab humero ad suturam pone medium continuata undulata, maculisque duabus intra apicem, brunneo-castaneis; pedibus rufo - testaceis.

Var. b. elytris rufo-testaceis, obsolete infuscatis; cetera ut in $a$.

Longit. 2-2을 lin. Latit. $\frac{5}{6}-1 \frac{1}{5}$ lin.

In quisquiliis domi vetustae et in stercore, ad sinum Woskresensk peninsulae Kenai, mense Majo, copiose legit D. Holmberg.

185. Aegiala exarata: oblonga, modice convexa, subcylindrica, supra piceo-castanea, subtus rufescens; 


\section{8}

clypeo apice emarginato, margine rufo, crebre ruguloso; vertice subtiliter punctulato; thorace transverso, lateribus rotundato, ciliato, angulis anticis vix productis, rotundatis, disco punctis paucis majoribus irregulariter sparsis, versus latera magis congestis; elytris striis profunde exaratis, in fundo leviter crenulatis, interstitiis laevibus, humeris dente minuto acutiusculo armatis.

Longit. $2 \frac{1}{5}$ lin. Latit. 1 lin.

Habitat in insula Sitkha, sec. D. Ménétriés, qui hujus speciei individuum e Museo Acad. Imp. Scient. Petrop. ad describendum mili benevole transmisit.

186. Aegialia cylindeica Eschscholtz.

Oxyomos cyundrices: Mannerh. Bullet. de la Soc. de Moscou. 1843. p. 262.194.

Var. $b$. thorace antice et lateribus, elytris basi et margine late pedibusque rufis.

Var. c. rufo-ferruginea, capite thoraceque infuscatis.

Var. d. rufo-ferruginea tota.

Longit. $1 \frac{3}{4}-2 \frac{1}{3}$ lin. Latit. $\frac{3}{4}-1 \frac{1}{4}$ lin.

Var. b. in insula Sitkha captam communicavit D. Ménctriés;

Var. $c$ et $d$. simul cum $a$ ad sinum Woskresensk peninsulae Kenai, initio Junii, sub lapidibus parce invenit D. F. Frankenhaeuser.

\section{B U P R E S T I A.}

187. Chrysoboturis trinervia Kirby: supra nigroaenea, subtus cupreo-aenea pilosa; nasu sinu obtusangulo, fronte crebre profunde punctata, plagis duabus 
minutis laevigatis subelevatis inter oculos; thorace apice emarginato, basi bisinuato, lateribus subrectis, vix emarginatis, in medio canaliculato, dorso lacunoso, foveis duabus in disco evidentioribus; elytris longitudinaliter trinerviis, nervis undulatis, exterioribus duabus irregularibus hine inde interruptis, vel cum nervis transversis connexis, spatiis pluribus punctatis subauratis; femoribus anticis incrassatis dentatis, tibiis quatuor anterioribus arcuatis.

Longit. 5 lin. Latit. $2 \frac{1}{5}$ lin.

Richardson, Fauna Bor. Amer. IV. p. 157. 210. Tab. II. fig. 9.

Cinysonoturis cicatricosa Motschulsky, Etudes entom. 1852.

p. 77.

Individuum, in insula Kadjak captum, e museo suo amice transmisit D. Motschulsky.

188. Melanopilla guttulata Gebler: obscure aenea, nitida, thorace transverso, basi bisinuato, crebre punctato - rugoso, disco utrinque saepe concentrice strigoso, vario modo et plus minusve foveis impressis inaequali, medio interdum obsolete canaliculato; elytris crebre granulatis, integerrimis, apice singulatim rotundatis, utroque guttis tribus luteis, prima ante medium a margine remotiore quam a sutura, tertia suturae adhuc propiore paullo ante apicem, et intermedia e prima et tertia aeque distante a sutura remotiore quam e margine.

Longit. 4 lin. Latit. $1 \frac{3}{4}$ lin.

Mannerheim, Bullet. de la Soc. de Moscou. 1837. VIII.

p. 69. 2. - Gebler, Bullet. de la Sor. de Moscou. 18'7. II.

p. 408. 3. 


\section{0}

Bupnestis guttulata Gebler in Ledebour Reise. II. 3. p. 80.12. Mém. de la Soc. de Moscou. VIII. p. 41. 5.

Bupnestis discopunctata Faldermann, Bullet. de la Soc. de Moscou. 1833. p. 47. Tab. II. fig. 5.

Buprestis (Trachypteris) Drummond Kirby in Richardson, Fauna Bor. Amer. IV. p. 159. 11. Tab. II. fig. 2. Var. b.

Apatuna discoponctata Laporte et Gory, Monogr. des Buprestides, Apatura. p. 3. Tab I. fig. 2.

Apatura Drummond Laporte el Gory, 1. c. p. 3. Tab. I. fig. 3. - Mannerheim, Bullet. de la Soc. de Moscou. 1843. p. 236.130.

Phanoops Gebler Dejean, Cat. 3-eme édit. p. 89.

Specimen ad fi. Kwichpakh in continenti Americes borealis captum e Museo Acad. Imp. Scient. Petrop. benevole communicavit D. Ménétriés. Occurrit in Sibiria e montibus Altaicis usque ad littora Maris Pacifici.

Quando statura plus minusve oblonga vel plus minusve lata, thorace jam magis, jam minus foveis impressis inaequali, elytrorumque impressionibus humeralibus plus minusve profundis variat, ex individuis Americanis speciem diversam constituere jure non liceat. Diagnosin emendatam ideoque elaboravi.

\section{E L A T E R I A.}

189. Atious triunduatus: nigro-piceus, dense cinereo-pubescens; thorace elongato, antrorsum parum angustato, crebre confertim punctato, angulis posticis elongatis divaricatis, impressione obliqua fere ad medium marginis ascendente a disco limitatis, posterius canaliculato, vitta utrinque a basi ad apicem continuata rufescente decorato; elytris punctato - striatis, interstitiis planis, subtiliter punctulatis, ferrugineo-ca- 
staneis, fasciis tribus transversis undulatis denudatis; genubus tarsisque piceo - testaceis.

Longit. $5 \frac{3}{4}$ lin. Latit. $1 \frac{1}{8}$ lin.

IIabitat in insula Sitkha, sec. D. Ménétriés, qui individuum in Mus. Acad. Imp. Scient Petrop. exstans ad describendum mihi amice transmisit.

A. undulato De Geer (trifasciato Gyllenh.) valdo aflinis, ita ut ejus varietatem esse facile credideris, sed accuratius examinatus jus speciei diversae tamen contendit; brevior nempe posteriusque minus angustatus, elytrorum striis minus profunde punctatis et interstitiis omnino planis, minime convexis imprimis distinctus, fascia denudata postica elytrorum etiam magis versus apicem remota.

190. Elater nigrines Paykull: ater, nitidus, pilosus; thorace lateribus antrorsum angustato, sat crebre punctato, angulis posticis acutiusculis argute carinatis, postice in medio longitudinaliter interdum impresso; elytris profunde punctato - striatis, interstitiis rugosis; antennis pedibusque piceo - testaceis.

Longit $3 \frac{1}{5}-4$ lin. Latit. $1-1 \frac{1}{3}$ lin.

Paykull, Fauna Suec. III. p. 39. 44. - Herbst in Fuessly Archiv. p. 144.33. - Gyllenhal, Ins. Suec. I. p. 421. 51.Schönherr, Syn. Ins. III. p. 296. 150. - Sahlberg, Ins. Fenn. I. p. 141. 42.-Eschscholtz in Thon Entom. Archiv. II. p. 33. - Gebler in Ledebour Reise II. 3. p. 83. 26. Castelnau, Hist. nat. des Ins. I. p. 245. 30. - Zetterstedt, Fauna Lappon. I. p. 25\%3. 31. Ins. Lappon. p. 150. 32. Redtenbacher, Fauna Austr. p. 787. - Gaubil, Cat. p. 110. 19.

Ampedus nigrinus Dejean, Cat. 3-ème édit. p. 105. Germar, Zeitschrift. V. p. 175. 35. - Gebler, Bullet. de la Soc. de Noscou. 1817. II. p. 419. 3. - Cat. Col. Europ. 1832. p 48. 
Aupedes nigrinus Bach, Käfer Fauna 3. p. 40.14.

Elater pilosulus Herbst, Col. X. p. 79. 76. Tab. ClXIV. fig. 2.

In peninsula Kenai ad sinum Woskresensk a D. Holmberg et in ejusdem peninsulae regionibus interioribus a $\mathbf{D}$. F. Frankenhaeuser captus.

Ab Ec. Canbonicolone Eschsch. (Mannerh. Bullet. de la Soc. de Moscou. 1843. p. 240. 142.) statura latiore, breviore, thorace fortius et crebrius punctato, elytrorum striis profundius punctatis, interstitiis fortius rugosis et antennis pedibusque dilutioribus distinguendus.

191. CRy ptouypos II y ER B OREus Gyllenhal: depressus, nigro-piceus, laevis, subnitidus, antennis pedibusque ferrugineis, femoribus interdum plus minusve infuscatis; thorace oblongo, crebre punctulato, medio obsolete canaliculato, lateribus rotundato, angulis posticis divaricatis acutis; elytris subovatis, thorace vix sesqui longioribus, apice acuminatis, profunde striatis, interstitiis subtiliter confertim punctulatis.

Longit. $3 \frac{1}{2}-3 \frac{3}{4}$ lin. Latit. $1 \frac{1}{3}-1_{\frac{5}{2}}$ lin.

Dejean, Cat. 3-ême édit. p. 105. - Germar, Zeitschrift. V. p. 137. 2. - Gebler, Bullet. de la Soc. de Moscou. 1847. II. p. 422. 4. - Gaubil, Cat. p. 110. 1. - Cat. Col. Europ. 1852. p. 48.

Elater hy penboreus Gyllenhal, Ins. Suec. IV.p. 350.13-14.Zetterstedt, Ins. Lappon. p. 145. 9. - Gebler in Ledebour Reise. II. 3. p. 83. 18.

IIypolituus plantus Eschscholt in Thon Entom. Archiv. II. 1. p. 34 .

Specimina in peninsula Kenai capta misit D. Ménétriés.

Occurrit etiam in Lapponia, Sibiria occidentali et orientali usque ad littora Maris Pacifici ut et in peninsula Kamschatka. 


\section{3}

192. Criptomypus ittoralis Eschscholtz: Mannerh. Bullet. de la Soc. de Moscou. 1846. II. p. 60. 5.

Var. b. tota fulvo-ferruginea, oculis solum nigris; specimen omnibus partibus induratum ileogue haud immaturum.

Ad ostia fl. Kaktnu peninsulae Kenai semel cum individnis genuinis capta. Invenitur haec species copiose sul) (runcis e mare rejectis in arena litorali jacentibus, exilu Junii. Etiam in insula liadjak mense Julio, sed minus frequenter, obvia; D. Holmberg.

193. CR Y P T OH Y PUS IMPIES I C OLL Is: convexus, nigrofuscus, opacus, supra subaeneus, fusco-pubescens; thorace latitudine longiore, lateribus subrectis, angulis posticis prominulis acutiuseulis, crebre punctulato, medio canaliculato, postice utrinque fovea oblonga obliqua impresso; elytris striatis, interstitiis subtiliter granulatis et dense punctulatis, epiplenris rufo - testaceis; pedibus ferrugineo - testaceis, femoribus infuscatis.

Longit. 3 lin. Latit. 1 lin.

Singulum, ad castellum Nicolajevsk in peninsula Kenai, specimen a D. Holmberg lectum.

A C. nocturvo Eschsch (Mannerh. Bullet. de la Soc. de Moscou. 1813. p. 239. 138.) differt statura convexiore, punctura multo crebriore, pubescentia fusca et thorace canaliculato, biimpresso, lateribus rectis haud rotundatis, angulisque posticis brevioribus, tenue tantum carinatis.

194. Crypto in y u s scarificat us Ménétriés: niger, sat crebre punctatus, longe el parce griseo-pubescens; thorace latitudine fere dimidio longiore, lateribus vix 


\section{4}

dilatato, antrorsum nomnihil angustato, angulis basalibus prominulis acutiusculis, postice in medio longitudinaliter impresso; elytris oblongis, striatis, ad suturam impressis; antennarum articulo primo, thoracis angulis posticis et margine inflexo, elytrorum limbo basali plus minusve late, margine laterali, epipleuris pedibusque rufo - ferrugineis.

Var. b. elytrorum limbo basali disco concolore, margine angustius rufescente; cetera ut in $a$.

Var. c. tota nigra, epipleuris pedibusque solum obscure rufescentibus.

Var. d. nigro-picea, antennis, thoracis angulis posticis, elytris totis pedibusque rufo-ferrugineis.

Longit. $2-2 \frac{1}{2}$ lin. Latit. $\frac{2}{3}-\frac{5}{4}$ lin.

Habitat in insula Kadjak et ad sinum Woskresensk peninsulae Kenai sub lapidibus locis aridis frequens; D. Holmberg. Individua quoque ex insula Kadjak, in Museo Acad. Imp. Scient. Petrop. asservata, communicavit D. Ménétriés. Var. $d$. ad castellum Nicolajevsk in Kenai a D. Holmberg rarius inventa, ubi etiam individua genuina sub lapidibus interdum occurrunt.

Cr. zimbato Mannerh. (Bullet. de la Soc. de Moscou. 1852. I. p. 327. 84.) affinis, sed longior, praesertim in elytris, paullo remotius punctulatus et thorax postice tantum longitudinaliter impressus.

195. Cryptohy Pn U FALAx: niger, parcius griseopubescens; thorace latitudine fere dimidio longiore, valde remote punctulato, lateribus subrectis, angulis basalibus prominulis acutiusculis, minime divaricatis, postice in medio longitudinaliter impresso; elytris oblongis, sublinearibus, striatis, ad suturam leviter 
impressis, interstitiis sat crebre punctulatis; antennarum articulo primo, thoracis angulis posticis et margine inflexo, elytrorum basi late, sutura, margine laterali et epipleuris pedibusque rufo-ferrugineis.

Longit. $2 \frac{1}{2}$ lin. Latit. $\frac{3}{4}$ lin.

Ad ostia fl. Kaktnu peninsulae Kenai, exitu Junii, sub lapidibus et lignis caesis rarius; D. Holmberg.

Praecedenti et Св. цимвато Mannerl. etiam valde propinquus, sed thorace angustiore, multo remotius punctato et elytris adhue longioribus, sublinearibus imprimis diversus.

196. Cryptohypus vestitus: subdepressus, nigro fuscus, longe et dense griseo-pubescens; thorace latitudine longiore, creberrime subtiliter punctulato, antrorsum vix angustato, lateribus parum rotundato, in medio distincte canaliculato, angulis posticis elongatis, acutis, divaricatis, apice incurvis; elytris leviter punctato-striatis, interstitiis subtiliter transversim strigosis, subrugulosis, macula humerali et margine laterali cum epipleuris rufo-testaceis, apice latius rufescenti-pellucidis; pedibus ferrugineo-testaceis.

Longit. $2 \frac{1}{2}$ lin. Latit. 1 lin.

Eodem loco uti praecedens species a 1). Holmberg rarissime lectus.

Forma thoracis angulisque ejus posticis Dracinтuum quodam modo mentiens, sed characteres generis Ciryptony pri.

197. Cryptouypus lucidude: convexiusculus, parce griseo-pubescens, supra obscure aeneus, violaceomicans, remote punctulatus, subtus nigro-piceus; thorace latitudine longiore, lateribus subrectis, an- 


\section{6}

gulis posticis acutis productis, haud divaricatis; elytris striatis, striis remote punctulatis; thoracis angulis posticis, antennis pedibusque ferrugineo-testaceis.

Longit. $2 \frac{1}{5}$ lin. Latit. $\frac{3}{4}$ lin.

Habitat ad sinum Woskresensk peninsulae Kenai, medio Junii, sub lapidibus frequens; D. F. Frankenhaeuser.

198. Cryptony pues REStrictulus: oblongiusculus, niger, tenue griseo-pubescens, subtiliter sat crebre punctulatus; thorace latitudine vix breviore, supra convexo, lateribus rotundato-ampliato et postice valde restricto, angulis acutiusculis productis; elytris in medio tantum obsoletissime substriatis; palpis, antennarum basi, sutura postice, margine et epipleuris elytrorum pedibusque rufo-ferrugineis, femoribus plus minusve infuscatis.

Longit. $1 \frac{3}{4}$ lin. Latit. $\frac{2}{5}$ lin.

Ad castellum Nicolajevsk peninsulae Kenai, medio Junii, sub lapidibus et lignis caesis a I). IIolmberg rarius inventus.

199. Corvanites lonatus Eschscholtz.

Diacanthus Lobatus Mannerh. Bullet. de la Soc. de Moscou 1846. II. p. 61.7.

Var.e. ut Var. d. sed elytrorum sutura nigrofusca.

Var. 7 . thoracis angulis posticis rufescentibus, elytris flavo - testaceis, pedibus ferrugineis, femoribus nonnihil infuscatis.

Specimen singulum Var. e. ad fl. Tschuniten peninsulae Kenai, initio Junii a 1). F. Frankenhaeuser inventum; 
Var. $/$ in insula Sitkha a D. Holmberg detecta. Individuat genuina et Var. $d$. etiam in insula Kadjak, mense Augusto, sub cortice pini rarius, et specimen hujus varietatis ad Neniłtschik in peninsula Kenai legit U. Holnherg.

200. Diacantius sericeus Gebler: depressus, nigropiceus, pube holosericea grisea variegatus, thorace subquadrato, latitudine paullo longiore, crebre punctulato, antice in medio subcarinato, lateribus leviter rotundato, angulis posticis parum productis, Iaud lisaricatis, acute: carinatis; elytris pone medium angusta-

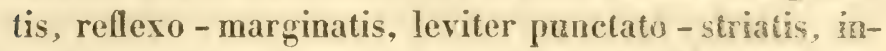
terstitiis subtiliter punctulatis; genubus, tibie tarsisgute ferrugineo-testaceis.

Longit. 5 lin. Latit. 2 lin.

Germar, Zeitschrift. IV. p. 69. 3.

Ludus Sericeus Eschscholt in Thon Entorr. Archis. It. I. p. 34. - Dejean, Cat. 3-ème édit. p. 106.

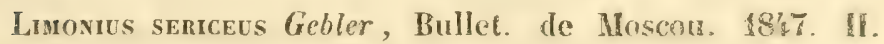
p. 416. 1.

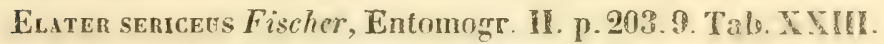
fig. 9. - Gebler, in Ledebour Reise II. 3. p. 8\%. 36.

IIabitat in peninsula Kenai, sec. D. Mémétriés, quui individuum ibi captum mihi benevole trarssissit. Adsunt in museo meo specimina e Sibiria occidentali et peninsula Kamschatka.

201. Diacantuus ingGoratus: convexus, inflatus, migro-piceus, subnilidus, griseo-pubescens, thorace elytris haud angustiore, longritudine parum latiore, antrorsum rotundato, crebre punctato, angulis posticis triangularibus acutis, vix divaricatis, impresibuse obliqua leviore utrinque sublimitatis; scutello arute 
carinato; elytris crebre confuse punctatis, basi breviter striatis, dein obsolete costatis, macula rotundata basali, margine laterali cum epipleuris, ad apicem in maculam majorem subhamatam dilatato, abdominis segmentorum marginibus, tibiis tarsisque rufo-ferrugineis.

Longit. $4 \frac{1}{3}$ lin. Latit. $1 \frac{3}{4}$ lin.

Ilabitat in insula Kadjak rarissime; D. Holmberg.

Magnitudo et statura D. costalis Payk. omnino; colore autem minus aeneo, thoracis punctis nonnihil majoribus angulisque posticis paullo latioribus, minus divaricatis, impressione vix conspicua a disco limitatis (in costali thorax medio depressus, obsolete subcarinatus), scutello per totam longitudinem carinato elytrisque aliter coloratis diversus.

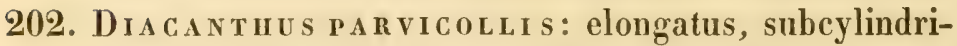
cus , convexus, fusco-aeneus, longe griseo-pubescens; thorace elytris quadruplo breviore et illis angustiore, ex angulis posticis antrorsum modice rotundato - angustato, crebre punctulato, angulis posticis triangularibus, divaricatis, subauriculatis, impressione utrinque profunda obliqua a disco limitatis; scutello acute carinato; elytris crebre confuse punctulatis, basi breviter striatis, dein obsoletissime costatis, margine laterali cum epipleuris rufo-ferrugineo; genubus, tibiis tarsisque ferrugineo - piceis.

Var. b. elytrorum macula in utroque basali, sutura angustissime, margine laterali cum epipleuris, ut et segmentorum ventralium marginibus fulvis; $t i-$ biis extrorsum tarsisque obscurius ferrugineis.

Longit. $3 \frac{1}{2}-3 \frac{2}{3}$ lin. Latit. $1 \frac{1}{3}-1 \frac{2}{3} \operatorname{lin}$. 
Habitat in peninsula Kenai, rarius, ubi ad f1. Tschunuktnu, medio Junii, legit D. F. Frankenhaeuser et Var. $b$. ad ostia fl. Kaktnu cepit D. Holmberg.

Specimina duo a me visa masculini sexus maribus D. costalis Payk. certe similia, sed thorax paullo profundius et remotius punctatus, angustior, antrorsum magis attenuatus, angulis posticis evidentius divaricatis, extrorsum prominulis et scutellum per totam longitudinem carinatum. Anne mas D. Decorati a me jam heic descripti? D. singulari Manncrh. (Bullet. de la Soc. de Moscou. 1852. II. p. 290. 34.) e portu $\Lambda$ jan etiam statura affinis, sed multo major, magis elongatus et variis notis ab illo distinguendus.

203. Diacantius confluens Gebler: nigro-aeneus, opacus; thorace subquadrato, latitudine vix longiore, creberrime confertim punctato, per totam longitudinem in medio carinato, lateribus antrorsum modice rotundato-angustato, angulis posticis divaricatis productis, acute carinatis; elytris laete viridi-aeneis, profunde seriatim lacunosis, impressionibus plus minusve linearibus, interstitis irregulariter punctulatis, praesertim postice vario modo transversim plicatis.

Longit. $6 \frac{1}{2}$ lin. Latit. $2 \frac{1}{3}$ lin.

Elater confuens Gebler in Ledebour Reise. II. 3. p. 80. 9.

Exemplare in peninsula Kenai captum e Museo Acad. Imp. Scient. petrop. communicavit I). Ménétriés. Occurrit in Sibiria e montibus Altaicis usque ad littora Maris Pacifici.

A D. nugoso Bonelli thorace paullo longiore, angulis posticis magis elongatis elytrisque profundius et magis irregulariter lacunosis, interstitiis transversim plicatis diversus. 


\section{Y P H O N I A.}

204. CyPHON VARIBilis Thunberg: ovalis, nigro-fuscus, breviter griseo-pubescens; antennis fuscis basi pallidis; thorace brevissimo, basi bisinuato, livido - testaceo, subtilissime punctato; elytris livido-testaceis, crebre punctatis; tibiis testaceis.

Longit. $1 \frac{1}{2}$ lin. Latit. 1 lin.

Redienbacher, Fauna Austr. p. 315.

Filodes varuabis Guérin-Méneville, Species el Jconogr. des Anim. art. G. Elodes. p. 4. 5. - Gaubil, Cat. p. 1133.

Cintharis vakiabis Var. Thunberg, Mus. Ups. 4. 54. (1787.) Cistela punescens Fabricius, Ent. Syst. II. p. 4.5.

Crphon pubescexs Fabricius, Syst. El. I. p. 502. 4. - Gyllenhal, Ins. Snec. I. 369. 4. - Sahlberg, Ins. Fenn. I. p. 117. L. - Stephens, Ill. Mandib. III. p. 285. - Zetterstedt, Fauna Lappon. I p. 120. 2. Ins. Lappon. p. 87. 3. Gebler, Bullet. de la Soc. de Moscon. 1847. II. p. 428. 3. Cat. Col. Europ. 1852. p. 49.

Elodes pubescens Bach, Käferfauna. III. p. 5̆ł. 3.

Crmox donsals Schönherr, Syn. Ins. II. p. 322. - Stephens, Ill. Mandib. III p. 286.

Crpmon ovmes Say Journ. of Acad. Nat. Scienc. of Philadelphia. V. p. 161.

Crpuns roscicers Kirby, in Richardson Fauma Bor. Amer. IV. p. 24.5. 335.

Cistela meta Olivier, Entom. III. 54. p. 9. Tab. 1. fig. 12.

Creprocephumes donsals Marsham, Entom. Brit. I. 210.

Ad rivulum II. Tschunuktnu peninsulae Kenai, initio Julii, in volatu a D. E. Frankenhaeuser semel captus. 


\section{1}

\section{T E L E P H O R A}

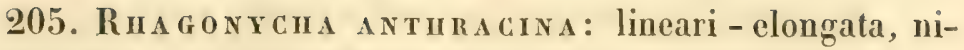
gra, opaca, creberrime rugoso-punctata; ore, antennarum articulo primo subtus geniculisque obsolete testaceis; thorace elytris multo angustiore, subquadrato, disco aequali, basi tantum marginato, angulis posticis subrectis; elytris lineis binis elevatis, vix conspicuis.

Longit. 3 lin. Latit. $\frac{5}{6}$ lin.

In gramine et in ligno putrido, ad fl. Skeljanktnu peninsulae Kenai, medio Junii, non infrequens; D. E. Frankenhaeuser.

\section{P T I N I A.}

206. Dinoderus sus tritus Paykull : lineari - elongatus, cylindricus, nigro-piceus, opacus, pubescens; antennarum apice pedibusque rufo - piceis; thorace suborbiculato, tuberculis minutis crebre granulato, antice et lateribus denticulis numerosis armato, elytris punctis majoribus profunde impressis dense subseriatis, rugis transversis et ad basin tuberculis valde scabratis.

Longit. $1 \frac{2}{3}-2 \frac{1}{4}$ lin. Latit. $\frac{1}{2}-\frac{3}{4}$ lin.

Stephens, III. Mandib. III. 352. Manual. p. 20\%. 1622. Gaubil, Cat. p. 12כ. 1. - Cat. Col. Europ. 1852. p. 5้4.

Apate substrita Paykull, Fauna Suec. III. p. 142. 2. Gyllenhal, Ins. Suec. III. p. 37\%. 2. - Gebler, in Ledebour Reise. II. 3. p. 179. 3. - Zetterstedt, Fauna Lappon. I. p. 349. 1. Ins. Lappon. p. 194. 1. - Sahlberg, Ins. Femn. II. p. 155. 1. - Dejean, Cat. 3-ème édit. 1. 33\%. - Germar, Fauna Ins. Eur. XX. 12. - 
Redtenbacher, Fauna Austr. p. 35̌. - Bach, Käferfauna, III. p. 117.3.

Ad sinum Woskresensk peninsulae Kenai, exitu Maji, in pinis recenter decorticatis copiose legit D. F. Frankenhaeuser.

207. Cis EPH P I A TUS: breviusculus, convexus, nigro piceus, brevissime griseo-pubescens ; capite rufo, fronte transversim impressa, margine antico bidentato, vertice foveola impressa; thorace inermi, subtilissime dense punctulato, antrorsum rotundato-angustato, apice truncato; elytris thorace sesqui longioribus, profunde et remote subseriatim punctatis, rufis, in medio macula magna communi transversa, marginem utrinque attingente, nigra; pedibus rufis.

Longit. 1 lin. Latit. $\frac{1}{2}$ lin.

In insula Sitkha a D. Holmberg semel captus.

\section{B OS T R I C H I N A.}

208. Bostricuus inter rupus Mannerheim: Bullet. de la Soc. de Moscou. 1852. I. p. 357. 147.

Var. c. elytris pedibusque rufo-ferrugineis; cetera ut in $a$ et $b$.

Var. d. tota rufo-ferrugined.

Etiam in insula Sitkha rarius obviae. Individua genuina ad castellum Nicolajevsk peninsulae Kenai sat frequenter quoque occurrunt, exitu Jumii, in truncis caesis pini; D Holmberg.

209. Bostricius concineus Mannerheim: Bullet. de la Soc. de Moscou. 1852. I. p. 358. 149. 


\section{3}

$V a r . b$. tota rufo-castanea.

Var. $c$. pallide testacea, elytris plus minusve apice infuscatis.

Var. d. obscure rufo-castanea, thorace jam magis jam minus infuscato.

Varietates $b$ et $c$ in insula Kadjak sub cortice pini sat copiose inventae. Var. $d$. ad Castellum Constantinovsk in insula Chtagaluk una cum individuis genuinis et Var. $c$. initio Junii capta, ubi haec species in lignis caesis etiam frequenter occurrit; D. Holmberg.

210. Bostricius SePtentrionis Leconte: Mannerh. Bullet. de la Soc. de Moscou. 1843. p. 298. 261.

Var. b. nigro-picea, antennis pedibusque rufopiceis.

Var. c. ferrugineo-testacea.

Var. d. pallide luteo-testacea.

In insula Sitkha cum a D. Holmberg inventac. Occurrit haec species etiam circa castellum Constantinovsk ad si num Nutschek in insula Chtagaluk, in peninsula Kenai et in insula Afognak.

211. BostricuUs AFF B R Mannerheim: Bullet. de la Soc. de Moscou. 1852. I. p. 359. 151.

Var. b. rufo-castanea.

Ad sinum Woskresensk peninsulae Kienai, ubi etiam individua genuina, medio Maji, sub cortice pini sat frequenter obvia, a D. Holmberg lecta.

212. CR Y PI A L U S S TR I T UL U s: oblongus, fuscus, opacus, pube cinerea dense vestitus; thorace pulvinato, tuber- 


\section{4}

culis exasperato, antrorsum densioribus; elytris evidenter punctato-striatis, interstitiis subtiliter rugulosopunctatis; antennis pedibusque piceis.

Var. b. fusco-castanea; thorace rufescente, glabriusculo; elytris opacis, subsericeis; antemnis pedibusque rufo-piceis.

\section{Longit. $\frac{5}{6}$ lin. Latit. $\frac{1}{2}$ lin.}

Ad lacum Skeljamna peninsulae Kenai, medio Junii in volatu a D. F. Frankenhaeuser semel inventus. Var. b. ad castellum Nicolajevsk ejusdem peninsulae, exitu Junii, sub ligno a D. Holmberg rarissime capta.

Cr. Granulato Ratzeburg longior, thoracis tuberculis majoribus, densioribus et elytris evidenter punctatostriatis diversus.

213. X y loterus bivit a tus Kirby: cylindricus, nigropiceus; thorace subgloboso, opaco, antrorsum tuberculato - scabro, basi anguste rufo-ferrugineo; elytris leviter striato - punctatis, vittis duabus longitudinalibus, apice conniventibus; antennis pedibusque luteis.

Var. b. elytris pallide luteis, lineola marginali ante apicem evanescente nigra; cetera ut in $a$.

Longit. $1 \frac{5}{4}$ lin. Latit. $\frac{2}{3}$ lin.

Apate mivittata, Richardson Fauna Bor. Amer. IV. p. 192. 256. Tab. VIII fig. 5.

Ilabitat ad sinum Woskresensk et ad ostia fl. Kaktnu peninsulae Kenai, mensibus Majo et Junio, sub cortice et in ligno pini non infrequens; D. Holmberg.

A Bostricho cavifnonte Mannerh. (Bullet. de la Soc. de Moscou. 1843. p. 297. 260. 1852. I. p. 3399. 153.), cui affinis, differt statura nonnihil longiore, thorace antror- 


\section{5}

sum evidentius tuberculato, elytris distinctius striatopunetatis, punctis striarum minoribus et eorum vittis nigris apicem usque protensis.

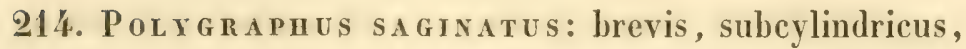
fusco-castaneus; thorace longitudine latiore, antrorsum latitudine baseos fere duplo angustiore, creberrime punctulato, medio carinato, carina laevigata, apice oblique truncato; elytris seriatim subtiliter transverse rugulosis, pilis cinereis brevissimis praesertim postice obsitis, margine basali anguste et acute elevato; pedibus rufis.

Longit. $1 \frac{1}{3}$ lin. Latit. $\frac{2}{5}$ lin.

In regionibus interioribus peninsulae Kenai a D. F. Frankenhaeuser semel inventus.

215. II y L E IN U R R F IPENN IS Kirby: breviusculus, niger, confertissime ruguloso-punctatus, pilis depressis in thorace et elytris squamaeformibus cinereis obductus; capite inter oculos obsolete bituberculato; thorace antrorsum angustato, fere constricto, dorso longitudinaliter carinato; elytris rufo - castaneis, seriebus aliquot e punctis magis regulariter dispositis substriatis, basi tuberculis minutis exasperatis; antennis pedibusque rufescentibus.

Longit. $1 \frac{1}{4}$ lin. Latit. $\frac{1}{2}$ lin.

Apate (Lepisonus) rufipensis hioby in Richardson Fauna

Bor. Amer.: IV. p. 193. 258. Tab. VIII. fig. 2.

Ad rivulum fl. Tschunuktnu peninsulae Kenai, mense Augusto, sub cortice arboreo specimen singulum legit D. F. Frankenhaeuser. 


\section{6}

216. Hycurgus obesus Eschscholtz: Mannerh. Bullet. de la Soc. de Moscou. 1843. p. 296. 257. 1852. I. p. 356.145 .

Var. d. rufo-castanea, capite corporeque subtus infuscatis.

Specimen hujus varietatis cum individuis genuinis in insula Atkha captum communicavit D. Ménétriés.

217. Hylurgus rufipennis Kirby: oblongus, ater, confertim ruguloso - punctatus, longe fusco - pilosus; thorace latitudine vix breviore, antrorsum angustato, ibique transversim impresso, constricto, apice emarginato, margine rufo, basi bisinuato, dorso longitudinaliter tenue carinato; elytris rufis, profunde sulcatocrenatis, interstitiis latis, dense tuberculato-scabris; antennis pedibusque piceis, tarsis rufis.

Var. b. tota nigra.

Longit. $2 \frac{3}{4}-3$ lin. Latit. $1 \frac{1}{4}-1 \frac{1}{3}$ lin.

Richarlson Fauna Bor. Amer. IV. p. 195. 261.

Inabitat ad sinum Woskresensk et ad castellum Nicolajevsk peninsulae Kenai, mense Junio, in ligno nuper caeso frequens; ad castellum Constantinovsk in insula Chtagaluk autem rarius; DD. Holmberg et F. Frankenhaeuser.

Praecedenti valde similis, sed thorace multo crebrius punctato, elytrorumgue striis profundius exaratis et interstitiis densius tuberculato-scabris diversus.

218. Hylastes rugipenis Mannerheim.

Hyourgus rugirennis: Mannerh. Bullet. de la Soc. de Moscou. 1843. p. 297. 258. 


\section{Var. $b$. tota pallide ferrugineo-testacea.}

In insula Kadjak, ubi haec species sub cortice pini vulgaris, a D. Holmberg inventa.

219. II YLASTES S B Costulatus: oblongus, subcylindricus, nigro-piceus, squamulis griseis erectis parce adspersus, thorace antrorsum angustato, haud constricto, subtiliter crebre punctulato, medio carinato ibique laevigato; elytris rugulosis, tuberculis seriatis subcostatis; antennis pedibusque ferrugineis.

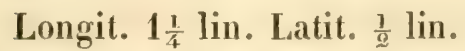

Ad ostia fl. EKaktnu peninsulae Kenai a I). Holmbery rarissime captus.

220. HYLASTES CRISt A US: oblongus, niger, parce cinereo - setulosus, thorace longitudine breviore, profunde punctato, subrugoso, antrorsum angustato, lateribus rotundato, basi bisinuato; elytris tuberculatorugosis, subcostatis, basi crista transversa valde elevata e spinulis acutis congregatis.

Longit. 1 lin. Latit. $\frac{5}{1 \frac{9}{2}}$ lin.

In regionibus interioribus peninsulae Kenai a D. F. Frankenhaeuser semel captus.

\section{U R G U L I O N I A.}

221. RHY colus BRUnNeus Eschschollz: Mannerh. Bull. de la Soc. de Moscou. 18ł3. p. 295. 255.

Var. b. pallide rufo-testacea.

Ad sinum Woskresensk peninsulac Kenai, exitu Maji, sub ligno specimen singulum legit D. $F$. Frankenhaeuser. 


\section{8}

222. Tra cirodes Ptinoides Eschscholtz: Mannerh. Bull. de la Soc. de Moscou. 1843. p. 292. 249.

Var. b. ferrugineo - castanea, antennis pedibusque dilutioribus.

In insulis Kadjak et $\Lambda$ fognak, ut et ad ostia $\mathbb{1 l}$. Kaktnu peninsulae Kenai a D. Holmberg lecta.

IIabitat haec species per totam aestatem frequens etiam in insula Kadjak ubique et in locis litoralibus peninsulae Kenai, sub lignis e mare rejectis degens.

223. Eririnus a r io : oblongus, niger, glaber, nitidus; antennarum scapo rufo, funiculo castaneo-pubescente; rostro longissimo, arcuato; thorace latitudine vix longiore, lateribus rotundato, antice angustato, subconstricto, profunde punctato, linea media antrorsum laevigata subcarinato, margine antico rufescentipellucido; elytris oblongo-ovalibus, profunde punctatostriatis, interstitiis punctulatis; femoribus muticis; tibiarum apice tarsisque rufis.

Var. b. tota rufo-ferruginea; certe immatura.

Longit. sine rostro $2 \frac{1}{2}-3$ lin. Latit. $1-1 \frac{1}{4} \operatorname{lin}$.

Jabitat ad ostia fl. Kaktnu peninsulae Kenai, exitu Junii, sub lignis e mare rejectis vulgaris, in insula Kadjak vero rarius, D. Holmberg.

E. aетuopi Fabr. primo intuitu simillimus, at accuratius examinatus revera ab illo distinctus; thorax nempe aliter constructus, brevior, latior, lateribus dilatatus, antice subconstrictus et elytra longiora.

224. Eriruinus rufulus: oblongus, rufo - testaceus, subnitidus, parce griseo-pubescens; oculis nigris; rostro longissimo, parum arcuato; thorace elytris multo 
angustiore, latitudine vix breviore, lateribus rotundavo, crebre profunde punctato; elytris oblongis, profunde punctato-striatis, interstitiis punctulatis, fasciis transversis angustis e pilis griseis condensatis hinc inde obsolete ornatis; femoribus muticis.

Longit. sine rostro 2 lin. Latit。 $\frac{\pi}{5}$ lin.

Ad sinum Woskresensk peninsulae Kenai, medio Junii , in salicetis a D. F. Frankenhaeuser sat frequenter captus.

225. Erirhin U S LURy d S ; oblongus , rufo-castaneus, tenue griseo-pubescens; rostro longo arcuato cum capite nigro-fusco; thorace elytris multo angustiore, latitudine breviore, lateribus rotundato, ad angulos posticos utrinque impresso, nigro - fusco, antice posticeque rufo - castaneo; elytris oblongo - ovatis, profunde punctato - striatis, interstitiis rugulosis, rufo - castaneis, ante apicem utrinque tuberculo instructis, maculis nigro - fuscis et cinereo - pallidis e pilis condensatis variegatis; femoribus dentatis.

Longit. sine rostro $1 \frac{1}{\frac{1}{3}}$ lin. Latit. $\frac{2}{3}$ lin.

lisdem locis uti praecedens species eodemque tempore a D. F. Frankenhacuser sat copiose lectus.

E. raennato Payk. affinis, sed multo brevior et profundius punctatus.

226. Eriruinus subignatus: elongatus, rufo-castaneus, dense griseo - pubescens; rostro valido longissimo vix arcuato; capite nigro; thorace elytris angustiore, latitudine nomnihil breviore, lateribus modice rotundato, crebre punctulato, transversim infuscato; 


\section{0}

elytris oblongis, profunde punctato - striatis, utroque maculis tribus longitudinaliter dispositis nigro-fuscis; femoribus dentatis.

Var. $b$. denudata, thorace immaculato, elytrorum maculis nigro - fuscis evidentioribus.

Longit. sine rostro $1 \frac{1}{4}$ lin. Latit. $\frac{1}{2}$ lin.

Etiam in salicetis ad sinum Woskresensk peninsulae Kenai, medio Junii, frequenter obvius; D. F. Frankenhaeuser.

Statura et magnitudo E. sasicisi Gyllenh. sed thorace minore, elytris majoribus, longioribus, pubescentia dersiore et maculis tribus fuscis elytrorum ab illo dignoscendus.

227. Eriruinus vestitus: oblongo - ovatus, nigropiceus, griseo - pubescens; rostro breviore, valido, parum arcuato; thorace latitudine breviore, lateribus modice rotundato, profunde punctato, lineis quatuor longitudinalibus e pube grisea condensata; elytris latis, subovatis, profunde punctato - striatis, interstitiis subtiliter punctulatis, dense griseo-pubescentibus, maculis paucis nigris irregularibus denudatis; femoribus obtuse dentatis.

Var. b. rufo-ferruginea vel pallide testacea, capite infuscato.

Longit. sine rostro $1 \frac{1}{6}$ lin. Latit. $\frac{5}{1}$ lin.

Simul cum speciebus tribus proxime praecedentibus non infrequens, Var. b. vero rarissime occurrit; D. F. Frankentaeuser.

E. majali $\boldsymbol{P}_{a y j k}$ in elytris multo latior, pubescentia grisea magis condensati, praesertim in elytris, relictis tantum maculis paucis obsoletis denudatis. 


\section{1}

228. Pritoxomus seriatus : oblongus, niger, dense brunneo - aureo - pubescens et squamulosus; antennis pedibusque rufescentibus; rostro breviore, parum arcuato; thorace crebre punctulato, convexo, utrinque antrorsum rotundato, lineis tribus e pilis pallidioribus lavescentibus potato; elytris subtiliter tenue striato-punctulatis, sutura infuscata, interstitiis alternis 3,5 et 7 nonuihil elevatioribus pilis flavo-griseis densius obductis, $1,3,5$ et 7 maculis nigris seriatis.

Longit. sine rostro 3 lin. Latit. $1 \frac{1}{3}$ lin.

Ilabitat in insula Kadjak, ubi mense Augusto exemplare singulum in baccis Rubi cepit D. Holmberg.

Pи. suspicioso Herbst, Schönh. (Miцт1 Gyllenh.) affinis, sed thorace angustiore, densius squamoso, subtilius punctato, ante medium et non in ipso medio rotundato-dilatato, elytrisque longioribus, levius striato - punctatis, maculis nigris in series quatuor regulares dispositis ab illo cignoscendus.

229. LEPYR S GEMELus Kirby : Mannerh. Bullet. de la Soc. de Moscou. 1852. I. p. 351. 136.

Hylobius Lineatus Motschulsky in litteris.

Individuum ad fl. Kwichpakh captum, nomine citato imposito, e museo suo amice communicavit D. Motschulsky.

230. LePID OPIORUS LINEATIColis Kirby: oblongo ovatus, niger, corpore subtus capiteque cum rostro dense griseo-squamulosis, thorace latitudine longiore, lateribus modice rotundato, crebre confertim ruguloso, in medio longitudinaliter obsolete canaliculato, vitlis tribus angustis, una mediana, ceteris lateralibus e squamulis albidis condensatis; elytris striis profunde 
exaratis et in striis remote punctatis, interstitis crebre rugulosis, squamulis griseis et brunneis variegatis, versus apicem seriation setulosis; antennis pedibusque plus mimusve rufescentibus, femoribus mulicis, saepe obscurioribus.

Longit. cum rostro $2 \frac{1}{4}-2 \frac{1}{2}$ lin. Latit. $\frac{5}{6}-1 \mathrm{lin}$.

Richardson, Fauna Bor. Amer. IV. p. 201. 269. - Schünherr, Gen. et Spec. Curcul. VI. p. 256. 1. - Jeliel, Cat. Curcul. p. 105.

Strongulopithamus bonealis Ménétriés in litteris.

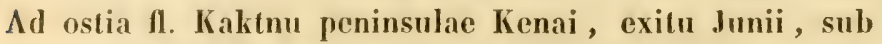
truncis pini recenter caesis decorticatis, in gramine jacentibus, copiose invenit D. Ilolmberg. Specimen ad A. Kwichpakh captum etiam communicavit D. Ménétriés.

231. Liofilone inguinatus Mannerheim: Bullet. de la Soc. de Hoscou. 1852. 1. p. 351. 135.

Ströngy lopithalmus tesselcatus Motschulsky in lilteris.

Eliam in insulis Kadjak et Afognak a D. Holmberg lectus. Individua ex insulis Atkha el Unga allata, in Museo Acad. Imp. Scient. Petrop. asservata, transmisit D. Ménćtriés.

232. Aropius a ternates Say: Mannerh. Bullet. de la Soc. de Moscou. 1843. p. 290. 244.

Schünherr, Gen. et Spec. Curcul. VI. p. 204. 2. - Jekel, Cat. Curcul. p. 101.

IIrpsonotus altennitus $S_{a y}$, Deser. of Curcul, of North America p. 10.

Var. b. Mannerh. 1. c.

Lopmoevs mnнoвates Motschulsky in litteris. 
Var.c. maculis elytrorum nigris cum pallidis e squamulis viridi-cyanescentibus alternatis, maculis duabus majoribus in utroque obsoletioribus e squamulis ejusdem coloris.

In insula Kadjak a D. IIolmbery semel inventa.

Specimen Var. b. maculis elytrorum minoribus ibidem captum etiam benevole communicavit D. Motschulsky.

233. A L O P U S S R I T US: subovalus, niger, fusco-cupreosquamosus; rostro profunde canaliculato; thorace latitudine breviore, crebre ruguloso, ante medium lateribus rotundato - dilatato, dorso carinula clevata, antice posticeque abbreviata, instructo; elytris subliliter punctato-striatis, maculis nigro-fuscis supra strias seriatis et his maculis cum aliis e pilis albescentibus obsoletis altermatis, interstitiis confertiu granulatis.

Longit. cum rostro $5 \frac{1}{4}-5 \frac{1}{2}$ lin. Latit. $2 \frac{1}{6}-2 \frac{1}{4}$ lin.

Iabitat in insula Kadjak rarissime, in peninsula Kenai ad castellum Nicolajevsk, exitu Junii, non infrequens el ad sinum Woskresensk rarius, sub truncis putrescentibus victitans; D. Holmberg.

Praecedente brevior, rostro et thorace brevioribus, hoc acutius carinato, antrorsum lateribus dilatato et maculis majoribus niveo-argenteis elytrorum deficientibus ab illo diversus.

\section{R H I N O S I M I A.}

234. RIIN OS I US A E N EIR OST R Is : supra aenco-cupreus, nitidus, subtus piceus; rostro elongato, plano, apice parum dilatato, cum capite crebre et profunde pun- 
ctato, ore rufo-ferrugineo; thorace convexo, lateribus parum rotundato, profunde punctato, in disco foveolis quatuor fere in quadrangulum dispositis obsolete impressis; elytris oblongis, pone basin transversim impressis, striato-punctatis, striis basi profundius, posterius tenuius punctatis; pedibus rufo-testaceis.

Longit. cum rostro $1 \frac{1}{2}$ lin. Latit. $1^{\frac{5}{2}}$ lin.

Ilabitat in insula Sitkha; D. Ferd. Sahlberg. Ad rivulos fl.

Tschunuktnu peninsulae Kenai, medio Julii, in volatu a D. F. Frankenhaeuser captus. Rarissime occurrit.

Rir. pLannostm Fabr. similis, sed ob rostrum longius, angustius, aeneo-cupreum, thoracem angustiorem, et elytrorum strias posterius tenuiores ab illo removendus.

\section{E R A II B Y C I N A.}

235. Callidium cicatricosum: depressum, supra obscure aeneo-subvirescens, opacum, parce nigro-pilosum, subtus nigro - aeneum, magis nitidum, antennis pedibusque nigris; thorace elytris angustiore, brevi, transverso, crebre rugoso, basi apiceque truncato, lateribus valde rotundato; elytris linearibus, apice singulatim rotundatis, supra reticulatim rugosis vel varioloso-cicatricosis.

\section{Longit. 5 lin. Latit. $1 \frac{5}{6}$ lin.}

Ad ostia fl. Kaktnu peninsulae Kenai, exitu Junii, in ligno nuper caeso a D. Holmberg rarius lectum.

C. dicatato Payk. propinquum, sed magis lineare, obscurius aeneo - virescens, opacum, thorace levius rugoso, elytris reticulo densiore minus inaequaliter rugosis vel per totam superficiem varioloso-cicatricosis (in DiLatato elytra basi punctata et ante medium posterius inaequaliter valde elevato-nervosa), apice magis rotundatis, antennis pedibusque nigris discrepans. 
236. Callidium proteus Kirby: nigrum, punctulatum, griseo-pilosum; thorace longitudine sesqui latiore, laete violaceo, lateribus valde rotundato ibique utrinque punctis confluentibus scabro; elytris posterius nonnihil angustatis, rugoso-punctatis, apice rotundatis utroque margine lineisque duabus callosis, versus apicem plus minusve abbrevialis, eburneo-albidis; femoribus rufo-ferrugineis.

Longit. 6 lin. Latit. 2 lin.

Callidum (Menum) proteus Kirby in Richardson, Fauna Bor. Amer. IV. p. 172. 228. Tab. V. fig. 5. - Haldemen, Materials towards a IIistory of the Coleoptera Longicornia of the United States (Trans, of the Americ. Philo. sophical Society. Vol. X.) p. 37. 50.

Individuum ad fl. Kiwichpakh captum ad describendun misit D. Ménétriés.

237. Criomorpus cinanopterus Kirby: linearis, niger, griseo-pilosus; capite longitudinaliter canalicuto, remote punctato; thorace longitudine vix latiore, basi apiceque truncato, lateribus in medio nonnihil dilatatorotundato, disco longitudinaliter late excavato ibique remote, versus latera autem confertim punctato; elytris linearibus, castaneis, subtiliter griseo-pubescentibus, apice subtruncatis; pedibus rufis, femoribus valde incrassatis, haud clavatis.

Longit. $4 \frac{1}{2}$ lin. Latit. $1 \frac{1}{3}$ lin.

Callodum (Теtropum) cinsumopterum Kirby in Richardson, Fauna Bor. Amer. IV. p. 174. 231. Tab V. fig. 8. Laldeman, Col. Longicorn. of the Unit Stat. p. 37. 36. 


\section{6}

Ilabitat ad 11. Kwichpakh, sec. D. Ménétriés, qui specimen e Museo Acad. Imp. Scient. Petrop. amice communicavit.

A descriptione Kirbyana differt tantum antennis totis nigris, ideoque ut hujus species varietas considerandum est exemplare a me examinatum, quum in ceteris omni modo quadrat.

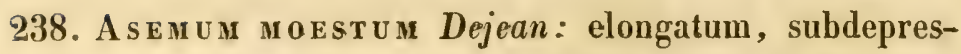
sum, atrum, opacum, breviter nigro-pubescens; thorace suborbiculari, apice leviter emarginato, basi truncato, supra crebre granulato, in medio obsolete longitudinaliter excavato; ely tris subtilissime confertim granulatis et strigulosis, hinc inde transversim rugosis, costis quatuor elevatis, a sutura dimidiam partem disci occupantibus, quarum alternis altioribus, secundaque cum quarta ante apicem connivente.

Longit. $5 \frac{1}{2}$ lin. Latit. $1 \frac{3}{4}$ lin.

Dejean, Cat. 3-ême édit. p. 35\%. - Haldenan, Col. Longicorn. of the Unit. Stat. p. 35. 39.

Calcidum striatom Kirby in Richardson, Fauna Bor. Amer. IV.p. 171: 226.

Ad rivulum fl. Tschunuktnu peninsulae Kenai, initio Julii, in cortice populi semel invenit D. F. Frankenhaeuser.

A. striato Linné valde affine, sed differt statura fere depressa, thorace fortius granulato et elytris tantum prope suturam ad dimidiam partem disci costatis.

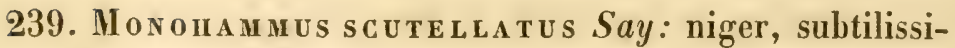
me griseo-pubescens, capite thoraceque remote profunde punctatis, hoc lateribus utrinque spina valida apice obtusata armato; scutello niveo-tomentoso; ely- 
tris confluenter punctatis et rugosis; eorum summo margine, antennis pedibusque rufescentibus. (Mas.)

Femina differt statura magis cylindrica, elytris maculis griseis hine inde conspersis et antennis brevioribus, articulis cinereo-annulatis.

Longit. $9-9 \frac{3}{4}$ lin. Latit. $2 \frac{3}{4}-3$ lin.

IIaldeman, Col. Longicorn. of the Unit. Stat. p. 51.156.

Monohumus faber Dejean, Cat. 3-ême édit. p. 366.

Monochamus nesutor Kirby in Richardson, Fauna Bor. Amer. IV. p. 167. 221.

Cerambyx scutellatus Say in Long's Expedition II. p. 289.

Habitat ad fl. Kwichpakh, sec. D. Ménétriés; Mus. Acad. Imp. Scient. Petrop.

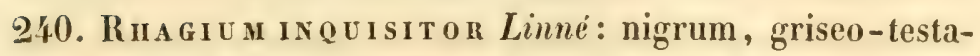
ceo-pubescens; capite quadrato, profunde canaliculato, in medio dense pubescente, remote nigro - punctato, pone oculos denudato; thorace basi apiceque constricto ibique transversim carinato, lateribus utrinque spina acuta armato; elytris rugoso-punctatis, maculatim pubescentibus, posterius parum angustatis, apice rotundatis, dorso utrinque leviter tricostatis, costa externa ante medium evanescente, fasciis duabus transversis oblique parallelis, fere in medio approximatis, testaceis, interjecta macula laterali nigra.

Longit. 6 $\frac{1}{2}$ lin. Latit. $2 \frac{1}{4}$ lin.

Fabricius, Syst Ent. p. 182. 1. Ent. Syst. I. 2. p. 304. 2. Syst. EI. II. p. 313. 2. - Paykull, Fauna Suec. III. 


\section{8}

p. 66. 1. - Panzer, Fauna. 82 4. - Schönherr, Syn. Ins. III. p. 412. 3. - Gyllenhal, Ins. Suec. IV. p. 45. 2.Stephens, Ill. Mandib. IV. p. 254. Manual p. 277. 2166. Zetterstedt, Fauna Lappon. I. p. 372. 1. Ins. Lappon. p. 206. 1. - Dejean, Cat. 3-ème édit. p 380. - Mulsant, Hist. nat. des Col. de France. Longicorn. p. 225. 3. Gebler, Bullet. de la Soc. de Moscou. 1848. I. p. 410. 3Redtenbacher Fauna Ausir. p. 503. - Gaubil, Cat. p. 182. 4. - Catal. Col. Europ. p. 86.

Cerambx inquisitor Var. $\beta$. Linné Fauna Suec. 659. Syst. Nat II. p. 630. 49.

Stenochonus mordax Olivier, Entom. IV. 69. p. 7. 1. Tab. 2. fig. 12.

Specimen in insula Sitkha a se captum mihi transmisit D Ferd. Sahlberg. Illud in statu larvae vel pupae cum lignis ex Europa navibus verisimiliter importatum.

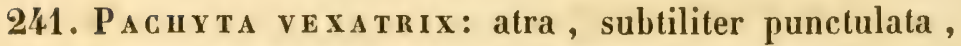
dense griseo-pubescens; thorace latitudine dimidio longiore, campaniformi, apice truncato, lateribus in medio nonnihil dilatato, basi leviter bisinuato, angulis extrorsum prominulis, acutiusculis, supra omnino aequali; elytris postice valde angustatis, dehiscentibus, apice ipso subtruncato, flavo-albidis, crebre punctatis, sutura, margine laterali, basi anguste, maculis tribus oblongis fere transversim positis ante medium, quarum externis contiguis, duabus fere in medio, quarum interiore majore obliqua apiceque late nigris, nigredine hac antice unidentata.

$V a r$. b. limbo basali nigro cum maculis externa et interna fasciae macularis anterioris et macula hac cuu sutura connexis.

Longit. $4-6$ lin. Latit. $1 \frac{1}{5}-2$ lin. 
Leptura sexmaculata? Kirby in Richardson, Fauna Bor. Amer. IV. p. 182. 2'5. - Haldeman, Col. Longicorn. of the Unit. Stat. p. 65.271.

Habitat in peninsula Kenai rarius; a D. Holmberg ad castellum Nicolajevsk et a D. F. Frankenhaeuser ad rivulos fl. Tschunuktnu, exitu Augusti, in herbis inventa.

P. sexmaculatae Linné affinis, sed differt punctura crebriore et subtiliore, pubescentia breviore magis cinerea, thorace angustiore et elytris posterius magis angustatis et dehiscentibus, apice ipso non rotundato sed subtruncato, signaturis nigris aliter dispositis.

242. P A CHY TA F U L I P N Is : oblonga, nigra, nitida, tenue griseo-pubescens; thorace latitudine longiore, remote punctulato, basi apiceque constricto, lateribus modice rotundato, medio depresso, subtiliter canaliculato; elytris elongatis, sat crebre punctulatis, fulvis, intra humeros utrinque profunde impressis, postice nonnihil attenuatis, apice truncatis.

Longit. $3 \frac{1}{4}$ lin. Latit. 1 lin.

Ad rivulos fl. Tschunuktnu peninsulae Kenai, mense Augusto, in herbis a D. F. Frankenhaeuser rarissime capta.

P. marginata Fabr. varietati elytris fulvis valde similis, sed antennis longioribus, elytrisque longioribus, crebrius et subtilius punctulatis diversa.

243. Gram iортег a suarentata Kirby: elongata, nigra, capite thoraceque creberrime confertim punctulatis, hoc oblongo, antrorsum paullo angustato, in medio longitudinaliter subcarinato, basi bisinuato, angulis extrorsum prominulis acutis; elytris latitudine quadruplo longioribus, linearibus, profunde et crebre 


\section{0}

punctatis, apice rotundatis; supra parce griseo - pubescens, thoracis lateribus et angulis posticis corporeque subtus argenteo-sericeis.

Longit. $3 \frac{1}{2}$ lin. Latit. $\frac{2}{3}$ lin.

Leptura subargentata Kirby in Richardson, Fauna Bor Amer. IV. p. 184. 248. - Haldeman, Col. Longicorn of the Unit. Stat. p. 65.274.

Var. b. paullo brevior, antennarum articuli primi apice, femoribus quatuor anterioribus tibiisque anticis, tibiis intermediis introrsum et femoribus posticis apice excepto rufis. an Mas?

Longit. 3 lin. Latit. $\frac{2}{3}$ lin.

Leptura simless Kirby 1. c. p. 185. 249. - Haldeman I. e p. 65.275.

Iisdem locis eodemque tempore uti praecedens species a D. F. Frankenhaeuiser rarius lecta.

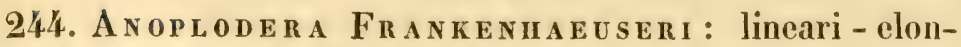
gata, nigra, griseo-pubescens; capite thoraceque creberrime rugulosis; hoc elytris dimidio angustiore, latitudine longiore, basi apiceque constricto, lateribus in medio utrinque in tuberculum validum dilatato, medio dorsi tenue canaliculato; elytris valde elongatis linearibus, subdepressis, profunde punctatis, apice subrotundatis, margine laterali lineaque longitudinali discoidali cum margine ad apicem suturae connexa flavo - testaceis; antennis corpore parum brevioribus, abdomine pedibusque ferrugineo-testaceis, illis articulis septem ultimis, his femoribus superne tibiisque extrorsum infuscatis. 


\section{1}

$V a q . b$. elytris flavo-testaceis, utroque vitta intramarginali, apicem haud attingente, nigra.

IIabitat ad rivulos fl. Tschunuktnu peninsulae Kenai, mensibus Julio el Augusto, in herbis rarius; D. F. Frantienhacuser.

Leptunae Proteo Kirby (Richardson, Fauna Bor. Amer. p. 186. 2ỏ1.) secundum descriptionem coloribus aflinis videtur, sed habitus speciei Kirbyanae hane Pacuyts adpropinqual.

245. An oplodera macilenta: lineari-elongata, nigra, griseo-pubescens; antennis corpore parum brevioribus; capite thoraceque creberrime rugulosis; illo in vertice canaliculato; hoc angustissimo, supra inaequali, basi apiceque constricto, lateribus utrinque in tuberculum validum dilatato; elytris valde elongatis linearibus, subdepressis, crebre et profunde punctatis, apice rotundatis; femorum basi geniculisque testaceis.

Longit. 4 lin. Latit. 1 lin.

Occurrit in eadem localitate ut praecedens ibique haud frequentius; D. F. Frankenhaeuser.

Statura elongata angusta praecedenti certe affinis et forsan ejus tantum varietas, sed ob thoracem multo angustiorem elytraque crebrius punctata ut speciem distinctam considerare malui.

\section{CHR Y SOMELINA.}

246. Bromus Vitis Fabricius: niger, cinereo-pubescens, crebre punctulatus; antennarum basi, tibiis elytrisque rufo - castaneis, fronte stria longitudinali exarata; elytris quodam aspectu obsolete striatis; pedibus elongatis, femoribus clavatis.

Longit. $2 \frac{1}{8}$ lin. Latit. $1 \frac{2}{5}$ lin. 
Dejean, Cat. 3-ème édit. p. 436 - Gebler, Bullet. de la Soc. de Moscou. 1848. II. p. 39. 2. - Redtenbacher, Fauna Austr. p. 558. - Gaubil, Cat. p. 197. 2.

Eumolpus Vitis Fabricius, Syst. El. I. p. 422. 20. - Panzer, Fauna Germ. XXXIX. 12. - Latreille, Gen. Crust. et Ins. III. p. 56. 2. - Schönherr, Syn. Ins. II. p. 236. 25. - Olivier, Entom. VI. 96. p 911. 28. Tab. I fig 9. Duftschmid, Fauna Austr. III. p. 216. 2. - Stephens, Ill. Mandib. VI. 363. Manual. p. 304 2387.-Cat. Col. Europ. 1852. p. 88.

Eumolpus (Adoxus) Vitis Kirby in Richardson, Fauna Bor. Amer. IV. p. 209. 279.

Cryptocephalus Vitis Fabricius, Spec. Ins. I. p. 142. 23. Ent. Syst. II. p. 60. 36.

Ad ostia fl. Kaktnu peninsulae Kenai, exitu Junii, a D. Holmberg semel inventus.

247. Chrisonela subulcata Motschulsty: subhemisphaerica, valde gibbosa, viridi-aenea, nitida; thorace transverso, apice parum emarginato, basi bisinuato, lateribus modice rotundato, antrorsum parum angustiore, subtiliter remote punctulato, margine late incrassato, sulco apicem usque profunde exarato utrinque limitato; elytris sulcatis, in sulcis punctis subtilibus remotis striatis, interstitiis valde elevatis, costatis, antice inaequalibus.

$V a r$. b. supra nigra, elytrorum margine viridiaeneo, corpore subtus obscure cyanescente.

Longit. $3 \frac{1}{4}-3 \frac{1}{2}$ lin. Latit. $2 \frac{1}{4}-2 \frac{1}{3} \operatorname{lin}$.

Specimina in insula St. Pauli a D. Wosnesensliy capta e Museo Acad. Imp. Scient. Petrop. ad describendum benevole transmisit $\mathrm{D}$. Ménétriés. 


\section{3}

248. Lina Lapponica Linné: breviter ovata, supra nigro - violacea, subtus nigra, nitida; antennarum articulis mediis rufo-cinctis; thorace transverso, antrorsum modice angustato, apice profunde emarginato, angulis anticis productis rotundatis, lateribus subrectis vel nonnihil sinuatis, disco laevi vel obsoletissime tantum punctulato, intra marginem utrinque oblique incrassato et in impressione profunde rugoso - punctato, margine laterali rufescenti - pellucido; elytris marginatis, profunde punctatis, pone medium apice simul sumtis rotundatis, utroque margine basali et laterali, in medio annulis duobus transversim positis, inter se connexis, externa cum margine laterali, interna cum limbo basali ad suturam decurrente contiguis, paullo pone basin macula parva et altera ante apicem oblongis, hac cum limbo marginali anguste cohaerente, rufo-testaceis.

Longit. 3 lin. Latit. $1 \frac{5}{4}$ lin.

Dejean, Cat. 3-ème édit. p. 426. - Gebler, Bullet. de la Soc. de Moscou. 1848. II. p. 31. 3. - Redtenbacher, Fauna Austr. p. 552. - Gaubil, Cat. p. 196. 6.

Cunysomela (Lina) Lapponica Cat. Col. Eur. 1852. p. 90.

Chrysomera Lapponica Linné, Fauna Suec. p. 165. 526. Syst. Nat. II. p. 591. 34. - Fabricius, Ent. Syst. I. 1. p. 321. 70. Syst. El. I. p. 437. 95. - Paykull, Fauna Suec. II. p. 57. 9. - Panzer, Fauna Germ. XXIII. 13. - Schönherr, Syn. Ins. II. p. 258. 103. - Gyllenhall, Ins. Suec. III. p. 463. 12. - Olivier, Entom. V. 89. p. 559 89. Tab. I. fig. 9. - Gebler, Mém. de la Soc. de Moscou. VI. p. 124. 9. in Ledebour Reise II. 2. p. 214. 20. - Zetterstedt, Fauna Lappon. I. p. 415. 6. Ins. Lappon. p. 227. 7. - Sahlberg, Ins. Fenn. II. p. 196. 8. - Suffian, Linnaea Ent. V. p. 196. 138. 


\section{4}

Merasoma Lapponica Stephens, IIl. Mandib. IV. p. 351.4. Manual. p. 307. 2421.

Ad fl. Tschuniten peninsulae Kenai, initio Junii, in salice tis a $\mathbf{D} . \boldsymbol{F}$. Frankenhaeuser rarius capta. Etiam in arena litorali ad ostia fl. Kaktnu a D. Holmberg inventa.

Quamvis statura breviore, thoracis lateribus rufescentibus elytrorumque signaturis in medio biannulatis et macula pone basin adjecta ab individuis nostris nonnihil discrepat, hujus speciei varietatibus numerosis forma et signaturis rufis admodum instabilibus tamen sit adnumeranda, co magis quam e Sibiria etiam possideo specimen, cujus maculae elytrorum in medio fere ita sunt connexae.

249. GONIOCTENA VIMINAL IS Linné: oblongo-ovata, convexa, nigra; thorace transverso, lateribus a basi ad apicem perparum rotundato-angustatis, apice late emarginato, angulis productis acutis, disco subtiliter remote, ad basin crebrius punctulato, versus latera profunde confertim punctato, rufo-testaceo, macula magna postica transversa nigra; elytris subtiliter punctato-striatis, interstitiis confertissime punctulatis; rufo - testaceis, maculis duabus pone basin, duabus in medio oblique positis, quarum prima, tertia et quarta cohaerentibus et quinta postice margini parallela oblonga, nigris; abdominis segmenti ultimi apice rufescente.

Longit. $2 \frac{3}{4}$ lin. Latit. $1 \frac{3}{4}$ lin.

Dejean, Cat. 3-ême édit. p. 427. - Gebler, Bullet. de la Soc. de Moscou. 1848. II. p. 3\%. 4. - Redtenbacher, Fauna Austr. p. 558. - Gaubil, Cat. p. 197. 6.

Chrsomela (Gonoctena) Viminalis, Cat. Col. Eur. 1852. p. 90 . 


\section{5}

Chrysomela vimnals Linné, Fauna Suec. p. 164. 524. Syst Nat. II p. 590. 31. - Fabricius, Ent. Syst. I. p. 319. 5?9. Syst. EI. I. p. 436. 83. - Payliull, Fauna Suec. II. p. 68. 21. - Schönherr, Syn. Ins. II. p. 254. 93. Gyllenhal, Ins. Suec. III p. 487. 30. Var. h. - Olivier, Entom. V. 89. p. 572. 108. Tab. IX. fig. 129 - Duftschmid, Fauna Austr. III. p. 200. 63. - Gebler in Ledebour Reise. II. 2. p. 217. 20.-Zetterstedt, Fauna Lappon. I. p. 420. 15. Ins. Lappon. p. 229. 17. Sahlberg, Ins. Fenn. Il. p. 210. 26-Suffrian, Linnaea Entom. V. p. 211. 150. Var. $\gamma$.

Chrosomela decempunctata Linné, Fauna Suec. p. 16 '. 525. Syst. Nat. II. p. 590. 32. - Fabricius, Ent. Syst. I. p. 320. 62. Syst. El. I. p. 436. 86. - Stephens, Ill. Mandib. IV. p. 338. 3. Manual. p. 311. 2450.

Chinsomela Baneri Panzer, Fauna Germ. XXIV. 14.

Specimen ad fl. Kiwichpalih captum e Mus. Acad. Imp. Scient. Petrop. communicavit D. Ménétriés.

250. Gonioctena A R Cica: oblongo-ovata, convexa, nigra; thorace transverso, lateribus modice rotundato, antrorsum attenuato, angulis anticis subacutis, dorso remote subtiliter, versus latera crebrius et profundius punctato, punctis majoribus intra angulos confluentibus, margine laterali, antrorsum latius, lineolaype media apicis rufo-ferrugineis; elytris punctato-striatis, punctis striarum pone callum humeralem et versus apicem hinc inde irregularibus, rufo-ferrugineis, singulo maculis quinque bifariam positis nigris, quarum duae interiores magnae oblongae, interdum cohaerentes, exteriores prima et terlia minores, intermedia vero majore subtransversa; antennis dimidia parle, margine segmenti ultimi abdominalis tibiisque extrorsum plus minusve rufo-testaceis. 


\section{6}

Gonoctena affinis Mannerh. Bullet. de la Soc. de Moscon. 1852. II. p. 369. 172.

$V a r . b$. nigra, antennarum basi, thorace antice lateribusque et elytris rufo-ferrugineis, utroque plagis duabus magnis oblongis nigris, externa cum medio internae contigua, punctoque pone medium versus marginem nigris, tibiis et ano ut in $a$.

Var. c. nigra, antennarum articulis inferioribus, tibiis, segmenti analis margine, elytrorumque limbo postico et apice rufo-ferrugineis.

Var. $d$. subtus nigra, thorace flavo-testaceo, punctis duobus postice transversim positis nigris, elytris rufo-ferrugineis, maculis duabus minutis mox pone basin etiam transversim positis nigris, antennis, pedibus segmentique analis margine rufo-ferrugineis, illis summo apice tarsisque nonnihil infuscatis.

Longit. $2 \frac{1}{3}-2 \frac{1}{2}$ lin. Latit. $1 \frac{5}{1} \frac{5}{2}-1 \frac{1}{\frac{1}{2}}$ lin.

In salicetis, medio Junii, ad fl. Tschunuktnu peninsulae Kenai sat frequens; D. F. Frankenhaeuser.

Ad G. affinem Schönh. jam retuli, sed individuis plurimis lustratis, de diversitate specifica convictus sum, quare diagnosis antea data ut et synonymia ibi allata supprimantur et pro his descriptio nunc accuratius claborata substituatur.

Statura el magnitudine G. Nivosaz Heer, Suffrian reapse magis quam afFinı Schönh. propinqua; thorace minus lato, lateribus magis rotundato antrorsum subito angustato angulisque anticis subacutis, ut et elytrorum striis pone callum humeralem et versus apicem hinc inde irregularibus ab illa; magnitudine vero minore, statura magis lineari, antennis multo longioribus thoracisque lateribus rotundatis ab hac distincta. 


\section{7}

251. Prratora interstitiais Ménétriés: oblonga, viridi - aenea, caerulescenti - micans, nitida; antennarum articulo primo apice rufescente; thorace longitudine duplo latiore, antice subito angustato, lateribus posterius subrectis, in medio remote, ceterum crebrius punctato, utrinque fovea magna obliqua (an fortuito?) impresso; elytris crebre punclatis, punctis versus suturam in strias seriatis.

Longit. 2 lin. Latit $\frac{3}{4}$ lin.

Puyllodecta Vitelunae? Kirby in Richardson, Fauna Bor. Amer. IV. p. 216. 289.

Habitat ad fl. Kwichpakh, sec. D. Ménétriés. Mus Acad. Imp. Scient. Petrop.

Phr. Vitellnae Linné statura affinis, sed elytrorum tota superficie profunde et crebre punctata sine ullo dubio species distincta.

252. Galceruca luctuosa Mannerheim: Bullet. de la Soc. de Moscou. 1852. p. 368. 171.

Var. b. ore thoracisque limbo anguste testaceis; elytris nigro-fuscis, margine laterali et apicali latius pallide testaceo; thorace ad canaliculam longitudinalem postice profunde impresso.

Longit. $2 \frac{5}{4}$ lin. Latit. $1 \frac{1}{2}$ lin.

Galleruca marginelea? Kirby in Richardson, Fauna Bor. Amer. IV. p. 220. 296.

Ad rivulum fl. Tschunuktnu peninsulae Kenai, initio Julii, excipulo e gramine a D. F. Frankenhaeuser semel capta.

253. Haltica tombacina: oblongo-ovata, convexa, fusco-cupreo-aenea, subnitida; thorace transverso, 


\section{8}

remote subtilissime punctato, lateribus vix rotundatis, apice truncato, angulis anticis dente minuto utrinque armatis, intra basin linea parum arcuata leviter impresso; elytris sat crebre subtiliter punctulatis, lateribus ante medium nonnihil sinuato - constrictis.

Longit. $2 \frac{1}{4}$ lin. Latit. $\frac{3}{4}$ lin.

Individum unicum ad rivulum fl. Tschunuktnu peninsulae Kenai, mense Augusto, in herbis invenit D. $F$. Frankenhacuser.

Statura fere II. ordinacene Linné, sed thorace antrorsum minus angustato, elytris remotius punctulatis et femoribus posticis magis incrassatis diversa.

254. Cassid a ов I is Linné: oblongo-ovata, convexa, griseo-flavescens; thorace brevi, lateribus et antice rotundato, basi trisinuato, limbo antico et laterali late ruguloso; elytris angulis hmmeralibus valde productis, disco profunde punctato - striatis, margine explanato, antrorsum sensim latiore, irregulariter punctato, subrugoso, interstitio secundo dorsali latiore, in vivis caerulescenti-argenteo, post mortem concolore; corpore subtus, femoribus basi et antennarum apice plus minusve nigris.

Longit. $2 \frac{1}{3}$ lin. Latit. $1 \frac{7}{1} \mathrm{~d}$ lin.

Linné, Famna Suec. p. 153 469. Syst. nat. II. p. 575. 4. Fabricius, Ent. Syst. I. p. 297. 24. Syst. El.I p. 396.47.Illiger, Käfer Preus. I p. 485. 11. - Payliull, Fauna Suec. II. p. 49. 97. - Herbst; Col. VIII. p. 23816. Tab. 130. fig. 15. - Olivier, Entom. V. 97. p. 983.104. Talb. II. fig. 24. - Schünherr, Syn. Ins. II. p. $219.68-$ Gyllenhal, Ins. Suec. III. p. 4h5. 9. - Stephens, III. Mandil. IV. p. 370. 15. Manual p. 287. 22/6. - Zellerstedt, Faum. Lappon. I. p. 101. 5. Ins. Lappon. p. 220 5.- 
Saltberg, Ins. Fenn. 11. p. 190. 9. - Dejeun, Catl. 3-ime édit. p. 399. - Suffrian, Stett. Entom. Zeit. 184h. p. 2'5. 19. - Gebler, Bullet. de la Sor. de Mosen. $18: 8$. II. p. 9. 6. - Redtenbacher, Fauna Austr. p. 521. Gaubil, Cat p. 188. 29. - Catt. Col. Eur. 1852. p. 9:3.

Specimen ut in insula Sitlha captum en Hoseo Acad. Imp. Scient. Petrop. transmisit i). Ménétriés. Anne eum rebus hortensibus Europeis in statu larvac vel pupae importatum?

\section{O C C I N E L LIA.}

255. HIPPO D M A TREDECIMPUNCT A T Limbé oblonga, subtus nigra; capite niogro, ore luteo, macula margini apicali contigua frontali, subhastata albida; thorace nigro, margine antico anguste, laterali vero late lobum rotundatum disci nigrum utrinque includente, flavo-albidis; elytris fulvis, maculis nigris in utrofue sex, 1. 2. 2. 1. scutellarique communi oblongiuscula; tibiarum apice tarsisque ferrugineis.

Var. b. thoracis nigredine magis dilatata, antice profunde bisinuata, elytrorum macula secunda cum suturali utrinque connexa.

Longit. $2 \frac{1}{2}-2 \frac{3}{4}$ lin. Latit. $1 \frac{1}{2}-1 \frac{2}{3}$ lin.

Dejean, Cat 3-ême édit. p. hä6. - Stephens, 111. Mandib.

VI. p. 388. Manual p. 317. 2198. - Mulsent, Sécuripalpes de France p. 31. 1. Spec. des Col. Trim. Sécurip. p. 10. 1. - Gebler, Bullet de la Soc. de Moseon. 18's.

II. p. 58. 3. - Gaubil, Cat. p. 205. 1. - Cat. Col. Eur. 1852. p. 93.

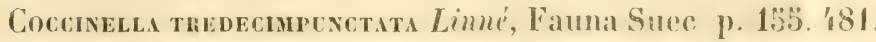

Syst. Nat. II. p. 582. 20.-Fabricius, Ent. Syst 1. I. p. 279. 61. Syst. El. I. p. 369.76. - Ilerbst, Col. V p. 323. 79. Tab. 57. Gig. 11. - Sthncider, Mag. 139. 8- 
Panzer, Fauna Germ. CXII. 8. - Paykull, Fauna Suec. II. p. 42. 44. - Schönherr, Syn. Ins. II. p. 174. 58. Olivier, Entom. VI. p. 1023. 'Tab. VI. fig 79. - Gyllenhal, Ins. Suec. IV. p 207. 46. - Kirby in Richardson, Fauna Bor. Amer. IV. p. 229. 310. - Redtenbacher in Germar Zeischrift. V. p. 126. Fauna Austr. p. 581.

Ad ostia fl. Kaktnu peninsulae Kenai, exitu Junii, in herbis a D. Holmberg frequenter capta.

Media quasi inter H. tredecrmpunctatam et septemmaculaтам Linne; colore nempe capitis, thoracis et pedum huic adpropinquat, sed forma totius corporis ab illa haud sejungi licet.

256. Adonia PARENTresis Melsheimer: ovalis, capite maculis tribus albidis, media lineari ab ore ad verticem protensa; thorace margine antico et laterali anguste, apice interrupto maculaque basali quadrata flavo-albidis; elytris flavo-testaceis, maculis nigris: scutellari communi oblonga postice dilatata, humerali magna, prope illam extus posterius minutissima, magna obliqua in disco trapezoidali cum extrorsum arcuata postica angulo externo connivente: corpore subtus nigro, epimeris albis, antennis, tibiarum apice tarsisque rufescentibus.

Var. b. thoracis margine antico albido, lineolam in discum emittente, macula basali rotundata; elytris macula arcuata postica lobo ad suturam dilatato, postice versus apicem acuminato-protenso.

Longit. $1 \frac{3}{4}-2$ lin. Latit. $1 \frac{1}{4}-1 \frac{1}{3}$ lin.

Mulsant, Spec. des Col. Trim. Sécurip. p. 40. 3

Hrppodama fastidita Dejean, Cat 3-ême édit. p. 456.

Hippodama curvata Chevrolat in litteris. 


\section{1}

Hippodama Lumatomaculata Motschulsky, Bullet. de la Soc. de Moscou. 1845. II. p. 382. 78. Tab. VII. fig. 8. 8.

Coccinella panentuesis Malsheimer, Cat. Say in Journ. of the Acad. of Philadelph. IV. p. 93. 3.

Coccinelua tridens Kirby in Richardson, Fauna Bor. Amer. IV. p. 229. 311.

Coccinela septemlonata Eschscholtz in litteris.

Iabitat ad rivulos fl. Tschunuktnu peninsulae Kenai; D. F. Frankenhaeuser. Medio Septembris in volatu rarissime obvia.

257. HARMONIA DUODECIMMACULTA Gebler: subhemisphaerica; capite nigro, antennis, ore margineque laterali et apicali rufis; thorace incarnato-rufo, maculis duabus magnis, transverse subquadratis, discum fere totum occupantibus, nigris; elytris incarnatis, maculis tribus communibus suturalibus, quarum prima scutellari ovata, secunda pone medium rotundata tertiaque ante-apicali minuta subtransversa, et praeterea in utroque quatuor magnis, quarum prima humerali obliqua, secunda et tertia in medio transversim positis subrotundatis quartaque juxta secundam suturalem collocata, nigris; corpore subtus nigro, abdominis lateribus et ano, coxis, femorum tibiarumque apice et tarsis rufis.

Longit. $2 \frac{2}{3}$ lin. Latit. $2 \frac{1}{4}$ lin.

Mulsant, Spec. des Col. Trim. Sécurip. p. 86. 8.

Coccinella nuodecmmacelata Gebler, Mém. de la Soc. de Moscou. VIII. p. 76. 3.

Coccinella incarnata Kirby in Richardson, Fauna Bor. Amer.

IV. p. 231. 315. Tab. VII. fig. 7.

Coccinela Daunca Dejean, Cat. 3-ème édit. p. 4.57.

Coccinelua duodexari. Eschscholli in litteris. 
Ad fl. Tschuniten peninsulae Kenai, initio Junii, in volatu a D. F. Frankenhaeuser semel inventa.

258. Coccinella trifasciata Limné: subhemisphaerica; capite maris flavo, fascia postica nigra, feminae nigro, maculis duabus frontalibus transversim positis flavis: thorace nigro, angulis anticis utrinque macula obliqua flavo - albida, introrsum sinuata notatis, et in mare margine antico etiam flavo; elytris fulvis, regione juxta-scutellari flavo, fasciis tribus transversis, latera non attingentibus, quarum tantum antica ad suturam continuata ibique cum opposita et scutello contigua, nigris; corpore subtus nigro, epimeris albis, abdominis segmento primo utrinque macula flava.

Longit. $2 \frac{1}{9}$ lin. Latit. 2 lin.

Linné, Fauna Suec. p. 154. 475. Syst. Nat. I. p. 580. 13. Fabricius, Ent. Syst. I. 1. p. 95. 14. Syst. El. I. p. 363. 42. - llerbst, Col. V. p. 330. 81. Tab. LVII. fig. 13. Paykull, Fauna Suec. II. p. 17. 17. - Schönherr, Syn. Ins. II. p. 170. 52. - Olivier, Entom. VI. p. 1003. 24. Tab. III. fig. 37.- Gyllenhal, Ins Suec. IV. p. 166. 18. Zetterstedt, Fauna Lappon. I p. 432. 7. Ins. Lappon. p. 235. 8. - Stephens, Ill. Mandib. IV. p. 381. Manual. p. 315. 2186. - Gebler, Bullet. de la Soc. de Moscou. 1848. II. p. 61. 21. - Mulsant, Spec. des Col. Trim. Sécurip. p. 119. 27. - Gaubil, Cat. p. 207. 10. - Cat. Col. Eur. 1852. p. 94.

IIabitat ad ostia fl. Kaktnu peninsulae Kenai, ubi exitu Junii, recessu maris, in arena litorali a D. Holmberg sacpius'lecta.

\section{T E N E B R I O N I A.}

DY S MATHES.

Antennae capite cum thorace breviores, articulis coarctatis, subcylindricis; primo brevi, crassiore; secundo sesqui 
breviore; tertio hoc fere duplo longiore; $4-10$ sensim parum brevioribus; ultimo praecedente vix longiore, apice obtuse rotundato.

Labrum transversum, apice profunde emarginatum.

Palpi omnes articulis subcylindricis, ultimo apice parum angustato ibique subrotundato.

Caput deplanatum, antice subquadratum, pone oculos nonnihil dilatatum, thorace angustius; oculi minuti, subreniformes.

Thorax deplanatus, transversus, antrorsum angustatus, apice late emarginatus, capiti arcte applicatus, basi bisinuatus, angulis posticis acutis, extrorsum prominulis, medio longitudinaliter canaliculatus, lateribus in medio rotundato - dilatatis, serratis.

Elytra connata, modice convexa, thorace duplo latiora et plus quam quadruplo longiora, elliptica, humeris rotundatis.

Pedes tenuiores: tibiae quatuor posteriores extrorsum nonniliil arcuatae, omnes apice brevissime bispinosae; tarsi quatuor anteriores quinque -, postici quadri - articulati, articulo ultimo reliquis multo longiore, nonnihil incurvo, unguiculis aequalibus parum curvatis, ceteris articulis subcylindricis compressis, subtus glabris.

Genus admodum paradoxum, statura Nxcrebirs quodam modo simile, sed antennarum structura genera PACHYcinlam et Grathosiam Tentyrionum in memoriam revocat, ideoque transitum inter has tribus constituere videtur.

259. Dysmaties Sandiergil：atra，opaca; capite crebre punctato, fronte juxta oculos utrinque longitudi- 


\section{4}

naliter impressa; thorace profunde rugoso - punctato, intra angulos anticos et posticos impresso; elytris striatis, rugoso-punctatis; antennarum articulo ultimo rufescente; corpore subtus pedibusque nigro-piceis.

Longit. $5 \frac{3}{4}$ lin. Latit. $2 \frac{3}{4}$ lin.

Individuum unicum in insula Sitkha, sub muscis in lapide crescentibus, invenit D. Ferd. Sahlberg.

\section{SE R R O P A L P I A.}

260. Serropalpus striatus Hellenius: elongatus, convexus, fusco-brunneus, subtilissime transversim ruguloso-punctatus; thorace longitudine vix latiore, lateribus rotundato, antice parum angustiore, margine laterali in aciem carinato, angulis posticis subacutis, ante basin utrinque oblique leviter impresso; elytris singulatim subacuminatis, leviter striatis, subsulcatis; corpore subtus cum pedibus dilutius ferrugineocastaneo.

Longit. $4 \frac{1}{3}$ lin. Latit. 1 lin. (Occurrunt apud nos individua plus quam duplo majora.)

Hellenius, Act. Holm. 1786. p. 318. 1. Tab. 8. fig. 3-5.Latreille, Hist. nat. des Crust. et des Ins. X. p. 338. Gen. Crust. et Ins. II. p. 193. 1. Tab. IX. fig. 12. - Paykull, Fauna Suec. II. p. 163. 1. - Illiger, Käfer Preus. I. p. 130. 7. - Gyllenhal, Ins. Suec. II. p. 515. 1. - Schönherr, Syn. Ins. III. p. 47. 1. - Sahlberg, Ins. Fenn. I. p. 447. 1. - Redtenbacher, Fauna Austr. p. 609. - Gaubil, Cat. p. 227. 1.

Serropalpus barbatus Zetterstedt, Fauna Lappon. I. p. 283. 1. Ins. Lappon. p 163. 1. - Dejean, Cat. 3-ème édit. p. 223. - Gebler, Bullet. de la Soc. de Moscou. 1847. II. p. 483. 1. - Cat. Col. Eur. 1852. p. 62. 


\section{5}

Dircaea marmata Fabricius, Ent. Syst. Suppl. p. 121. 1. Syst. El. II. p. 88. 1. - Duftschmid, Fauna Austr. II. p. 264. 1 .

Lymexylon barbatun Olivier, Entom. II. 25. p. 5. 3. Tab. I. fig. 3.

Ad rivulos fl. Tschunuktnu peninsulae Kenai, exitu Augusti, in volatu a D. F. Frankentaeuser rarius captus.

261. HALL MENUS BASALIS: elongatus, antice posticeque angustatus, rufo-testaceus, subtilissime rugoso-punctulatus, griseo-pubescens; capite nigro; thorace nigro-fusco, lateribus lineaque media longitudinali rufo-testaceis, basi utrinque foveola leviter impressa; elytris ante medium posterius infuscatis.

Longit. $2 \frac{1}{4}$ lin. Latit. $\frac{2}{3}$ lin.

Exemplare singulum, volitans ad unum ex rivulis fl. Tschunuktnu in peninsula Kenai, cepit D. F. Frankenhaeuser.

H. uumenati Illig. Gyllenh. nonnihil brevior, aliter coloratus, thoracis foveolis basalibus minoribus et striis elytrorum haud conspicuis diversus.

\section{O E D E M E R I A.}

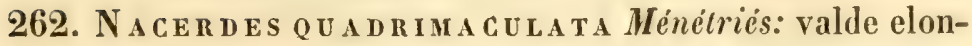
gata, angusta, creberrime subtiliter punctulata, dense cinereo-pubescens, pallide flavo-testacea, vertice, oculis, pectore, abdomine (segmento ultimo excepto) genubusque nigro-fuscis; thorace latitudine plus duplo longiore, e medio posterius abrupte coarctato, basi apiceque marginato, plicato, angulis posticis prominulis, maculis tribus ante medium transversim dispositis et quarta ad medium basis nigro-fuscis; elytris infuscatis, utroque lineis tribus angustis longi- 
tudinalibus, parum elevatis, apice singulatin rotundatis.

Longit. $6-6 \frac{1}{2}$ lin. Latit. $1 \frac{1}{4}-1 \frac{1}{3}$ lin.

Probosca quadraculata Motschulsky, Eludes entom. 1852. p. 78.

Specimen in insula Sitkha captum communicavit D. Minétriés. Species Californiae borealis reapse incola verisimile facit individuum hoc indidem in insulam Sitkham navibus introductum fuisse.

\section{P Y R O C H R T A.}

263. Pутіо deplanatus: elongatus, depressus, glaber, nigro-piceus, nitidus, ore, antennis, thoracis margine apicali et macula utrinque oblonga laterali antica, elytris, abdominis margine et apice, tibiis tarsisque rufo-testaceis; capite thoraceque profunde remote punctatis; vertice canaliculato; thorace angustiore subcordato, medio canaliculato, disco utrinque profunde et late arcuatim impresso, lateribus incrassatoelevato; elytris profunde sulcatis, sulcis subcrenatis, interstitiis elevatis angustis laevibus, superficie versus basin laevigata, remote tantum punctulata.

Longit. $6 \frac{2}{3}$ lin. Latit. $2 \frac{1}{3}$ lin.

Individuum e vicinitate fl. Kiwichpakh, in Musen Acarl. Imp. Scient. Petropol. asservatum, transmisit D. Mínétriés.

P. Depresso Linné valde affinis, sed ab illo tamen discrepat capite thoraceque multo remotius punctatis, vertice canaliculato, thorace angustiore, lateribus multo minus dilatato et antice magis incrassato, ut et elytris latioribus, nonnihil brevioribus, minus deplanatis, profundius sulcatis et basi remotius punctulatis. 


\section{9}

An Pytho Amencases Kirby (Richardson Fauma Bor. Amer. IV. p. 165. 219.) a cujus varietate $c$. thorace lateribus rufis pectoreque toto nigro diversus?

264. Pogonocerus EPHemeroides Ménétriés: Mannerh. Bullet. de la Soc. de Moscou. 1852. 1. p. 348. 130. (Femina.)

Mas: angustior; oculis in fronte conniventibus; antennis dimidio corporis fere longioribus (femina multo breviores.), articulis $3-10$. appendicem longissimam filiformem cuique emiltentibus, ultimo praccedente quadruplo longiore etiam filiformi; thorace angustiore, antrorsum attenuato, stipitato, in medio basis foveolato; elytris vix conspicue costatis.

Longit. 6 lin. Latit. 2 lin.

In truncis putrefactis domi vetustae villac Orlovskaja Odinolschka ad sinum Igatsch insulae Kadjak specimina aliquot, medio Julii, legit D. Holmberg.

\section{A N T H I C I A.}

265. AnTuICUS NigRta: niger, parum nitidus, cinereopubescens; capite quadrato, vertice carinato, thoraceque confertim subtiliter rugoso - punctatis; hoc latitudine vix longiore antrorsum lateribus valde rotundatodilatato ibique margine explanato; elytris oblongis, creberrime punctulatis, in medio nonnihil dilatatis.

Longit. $1 \frac{2}{5}$ lin. Latit. $\frac{1}{2}$ lin.

IIabitat in arena littorali, exitu Junii, ad ostia fl. Kaktnu peninsulae Kenai, non infrequens; D. Holmberg.

A. atro Panz. Gyllenh. Laferté magnitudine aequalis, sed minus elongatus, latior, cinereo-pubescens, vertice carinato, thorace breviore, antrorsum multo magis dilatato, margine explanato, ely tris medio dilatatis et puncturat subtili creberrima ab illo practerea dignoscendus. 


\section{Käfer - Irrten,}

die in anderen Gegenden der Nord-Amerikanischen Länder des Russischen Reiches gefunden worden sind, als in den bei den Beschreibungen bereits angegebenen.

Notropurus syzraticus Eschsch. - Kenai (ad fl. Tschuniten, initio Junii, sub lapidibus rarius).

Nebnia Metaluca Eschsch. - Kenai. - Afognak, - Kadjak (sub lapidibus et lignis, per totam aestatem, ubique frequens). - Atkha.

- gregaria Eschsch. - Atkha.

- Saulbergir Eschsch. Vä. b. Mannerh.. - Kadjak.

Leistus fernugivosus Mannerh. - Kenai.

Crcurus angusticolus Eschsch. - Kenai.

- marginatus Eschsch. - Kenai. - Afognak. - Kadjak (sub lapidibus et in ligno putrido sat frequens.).

Canabus Chamissonis Eschsch. - Kadjak (ora occidentalis; in quisquiliis domesticis Aleutorum, mense Julio, rarius.).

- Baccrvonus Eschsch. - Afognak. - Kadjak (locis aridis sub lapidibus, per totam aestatem, ubique vulgaris.).

Stenolopuus nigrines Eschsch. - Kadjak (sub lapidibus sat frequens.).

Hypuenpes yalides Eschsch. - Afognak. - Kadjak (mense Julio, minus frequens.). - Atkha.

Botunioptenus anstictus Eschsch. - Atkha.

Cryodius pinguedineos Eschsch. - Afognak (mense Augusto, sub lapidibus frequens.). - Kadjak.

- mparius Eschsch. - Chtagaluk. - Kenai (sub lapidibus et cortice arboreo sat frequens; ad sinum Woskresensk, mensibus Majo et Junio; ad rivulos fl. Tschuktnu, initio Julii.). - Kadjak (non infrequens, initio Junii.). 
Leinus mbanogastricus Eschsch. - Kadjak (sub lapidibus frequens.).

Cema nemotestriata Eschsch. - Kenai (ad ostia fl. Kaktnu, exitu Junii, in litore maris sub lapidibus et lignis sat frequens.). - Kadjak (in ora orientali, per totam aestatem, etiam sub lapidibus frequens.).

Calatuus ingratus Eschsch. - Afognak (mense Angusto, sub lapidibus sat frequens.). - Kadjak (mensibus Julio et Augusto, sub lapidibus ubique vulgaris.).

Patnones fossipnoss Eschsch. - Chtagaluk (initio Junii, sub lapidibus frequens.). - Afognak (mense Augusto, sub lapidibus rarius.). - Kadjak (etiam sub lapidibus ubique frequens.).

Trecuus oripennis Motsch. - Chtagaluk (initio Junii, sub lapidibus locis humidis minus frequens.).

- Califonnicus Motsch. - Chtagaluk (iisdem locis eodemque tempore quoque minus frequens.). - Kenai (ad sinum Woskresensk, medio Maji, sub lapidibus rarius.). - Afognak. - Kadjak (etiam sub lapidibus sat frequens.).

Perrpuns incertus Motsch. - Kenai (ad sinum Woskresensk, medio Junii, sub lapidibus rarius.).

- planusculus Mannerh. - Kenai (ad ostia fl. Kaktnu, exitu Junii, in litore maris sub lapidibus rarius.).

- Quadnifoveoutus Mannerh. - Kenai (Ad fl. Skeljanktnu, mense Septembri, sub lignis non infrequens.).

- rontisrratus Motsch. - Kenai (ad ostia fl. Kaktnu, exitu Junii, in litore maris sub lapidibus rarius; etiam in regionibus interioribus ejusdem peninsulae.).

Cocrunetes divisus Eschsch. - Kenai (in stagnis; ad sinum Woskresensk, exitu Maji, frequens; ad castellum Nicolajersk, exitu Junii, rarius.)

Irybus quadruaculatus Aubé. - Kadjak (mense Julio, in stagnis minus frequens.). - Atkha.

Agabus tristis Aubé. - Sitkha. - Kenai. - Kadjak (in stagnis per totam aestatem frequens.). - Atkha.

- vunius Mannerh. - Kenai (ad sinum Woskresensk, exitu Maji, in stagnis frequens.). - Kadjak. - Unalaschka. 


\section{0}

Agabus iyponelas Mannerh. - Edgecombe.

- scapulanis Mannerh. - Kadjak (in stagnis rarius.).

- antirnacinus Mannerh. - Kenai.

Hydroporus griseostriatus De Geer. - Kadjak.

Cercyon fimbratum Mannerh. - Kadjak.

- adumbatum Mannerh. - Chtagaluk.

Homalota granulata Mannerh。

Pricifica (Aleociura) AIotsch. - Chtagaluk. - Kadjak.

Arrocuara suleicolus Iannerh. - Afognak. - Kadjak (sub fucis e mare rejectis frequens.)

Tacindes circumcixctus Mäklin. - Kenai.

Otuus macrocephalus Eschsch. - Chtagaluk. - Kenai.

Staphyunus Bicinctus Eschsch. - Kenai (ad castellum Nicolajevsk, exilu

Junii, in quisquiliis et cadaveribus frequens.). - líadjak. -

Unalaschka. - Atkha.

- Cassus Mannerh. - Karjak (mense Augusto, in arena litorali sub

fucis frequens.). - Ins. St. Georgii.

Puicontues Siegwaldi Mannerh. - Chtagaluk. - Kenai. - Afognak. Kadjak.

- Canescens Mäklin. - Afognak.

Quedius Longrpennis Mannerh. - Kenai.

- pediculus Eschsch. - Kadjak.

- molocuinus Grav. - Chtagaluk. - Kenai. - Atkha.

Oxvtelus Fuscipenvis Mannerh. - Clatagaluk.

Epuraea truxcatele Mannerh. - Chtagaluk. - Kadjak.

Ips DeJeanı Kirby. - Kienai (ad sinum Woskresensk, initio Junii, sul cortice arboreo vulgaris.) - Kadjak.

Rinzophagus onmidus Eschsch. - Chtagaluk. - Kenai (ad sinum Woskresensk, medio Maji, sub cortice arboreo rarius.). - Afognak.

Atomaria fulvipensis Mannerh. - Chtagaluk. - Kenai. - Kadjak.

Latiridius protexsicollis Mannerh. - Kadjak.

- sobrives Mannerh. - Kadjak.

Melanophila appendiculati Fabr. - Cont. Amer. bor. ocćid. (all fl. Kivichpakh.) 


\section{1}

Enplaxis convutes Eschsch. - Kadjak (specimina plura in fenestra domi ad portum Pauli, mense Julio, legit D. Holmberg.).

A thous Fenncinosus Eschsch. - Kaljak (meusibus Julio et Augusto, suh cortice pini minus frequens.). - Atkha.

Crrptonypus musculus Eschsch. - Kenai (ad sinum Woskresensk, exitu Maji, sub lapidibus et lignis rarius.). - Kadjak.

Pristilopius angustrcolus Mannerh. - Chtagaluk. - Kadjak (mense Augusto, sub cortice pini rarius.). - Atkha.

Rhagonycia binodula Mannerh. - Kenai. - Kadjak.

Ptinus fur Linné. - Kadjak.

Cis Brarutus Mannerh. - Kenai (ad fl. Tschunuktnu, mensibus Junio et Julio sat frequens.).

- Anericaves Motsch. - Kenai (ad fl. Tschunuktnu codem tempore non infrequens et ad sinum Woskresensk.)

Bostricuus ramens Ménétr.-Cont. Amer. bor. occid. (ad fl. Kiwichpakh.)

Var. b. Mannerh. - Kenai (exitu Junii ad castellum Nicolajevsk.).

- Nitrdulus Mannerh. - Kenai (ad sinum Woskresensk, exitu Maji. sub cortice arboreo rarius.). - Kadjak.

Hycastes pumiLus Mannerh. - Chtagaluk.

Plintuus carinatus Eschsch. - Chtagaluk. - Kadjak.

Pytuo Sallbengil Mannerh. - Kenai. 



\section{E R R A T A.}

pag. 16 lin. 23 ausmachen,

- 23 - 12 afscende pa

- 30 - 3 litera

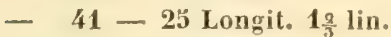

- 47 - 3 rejectes

- 54 - 27 rufo-discrepans.

- $57-16$ ride-aeneus,

- $\quad$ - 17 bifulcato;

- $62-26$ foreolatis

- 69 - 26 rarum

- $76-1$ testellatus

- $\quad$ - 7 testellatum

- $\quad-22$ insulae

- 77 - 31 citato

- $79-18$ dilata.

- 81 - 20 meum

- 83 post lin. 5 adde:

- 89 lin. 13 angulisque,

- $93-27$ (《Maklin.»)

- $94-12$ rotundato-amplicatis

- 93 - 15 incrassatis;

- 103 - 29 Latit. $\frac{3}{8}$ lin.

- $105-14$ species

- 108 - 26 Bollani

- 109 - 5 Bollan.

- 111 - 11 Latit. $\frac{5}{6}-$ lin.

- $\quad$ - $13 \mathrm{BLAMBUS}$

- 112 - 21 Bollan;

- 113 - 29 Máklin.

- -30 utroque

- $114-14$ fusco nigris.

- -27 invinit lege: ausmachen, bekannt zu thun,

- afseende på

- litora

- Longit. 2\% lin.

- rejectis

- rufo discrepans.

- ridi-aeneus,

- bisulcato;

- haud foveolatis

- ramum

- Tessellatus

- Tessellatui

- insula

- citata

- dilatata.

- mecum

Elytra elliptica, tenue marginata. lege: angulisque

- (《Mäklin.»)

- rotundato-ampliatis

- incrassatis,

- Latit. $\frac{5}{6}$ lin.

- speciei

- Coltani

- Collan.

- Latit. $\frac{5}{6}-1$ lin.

- Ciambes

- Collan:

- Mäklin.)

- utrisque

- fusco-nigris.

- invenit 
pag. 137 lin. 10 acute:

$-143-18$ si

- $144-19$ conniventibus;

- 175 - 3 nigris apicem

- 1 öă - 18 canalicuto,

- 159 - 11 signaturis

- 161 post lin. 2 adde:

- 162 lin. 17 Chrisomera lege: acute

- si-

- conniventibus,

- apicem

- canaliculato,

- signaturisque

Longit. 41 lin. Latit. $1 \frac{1}{6}$ lin.

lege: CH RY SOMLA

Indem dieser dritte Nachtrag zu meiner Käferfauna der Nord-Amerikanischen Länder des Russischen Reiches bereits gedruckt war, bekam ich zur Ansicht die Annalen des Naturhistorischen Lyceums zu New York (Annals of the Lyceum of Natural IIistory of New York.) für September 1831 und Februar 1832, eine Abhandlung enthaltend unter dem Titel: Descriptions of New Species of Coleoptera from California. By John L. Leconte. Es findet sich darin pag. 213 und 216 die Beschreibung eines neuen Käfers zur Staphylinen-Familie gehörend und Tinopinus pictus benannt. Ich erkenne in demselben den hier in diesem Nachtrage charakterisirten Trichocantuus Variegatus Motschulsky, weswegen dieser Name jenem älteren also weichen muss. 




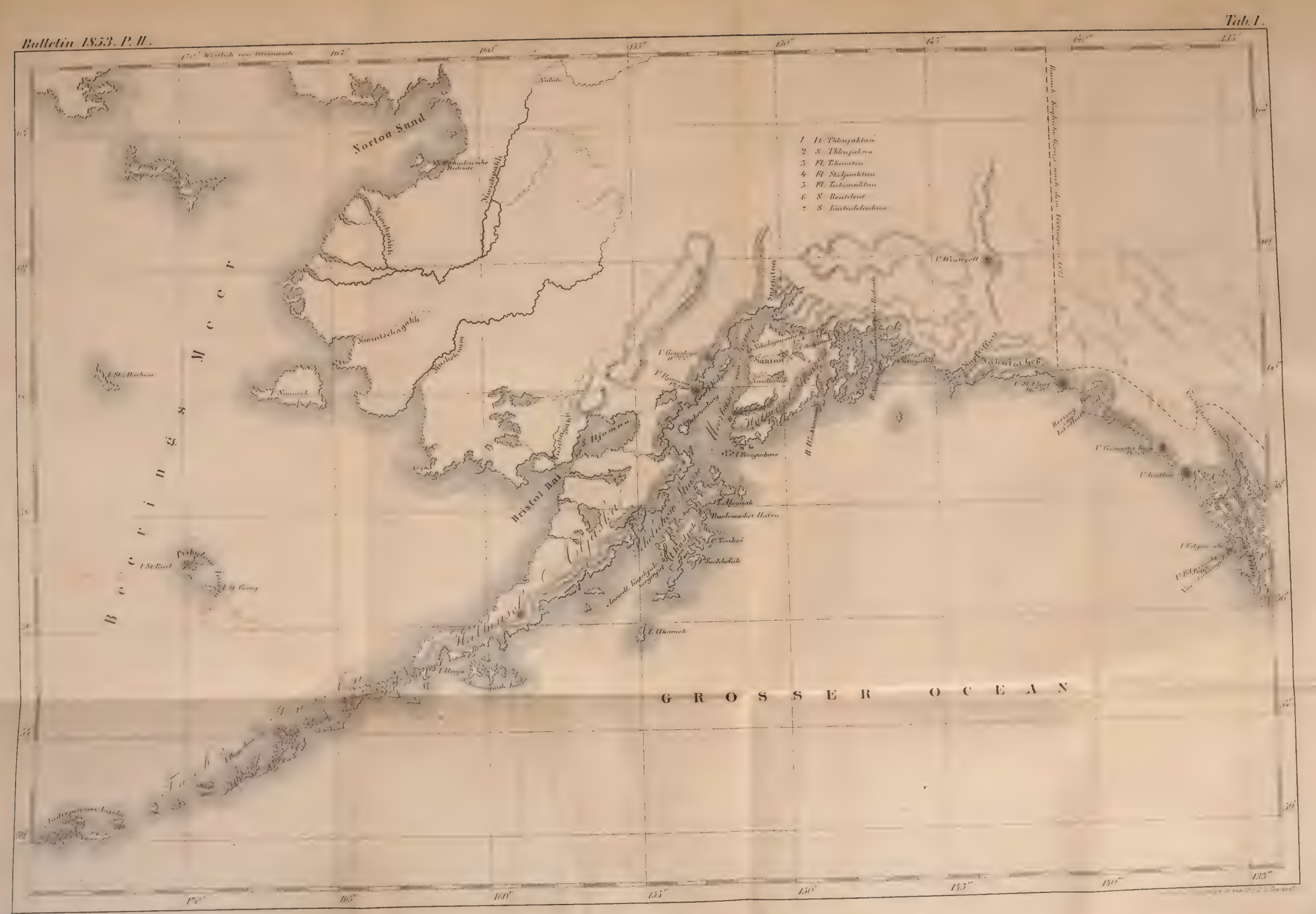













